\title{
Modeling of Residual Stress Fields and their Effects on Fatigue Crack Growth
}

\author{
by \\ Christian Jesús García López, B. Eng \& M. Sc.
}

A thesis submitted to the Faculty of Graduate and Postdoctoral Affairs in partial fulfillment of the requirements for the degree of

Philosophical Doctorate

in

Aerospace Engineering

Carleton University

Ottawa, Ontario

C 2015, Christian Jesús García López 


\section{Abstract}

Fatigue Crack Growth (FCG) is a deleterious physical phenomenon in engineering materials, which is intensified by the presence of tensile Residual Stress Fields (RSF), while compressive RSF has been shown to delay the FCG phenomenon. However, several challenges make it difficult to fully incorporate the beneficial effects of compressive RSF into the design process in aerospace and other engineering industries. As such, this study is designed to understand and quantify the effects of RSF on the FCG phenomenon in thick aluminum alloy specimens.

Experimental studies were conducted on specimens made of 7050-T7451 aluminum alloy in order to obtain the material properties required for a FCG model. In addition, FCG tests on Single-Edge Notched Tension (SENT) specimens with well-defined RSF were conducted for the verification of the FCG model.

Finite Element Analysis (FEA) software (ABAQUSTM) was used to simulate the FCG in RSF, and to analyze the redistribution of RSF due to FCG. ABAQUS was used first to introduce a RSF through a well-controlled four-point bending simulation, which was set as an initial condition to the FCG simulation. Several FCG test simulations were conducted to evaluate the crack closure and plastic wake effects on FCG. As part of these simulations, three test cases were considered: a large stress ratio $(R=0.7)$, a low stress ratio $(R=0.05)$ and a negative stress ratio $(R=-1)$.

For test case $1(R=0.7)$, the calculation of the FCG rate shows no indication of crack growth retardation due to the presence of the compressive RSF. However, the FCG rate was retarded in the test cases with low and negative stress ratios $(R=0.05$ and -1$)$. The 
plastic deformation induced at the crack tip due to FCG decreased under a positive effect of compressive RSF. In addition, the crack closure and plastic wake effects had a greater influence in the test case with a low stress ratio $(R=0.05)$, while their possible effects were not significant at $R=0.7$ and -1 , as demonstrated by the experimentally verified numerical model presented in this manuscript. 


\section{Acknowledgements}

I would like to express my recognition and gratitude to my supervisors Professors Andrei Artemev and Marcias Martinez for their invaluable support and guidance in the last four years to accomplish my research work.

Also, I want to thank to Carleton University and the Mechanical and Aerospace Department for welcoming me as one more of their students. Many thanks to all my Professors and the staff of the University.

I would also like to express my gratitude to the Consejo Nacional de Ciencia y Tecnología (CONACyT) and Roberto Rocca Education Program (RREP) for granting me a scholarship to pursue my Ph.D. studies. 


\section{Table of Contents}

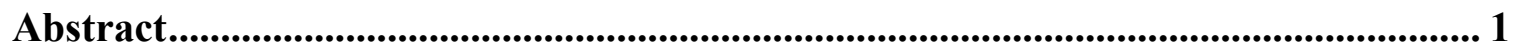

Acknowledgements ........................................................................................................................... 3

Table of Contents .................................................................................................................................. 4

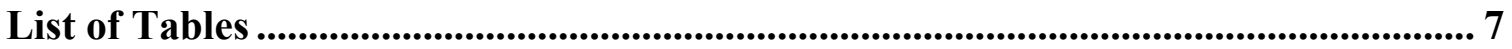

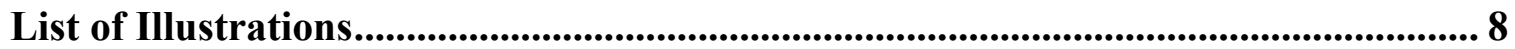

1 Chapter: Introduction ....................................................................................................... 18

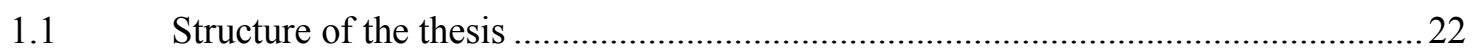

2 Chapter: Background and literature review............................................................. 25

2.1 The physics of the fatigue crack growth phenomena ..........................................25

2.2 Design philosophies for the avoidance of fatigue failures...................................27

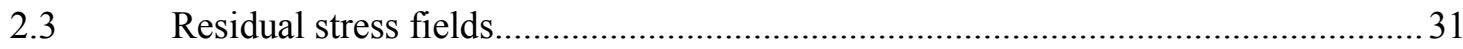

2.4 Experimental methods for the study of the fatigue crack growth in residual stress

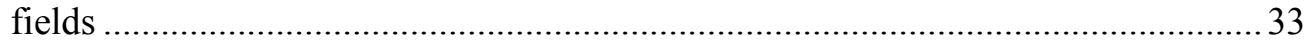

2.5 Numerical models for fatigue crack growth in residual stress fields ...................... 45

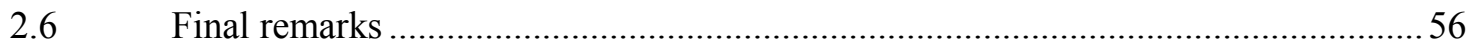

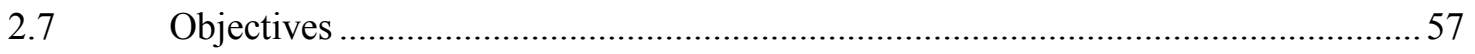

3 Chapter: Modeling of Residual Stress Fields and Experimental Verification .... 58

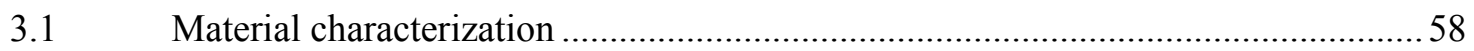

3.2 Test setup for the generation of a well-controlled residual stress field in a

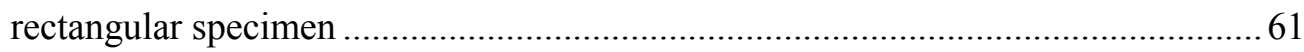

3.3 Elastic-plastic finite element model of the four point bending test .........................66

Results of strain/stress fields calculation ......................................................... 71 
3.5 Discussion of residual strain and stress fields due to four point bending test

simulation.

\section{Chapter: Numerical Model used to analyze the Fatigue Crack Growth in} Specimens with Residual Stresses ..................................................................... 85

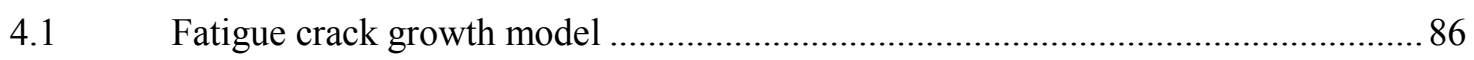

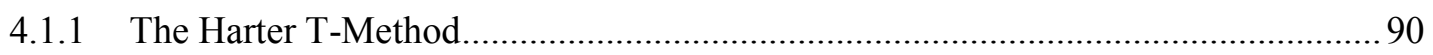

4.2 Finite element model for the fatigue crack growth test simulation ........................ 93

4.2.1 Focused mesh at the local domain of the crack and re-meshing strategy .................98

4.3 The effective stress intensity factor calculation by the displacement correlation

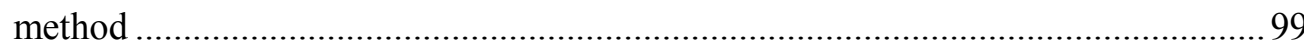

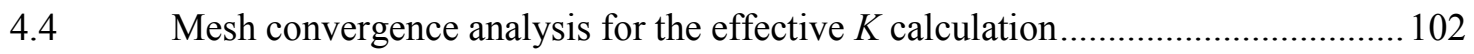

4.5 Finite element model for the effective $K$ calculation without the crack closure effect 105

4.5.1 Redistribution of residual stress fields during the fatigue crack growth without the crack closure effect 107

4.6 Finite element model for the effective $K$ calculation with the crack closure effect 113

4.6.1 Redistribution of residual stress fields during the fatigue crack growth with the crack closure effect......

4.7 Finite element model for the effective $K$ with the addition of crack closure and plastic wake effects.

4.7.1 Redistribution of residual stress fields during fatigue crack growth with the addition of the crack closure and plastic wake effects.

4.8 Test cases for the fatigue crack growth model 131

4.9 Experimental verification of the fatigue crack growth model 132

5 Chapter: Results and Discussion for the model of the Fatigue Crack Growth in Specimens with Residual Stress Fields. 
5.1 Finite element model for the effective $K$ calculation without the crack closure effect 135

5.1.1 Effective $K_{\max }, K_{\min }, \Delta K$ and $R$ for a large stress ratio (test case 1) ...................... 135

5.1.2 Effective $K_{\max }, K_{\min }, \Delta K$ and $R$ for a low stress ratio (test case 2) ........................ 138

5.1.3 Effective $K_{\max }, K_{\min }, \Delta K$ and $R$ for a negative stress ratio (test case 3) .................. 140

5.2 Finite element model for the effective $K$ calculation with the crack closure effect 143

5.2.1 Effective $K_{\max }, K_{\min }, \Delta K$ and $R$ for a large stress ratio (test case 1) ...................... 143

5.2.2 Effective $K_{\max }, K_{\min }, \Delta K$ and $R$ for a low stress ratio (test case 2) ........................ 144

5.2.3 Effective $K_{\max }, K_{\min }, \Delta K$ and $R$ for a negative stress ratio (test case 3) .................. 146

5.3 Finite element model for the effective $K$ calculation with the addition of the crack

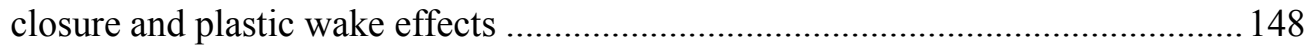

5.3.1 Effective $K_{\max }, K_{\min }, \Delta K$ and $R$ for a large stress ratio (test case 1) ....................... 148

5.3.2 Effective $K_{\max }, K_{\min }, \Delta K$ and $R$ for a low stress ratio (test case 2) ......................... 150

5.3.3 Effective $K_{\max }, K_{\min }, \Delta K$ and $R$ for a negative stress ratio (test case 3).................. 153

5.4 Fatigue crack growth rate and fatigue life calculations for test case 1 (nominal $R=$

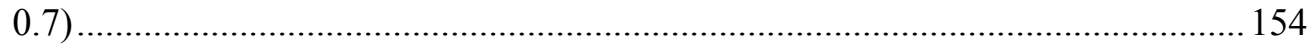

5.5 Fatigue crack growth rate and fatigue life calculations for test case 2 (nominal $R=$ $0.05)$ 165

5.6 Fatigue crack growth rate and fatigue life calculations for test case 3 (nominal $R=$ -

1) 174

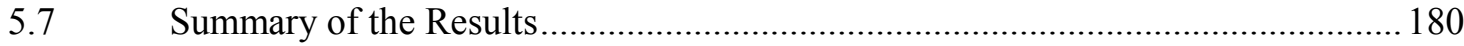

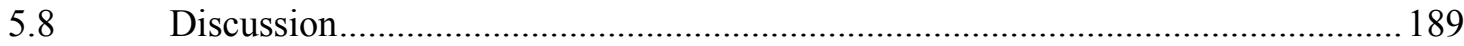

6 Chapter: Conclusions ................................................................................ 196

7 Chapter: Future work ............................................................................................. 200

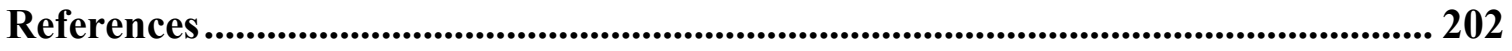




\section{List of Tables}

Table 3-1 Standard chemical composition of 7050-T7451 aluminum alloy expressed in

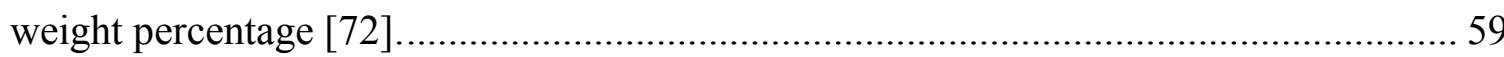

Table 3-2 Typical mechanical properties of 7050-T7451 aluminum alloy [72]............. 60

Table 3-3 Specimens, four-point bending test conditions and experimental methods. .... 65

Table 3-4 The material model parameters for elastic-plastic behavior of 7050-T7451

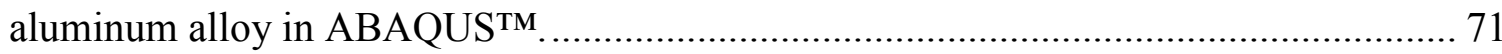

Table 3-5 Numerical and experimental results of the strain produced by the four-point

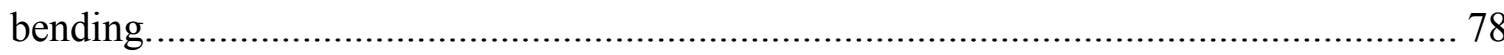

Table 3-6 Maximum and minimum values of the 1-D residual strain distribution along the mid-section of the test specimen (symmetry line on the unnotched-beam model).......... 79 Table 4-1 Test cases for numerical model of fatigue crack growth in residual stress fields

Table 5-1 Data results of the effective FCG parameters for the test case with a large stress ratio (nominal $R=0.7$ )

Table 5-2 Data results of the effective FCG parameters for the test case with a negative stress ratio $(R=-1)$

Table 5-3 Data results of the effective FCG parameters for the test case with a low stress ratio (nominal $R=0.05$ ) 152 Table 5-4 Summary of parameters required to model the FCG in components with a variable RSF. 195 


\section{List of Illustrations}

Figure 2-1 Microstructure for front- and back-faces of a test specimen made of austenitic stainless steel with a thickness of $3 \mathrm{~mm}$ and subjected to 12,000 loading cycles. (Reprinted from [16] under the CC BY-NC-ND license,

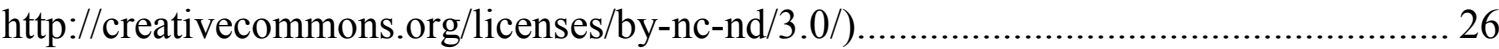

Figure 2-2 Theories to analyze the mechanical fatigue of engineering materials. 28

Figure 2-3 The type of residual stresses and the characteristic length scale over which they self-equilibrate. The type I $\left(\sigma_{\mathrm{rs}}{ }^{\mathrm{I}}\right)$ self-equilibrates over the dimensions of the component, the type II $\left(\sigma_{\mathrm{rS}}^{\mathrm{II}}\right)$ self-equilibrates over a few grains ( $\alpha$ and $\beta$ phases) and the type III $\left(\sigma_{\mathrm{rs}}^{\mathrm{III}}\right)$ self-equilibrates within the grain size. (Reprinted from [28]). 32 Figure 2-4 a) CT specimen extracted from the welded plate, and b) the fatigue crack growth rate for base and weld metals. (Reprinted from [35], with permission from Elsevier)

Figure 2-5 a) The back face strain versus the applied load for CT specimen (weld metal) and b) profiles of residual stresses, stresses due to minimum external load and their sum. (Reprinted from [35], with permission from Elsevier).

Figure 2-6 a) Residual strain measurements for a natural aged (NA) and an over aged (OA) Al matrix composite, and b) load vs back face strain during the four-point-bending of Al-matrix specimen with NA condition. (Reprinted from [6] with kind permission from Springer Science and Business Media. Copyright 1998 ASM International).......... 38 Figure 2-7 Crack closure level as a function of the crack length for: a) the natural aged (NA) condition and b) the over aged (OA) condition. [6]. (Reprinted from [6], with kind permission from Springer Science and Business Media). 
Figure 2-8 a) Details of initial cracks machined in cold-hole expanded specimens; b) the fatigue crack growth behavior for a non-cold expanded hole with the Paris-Erdogan relationship fit for short and long cracks, and the fatigue crack growth rate as a function of the crack length for: c) $R=0.7$ and $\sigma_{\max }=162 \mathrm{MPa}$ and d) $R=0.1$ and $\sigma_{\max }=162$ MPa. (Reprinted from [46], with permission from Elsevier).

Figure 2-9 Fatigue crack growth results for a single tensile overload: a) The crack length $a$ vs the number of cycles $N$, and b) the fatigue crack growth rate $d a / d N$ vs the stressintensity factor range $\Delta K$. Six specimens were prepared at different crack-growth stages to characterize the residual stresses by the neutron diffraction technique. (Reprinted with permission from [47]. Copyright 2010, AIP Publishing LLC.) 42 Figure 2-10 Residual stresses as a function of the distance from the overload point for specimens: a) SP1 \& SP2, b) SP2 \& SP4, and c) SP4 \& SP6. (Reprinted with permission from [47]. Copyright 2010, AIP Publishing LLC.) 44 Figure 2-11 Pre-existing residual stresses in an uncracked specimen: a) Single-Edge Bend $\mathrm{SE}(\mathrm{B})$, and b) cold-hole expanded. (a) Reprinted from [59], with permission from John Wiley and Sons; Copyright 2008 Blackwell Publishing Ltd. (b) Reprinted from [56] with permission from Elsevier; Copyright 2009 Elsevier. 48 Figure 2-12 Predictions and measurements of: a) the fatigue crack growth rate, and b) the fatigue life. (Reprinted from [59], with permission from John Wiley and Sons; Copyright 2008 Blackwell Publishing Ltd.) 49

Figure 2-13 Predictions and measurements of fatigue life for cold-hole expanded specimen. (Reprinted from [56] with permission from Elsevier; Copyright 2009 Elsevier.) 
Figure 2-14 a) The stress intensity factor as a function of the applied stress for plain and cold expanded holes with a $2 \mathrm{~mm}$ diameter. The fatigue crack growth rate as a function of the crack length for: c) $R=0.7$ and $\sigma_{\max }=162 \mathrm{MPa}$ and d) $R=0.1$ and $\sigma_{\max }=162 \mathrm{MPa}$. (Reprinted from [46] with permission from Elsevier. Copyright 2003)...... 52 Figure 2-15 a) FEA model mesh, b) FEA results of the crack opening stress as a function of the crack length, c) the experimental and NASGRO fatigue crack growth curves, and d) the predictions of fatigue life. (Reprinted from [8] with permission from Elsevier. Copyright 2006 Elsevier Ltd.) 55

Figure 3-1 Engineering stress-strain curve for 7050-T7451 aluminum alloy. 61 Figure 3-2 Pre-existing residual stress fields in a 7050-T7451 aluminum plate. (Reprinted from [75] ) 62 Figure 3-3 Four-point bending test setup and unnotched-beam specimen dimensions. ... 63 Figure 3-4 Finite element model to compute the residual stress fields...... 67 Figure 3-5 a) Isotropic hardening rule; b) kinematic hardening rule; c) combined hardening rule; and d) yield points under stress reversal for each hardening rule.

Figure 3-6 True stress-strain curve and material data points for the combined hardening model used in the FEA models.

Figure 3-7 Strain field produced by the maximum applied load during the four-point bending test according to the FEA simulation and the experimental measurement: a) unnotched-beam model and b) DIC technique on test specimen B3. 72 Figure 3-8 Residual strain field after the four-point bending test: a) unnotched-beam model and b) DIC technique on test specimen B3 
Figure 3-9 1-D distribution of residual strain in the unnotched-beam model (FEA analysis) and test specimens B2, B3 and B4 (DIC technique). Strain point measurements with Strain Gauges (SG) on test specimens B2,B3 and B4. 74 Figure 3-10 Residual elastic strain component according to the finite element results.... 75 Figure 3-11 Residual plastic strain component according to the finite element results. .. 75 Figure 3-12 a) Contour plot of residual stresses (MPa) produced by the four-pointbending test simulation, and $b$ ) the residual stress field distribution (1-D) as a function of the $y$-coordinate along the plane of symmetry on the unnotched-beam model. 76 Figure 3-13 Comparison of RSF determined from the FEA model with an initial yield point at $0.025 \%$ and $0.2 \%$ proof stress.

Figure 3-14 The yield stress defined at: a) $0.2 \%$ and b) $0.025 \%$ proof stress. 84 Figure 4-1 Baseline fatigue crack growth curves for 7050-T7451 aluminum alloy at $R=$ $0.7,0.05$ and -1 . 89 Figure 4-2 Harter T-method for interpolation of baseline $d a / d N$ vs. $\Delta K$ curves [51]....... 91 Figure 4-3 Experimental fatigue crack growth data points used to compute the exponent $m_{i}$ in the Harter T-method, and new interpolated baseline curves for $R=0.6,0.4,0.2,-0.5$ and -0.7 93 Figure 4-4 SENT specimen for the fatigue crack growth model with a schematic plot of pre-existing residual stresses produced by four-point bending. 94 Figure 4-5 a) Half symmetry FEA model of SENT specimen and grip part for the fatigue crack growth test simulation, b) mesh detail of the specimen with a crack $a_{0}=5.08 \mathrm{~mm}$, and c) clamping of the specimen by the grip part. 96 
Figure 4-6 a) Focused mesh around the crack tip region, and b) re-meshed for the crack lengths $a_{5}=7.82, a_{15}=12.7, a_{25}=17.78$ and $a_{35}=22.86 \mathrm{~mm}$. 98

Figure 4-7 The displacement correlation technique to calculate the effective stress intensity factor. 100

Figure 4-8 Element sizes for the mesh convergence analysis of the effective $K$ calculation: a) $0.127 \mathrm{~mm}$ and b) $0.056 \mathrm{~mm}$. 102 Figure 4-9 Displacement fields (mm) around the crack tip in the SENT specimen under mode I opening load: a) element size of $0.127 \mathrm{~mm}$ and b) element size of $0.056 \mathrm{~mm}$. . 103 Figure 4-10 Nodal displacements behind the crack tip for element sizes of $0.127 \mathrm{~mm}$ and $0.056 \mathrm{~mm}$. 104

Figure 4-11 Effective stress-intensity factor $K$ according to element sizes of $0.127 \mathrm{~mm}$ and $0.056 \mathrm{~mm}$. 105

Figure 4-12 Flowchart of the FCG model to calculate the fatigue crack growth rate and fatigue life. 106

Figure 4-13 Residual stress fields $\left(\mathrm{S}_{11}\right.$ in $\left.\mathrm{MPa}\right)$ in SENT model without the crack closure effect for crack lengths: a) $\left.\left.a_{0}=5.08 \mathrm{~mm}, \mathrm{~b}\right) a_{5}=7.62 \mathrm{~mm}, \mathrm{c}\right) a_{15}=12.7 \mathrm{~mm}$. d) Preexisting residual stresses in the unnotched-beam model, which was the initial condition for the fatigue crack growth test simulation. 109 Figure 4-14 Distribution of residual stresses (1-D) along the fatigue crack growth plane in the SENT model without the crack closure effect: a) fatigue crack lengths $a_{0}=5.08 \mathrm{~mm}$, $a_{5}=7.62 \mathrm{~mm}$ and $a_{10}=10.16 \mathrm{~mm}$ with a closer view in $\mathrm{b}$ ), and c) fatigue crack lengths $a_{15}=12.7 \mathrm{~mm}, a_{25}=17.78$ and $a_{35}=22.86 \mathrm{~mm}$ with a closer view in d). Pre-existing residual stresses are also plotted for reference. 
Figure 4-15 The finite element model for the effective $K$ calculation with addition of the crack closure effect. 114

Figure 4-16 Residual stress fields $\left(\mathrm{S}_{11}\right.$ in $\left.\mathrm{MPa}\right)$ from the SENT model with the crack closure effect for fatigue crack lengths: a) $a_{4}=7.112 \mathrm{~mm}$, b) $a_{9}=9.652 \mathrm{~mm}$ and c) $a_{19}=14.732 \mathrm{~mm}$. d) Pre-existing residual stresses in the unnotched-beam model, which was the initial condition for the fatigue crack growth test simulation. 116 Figure 4-17 Comparison of the redistribution of residual stresses for the SENT model with fatigue crack length $a_{25}=17.78 \mathrm{~mm}$ according to: a) SENT model without the crack closure effect, and b) SENT model with the crack closure effect. 117 Figure 4-18 Distribution of residual stresses (1-D) along the fatigue crack growth plane in the SENT model with the crack closure effect: a) Fatigue crack lengths $a_{0}=5.08 \mathrm{~mm}, a_{5}$ $=7.62 \mathrm{~mm}$ and $a_{10}=10.16 \mathrm{~mm}$ with a closer view in $\mathrm{b}$ ), and c) fatigue crack lengths $a_{15}$ $=12.7 \mathrm{~mm}, a_{25}=17.78$ and $a_{35}=22.86 \mathrm{~mm}$ with a closer view in d). Pre-existing residual stresses are also plotted for reference.

Figure 4-19 Comparison of residual stresses along the fatigue crack growth between the SENT models without the crack closure effect (Effective $K$ ) and with the crack closure effect (Effective K + Crack Closure). Crack lengths a) $\left.a_{15}=12.7 \mathrm{~mm}, \mathrm{~b}\right) a_{25}=17.78 \mathrm{~mm}$ and c) $a_{35}=22.86 \mathrm{~mm}$.

Figure 4-20 Focused mesh in the SENT model with the addition of the crack closure and plastic wake effects. 122

Figure 4-21 Residual stress fields from the SENT model with the addition of the crack closure and plastic wake effects for fatigue crack lengths: a) $a_{6}=8.128 \mathrm{~mm}$, b) $a_{16}=$ 
$13.208 \mathrm{~mm}$ and c) $a_{26}=18.288 \mathrm{~mm}$. d) Pre-existing residual stresses after the four-point bending test simulation (unnotched-beam model) 125

Figure 4-22 Distribution of residual stresses (1-D) along the fatigue crack growth plane in the SENT model with the incorporation of the crack closure and plastic wake effect: a) Crack length configurations $a_{0}=5.08 \mathrm{~mm}, a_{5}=7.62 \mathrm{~mm}$ and $a_{10}=10.16 \mathrm{~mm}$ with a closer view in b), and c) crack length configurations $a_{15}=12.7 \mathrm{~mm}, a_{25}=17.78$ and $a_{35}=$ $22.86 \mathrm{~mm}$ with a closer view in d). Pre-existing residual stresses after the four-point bending test simulation.

Figure 4-23 Comparison of residual stresses along the fatigue crack growth plane in the SENT model (zero external loads) between the SENT model with the addition of the crack closure and plastic wake effects, and the SENT model with only crack closure effect. Fatigue crack lengths a) $\left.a_{0}=5.08 \mathrm{~mm}, \mathrm{~b}\right) a_{5}=7.62 \mathrm{~mm}$, c) $a_{10}=10.16 \mathrm{~mm}$ and d) $a_{15}=12.7 \mathrm{~mm}$.

Figure 4-24 Redistribution of residual stress fields (zero external loads) for SENT model with crack length $a_{0}=5.08 \mathrm{~mm}$ as a function of the element size around the crack tip: a) $56 \mu \mathrm{m}$ and b) $127 \mu \mathrm{m}$ 130

Figure 5-1 Effective stress intensity and stress ratio parameters for a large stress ratio without the crack closure effect: a) $K_{\max }, K_{\min }$ and $\Delta K$, and b) $R$. 136 Figure 5-2 Effective stress intensity and stress ratio parameters for a low stress ratio without the crack closure effect: a) $K_{\max }, K_{\min }$ and $\Delta K$, and b) $R$ 138 Figure 5-3 Effective stress intensity and stress ratio parameters for a negative stress ratio without the crack closure effect: a) $K_{\max }, K_{\min }$ and $\Delta K$, and b) $R$ 140 
Figure 5-4 Effective stress intensity and stress ratio parameters for a low stress ratio with the crack closure effect: a) $K_{\max }, K_{\min }$ and $\Delta K$, and b) $R$

Figure 5-5 Effective stress intensity and stress ratio parameters for a negative stress ratio with the crack closure effect: a) $K_{\max }, K_{\min }$ and $\Delta K$, and b) $R$

Figure 5-6 Effective stress intensity and stress ratio parameters for a large stress ratio with the addition of the crack closure and plastic wake effects: a) $K_{\max }, K_{\min }$ and $\Delta K$, and b) $R$. 149

Figure 5-7 Effective stress intensity and stress ratio parameters for a low stress ratio with the addition of the crack closure and plastic wake effects: a) $K_{\max }, K_{\min }$ and $\Delta K$, and b) $R$.

Figure 5-8 Effective stress intensity and ratio parameters for a negative stress ratio with the addition of the crack closure and plastic wake effects: a) $K_{\max }, K_{\min }$ and $\Delta K$, and b) $R$.

Figure 5-9 Fatigue crack growth rate as a function of the crack length for test case 1 with a large stress ratio $\left(P_{\max }=55 \mathrm{kN} ; R=0.7 ; \mathrm{C} 11 \mathrm{~B} 10\right.$ specimen $)$ : a) Full range of crack lengths, and b) range of crack lengths from $a_{6}=6.096 \mathrm{~mm}$ to $a_{23}=16.764 \mathrm{~mm}$. 156 Figure 5-10 Crack profile during the fatigue crack growth test simulation for test case 1 at $R=0.7: \mathrm{a})$ maximum applied load $\left(P_{\max }=55 \mathrm{kN}\right)$ and $\left.\mathrm{b}\right)$ minimum applied load $\left(P_{\min }=\right.$ $38.5 \mathrm{kN})$. 158

Figure 5-11 Fatigue crack growth rate and residual stresses at different crack lengths for the SENT model with a large stress ratio. 160 
Figure 5-12 The stress distribution (1-D) for fatigue crack lengths $a_{5}=7.62 \mathrm{~mm}, a_{10}=$ $10.16 \mathrm{~mm}$ and $a_{15}=12.7 \mathrm{~mm}$ in the SENT model with the minimum applied cyclic load $\left(P_{\min }=38.5 \mathrm{kN}\right)$ and a large stress ratio $(R=0.7)$. 162

Figure 5-13 Crack length versus number of cycles for test case 1 (C11B10 specimen, $P_{\max }$ $=55 \mathrm{kN}$ and $R=0.7)$

Figure 5-14 Fatigue crack growth rate as a function of the crack length for test case 2 (C5B3 specimen, $P_{\max }=55 \mathrm{kN}$ and $R=0.7$ ): a) Full range of crack lengths, and b) crack lengths $a_{6}=6.096 \mathrm{~mm}$ to $a_{23}=16.764 \mathrm{~mm}$. 167

Figure 5-15 Fatigue crack growth rate and residual stresses at different crack lengths for the SENT model with a low stress ratio. 170

Figure 5-16 The stress distribution (1-D) for fatigue crack lengths $a_{5}=7.62 \mathrm{~mm}, a_{10}=$ $10.16 \mathrm{~mm}$ and $a_{15}=12.7 \mathrm{~mm}$ in the SENT model with the minimum applied cyclic load $\left(P_{\min }=1.75 \mathrm{kN}\right)$ and a low stress ratio $(R=0.05)$.

Figure 5-17 Crack length versus number of cycles for test case 2 (C5B3 specimen, $P_{\max }=$ $35 \mathrm{kN}$ and $R=0.05)$. 172

Figure 5-18 Fatigue crack growth rate as a function of the crack length for test case 3 (C10B8 specimen, $P_{\max }=35 \mathrm{kN}$ and $R=-1$ ): a) Full range of crack lengths, and b) crack lengths $a_{6}=6.096 \mathrm{~mm}$ to $a_{23}=16.764 \mathrm{~mm}$. 175

Figure 5-19 Fatigue crack growth rate and residual stresses at different crack lengths for the SENT model with a negative stress ratio. 177 Figure 5-20 The stress distribution (1-D) for fatigue crack lengths $a_{5}=7.62 \mathrm{~mm}, a_{10}=$ $10.16 \mathrm{~mm}$ and $a_{14}=12.192 \mathrm{~mm}$ in the SENT model with the minimum applied cyclic load $\left(P_{\min }=-35 \mathrm{kN}\right)$ and a low stress ratio $(R=0.05)$ 178 
Figure 5-21 Crack length versus number of cycles for test case 3 (C10B8 specimen, $P_{\max }$ $=35 \mathrm{kN}$ and $R=-1)$.

Figure 5-22 Prediction ratio of the fatigue crack growth rate according to the three SENT models. 181

Figure 5-23 Crack length profile during fatigue crack growth test simulation of test case 2 with a low stress ratio: a) Maximum applied cyclic load $\left(P_{\max }=35 \mathrm{kN}\right)$ and b) minimum applied cyclic load $\left(P_{\min }=1.75 \mathrm{kN}\right)$. 183

Figure 5-24 Crack front profile during the fatigue crack growth test simulation of test case 2 with a low stress ratio. 184 Figure 5-25 Distribution of the plastic wake for specimen C5B3 $\left(P_{\max }=35 \mathrm{kN}\right.$ and $R=$ 0.05) during the FCG simulation. Crack length configurations: a) $a_{2}=6.096 \mathrm{~mm}, a_{5}=$ $7.62 \mathrm{~mm}, a_{7}=8.636 \mathrm{~mm}, a_{10}=10.16 \mathrm{~mm}$; b) $a_{10}=10.16 \mathrm{~mm}, a_{13}=11.684 \mathrm{~mm}, a_{15}=$ $12.7 \mathrm{~mm}, a_{18}=14.224 \mathrm{~mm}$; and c) $a_{18}=14.224 \mathrm{~mm}, a_{20}=15.24 \mathrm{~mm}, a_{25}=17.78 \mathrm{~mm}$ and $a_{35}=22.86 \mathrm{~mm}$. The residual plastic strain field produced by the four-point bending simulation is shown for reference (Pre-existing; dotted red line). 186 Figure 5-26 Yield state of the finite elements for several crack lengths subjected to $P_{\max }=$ $35 \mathrm{kN}$ (test case 2), according to SENT model with the addition of the crack closure and plastic wake effects. 188 


\section{Chapter: Introduction}

This manuscript focuses on the mutual effects of the Fatigue Crack Growth (FCG) phenomenon and Residual Stress Fields (RSF). FCG is an irreversible physical process, which can be a recurrent condition for machines, vehicles and structures in service. In general, manufactured components are inhomogeneous at the microstructural scale due to the presence of pores, cracks and inclusions. Upon the action of cyclic loads, pores increase their size and coalesce to form micro-cracks. The micro-cracks can grow and connect with each other until a large crack is created, which, depending on the loading conditions, can grow until it exceeds a critical size, thus decreasing the structural integrity of the component. Perhaps, the most common cause of fracture in engineering components is the FCG phenomenon [1]. There are no accurate estimates of the number of mechanical failures associated with FCG phenomena, however, an old report indicated that 141 out of 230 mechanical failures presented in the United States in 1982 were due to FCG phenomena. In addition, it was indicated in the same report that the aircraft and motor vehicles sectors were the most affected by the FCG phenomenon [1].

In the 1950s, the fail-safe design philosophy emerged as a result of the catastrophic failures of the British DeHavilland Comet, which was the first commercial jetliner. The design of square shape windows in The DeHavilland Comet created a stress concentration. This square window in The DeHavilland Comet in combination with imperfections introduced by the punch rivet technique employed to install the windows in the cabin, led to a FCG failure resulting in the Comet fatalities [2,3]. Almost 20 years after the introduction of The DeHavilland Comet, the United States Air Force (USAF) 
created a damage tolerance design philosophy that assumed the presence of initial damage in the materials and thus ensured aircraft structural integrity. It was later recognized that multiple load paths and residual strength concepts employed by the failsafe design philosophy were not enough to ensure aircraft structural integrity. This understanding was developed as a consequence of a fatal incident on the F-111 aircraft No. 94, which developed a fatigue crack of large dimensions $(23.4 \mathrm{~mm} \times 5.9 \mathrm{~mm})$ from an undetected material flaw. The airframe test programmes that were conducted to define a specific solution for the safe operation of the F-111 fleet, and the recurrent FCG issues presented in the Lockheed C5-a wing boxes also contributed to the development of the damage tolerance design philosophy [3].

Residual stresses introduced during the manufacturing of mechanical components can superimpose over stresses produced by external loads and thus decrease or increase their fatigue life. In general, tensile residual stresses in mechanical components decrease the fatigue life and may promote the occurrence of unforeseen catastrophic failures. On the contrary, compressive residual stresses in mechanical components tend to increase their fatigue life and as such, may be deliberately introduced into materials. A routine practice employed by several industries is to introduce compressive residual stresses to critical components or regions of the component. However, these beneficial effects are not taken into account during the design process since there are several other challenges to overcome. From a damage tolerant perspective, it is necessary to develop reliable models to accurately predict the fatigue life of mechanical components with residual stresses. Furthermore, it is not an easy task to introduce the same level of residual stresses into the machined components on a production line. It is even more challenging to ensure through 
measurements that the specified residual stress field has been actually introduced into the machined component and to monitor its behavior under operational conditions. [4] The present doctoral research addresses the following issues: i) modeling RSF with the FEA method generated by inhomogeneous plastic deformation in the 7050-T7451 aluminum alloy, ii) analysis of the mutual effects between the FCG phenomenon and RSF, iii) development of an FCG model to simulate the FCG process and evaluate fatigue life of the specimens with residual stresses and iv) experimental verification of computer simulation results.

Several mechanical processes may be employed to generate a RSF such as, for example, welding, casting, heat treatment, shot peening, but as previously mentioned, it is difficult to control and ensure the development of a well-defined, consistent and repeatable RSF in the component by these methods. A four-point bending test operation was used in this research for a consistent analysis of the variable RSF, which consisted of well-defined tension and compression regions. This operation generated an inhomogeneous plastic deformation in the specimens, thus ensuring good control of the RSF used in the FCG analysis. Non-uniform plastic deformation induced by other manufacturing techniques is more complex to control in part due to a large number of processing variables.

The redistribution of RSF resulting from the FCG process and the plastic deformation at the crack tip is usually not considered by current methodologies used to predict the FCG in specimens with residual stresses $[5,6,7]$. Recently, the FEA method has been used to model plasticity-induced fatigue crack closure in order to determine the crack opening stress. The crack opening stress corresponds to the applied load to the component that first opens the crack faces. The crack opening stress determined by the FEA method may 
be used to predict the FCG through RSF in conjunction with empirical relationships of the FCG rate versus the effective stress-intensity factor ( $d a / d N$ vs $\Delta K_{\text {eff }}$ [8]. However, the empirical relationship $d a / d N$ vs $\Delta K_{\text {eff }}$ is based on experimental measurements of the crack closure effect that are difficult to accomplish and highly dependent on the measurement system [9]. Indeed, there is not a measurement standard to determine the crack closure during the FCG phenomena.

In this work, a commercially available FEA program (ABAQUS) was used to simulate the four-point bending treatment and the FCG test. The simulation included the creation of a variable RSF with well-defined tension and compression regions. This variable RSF was used as an initial condition for the FCG test simulation. An unnotched beam specimen with a rectangular geometry was used as the base model to be analyzed during the four-point bending test simulation; it was then modified into a Single-Edge Notched Tension (SENT) specimen for the FCG test simulation. A FCG model was developed to determine the fatigue life of the SENT specimens with a prescribed variable RSF. The FCG model was based on the FEA simulations and experimental baseline FCG curves. The main questions to be addressed by the present research are:

1. How are the residual stresses in the SENT specimen redistributed due to the FCG, and how does the actual RSF affect the FCG under different stress ratios $(R=0.7$, 0.05 and -1 ) from qualitative and quantitative perspectives?

2. What is the contribution of the crack closure and plastic wake effects to the FCG through RSF, and how does the RSF affect the plastic zone developed during the FCG? 
Finally, the FCG phenomena through RSF analyzed in this manuscript is limited to fatigue tests under constant-amplitude loading and to macro-crack growth. However, it is also important to analyze the effect of RSF on the fatigue crack initiation phenomenon, but this was out of the scope of this manuscript.

\subsection{Structure of the thesis}

The following paragraphs contain a general description of the material presented in this manuscript. In general, the chapters may be read as standalone sections with the exception of chapter 5 that is a continuation to chapter 4 .

Chapter 1 provides an overview of the FCG phenomena and RSF, and their implications for mechanical components in service. In addition, the focus of the research is presented along with a general description of the numerical model developed in this research to analyze the FCG in the specimens with residual stresses.

Chapter 2 presents the background for the FCG, the RSF and their interaction, along with an outline and description of the design philosophies against fatigue failures. In addition, experimental and numerical methods employed in the literature for the analysis of FCG through RSF are reviewed. At the end of this chapter, the research objectives of this manuscript are presented based on final remarks from the literature review.

Chapter 3 describes a simulation of a four-point bending test operation over a rectangular specimen to generate a RSF. The base material (7050-T7451 aluminum alloy) employed in this research is presented along with the dimensions and details of the rectangular specimen, in addition to the four-point bending test set-up used for the introduction of a 
controlled RSF. Also, the constitutive model employed in this research for the elasticplastic material behavior is presented, in conjunction with the residual stress and strain fields determined by the commercially available finite element software ABAQUS ${ }^{\mathrm{TM}}$. Finally, numerical results are verified against the experimental measurements of residual stress and strain fields determined by the slitting method and the digital image correlation technique.

Chapter 4 presents a numerical model for the analysis of the FCG in specimens containing residual stresses. The experimental curves that were determined from the standard FCG tests on the aluminum alloy 7050-T7451 are also presented, along with the Harter T-method and the FEA model used for the FCG test simulations. A mesh convergence analysis is presented for the calculation of the effective $K$. In addition, the re-meshing strategy and incorporation of the crack closure and plastic wake effects into the FE model are discussed, and different FCG test case scenarios analyzed in this manuscript are presented. Finally, the analysis of the redistribution of RSF due to FCG is also presented.

Chapter 5 presents and discusses the results of the numerical model presented in the previous chapter. The results of the calculation of the FCG model parameters (effective $K$ and $R$ ) are presented for each test case analyzed in conjunction with the FCG rate and fatigue life predictions. The crack closure and plastic wake effects in the FCG model are also analyzed and discussed. Finally, the numerical results are verified against the experimental FCG tests conducted in concordance with the dimensions, material and general conditions employed in the simulations presented in this manuscript. 
Chapters 6 and 7 present the conclusions of the study and the recommendations for future research work. 


\section{Chapter: Background and literature review}

The Fatigue Crack Growth (FCG) and the Residual Stress Fields (RSF) are common topics of research in the literature, due to their importance for a large number of engineering applications. A search of scientific databases for each of these topics results in thousands of publications per year. However, if both topics are combined in the search, in order to understand their mutual effects, then just a few publications are found per year.

This literature review chapter is structured to present a physical description of the FCG phenomena. Design philosophies for the prevention of fatigue failures are presented next, along with the implications of the residual stress fields for design of mechanical components. In addition, a review of experimental methods and numerical models for the FCG in specimens containing residual stresses is presented with the final remarks leading to the scientific objectives of the study presented in this manuscript.

\subsection{The physics of the fatigue crack growth phenomena}

The metal fatigue process is a cumulative physical damage caused by cyclic stresses or deformations, which is irreversible and may lead to a macro structural failure. From a microscopic perspective, most of the metallic materials are aggregates of crystals and defects so that the applied cyclic stresses are not uniformly distributed at the microstructural scale. The physical damage is more severe around microstructural inhomogeneities, where cracks nucleate from the pre-existing voids, inclusions, slip 
bands, grain boundaries, scratches or sharp flaws; afterwards, the cracks increase in size and link up with other cracks until a critical size is reached. Subsequently, the crack rapidly propagates in an unstable way towards a sudden and catastrophic final failure. $[10,11,12,13,14,15]$. For instance, Figure $2-1$ presents the microstructure for the frontand back-faces of a test specimen (thickness of $3 \mathrm{~mm}$ ) made of an austenitic stainless steel, where a crack is observed as a consequence of the application of 12,000 loading cycles [16]. The crack nucleates and grows from a stress concentration zone. Different crack lengths and propagations paths were observed between the front- and back-sides of the same specimen, as a consequence of microstructural inhomogeneities such as pores, voids and grain boundaries.

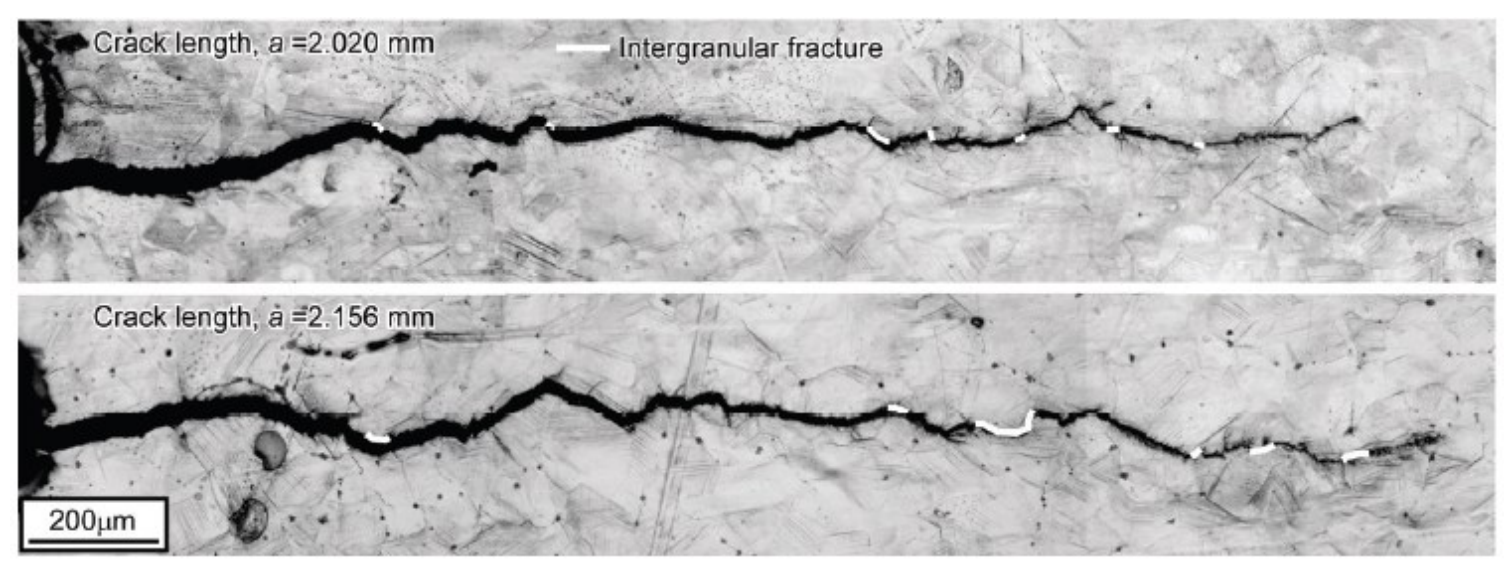

Figure 2-1 Microstructure for front- and back-faces of a test specimen made of austenitic stainless steel with a thickness of $3 \mathrm{~mm}$ and subjected to 12,000 loading cycles. (Reprinted from [16] under the CC BY-NC-ND license, http://creativecommons.org/licenses/by-nc-nd/3.0/).

The fatigue research has been strongly motivated by the occurrences of mechanical failures. A historical analysis shows that major catastrophic failures in past years, such as 
The World War II Liberty ships [17], The DeHavilland Comet [2], The Aloha Airline Boeing 737 [18] and The Missouri Air National Guard F-15C [19] resulted in large research funding provided by governments and industries in order to achieve a greater understanding of the fatigue phenomena and introduce safer design methodologies. Indeed, most of these accidents have led to modifications and/or introduction of new structural fatigue design philosophies.

\subsection{Design philosophies for the avoidance of fatigue failures}

Figure 2.2 presents an outline for the analysis of fatigue processes according to the stressbased, strain-based and fracture mechanics theories. The $S$ - $N$ curves are employed in the infinite and safe life design philosophies, while $\varepsilon-N$ curves are only employed in the safe life design philosophy. The damage tolerant design philosophy is based on the fracture mechanics theory and employs the $d a / d N-\Delta K$ curves [20]. The next paragraphs briefly describe these design philosophies in more detail. The final paragraph briefly discusses the implications of the RSF for the fatigue processes, prior to providing the background information on the RSF in the next section.

The oldest design philosophy is the infinite life one, wherein the engineering component is designed to support service stresses below the fatigue limit or the fatigue strength of the material, that is, for an infinite life or an arbitrary number of cycles [10]. The fatigue limit or strength is characterized via the $S$ - $N$ curves. Experimental fatigue tests with constant amplitude stresses are conducted to determine the number of cycles required for complete failure of the test specimen. Several specimens are tested with the same 
procedure, but at different amplitude stress levels in order to plot an entire $S-N$ curve. The ASTM standard E466 provides detailed information on how to conduct the experimental fatigue test in order to determine the $S-N$ curves for metallic materials [21]. The fatigue limit for steels and titanium alloys [22] is defined as the stress amplitude at which the $S-N$ curve presents a plateau (Figure 2-2); below this stress limit the material does not present fatigue failures $[10,2]$. Other materials such as aluminum, magnesium and copper alloys do not present a plateau [22] and a magnitude of the fatigue strength is defined instead of the fatigue limit. The fatigue strength is simply defined as the stress amplitude at a specified number of the cycles to failure [10].

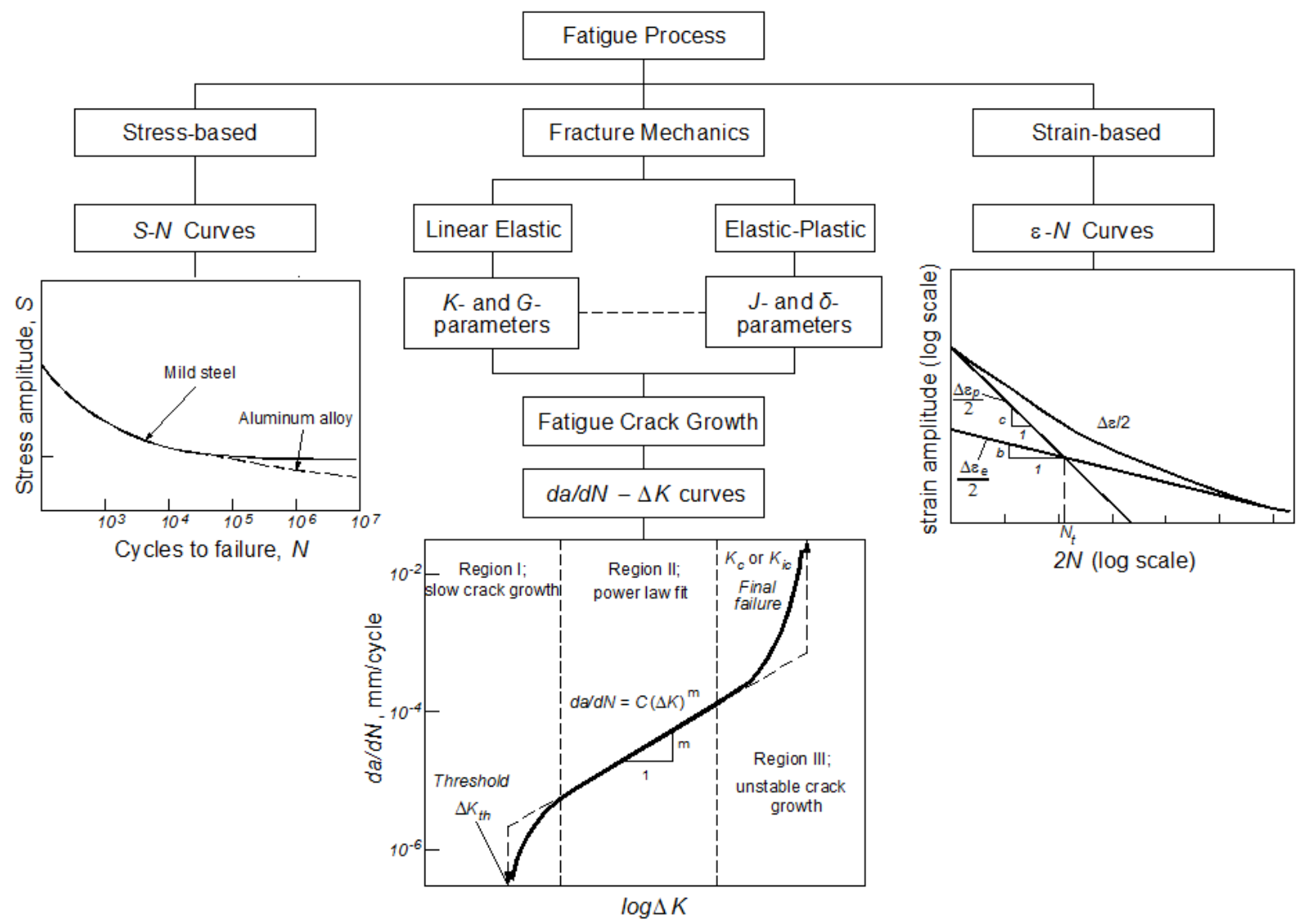

Figure 2-2 Theories to analyze the mechanical fatigue of engineering materials. 
The safe life design philosophy is used to determine the number of cycles required to develop a critical crack [20]. It employs strain and stress life curves ( $\varepsilon$ vs $N$ and $S$ vs $N$ ). The $\varepsilon$ vs $N$ curves are determined by experimental fatigue tests under the conditions of controlled strain and under a cyclic load that is completely reversed, i.e. at fixed amplitude strain and the mean stress equal to zero [10]. The mean stress is defined as the average of the maximum and minimum cyclic stress. Several test specimens are required to determine an entire $\varepsilon$ vs $N$ curve, which plots the total, elastic and plastic strain amplitudes against the number of complete reversals (cycles) as presented in Figure 2-2. The Coffin-Manson empirical relationship [23] is widely employed in the safe life design philosophy to characterize the strain life curves for metallic materials. The combination of the Neuber's rule [24], which relates the stress and strain concentration factors for local yielding at notches, and the Coffin-Manson relationship are employed to predict the number of cycles to crack initiation [20].

The damage tolerant design philosophy assumes the presence of fatigue cracks on the engineering component and employs empirical relationships of the FCG rate versus the stress intensity factor range ( $d a / d N$ vs $\Delta K$ curves) to predict a residual life [20]. An experimental FCG test of a standard specimen with a sharp crack is conducted normally under constant amplitude load and specific stress ratio [25]. The stress ratio is defined as the minimum over the maximum cyclic loading stresses. During the FCG test, the crack length is recorded against the number of cycles ( $a$ vs $N$ curve) and the FCG rate is defined as the ratio of change in crack length with respect to the number of cycles $(d a / d N)$. Closed form solutions for standard specimens are employed to compute the stress intensity factor $(K)$ as a function of the crack length, geometry and applied load. 
The difference between the maximum and minimum stress intensity factors applied during the FCG test correspond to $\Delta K$. In the fracture mechanics theory for a given geometry and external loads, the stress fields at the crack tip are dependent on a single parameter, e.g. the stress intensity factor $K$ or the energy release rate $G$ parameters for linear elastic materials, or the $J$ contour integral or the crack tip opening displacement $\delta$ for elastic-plastic materials. The $d a / d N$ vs. $\Delta K$ curves on logarithmic scales show a sigmoidal function with three regimes (Figure 2-2); the FCG threshold $\Delta K_{t h}$ where the fatigue crack has no growth, the final failure region at high FCG rates where the crack growth becomes unstable and a final fatigue failure arises, and the intermediate linear region which is represented by the well-known Paris-Erdogan relationship.

A common classification for metal fatigue processes is High Cycle Fatigue (HCF) and Low Cycle Fatigue (LCF). The LCF cases apply to engineering components subjected to high stress amplitudes that are above the yield strength of the material. The components experience considerable plastic deformations during the cyclic loading. The RSF have a low impact in LCF cases, because large plastic deformations relax any pre-existing residual stresses [26]. There is not a specific number of cycles to separate the LCF from HCF cases. However, the HCF cases involve service loads that are limited to the elastic range of the material. The service stresses by themselves would not cause plastic deformation. The pre-existing residual stresses in engineering components subjected to the HCF cases have a significant impact on the component's fatigue life, because the residual stresses influence the mean stress [26]. 


\subsection{Residual stress fields}

A typical feature of most manufactured or fabricated components is the presence of residual stresses that balance to zero over a characteristic length scale [27]. Three types of residual stresses exist: a) the residual stresses of type I, which balance to zero over large distances and are constant at the microstructural level with the characteristic length scale of the same order of magnitude as the component's dimensions (e.g., the stress field produced by the plastic bending of a bar); b) the residual stresses of type II, which balance to zero over short distances (a few grains) and are not constant at the microstructural level with the characteristic length of the same order of magnitude as the dimension of the grains (e.g., stresses in composite materials due to different coefficients of thermal expansion between the matrix and the fibre reinforcement); c) the residual stresses of type III, which balance to zero within a single grain and are meaningless at the microstructural level because they are relevant for the crystalline structure (e.g., stresses due to point defects, dislocations and coherency at interfaces). Figure 2-3 illustrates the three types of residual stresses and their associated characteristic length scales for a multiphase material ( $\alpha$ - and $\beta$-phase) [28]. Residual stresses of type I are indicated in the figure as $\sigma^{\mathrm{I}}$, those of type II are indicated as $\sigma^{\mathrm{II}}$ and those of type III are indicated as $\sigma^{\mathrm{III}}$. 


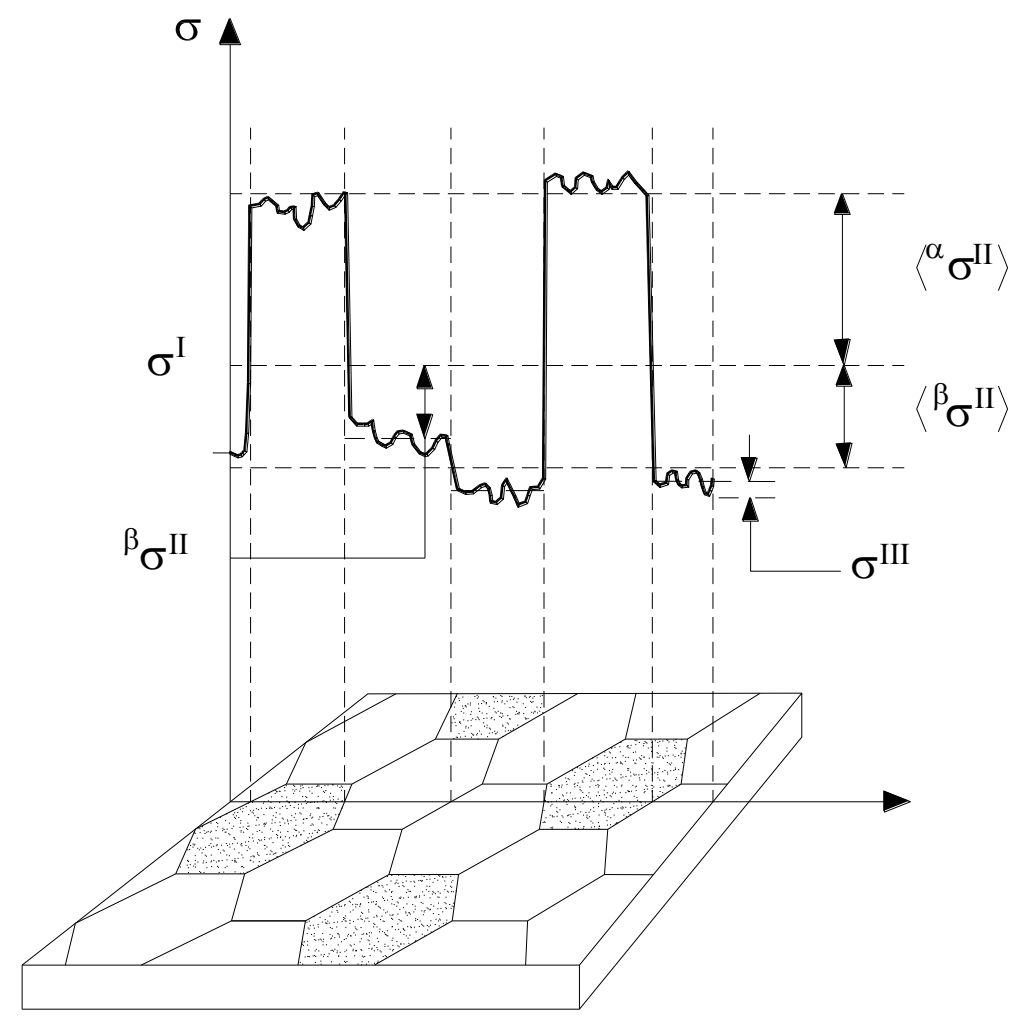

Figure 2-3 The type of residual stresses and the characteristic length scale over which they self-equilibrate. The type $\mathrm{I}\left(\sigma_{\mathrm{rs}}{ }^{\mathrm{I}}\right)$ self-equilibrates over the dimensions of the component, the type II ( $\left.\sigma_{\mathrm{rs}}^{\mathrm{II}}\right)$ self-equilibrates over a few grains ( $\alpha$ and $\beta$ phases) and the type III $\left(\sigma_{\mathrm{rs}}^{\mathrm{III}}\right)$ self-equilibrates within the grain size. (Reprinted from [28]).

Tensile residual stresses are undesirable because of their negative impact on the service life of the components. If the engineering component has an undetected high level of tensile residual stresses then unforeseen failures may occur without any warning [27, 29]. As a consequence, several mechanical and thermal treatments have been developed to control the residual stresses during the manufacturing of the component. For instance, annealing processes are routinely applied after a fusion welding process to reduce the magnitude of tensile residual stresses developed at the weld beam due to the shrinking 
and quenching processes [30]. On the contrary, the introduction of compressive residual stresses on the surface of an engineering component is a common practice to increase the component's service life $[27,29,31,32]$. Thus, a large number of technologies have emerged over the last decades to introduce compressive residual stresses on the surface of engineering components in order to improve their fatigue resistance.

Residual stresses in cracked components have a large influence on the FCG rates that are a function of the alternating stress amplitude and the mean stress [4, 28]. Tensile residual stresses contribute to the increase in the FCG rate, while compressive residual stresses contribute to stopping or delaying the FCG process [33, 34]. For instance, fusion welding processes introduce tensile residual stresses in the weld beams due to a non-uniform temperature distribution and a quenching process that are deleterious for the fatigue life of engineering components. On the contrary, cold hole expansion processes are routinely employed to introduce compressive residual stresses that enhance the fatigue life of engineering structures.

The next section presents a literature review of experimental methods developed for the study of the FCG phenomenon in RSF, which occur due to welding, quenching, cold-hole expansion and overloading.

\subsection{Experimental methods for the study of the fatigue crack growth in residual stress fields}

RSF superimpose over the external load stress fields. In the case of the FCG tests under a constant amplitude stress, the RSF modify the applied stresses at the local domain of the 
crack tip. For instance, Beghini et al. [35] performed experimental FCG tests on the compact tension (CT) specimens obtained from the base metal and weld metal zones of a C-Mn microalloyed steel welded plate. A constant stress ratio $(R)$ of 0.1 was employed, where $R$ is defined as the minimum to the maximum applied stress ratio. Figure 2-4 presents a sketch of the CT specimen cut from the welded plate and the experimental results demonstrating the FCG rate as a function of the stress intensity factor range [35]. The results indicated lower FCG rates for the welded metal zone than for the base metal zone, especially for the initial part of the fatigue tests (at low $\Delta K$ ). At the end of the fatigue test, the FCG rates converge towards a unique value determined by $\Delta K$. Beghini et al. [35] employed a constant stress ratio of 0.1 as previously mentioned. They indicated that the lower FCG rates observed in the welded metal were due to the residual stresses that modify the stress field at the local domain of the crack.

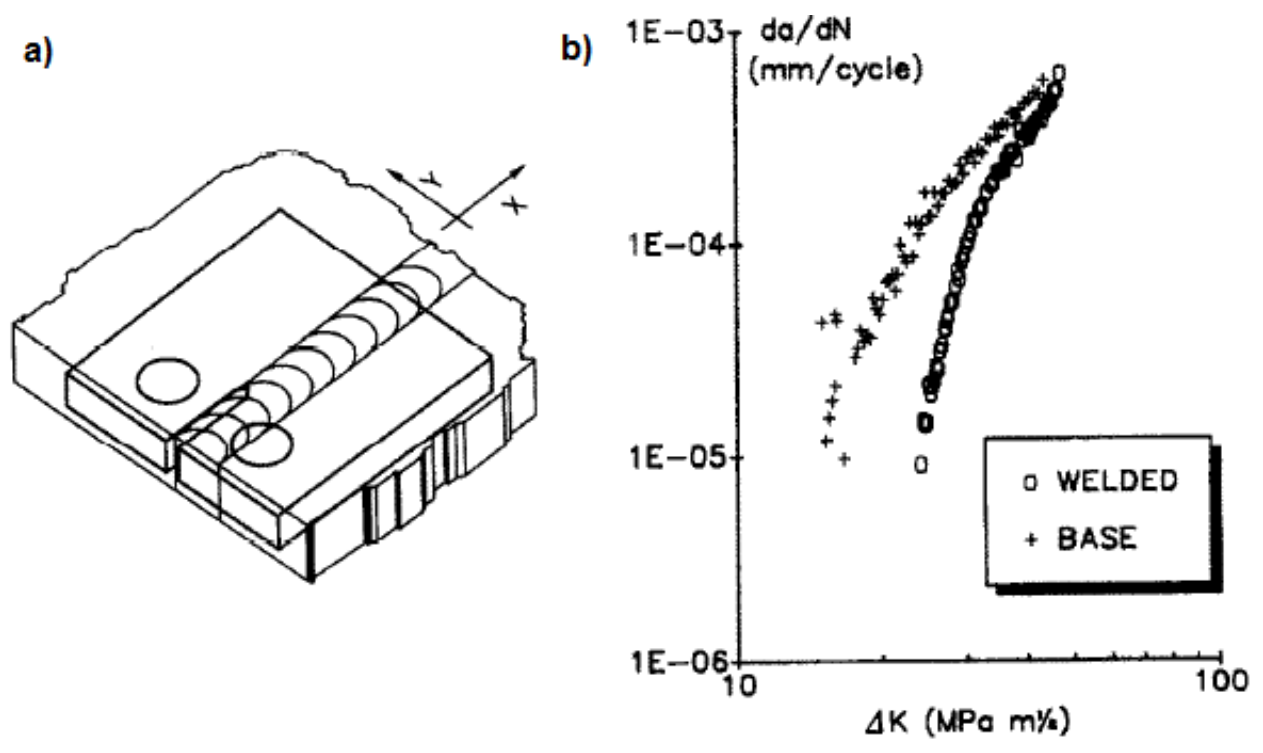

Figure 2-4 a) CT specimen extracted from the welded plate, and b) the fatigue crack growth rate for base and weld metals. (Reprinted from [35], with permission from Elsevier) 
Furthermore, Beghini et al. [35] measured the Back Face Strain (BFS) in the CT specimens during the FCG test at a particular crack length, without reporting it. BFS is the strain on the specimen's side opposite to the crack front. The results indicated a change in the slope of the BFS curve as a function of the applied load for the welded metal zone (Figure 2-5a), while there were no changes in the slope of the BFS as a function of the applied load for the base metal zone [35]. In addition, Beghini et al. [35] reported the presence of a pre-existing residual stress in a CT welded specimen, which was determined by a stress relaxation method [36]. Figure 2-5b presents the results of the study for the residual stress ( $\sigma$ res $)$, the nominal external stress at minimum applied load $(\sigma$ ext $)$ and their sum $(\sigma$ sum $)$ for the CT welded metal specimen [35]. The lower FCG rates for the welded metal zone in relation to the base metal zone (Figure 2-4b) were attributed to the compressive residual stresses at the crack growth plane in the welded metal zone (Figure 2-5b) [35].
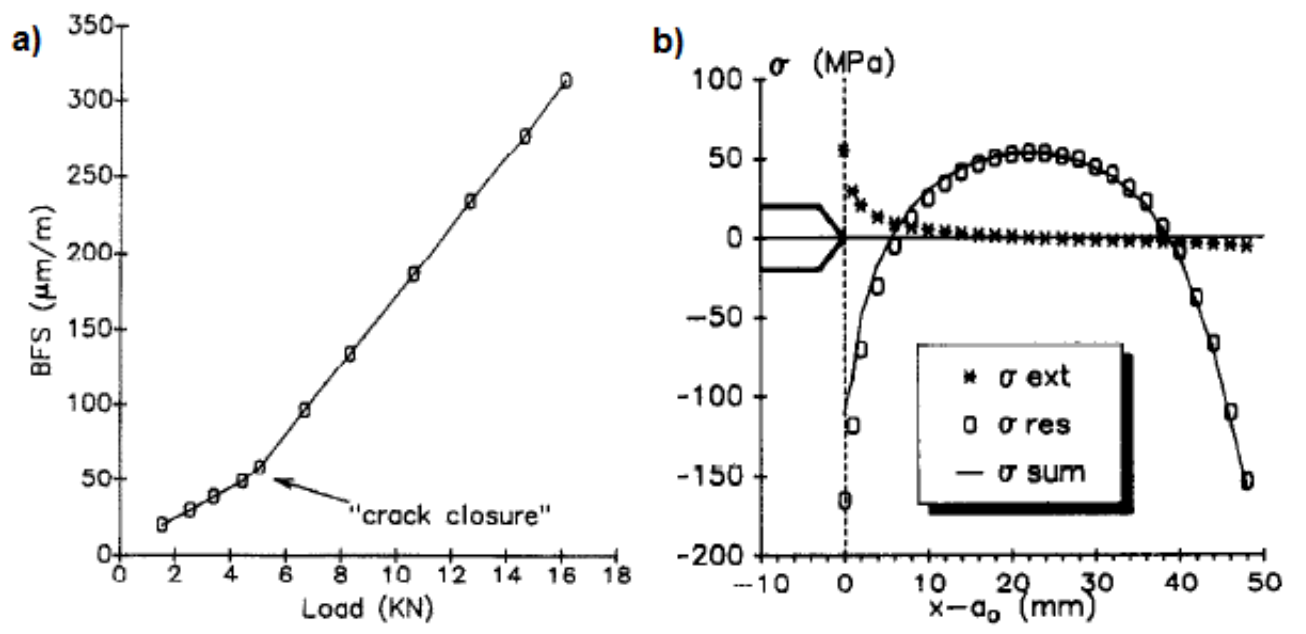

Figure 2-5 a) The back face strain versus the applied load for CT specimen (weld metal) and b) profiles of residual stresses, stresses due to minimum external load and their sum. (Reprinted from [35], with permission from Elsevier). 
The change of the slope of the BFS curve as a function of the applied load is related to the crack closure effect, as first reported by Elber [37]. Elber relates a crack-closure mechanism to the plastic deformation left in the wake (plastic wake) of the FCG. As observed in Figure 2-5a, the crack closure effect is responsible for a change in the compliance of the specimen during the applied cyclic load. For the FCG tests at positive or zero stress ratios, the crack closure effect may induce a contact between the crack faces (crack closure) under tensile applied loads with a low magnitude. Several authors have confirmed the crack closure effect due to plastic wake $[38,39,40,6]$. As a result of the crack-closure mechanism, the nominal applied stress-intensity factor range $\Delta K$ in the fatigue crack grow test does not correspond to the actual difference between the maximum and minimum stress intensity factors so that $\Delta K$ is not fully effective and depends on the crack opening stress [37]. Beside the crack-closure mechanism that is related to the plastic wake, other mechanisms of the crack closure have been reported such as roughness, oxides and metallurgical transformations [39].

Fitzpatrick et al. [6] analyzed the FCG in RSF due to a quench process in a metal matrix composite of $2124 \mathrm{Al}$ reinforced with 20 weight percent of $\mathrm{SiC}$ particles of $3 \mu \mathrm{m}$ nominal diameter. They used the neutron diffraction technique to measure the RSF in the Al matrix for naturally aged (NA) and artificially over-aged (OA) samples. Figure 2-6a presents the residual strain results in the Al matrix for both material conditions. The naturally aged sample presents a large level of residual strains in comparison to the over aged sample due to finely dispersed coherent precipitates with disc and plate shapes [41]. The surface of the metal-matrix composite sample with naturally aged condition 
presented compressive residual strains, which induced a parabolic residual stress field as observed in Figure 2-6a [6]. Diffraction techniques are only sensitive to elastic strains, because they employ the lattice parameter of the crystalline structure as the gauge to measure the strains. Thus, the residual strains in Figure 2-6a correspond to the residual stress field. Compliance measurements of a cracked NA sample in a four-point-bending test indicated a crack closure effect that was characterized by a gradual change in the slope of the BFS curve as a function of the applied load (Figure 2-6b). The nominal applied load that induces the crack faces to open is employed to define an opening stress intensity factor $\left(K_{o p}\right)$. Three definitions could be formulated for the opening stress intensity factor: $K_{c l, l o}, K_{c l, m i d}$ and $K_{c l, u p}$ as shown in Figure 2-6b. The change in the initial and final slopes of the compliance curve defines the lower and upper values for the opening stress intensity factor $\left(K_{c l, l o}\right.$ and $\left.K_{c l, u p}\right)$, and the intersection of both slopes defines a mid-value $\left(K_{c l, \text { mid }}\right)$ [6]. The experimental measurements of the crack closure effect are difficult to obtain due to a large number of underlying mechanisms, i.e. roughness or plastic wake. Besides, the mechanical compliance curve is dependent on the measurement system used to monitor the displacement. For instance, the choice of a sensor (strain gauge, extensometer, etc.) provides different compliance curves [9] and there are different opinions about the best method [42]. Numerical models have also been used to calculate the crack opening stress $[43,44,45]$ as explained in the following section. 

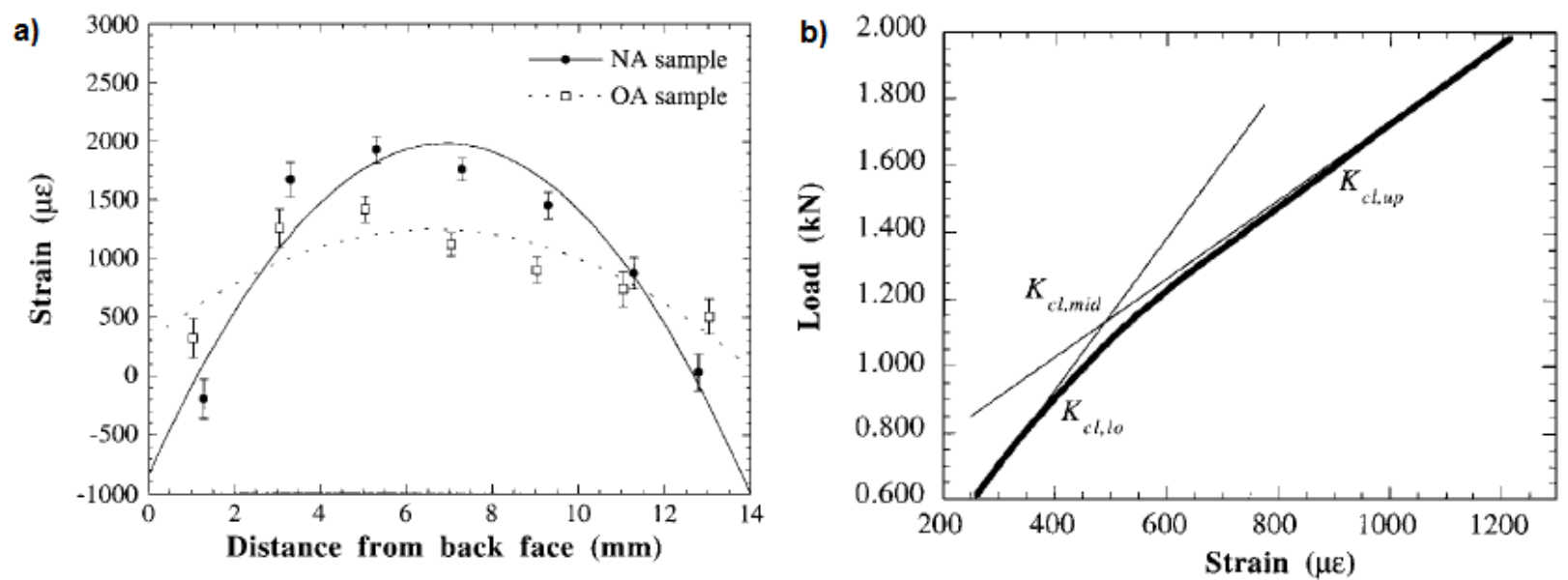

Figure 2-6 a) Residual strain measurements for a natural aged (NA) and an over aged (OA) Al matrix composite, and b) load vs back face strain during the four-point-bending of Al-matrix specimen with NA condition. (Reprinted from [6] with kind permission from Springer Science and Business Media. Copyright 1998 ASM International).

Figure 2-7 presents the crack opening stress intensity factor values obtained during the FCG test according to the three definitions for the opening load $\left(K_{c l, ~ l o}, K_{c l, \text { mid }}\right.$ and $\left.K_{c l, u p}\right)$. The results are shown for the naturally aged (NA) and the over aged (OA) samples [6]. Both conditions, the NA and the OA, exhibited a crack closure effect, but the NA showed higher variations in the crack closure as a function of the crack length than the OA condition due to the expected higher levels of RSF (Figure 2-6a). Besides, the NA material exhibited a large level of crack closure variations. However, through the application of a curve fit to the three closure level variations, it was observed that they all converged at a point at the initial crack length of $\sim 5 \mathrm{~mm}$, thus indicating a well-defined crack opening stress. 

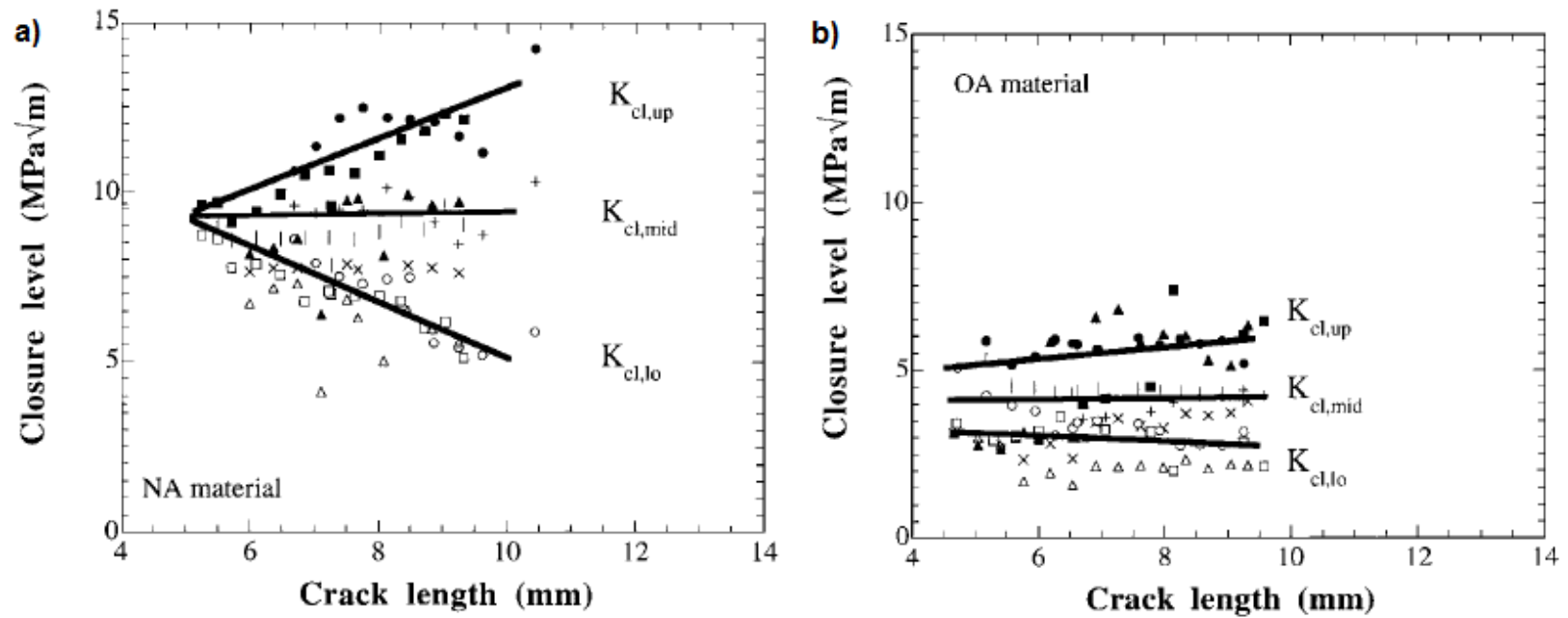

Figure 2-7 Crack closure level as a function of the crack length for: a) the natural aged (NA) condition and b) the over aged (OA) condition. (Reprinted from [6], with kind permission from Springer Science and Business Media).

Lacarac et al [46] analyzed the FCG in cold-hole expanded specimens using experimental and numerical methods. The former are presented in this section and the latter are reviewed in the next section. The cold-hole expansion process introduces an annular region of compressive residual stresses in the component and it has been widely employed in rivet holes on the skin of aircraft structures to extend their fatigue life [4]. The cold-hole expanded specimens in Lacarac et al.'s study [46] were made of a commercial age-hardened aluminum alloy. The dimensions of the rectangular specimens were $245 \times 100 \times 6 \mathrm{~mm}$ with a drilled $6.35 \mathrm{~mm}$ diameter hole at the center of the specimen. The specimens were subject to a radial expansion equivalent to the $4 \%$ strain, after which an initial crack was machined on each side of the hole. Figure 2-8a presents a schematic of a corner crack with a radius of $0.25 \mathrm{~mm}$ emanating from the cold hole expanded specimens [46]. The yield strength of the aluminum alloy determined from an 
experimental uniaxial tensile test was $426 \mathrm{MPa}$ according to the $0.1 \%$ proof stress [46]. FCG tests of cold-hole expanded specimens were conducted at constant stress ratios of 0.1 and 0.7 with the maximum applied nominal stress of 0.38 times the yield strength of the aluminum alloy. In addition, a FCG test of a hole-specimen without the expansion process was conducted to characterize the base material behavior (free of residual stresses) at a constant stress ratio of 0.7 (Figure 2-8b). In the case of the cold-hole expanded specimens the results of the FCG rate as a function of the crack length are presented in Figure 2-8 $\mathrm{c}$ and d [46]. The plots show the experimental results (open symbols and continuous line) along with the numerical predictions (closed symbols). The methodology employed for the numerical results is explained in the next section. According to the experimental results, the FCG rates decreased during the initial part of the fatigue test, i.e., from the initial crack length to a crack length of $\sim 2 \mathrm{~mm}$. For the remained part of the fatigue test, the FCG rate increased. Lacarac et al. pointed out that at around $2 \mathrm{~mm}$, the pre-existing residual stresses due to the cold-hole expansion shifted from a compressive to tensile state and related this shift to the increase in the FCG rate. Lacarac et. al also conducted a numerical analysis to predict the FCG rates which is described in the next section of this manuscript along with other models from the literature. 
a)

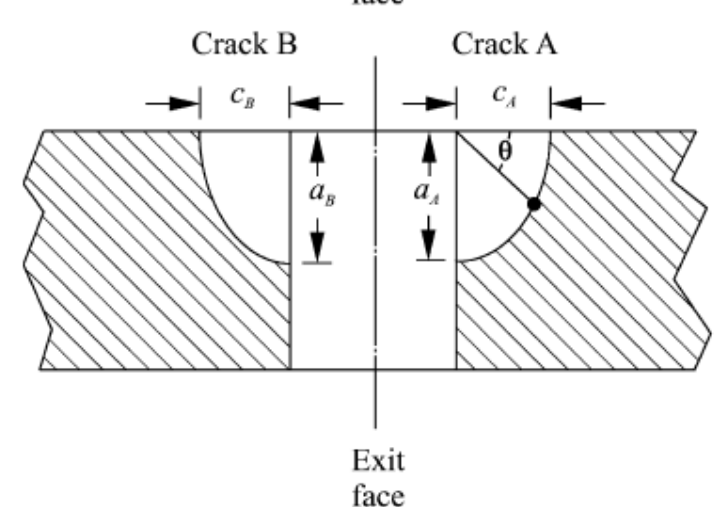

b)

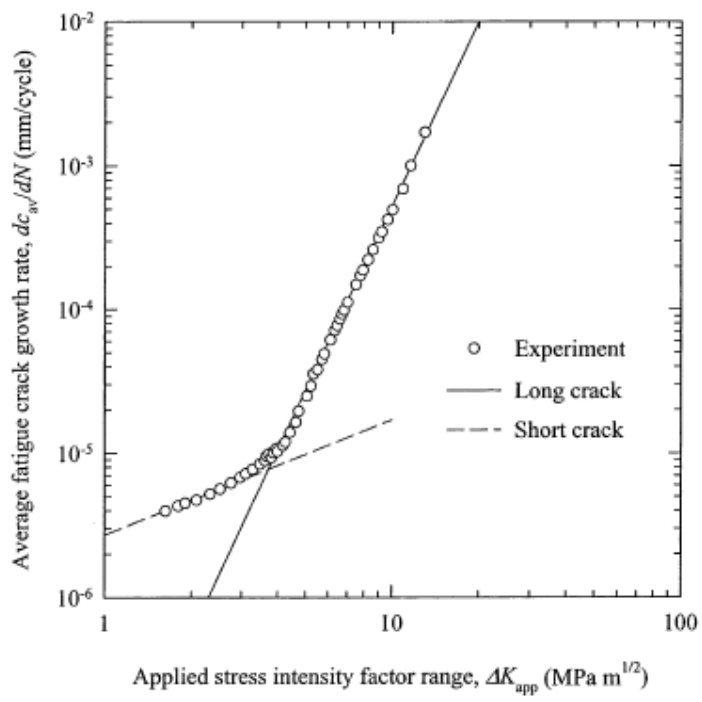

c)

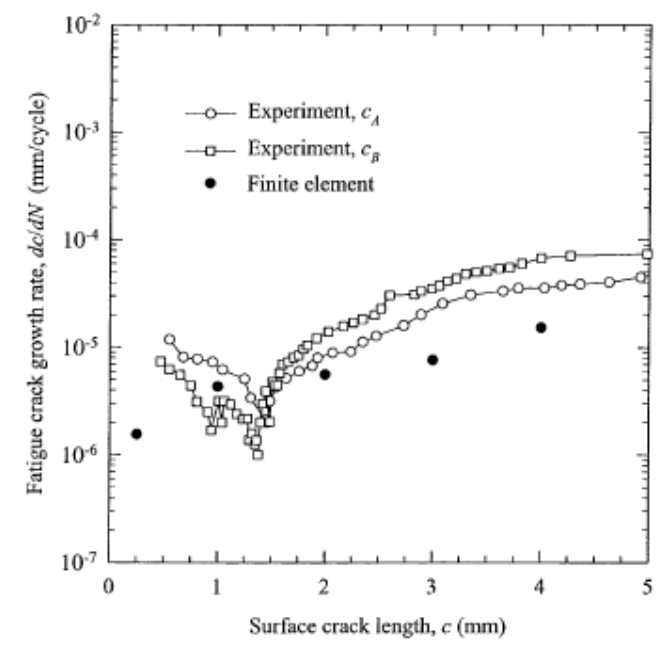

d)

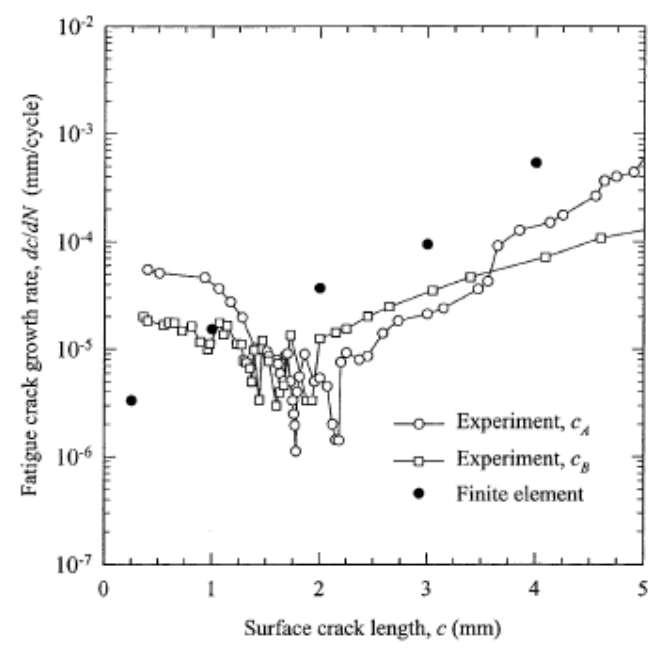

Figure 2-8 a) Details of initial cracks machined in cold-hole expanded specimens; b) the fatigue crack growth behavior for a non-cold expanded hole with the Paris-Erdogan relationship fit for short and long cracks, and the fatigue crack growth rate as a function of the crack length for: b) $R=0.7$ and $\sigma_{\max }=162 \mathrm{MPa}$ and c) $R=0.1$ and $\sigma_{\max }=162$ MPa. (Reprinted from [46], with permission from Elsevier).

Lee et al. [47] analyze the evolution (redistribution and relaxation) of RSF produced by a single tensile overload during a FCG test. Figure 2-9a presents the crack length $(a)$ versus 
the number of applied cycles $(N)$, along with a sketch of the single tensile overload applied during the FCG test at 20,000 load cycles.

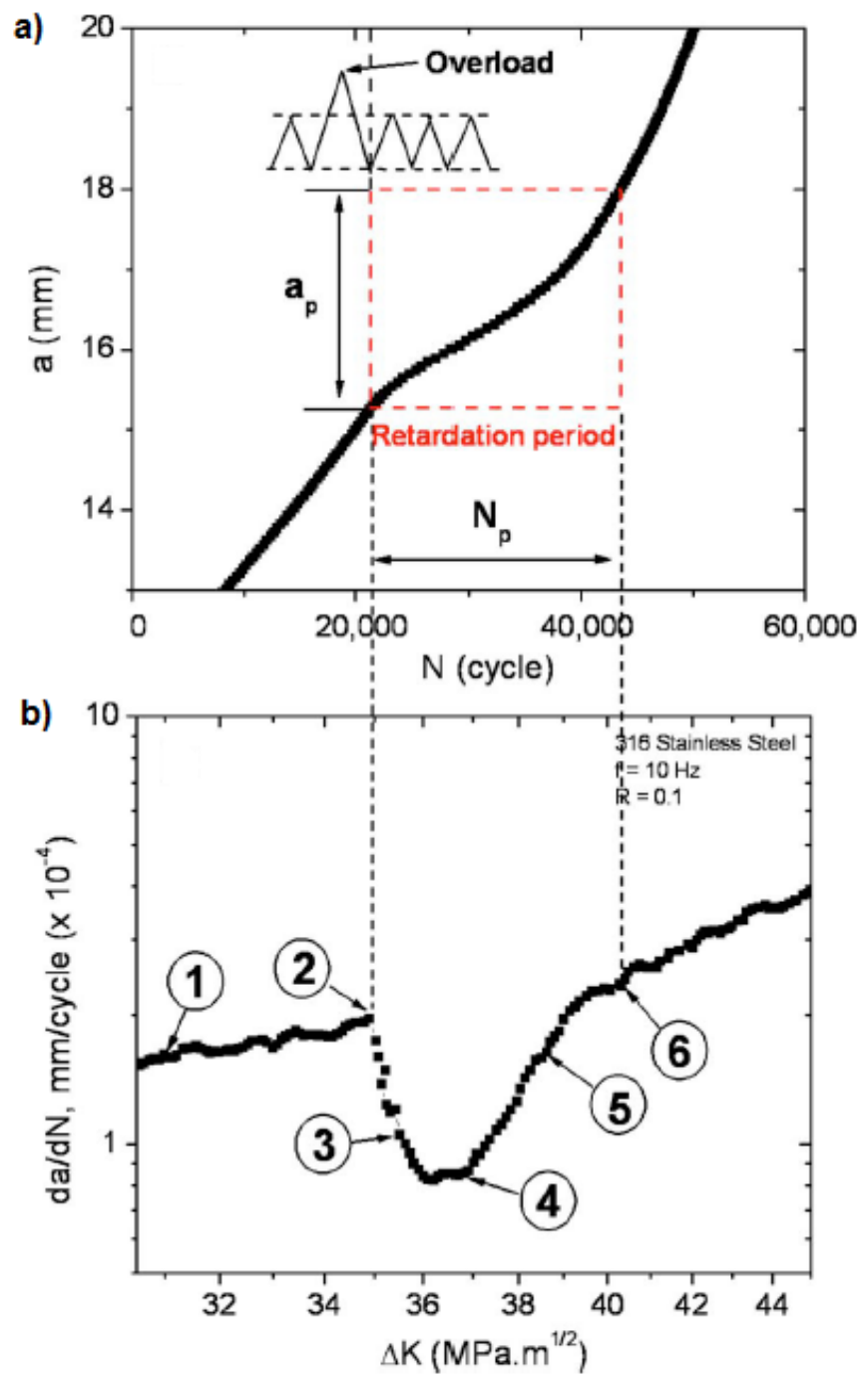

Figure 2-9 Fatigue crack growth results for a single tensile overload: a) The crack length $a$ vs the number of cycles $N$, and b) the fatigue crack growth rate $d a / d N$ vs the stressintensity factor range $\Delta K$. Six specimens were prepared at different crack-growth stages to characterize the residual stresses by the neutron diffraction technique. (Reprinted with permission from [47]. Copyright 2010, AIP Publishing LLC.) 
FCG tests of the CT specimens were conducted under a constant stress ratio $(R)$ of 0.1 . Six CT specimens were subjected to a different number of applied cycles to characterize the evolution of RSF during the FCG test, i.e., before, at and after a single tensile overload. The RSF were measured with a neutron diffraction technique. The CT specimens were made of a low-carbon austenitic stainless steel enriched with nitrogen (AISI 316LN). Figure 2-9b presents the FCG rate as a function of the stress intensity factor range, where the numbers ( 1 up 6) indicate the position for the residual stress characterization with respect to the FCG stage in each CT specimen. The CT specimen 1 (SP1) corresponded to a FCG stage previous to the application of the tensile overload; the CT specimen 2 (SP2) corresponded to the FCG stage at the overloading point; the CT specimens 3, 4 and 5 (SP3, SP4 and SP5) corresponded to the FCG stage during the retardation period; and the CT specimen 6 (SP6) corresponded to the FCG stage at the end of the retardation period. The FCG rates (Figure 2-9b) indicated that the single tensile overload induced a crack growth retardation period.

Figure 2-10 presents the residual stresses determined by the neutron diffraction technique at the FCG plane as a function of the distance from the overload point for the CT specimens SP1, SP2, SP4 and SP6. In Figure 2-10a, both SP1 and SP2 presented compressive residual stresses in front and behind the crack tip, but SP2 presented a large zone of compressive residual stresses in front of the crack tip with a higher magnitude $(\sim 250 \mathrm{MPa})$ due to the overload. Behind the crack tip, the compressive residual stresses seemed to follow a trend towards the zero magnitude (complete relaxation) in SP2. 

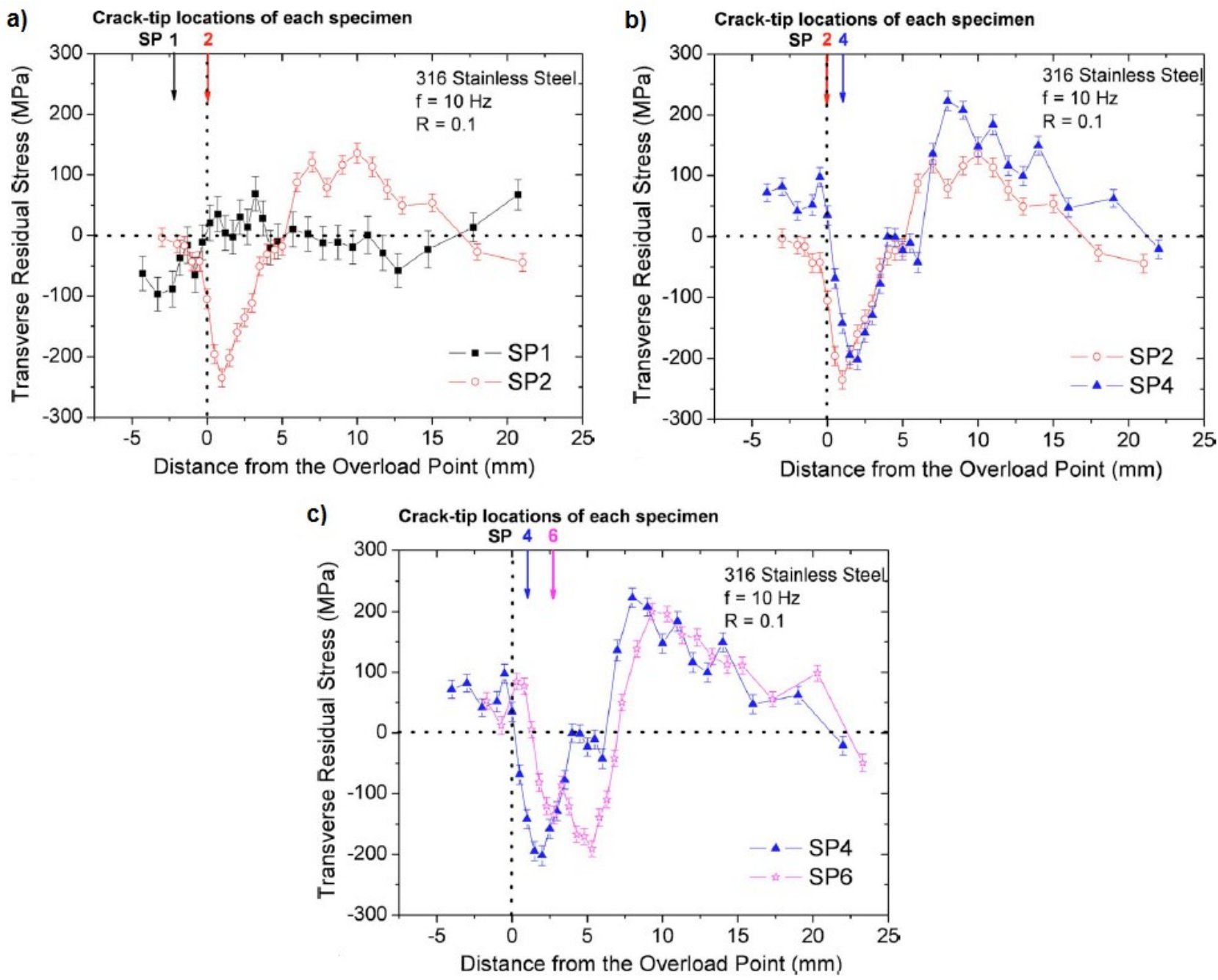

Figure 2-10 Residual stresses as a function of the distance from the overload point for specimens: a) SP1 \& SP2, b) SP2 \& SP4, and c) SP4 \& SP6. (Reprinted with permission from [47]. Copyright 2010, AIP Publishing LLC.)

After the single tensile overload, the FCG rate decreased gradually rather than abruptly towards a minimum FCG rate as observed in Figure 2-9b. This gradual decrease in the FCG rate due to a single tensile overload that introduces compressive residual stresses at the crack tip had been previously reported by other authors in the literature [48] and it was in concordance with the previously reported FCG rate behavior due to residual 
stresses observed in Figure 2-8c and d [46]. In the case of SP4 and SP6 specimens corresponding to the FCG stage post-overload, the residual stress field showed redistribution with respect to SP2. In general, the compressive residual stresses were relaxed, while the tensile residual stresses increased their magnitudes as observed in Figure $2-10 \mathrm{~b}$ and $\mathrm{c}$.

\subsection{Numerical models for fatigue crack growth in residual stress fields}

Several commercially-available computer programs have been developed for fracture mechanics and FCG analyses. AFGROW [49] and NASGRO [50] are two examples of these programs, which are employed to predict FCG rates. Also, they can be employed to

predict fatigue crack initiation based on the stress- and strain- life curves. Some additional capabilities and features of the programs are the spectrum filter and communication interface. The spectrum filter allows the user to employ different solutions for tension and compression load spectrums, while the communication interface allows the user to execute an analysis via another computer application. In general, AFGROW and NASGRO work with libraries that contain a large amount of experimental fatigue data and closed form solutions of the stress intensity factor $(K)$ for different geometries $[51,52]$. The users perform a FCG analysis for a component by defining its load history, geometry and selecting the combination of the fatigue data (material properties) and the closed form solution of $K$ that best suits the problem. Forman et al. [53] published a relationship between the FCG rate and the stress intensity factor range known as the NASGRO equation. It employs the FCG threshold $\Delta K_{t h}$, the fracture 
toughness $K_{c}$ and several fitting parameters to characterize the three regions of the FCG behavior ( $d a / d N$ vs $\Delta K$ curves). Along with the NASGRO equation other relationships are employed by AFGROW and NASGRO to predict the FCG process, e.g., the Walker equation [54]. NASGRO and AFGROW have been successively used to predict FCG phenomena. However, neither the NASGRO nor AFGROW program deals with the analysis of FCG in components with pre-existing residual stresses $[49,50]$.

The numerical models employed in the literature to predict the FCG in RSF have concentrated on elastic superposition and effective stress-intensity factor methods. The elastic superposition method is valid for small-scale yielding conditions, which is the case if the cyclic plastic zone is considerably smaller than any dimension of the component so that a stress-intensity factor $K$ completely characterizes the stress, strain and displacement fields around the crack tip. The elastic superposition method consists of adding the stress-intensity factors due to the applied load $K^{S}$ and the pre-existing residual stresses $K^{R S}$ to compute a total stress intensity factor:

$$
K^{T}=K^{S}+K^{R S}
$$

A total stress intensity factor range and a total stress ratio are determined at the maximum and minimum applied loads during the FCG process, according to:

$$
\begin{gathered}
\Delta K_{T}=K_{\max }^{T}-K_{\min }^{T} \\
R^{T}=\frac{K_{\min }^{T}}{K_{\max }^{T}}
\end{gathered}
$$

An empirical FCG relationship is employed to predict the FCG rate $\frac{d a}{d N}$ :

$$
\frac{d a}{d N}=f\left(\Delta K^{T}, R^{T}\right)
$$


Numerical integration is used to compute a number of cycles $(N)$ required for the crack to grow from an initial crack length $a_{i}$ to a final crack length $a_{f}$ :

$$
N=\int_{a_{i}}^{a_{f}}\left(\frac{d N}{d a}\right) d a
$$

The elastic superposition method has been criticized in the literature $[7,55,56]$ because it only considers the original - pre-existing - residual stresses of the uncracked component and does not consider the evolution of residual stresses - relaxation and redistribution during the FCG - observed by experimental studies. For instance, the data shown in Figure 2-10 demonstrate the relaxation of the residual stresses behind the crack tip for specimen SP2 and a complete redistribution of the residual stresses for specimens SP4 and SP6. An intense debate is found in the literature between supporters of the elastic superposition method $[32,57]$ and those who question its applicability for the prediction of FCG in RSF $[5,6,7]$. Moreover, other authors agree with the use of the elastic superposition method if the crack closure phenomenon is considered [58, 59]. In the case of the specimens subjected to thermal or mechanical processes to relieve the RSF, the correlation between the stress intensity factor range and the FCG rate according to an empirical relationship has been successfully employed to predict FCG [4]. However, for the specimens subjected to beneficial compressive residual stresses, the elastic superposition method has failed to provide reliable predictions [60, 61, 62, 63, 64, 65]. Jones and Dunn $[59,56]$ employed the Finite Element Analysis (FEA) method to compute the total stress intensity factors and analyze the FCG in RSF according to the elastic superposition method. The specimens analyzed were the Single-Edge Bend (SE(B)) [59] and cold-hole expanded [56], and the material considered was a 2024-T351 aluminum alloy. The redistribution of RSF was not considered in the analysis and the 
pre-existing residual stresses in the uncracked specimens were employed as crack face pressures in the FEA models in order to compute $K^{R S}[59,56]$. Figure 2-11 presents the pre-existing residual stresses for $\mathrm{SE}(\mathrm{B})$ [59] and cold-hole expanded specimens [56].
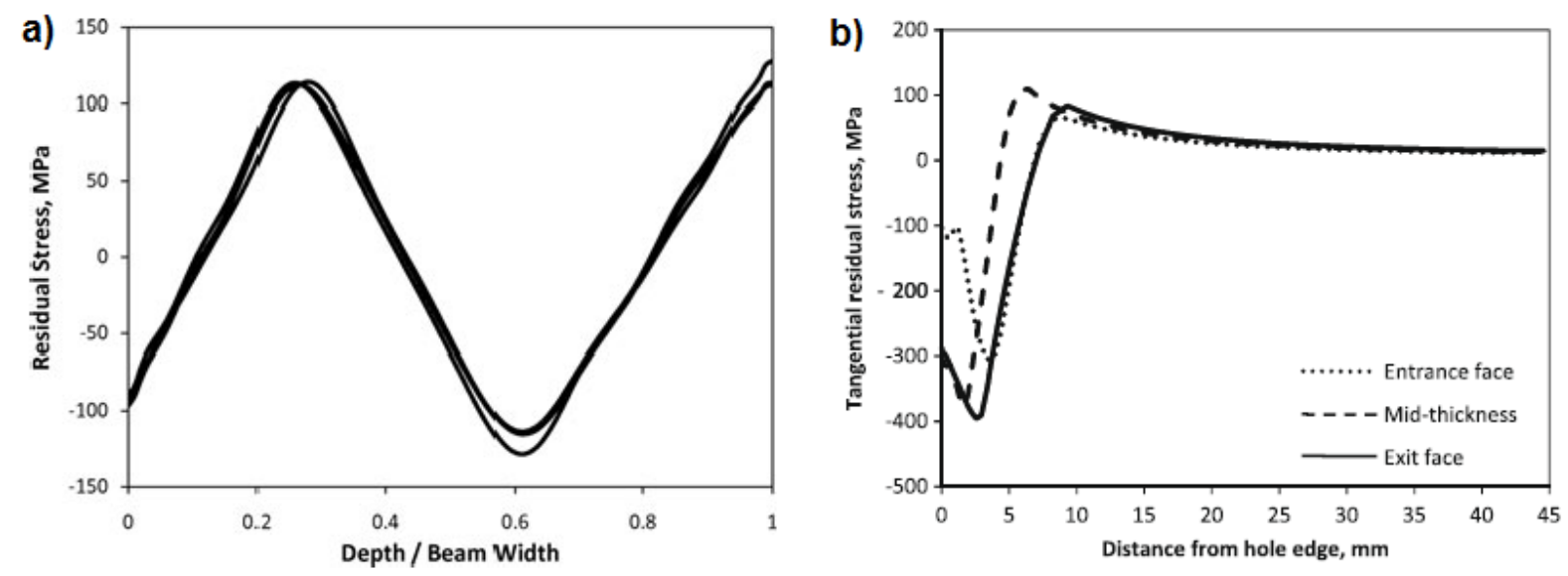

Figure 2-11 Pre-existing residual stresses in an uncracked specimen: a) Single-Edge Bend $\mathrm{SE}(\mathrm{B})$, and b) cold-hole expanded. (a) Reprinted from [59], with permission from John Wiley and Sons; Copyright 2008 Blackwell Publishing Ltd. (b) Reprinted from [56] with permission from Elsevier; Copyright 2009 Elsevier.

In the case of the $\mathrm{SE}(\mathrm{B})$ specimen, an experimental FCG test with a constant stress ratio ( $R=0.1$ ) was conducted to verify the numerical results of the elastic superposition method [59]. Further, the FCG test was conducted under a constant stress intensity factor range by dropping the applied load as a function of the crack length. The crack was introduced into the specimen's side with compressive residual stresses. Figure 2-12 presents predictions and measurements of the FCG rate and the fatigue life for the $\mathrm{SE}(\mathrm{B})$ specimen with and without residual stresses [59]. 
a)

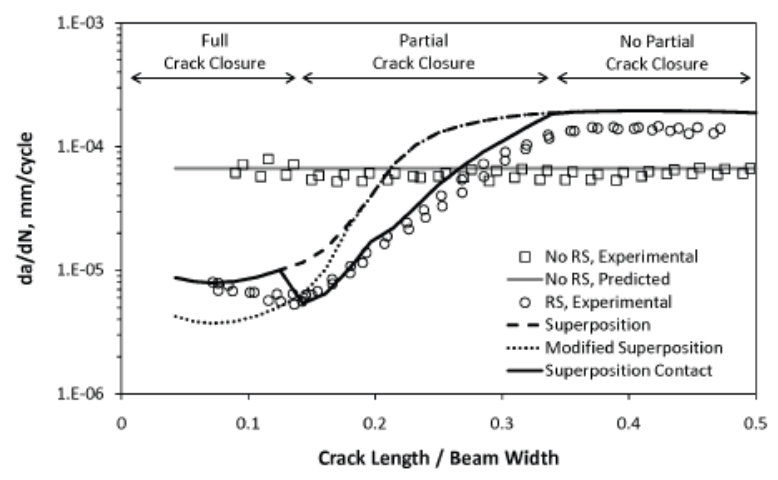

b)

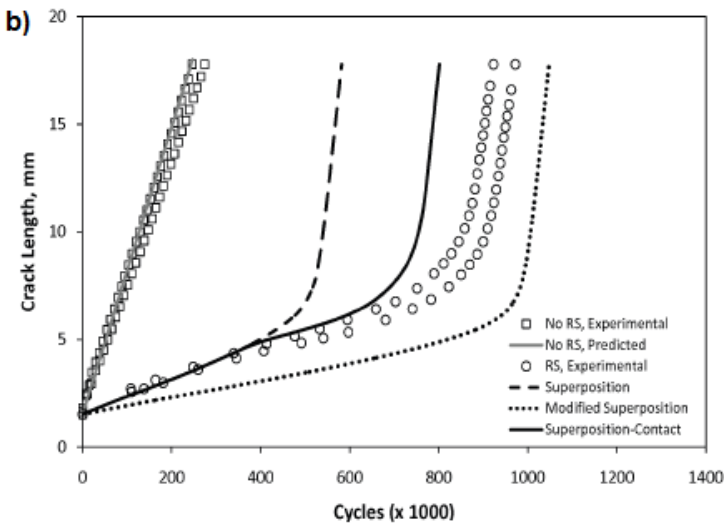

Figure 2-12 Predictions and measurements of: a) the fatigue crack growth rate, and b) the fatigue life. (Reprinted from [59], with permission from John Wiley and Sons; Copyright 2008 Blackwell Publishing Ltd.)

FCG rates were constant for the SE(B) without residual stresses because the applied load was decreased during the FCG test. The superposition method over-predicted the FCG rates in the $\mathrm{SE}(\mathrm{B})$ specimen with residual stresses and under-predicted the fatigue life (Figure 2-12). In addition to this superposition method previously described by equations 1 through 4, modified superposition and superposition contact versions were employed by [59]. The modified version was equivalent to the original version (elastic superposition method) if $K_{\min }^{T}$ was positive, but if $K_{\min }^{T}$ was negative then it was replaced by zero:

$$
\begin{gathered}
\Delta K^{T}=K_{\max }^{T}=K_{\max }+K^{R S} \\
R^{T}=0
\end{gathered}
$$

The modified superposition method under-predicted the FCG rates when $K_{\min }^{T}$ was negative, while for positive $K_{\min }^{T}$ the modified superposition over-predicted the FCG rates (Figure 2-12a). The fatigue life was over predicted by the modified superposition 
model (Figure 2-12b). In the case of the superposition contact version, the crack closure effect was incorporated into the FEA analysis by setting a contact interaction at the crack face. If the crack was completely closed at the minimum applied load then the superposition contact version was equivalent to the original version (elastic superposition method), but if the crack was partially closed at a minimum applied load then a new $K_{\min }^{T}$ was defined from the FEA analysis according to the minimum applied load that overpassed the crack closure effect. As can be observed in Figure 2-12, the predictions of the FCG rate when the crack was partially closed were in a good agreement with the experimental measurements, but when the crack was completely closed or opened, the superposition contact method over predicted the FCG rates. The fatigue life was underpredicted by the superposition contact version, but the prediction was better than the corresponding one from the elastic superposition version without the crack closure effect. Nevertheless, the FCG rates under the compressive residual stresses in the $\mathrm{SE}(\mathrm{B})$ were either over- or under-predicted by the three superposition versions. In the case of the cold-hole expanded specimen, Jones and Dunn [56] only employed the elastic superposition method to analyze the FCG in RSF. They reported measurements and predictions of the fatigue life (Figure 2-13) for the cold-hole expanded specimen, but the results for the FCG rate were not reported [56]. The results indicated that the elastic superposition method over predicted the fatigue life by a significant amount, which means it was not able to provide reliable predictions. 


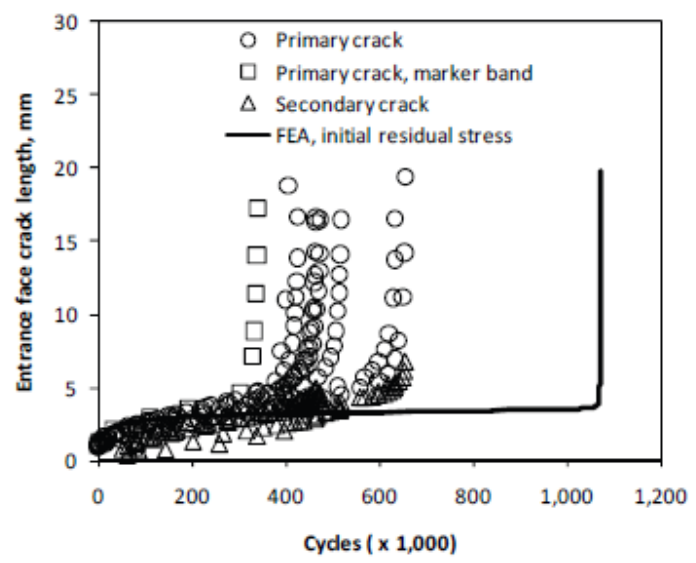

Figure 2-13 Predictions and measurements of fatigue life for cold-hole expanded specimen. (Reprinted from [56] with permission from Elsevier; Copyright 2009 Elsevier.)

The elastic superposition method with the incorporation of the crack closure effect was employed by Lacarac et al. [46] in order to predict the FCG in RSF in cold-hole expansion processes. A general description of the numerical analysis was already presented in the previous section of this manuscript. Lacarac et al. employed an axisymmetric finite element model to simulate the cold-hole expansion process and predict the RSF. Large regions of compressive residual stresses were resolved by the axisymmetric finite element model at the edge of the hole, which were dependent on the thickness of the specimen. Pre-existing residual stresses from the cold-hole expansion simulation were prescribed to a set of three-dimensional finite element models of a rectangular specimen with a corner crack at the edge of a hole. In total, 5 corner crack configurations with different diameters $(0.25,1,2,3$ and $4 \mathrm{~mm})$ were analyzed. In addition, a set of nominal tensile loads was applied to the finite element models of the rectangular specimen to compute the stress intensity factor. Figure 2-14a presents the 
stress intensity factor $(K)$ as a function of the applied stresses for a corner crack with 2 mm diameter.
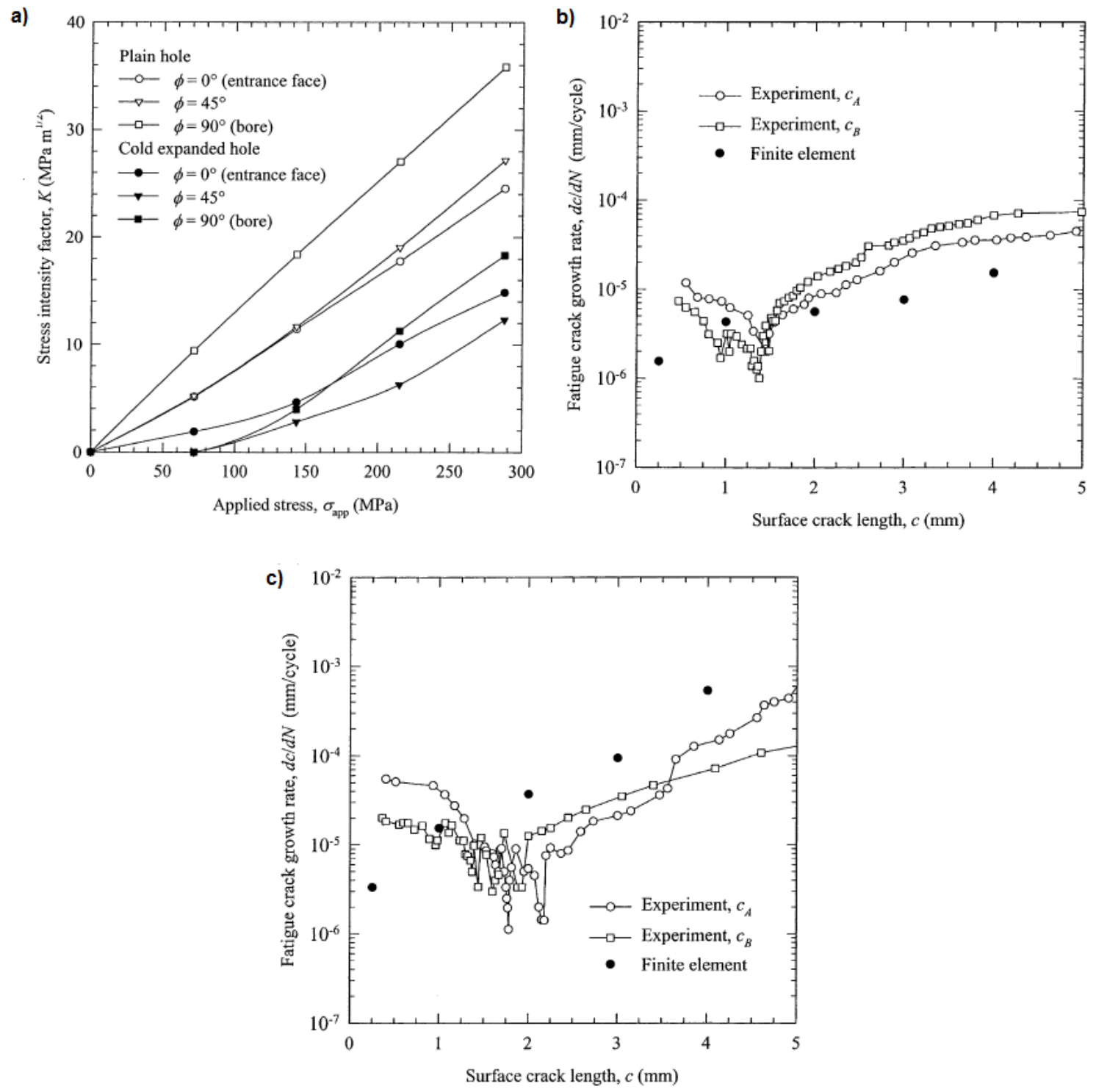

Figure 2-14 a) The stress intensity factor as a function of the applied stress for plain and cold expanded holes with a $2 \mathrm{~mm}$ diameter. The fatigue crack growth rate as a function of the crack length for: c) $R=0.7$ and $\sigma_{\max }=162 \mathrm{MPa}$ and d) $R=0.1$ and $\sigma_{\max }=162 \mathrm{MPa}$. (Reprinted from [46] with permission from Elsevier. Copyright 2003). 
The results in Figure 2-14a showed a non-linear variation of $K$ as a function of the applied load for the cold-hole expanded specimen because of the pre-existing residual stresses. For the angular positions $\left(\phi=45^{\circ}\right.$ and $\left.90^{\circ}\right)$ along the crack front, the results indicated a crack closure effect for applied stresses approximately below $75 \mathrm{MPa}$. The predictions of the FCG rate were already presented in Figure 2-8 b and $\mathrm{c}$ in conjunction with the experimental measurements, but Figure 2-14 b and c presents the FCG rates again.

The predictions of the FCG rate with the elastic superposition and contact conditions for the crack faces roughly agreed with the experimental measurements as observed in Figure $2-14 \mathrm{~b}$ and $\mathrm{c}$. However, the predictions failed to capture the transient behavior observed in the experimental measurements of the FCG rate. Consistent with the conclusions drawn by Jones et al. [56], Lacarac et al. [46] argued that a lack of accuracy in the cold-hole expansion finite element simulation was the reason for a poor agreement between numerical and experimental results. Furthermore, they indicated that the redistribution of residual stresses and the crack closure due to plastic wake were other possible sources of error in their predictions since these effects were not considered by the finite element model [46].

The effective stress intensity factor range is an alternative approach to elastic superposition methods for the prediction of the FCG in RSF. Even under a small scale yielding condition, plastic deformation is induced at the crack tip during the FCG and a plastic wake is left on the path of the growing crack. A direct consequence of the plastic wake is the crack closure phenomena discovered by Elber [37], as previously described. Elber postulated that the FCG rate was a function of an effective stress intensity factor 
range rather than the nominal one. The effective stress intensity factor range was defined as:

$$
\Delta K_{e f f}=K_{\text {max }}-K_{o p}
$$

where $K_{\max }$ is the stress intensity factor at the nominal maximum applied load and $K_{o p}$ is the crack opening stress intensity factor. Experimental methods have been employed to determine $K_{o p}$ as a function of the crack closure effect, as described in the previous section of this chapter. In the cases of the numerical analysis, the FEA method has been employed to simulate plasticity-induced fatigue crack closure and to determine $K_{o p}$. Solanki et al. [45] provides an overview of the technique used to simulate plasticityinduced fatigue crack closure with the application of the FEA method; several other publications also discuss the topic [43, 44, 45]. However, only LaRue and Daniewicz [8] applied the technique for a crack growing through the pre-existing RSF. The plasticityinduced crack closure technique focuses on determining the fraction of the applied stress (crack opening stress) that overcomes the crack closure effect. The effective stress intensity factor range is then computed as the difference between the maximum stress intensity factor and the crack opening stress intensity factor (eq. 8). LaRue et al. [8] set the simulation to first introduce the pre-existing residual stresses, which is followed by a plasticity-induced crack closure analysis where in the crack was incrementally advanced one FEA element length at a time for each cyclic load. During each crack increment, the crack opening stress was defined as the applied stress that first fully opened the crack. A requirement for the plasticity-induced crack closure FEA simulation is that the element size must be smaller than the size of the plastic zone in front of the crack tip, otherwise the plasticity zone is not resolved. LaRue et al. [8] used the element size of $0.2117 \mathrm{~mm}$ 
and conducted the simulation of the FCG from $\sim 2 \mathrm{~mm}$ to $38 \mathrm{~mm}$. The FEA results of the effective stress intensity factor range in conjunction with experimental and NASGRO FCG curves were employed to predict the FCG in RSF. In addition, the elastic superposition method was used to compare the results with respect to the plasticityinduced crack closure method results. Figure 2-15 presents the FEA model mesh, the FEA results of the crack opening stresses as a function of the crack length, the experimental and NASGRO FCG curves and the fatigue life predictions and experimental measurements (Liu Data).

a)

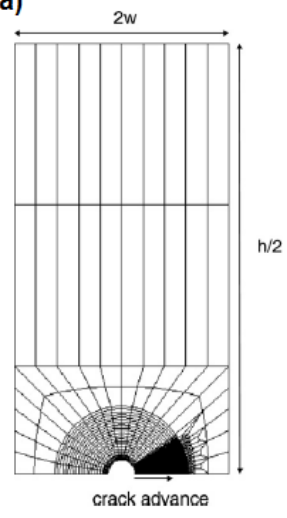

c)

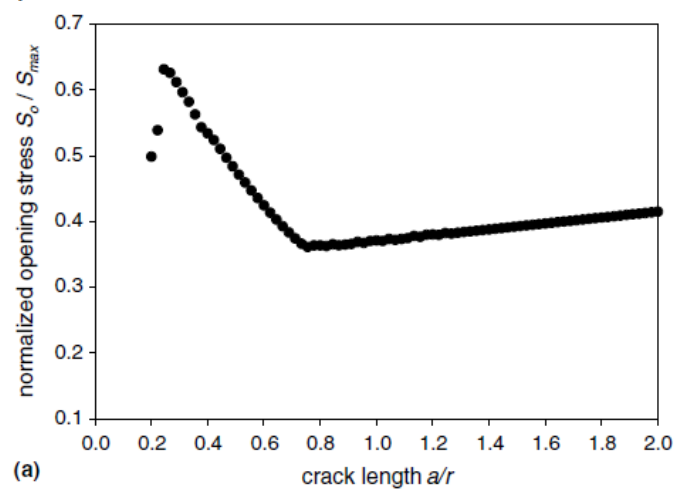

b)

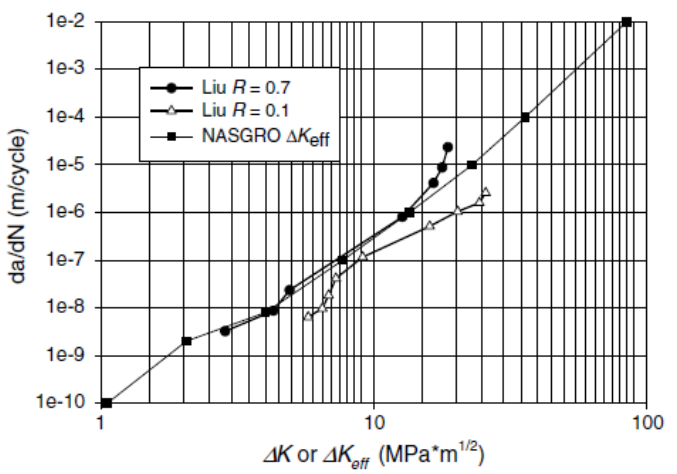

d)

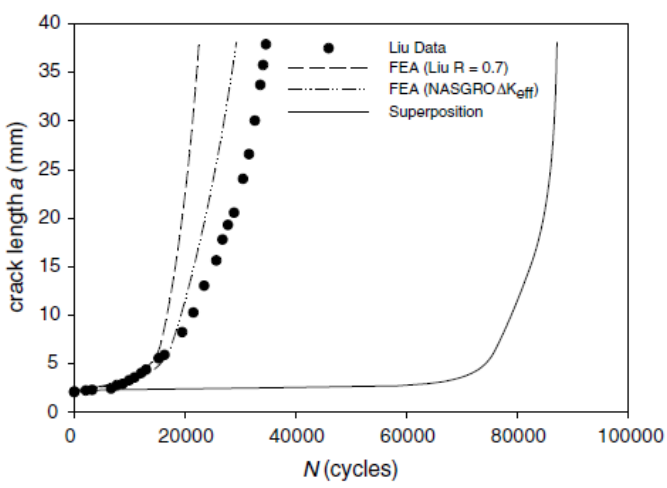

Figure 2-15 a) FEA model mesh, b) FEA results of the crack opening stress as a function of the crack length, c) the experimental and NASGRO fatigue crack growth curves, and d) the predictions of fatigue life. (Reprinted from [8] with permission from Elsevier. Copyright 2006 Elsevier Ltd.) 
LaRue et al. concluded that the plasticity-induced crack closure FEA method resulted in fatigue life predictions improved over the elastic superposition method. However, LaRue et al. did not present results of the evolution of the residual stresses during the FCG simulation; neither did they discuss the plastic wake effects.

\subsection{Final remarks}

- The FCG is definitely affected by the presence of pre-existing residual stresses in components and structures, as reflected in the literature. However, it is not an easy task to quantitatively analyze the effect of the pre-existing RSF in the FCG process.

- A reliable prediction of the FCG rates in RSF for damage tolerance assessment remains an open problem. Neither the elastic superposition method nor effective stress intensity factor due to crack closure have proved to be ideal approaches for the prediction of the FCG rates in RSF.

- The initial residual stresses in the un-cracked component are used in the elastic superposition method so that the evolution of the residual stresses during the crack growth is not considered in this method.

- The effective stress intensity factor range method relies on the experimental measurements of the crack opening stresses during the FCG tests which are difficult to accomplish. 
- The experimental methods reported in the literature confirm the redistribution of the residual stresses during the crack growth and the crack closure effect due to the plastic deformation left in the path of the FCG.

\subsection{Objectives}

Based on the previous remarks, the objective of the present manuscript is to develop a numerical model to analyze the FCG process in the components with pre-existing residual stresses. According to the literature review, the model should be capable of:

1. Introducing pre-existing residual stresses into the component, in agreement with experimental measurements.

2. Analyzing the evolution of the pre-existing residual stresses in the component as a function of the FCG.

3. Analyzing the crack closure and plastic wake effects due to the FCG. 


\section{Chapter: Modeling of Residual Stress Fields and Experimental}

\section{Verification}

Previous studies have shown that an accurate characterization of the RSF is crucial for the analysis of the relationship between FCG and residual stresses $[65,66,59,56]$. The main approach used in the literature to model RSF is to simulate the physical process that causes the residual stresses in the specimen. For instance, Kokaly et al. [65] and Jones and Dunn [56] used an elastic-plastic FEA model to simulate the cold-hole expansion

process to determine the RSF. There are other approaches found in the literature to model RSF based on the eigenstrain concept [67], which is a second-order tensor of strain field that represents the plastic deformation due to manufacturing operations. However, the eigenstrain field concept was not used in this manuscript.

This chapter presents a 3-D FEA model used to simulate a four-point bending process and determine the RSF due to elastic-plastic strain incompatibilities on an unnotchedbeam specimen. 7050-T7451 aluminum alloy was considered as the base material for the unnotched-beam specimen. The numerical results were verified against experimental four point bending tests that were designed as part of this research, but were performed by a fellow [68] of our research group at the Technical University of Delft, Netherlands.

\subsection{Material characterization}

A rolled plate of 7050-T7451 aluminum alloy with a thickness of $50 \mathrm{~mm}$ was employed as the base material for the current research. 7050-T7451 is widely employed in the 
aerospace industry to fabricate fuselage frames and bulkheads [69]. For instance, the Boeing 777, the F/A-18 Hornet and the F-22 Raptor aircraft employed the 7050-T7451 aluminum alloy instead of the 7075-T651 version, because its toughness and stress corrosion cracking resistance are superior while conserving an adequate strength [70]. Properties of this material are a function of the thermo-mechanical treatment of the alloy, which is specified by the temper designation (T7451). The T7451 specification indicates that the alloy has been solutionized at high temperature, stress relaxed through a stretching process, quenched and artificially overaged [71, 69]. Table 3-1 and Table 3-2 present the standard chemical composition and the typical mechanical properties for the 7050-T7451 aluminum alloy [72]. However, actual mechanical properties for a specific material batch can be different from the typical ones because of small variations in the chemical composition and thermo-mechanical processing.

Table 3-1 Standard chemical composition of 7050-T7451 aluminum alloy expressed in weight percentage [72].

\begin{tabular}{|l|c|}
\hline Copper, $\mathrm{Cu}$ & $2.0-2.6$ \\
\hline Magnesium, $\mathrm{Mg}$ & $1.9-2.6$ \\
\hline Manganese, $\mathrm{Mn}$ & $0.10 \mathrm{max}$ \\
\hline Silicon, $\mathrm{Si}$ & $0.12 \mathrm{max}$ \\
\hline Iron, $\mathrm{Fe}$ & $0.15 \mathrm{max}$ \\
\hline Chromium, $\mathrm{Cr}$ & 0.04 max \\
\hline Zirconium, $\mathrm{Zr}$ & $0.08-0.15$ \\
\hline Zinc, $\mathrm{Zn}$ & $5.7-6.7$ \\
\hline Titanium, Ti & 0.06 max \\
\hline Other, each & 0.05 \\
\hline Others, total & 0.15 \\
\hline Aluminum, $\mathrm{Al}$ & Balance \\
\hline
\end{tabular}


Table 3-2 Typical mechanical properties of 7050-T7451 aluminum alloy [72].

\begin{tabular}{|l|l|}
\hline Elastic Modulus, $E[\mathrm{GPa}]$ & 70.3 \\
\hline Poisson's ratio & 0.33 \\
\hline Yield strength, $\sigma_{0.2 \%}[\mathrm{MPa}]$ & 455 \\
\hline Ultimate strength, $\sigma_{u}[\mathrm{MPa}]$ & 510 \\
\hline Plain strain fracture toughness, $K_{I c}\left[\mathrm{MPa} \mathrm{m}{ }^{1 / 2}\right]$ & $35.2^{*}$ \\
\hline * LT orientation & \\
\hline
\end{tabular}

Tension and compression tests were conducted according to standard specifications [73, 74] in order to determine the mechanical properties for the base material employed in this research. The specimens were cut from the 7050-T7451 aluminum alloy plate oriented in the longitudinal direction with respect to the rolling direction. The tension test employed a rectangular specimen with a gage section of $50 \mathrm{~mm} \times 12.5 \mathrm{~mm} \times 12.7 \mathrm{~mm}($ length $\times$ width $\times$ thickness), while the compression test employed a cylinder specimen with a length of $60.08 \mathrm{~mm}$ and diameter of $18.04 \mathrm{~mm}$. The thickness of the rectangular specimen for the tension test $(12.7 \mathrm{~mm})$ was also the actual thickness employed for all the specimens analyzed by this research. Figure 3-1 presents the experimentally determined stress-strain curve and mechanical properties for 7050-T7451 aluminum alloy. 


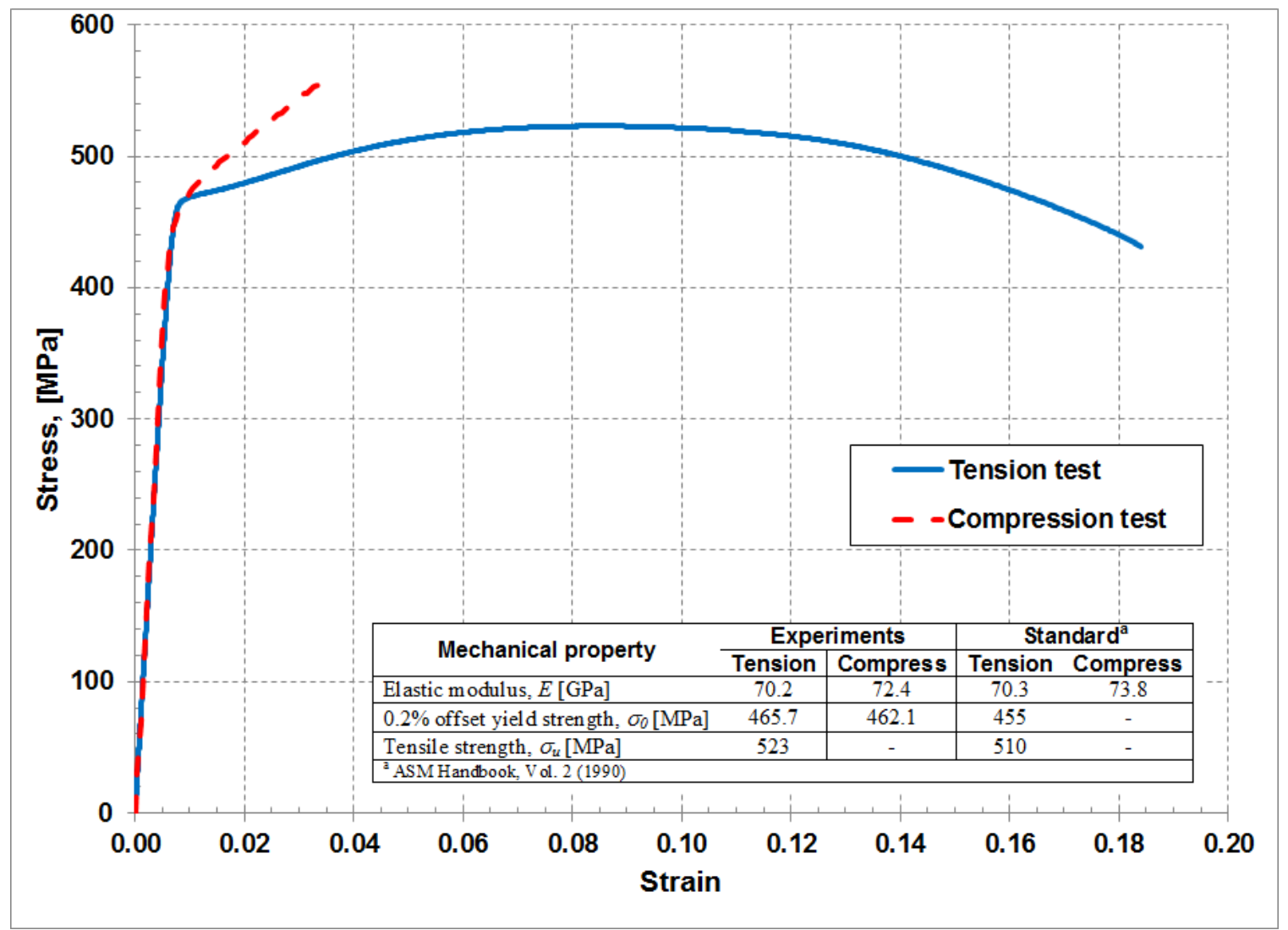

Figure 3-1 Engineering stress-strain curve for 7050-T7451 aluminum alloy.

\subsection{Test setup for the generation of a well-controlled residual stress field in a rectangular specimen}

A well-controlled, reliable and characterized residual stress field is necessary to analyze the FCG in specimens with residual stresses. Residual stresses are an intrinsic characteristic from the thermo-mechanical processing of the 7050-T7451 aluminum alloy plate. In spite of stretching process that is specified in the temper designation for the 7050-T7451 plate, the residual stresses are not completely relaxed and they are present in low magnitudes in the plate. For instance, Figure 3-2 presents the typical condition of pre-existing residual stresses in a plate of 7050-T7451 aluminum alloy according to [75]. 
Residual stresses are shown as a function of the z-axis that is oriented along the thickness of the plate. In this manuscript, the pre-existing residual stresses in the as-received condition of the 7050-T7451 aluminum alloy plate were regarded as negligible, because a simple mechanical process was employed to generate a new pre-existing residual stress field. The mechanical process consisted of a non-uniform plastic deformation, where preexisting residual stresses in the as-received condition of the 7050-T7451 aluminum alloy plate were overwritten by a new field.

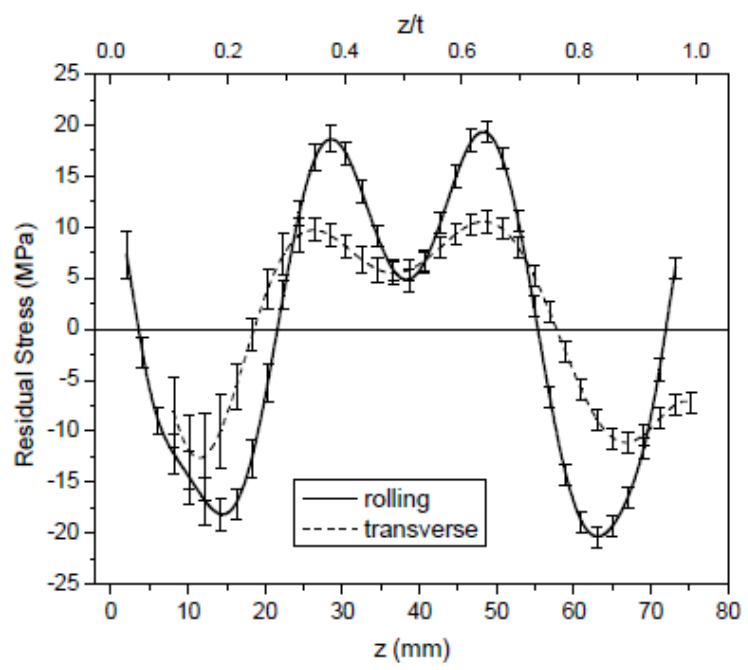

Figure 3-2 Pre-existing residual stress fields in a 7050-T7451 aluminum plate. (Reprinted from [75] )

The pre-existing residual stress field analyzed by this manuscript was generated with a four-point bending test, which was applied to an unnotched beam specimen with a rectangular geometry. Figure 3-3 presents the unnotched beam specimen and the fourpoint bending test setup. For the fixed specimen geometry and material, the residual stress field was just dependent on the applied bending load provided that plastic 
deformation was induced over the top and bottom regions of the unnotched beam specimen (Figure 3-3).

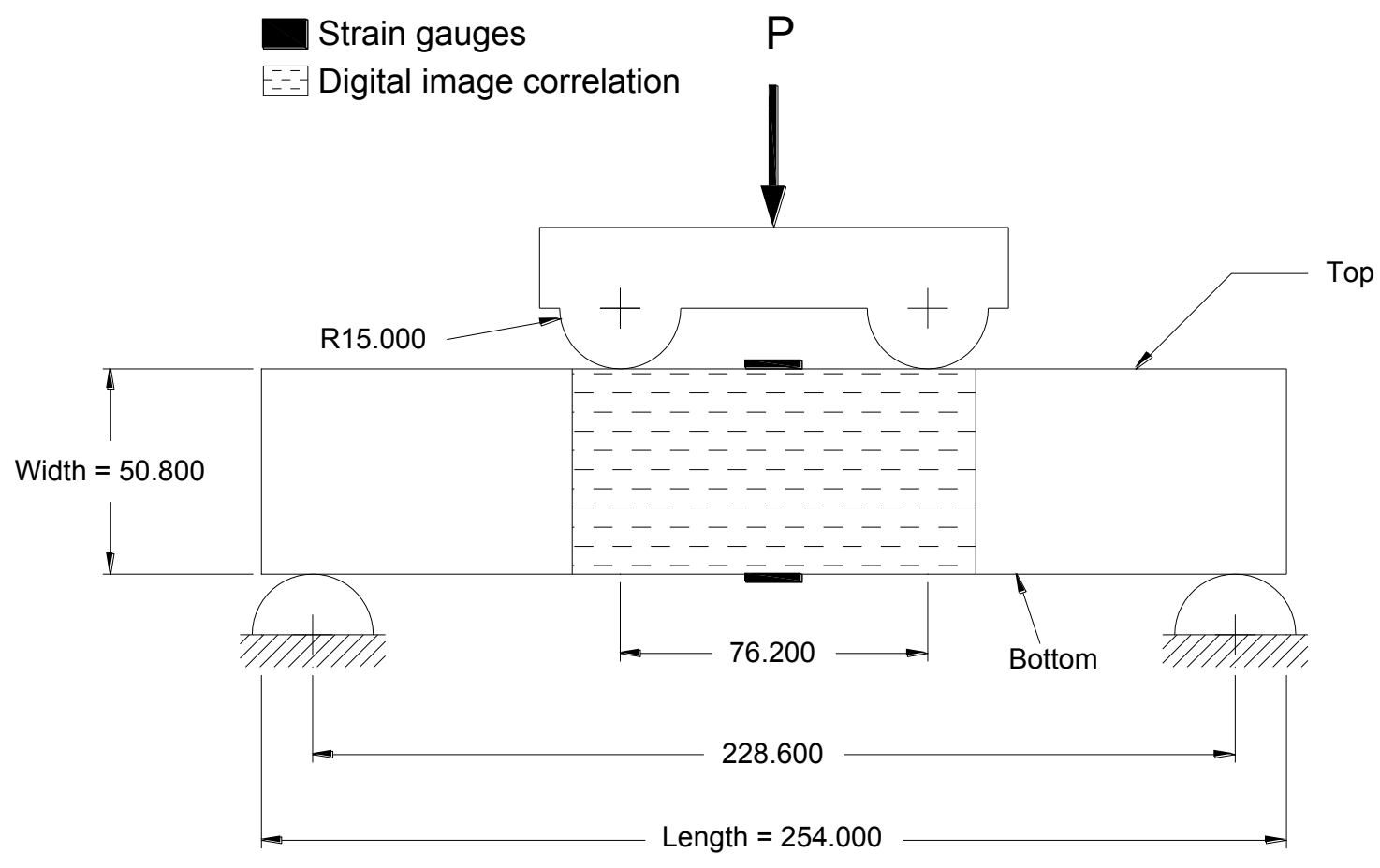

Specimen's thickness: $12.7 \mathrm{~mm}$

Dimensions: $\mathrm{mm}$

Figure 3-3 Four-point bending test setup and unnotched-beam specimen dimensions.

The four-point-bending test was designed to introduce a strain of $10,000 \mu \varepsilon$ at the maximum applied load in the top material region of the unnotched beam specimen, i.e. for the specimen's material and geometry equivalent to a load of $\sim 90 \mathrm{kN}$ according to standard bending theory [76]. The plastic deformations induced over the top and bottom regions of the unnotched beam specimen prevent the complete recovery of the elastic 
deformations in the center region of the specimen. As a result, RSF arise from the incompatibility between elastic and plastic strains within the unnotched beam.

The mechanical process described in Figure 3-3 was modeled in the commercially available finite element software ABAQUS ${ }^{\mathrm{TM}}$ in order to determine the RSF. The finite element model created for the simulation is explained in the next section of this chapter. In addition, an experimental test plan was developed to verify the finite element results of the simulation. However, the actual experimental tests were conducted at Delft University of Technology (TU Delft) by Lotz as part of his M. Sc. Thesis. The next paragraphs present a brief description of the experimental study for completeness. Experimental four-point bending tests were conducted on a universal testing machine that was manually operated at a displacement rate of $\sim 9 \mu \mathrm{m}$ per second ( $0.54 \mathrm{~mm}$ per minute). The applied load in the four-point bending test was monitored in real time and the strain was measured at each applied load increment $\Delta P=2 \mathrm{kN}$. The four-point bending test was conducted until the lower strain gauge on the specimen reached (Figure 3-3) approximately $10,000 \mu \varepsilon$. Digital image correlation and slitting experimental methods were employed to determine residual stress and strain fields introduced during the fourpoint bending tests, in addition to serving as a means of verifying the finite element results. Table 3-3 presents a description of the specimens subjected to the four-point bending test and the experimental method employed to measure the residual strain and stress fields. The Digital Image Correlation (DIC) technique is an optical experimental method used to measure surface displacements on a component subjected to external loads $[77,78,79]$. DIC employs high-resolution digital images and an image correlation 
algorithm in order to compute the full-field displacements between a reference image of the specimen and a loaded condition.

Table 3-3 Specimens, four-point bending test conditions and experimental methods.

\begin{tabular}{|c|c|c|c|c|}
\hline \multirow{2}{*}{ Specimen Id. } & \multirow{2}{*}{ Max. Load [kN] } & \multicolumn{2}{|c|}{ Bottom gauge, [ $\boldsymbol{\mu} \boldsymbol{\varepsilon}]$} & \multirow{2}{*}{ Experimental method } \\
\cline { 3 - 4 } & & Max. Load & Unload & \\
\hline B2 & 90.82 & 10125 & 1493 & DIC and Slitting \\
\hline B3 & 89.62 & 10024 & 1429 & DIC \\
\hline B4 & 88.29 & 10117 & 1465 & DIC and Slitting \\
\hline
\end{tabular}

During the experimental four-point bending tests, two cameras of 4 Megapixels were used to capture several images and employ the DIC technique. A speckle pattern was applied to one face of the specimens prior to the four point bending test to ensure that each subset of pixels in the image were different between them. Further, a white light source was focused on the spackle pattern to ensure an adequate contrast between each subset of pixels in the images. The region over the specimen where the speckle pattern was applied is also presented in Figure 3-3. The cameras were set up to capture one speckle by approximately 3 pixels in the digital image. The analysis was done with a subset size of 21 pixels and a step size of 10 pixels in the VIC3D software (Correlated Solutions Inc., Columbia, SC, USA). In addition, specimens B2 and B4 were subjected to a slitting method to independently verify the RSF for our four-point bending process. The slitting method is regarded as a well-established method to determine through-the-thickness residual stresses normal to a cut plane [80]. The slitting method is a compliance technique where the specimen is cut by increments while a surface strain gauge measures the resulting strains as a function of the cut increment [81]. The specimens B2 and B4 were placed on a cantilever 
configuration and were cut with Electro-Discharge Machining (EDM) at the mid-section of the specimen's length (Figure 3-3). Strains as a function of the cut depth were measured by the bottom gauge of the unnotched beam specimen. The RSF were assumed to be a linear combination of a known stress distribution represented by Legendre polynomials. The published available data from [82] for Legendre orders 2-12 was used as compliance functions to determine the RSF for specimens B2 and B4.

\subsection{Elastic-plastic finite element model of the four point bending test}

The finite element program ABAQUS ${ }^{\mathrm{TM}}$ Vr. 6.11-2 was employed to simulate the fourpoint bending test and thus compute the pre-existing RSF in the unnotched-beam model. Figure 3-4 presents the finite element model employed to determine the RSF. It consists of the unnotched beam specimen and the upper and lower rollers. Boundary symmetry conditions along the mid-section of the specimen's length were employed so only half of the four-point bending test was modeled to reduce the modeling complexity and the required computing resources. Further, the rollers were modeled as rigid bodies, because it was assumed that they do not deform during the four-point bending test. 


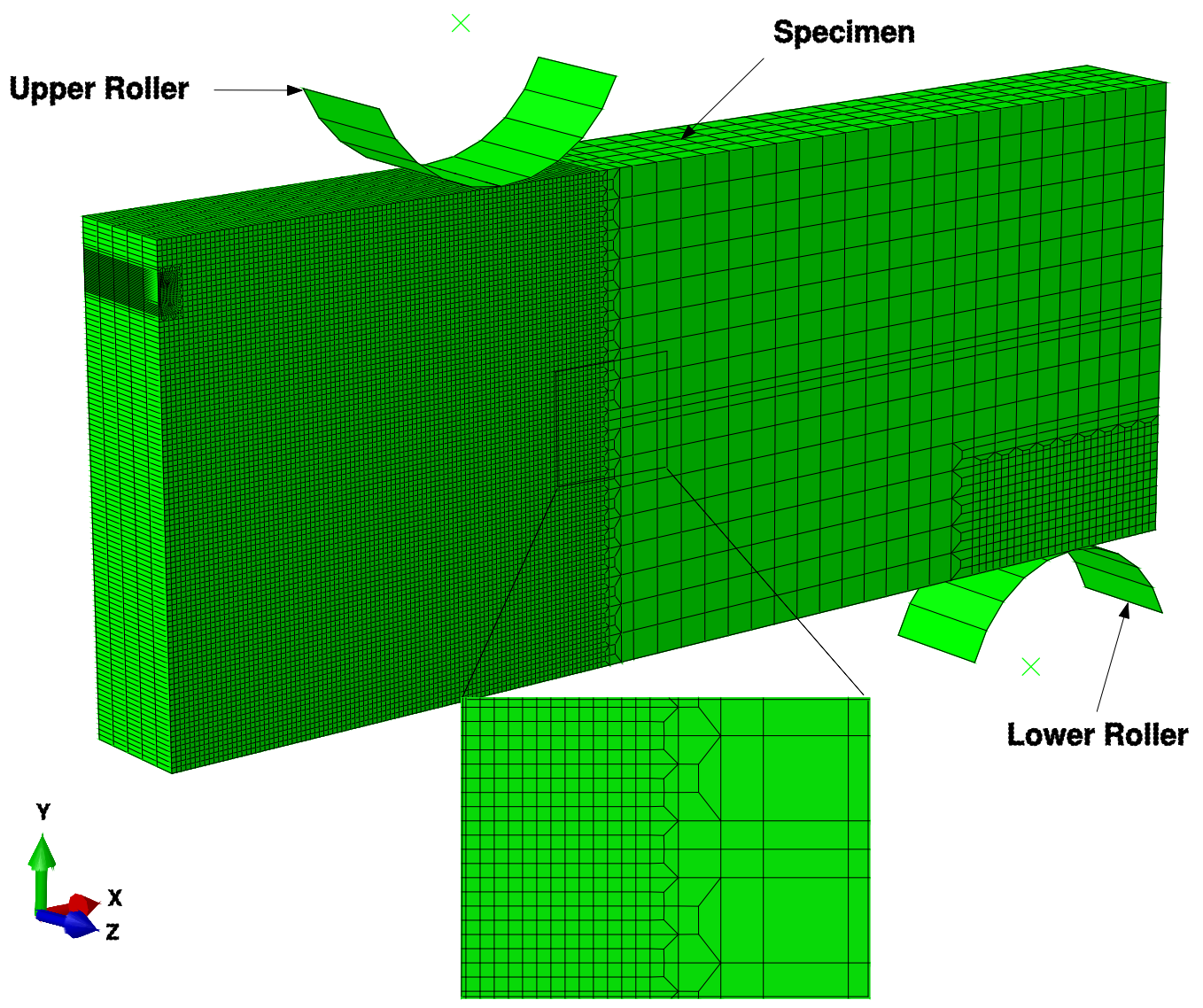

Figure 3-4 Finite element model to compute the residual stress fields.

A structured mesh of 8-node linear brick elements was employed for the unnotched specimen and a reduced integration scheme was employed to compute the stress and strain fields. The reduced integration scheme in ABAQUS ${ }^{\text {TM }}$ employs just one integration point for the 8-node linear brick elements (C3D8R). The mesh of the unnotched specimen was refined for a region close to the symmetry plane. Figure 3-4 also presents the mesh refinement. The element size in the refined mesh was set to $0.508 \mathrm{~mm}$ with an aspect ratio of 1 for the plane $x-y ; 5$ elements were employed along the $z$-direction (thickness); and the total number of elements in the mesh of the unnotched specimen was 56,010 (C3D8R). A surface-to-surface contact interaction was defined between the rollers and 
the specimen during the four-point bending test simulation. The tangential behavior of the contact interaction was set as frictionless so nodal displacements along the $x$-direction were not constrained, but the normal behavior of the contact interaction was set to transmit a contact-pressure so nodal displacements along the $y$-direction were penalized. The rigid surface on the roller was defined as a master surface and its counterpart surface on the specimen was defined as the slave surface. The four-point-bending test simulation was accomplished in three time steps; the initial time step was set to apply a small displacement on the upper roller along the negative $y$-axis (Figure 3-4) to initiate the contact between the rollers and the specimen; the maximum load was incrementally applied to the upper roller in the second time step; and a final time step was set to release the load, after which the misfit plastic strains of the inhomogeneous bending deformation resulted in a well-known RS field in the beam specimen.

The von Mises yield function and a combined hardening model were employed for the elastic-plastic material behavior during the four-point bending test simulation. The combined hardening rule is a combination of the isotropic and the kinematic hardening rules, which are presented in Figure 3-5. In the case of the isotropic hardening rule, the subsequent yield surface expands with respect to the previous one while conserving the same center as a function of the plastic deformation. Besides, the yield point under a reversal yielding is the same as the initial one (Figure 3-5; B = E). In the case of the kinematic hardening rule, the subsequent yield surface is only a translation with respect to the previous one while conserving the same size as a function of the plastic deformation. Further, the yield point under a reversal yielding is considerable lower than the initial yield point (Figure 3-5; C $<\mathrm{B}$ ). In the case of the combined hardening rule, the 
subsequent yield surface is an expansion and translation with respect to the previous one as a function of the plastic deformation so the subsequent yield surface has a different center and size. In addition, the yield point under a reversal yielding is lower than the corresponding one from the isotropic hardening model, but it is larger than the corresponding one from the kinematic hardening model (Figure 3-5; $\mathrm{C}<\mathrm{D}<\mathrm{E}$ ).

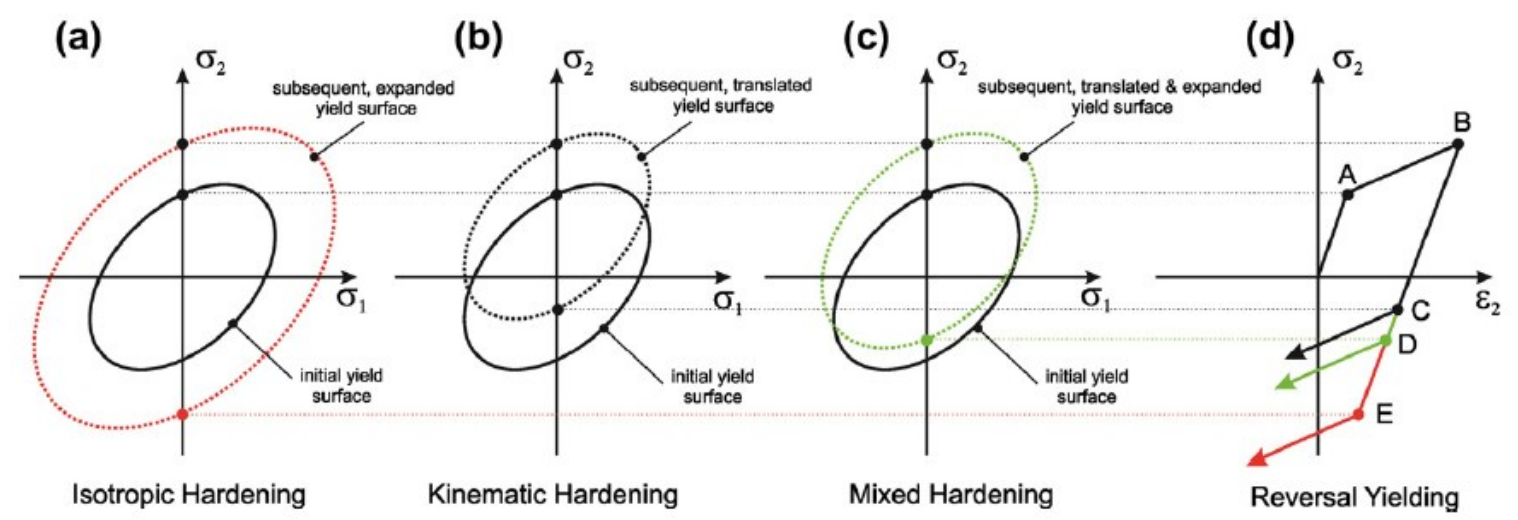

Figure 3-5 a) Isotropic hardening rule; b) kinematic hardening rule; c) combined hardening rule; and d) yield points under stress reversal for each hardening rule.

The material properties determined by the uniaxial tension test (Figure 3-1) were employed to define the elastic-plastic material behavior. The elastic modulus $E$ was 70 $\mathrm{GPa}$ and the Poisson's ratio was 0.33 . Figure 3-6 presents the coordinates of the true stress-strain data points employed to determine the material input parameters for the combined hardening model in ABAQUS ${ }^{\mathrm{TM}}$, along with the true stress-strain curve computed from the engineering stress-strain curve of 7050-T7451 aluminum alloy (Figure 3-1). 


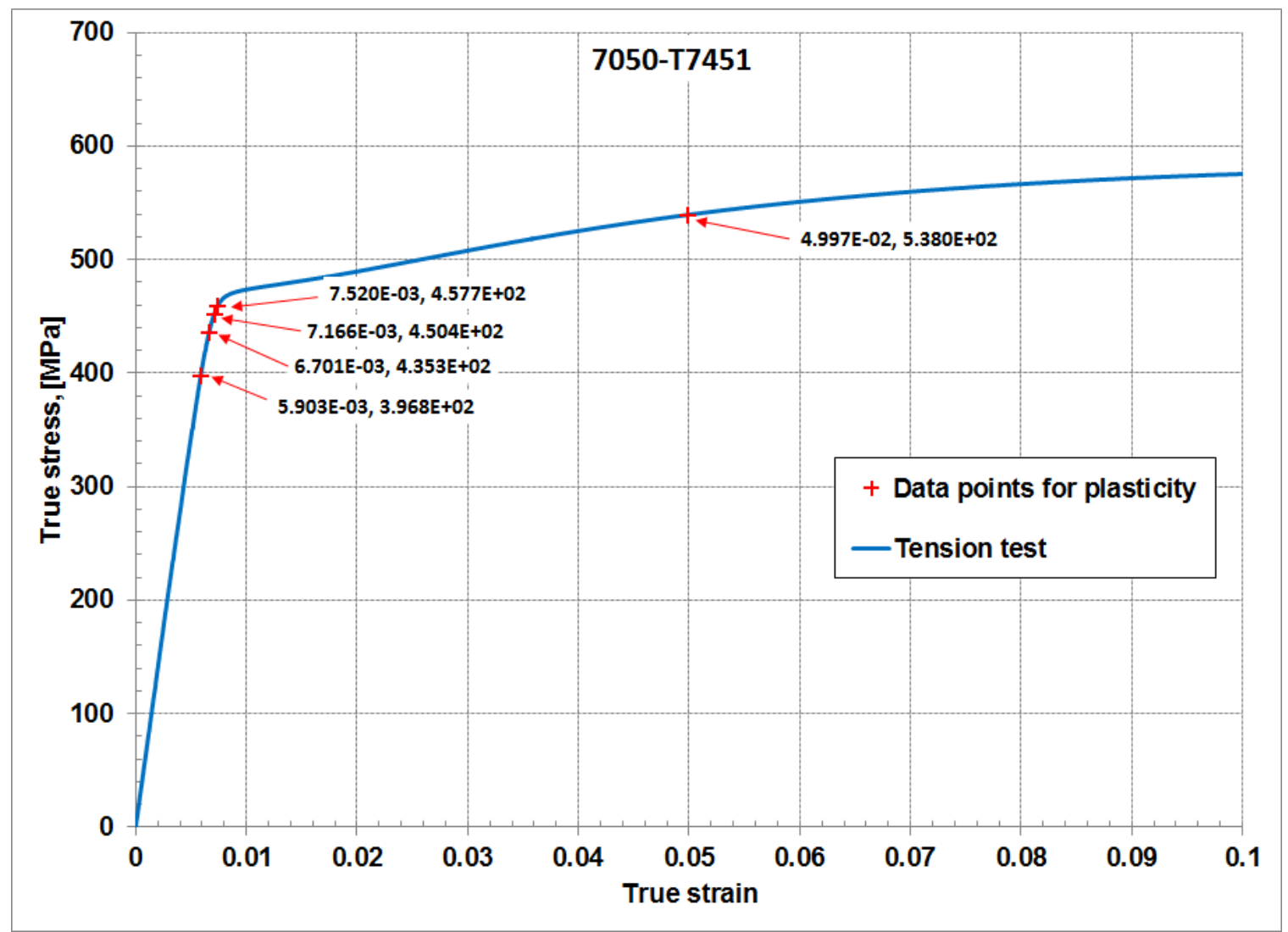

Figure 3-6 True stress-strain curve and material data points for the combined hardening model used in the FEA models.

The material data points of Figure 3-6 were defined with the offset method [83] at 0.025, $0.05,0.075,0.1$, and $4 \%$ proof stress. The combined hardening model in ABAQUS ${ }^{\mathrm{TM}}$ was specified by a set of true stress and plastic strain components $\left(\sigma_{n}, \varepsilon_{n}^{p}\right)$. Table 3-4 presents the material input parameters employed in ABAQUS ${ }^{\mathrm{TM}}$ for the elastic-plastic behavior of 7050-T7451 aluminum alloy. 
Table 3-4 The material model parameters for elastic-plastic behavior of 7050-T7451 aluminum alloy in ABAQUSTM.

\begin{tabular}{|l|l|}
\hline \multicolumn{1}{|c|}{ Parameter } & \multicolumn{1}{c|}{ Value } \\
\hline$E$ & $70.217 \mathrm{GPa}$ \\
\hline$v$ & 0.33 \\
\hline$\left(\tilde{\sigma}_{0}, \tilde{\varepsilon}_{0}^{p}\right)$ & $396.8 \mathrm{MPa}, 0.0^{\mathrm{a}}$ \\
\hline$\left(\tilde{\sigma}_{1}, \tilde{\varepsilon}_{1}^{p}\right)$ & $435.3 \mathrm{MPa}, 0.000502$ \\
\hline$\left(\tilde{\sigma}_{2}, \tilde{\varepsilon}_{2}^{p}\right)$ & $450.4 \mathrm{MPa}, 0.000750$ \\
\hline$\left(\tilde{\sigma}_{3}, \tilde{\varepsilon}_{3}^{p}\right)$ & $457.7 \mathrm{MPa}, 0.001001$ \\
\hline$\left(\tilde{\sigma}_{4}, \tilde{\varepsilon}_{4}^{p}\right)$ & $538.0 \mathrm{MPa}, 0.042318$ \\
\hline${ }^{\mathrm{a}}$ Zero plastic strain component at initial yield point. \\
\hline
\end{tabular}

The elastic-plastic finite element model (Figure 3-4) and the material model parameters (Table 3-4) were also employed in the next chapter for the analysis of the FCG in RSF.

\subsection{Results of strain/stress fields calculation}

The strain field produced by the maximum applied load during the four-point bending test is presented in Figure 3-7 according to the FEA simulation (unnotched-beam model) and the experimental measurements with DIC technique (specimen B3). Both, the FEA model and the test specimen were subjected to the same conditions (Figure 3-3) during the four-point bending test. Figure 3-8 presents the results of the residual strain field left in the unnotched-beam model (FEA simulation) and the specimen B3 (DIC measurements) after the four-point bending test. The symmetry plane on the unnotchedbeam model is also shown in Figure 3-8a as a reference for the next figure. A plot of the 1-D residual strain distribution as a function of the $y$-coordinate along the symmetry plane of the unnotched beam model is shown in Figure 3-9. In addition, the 1-D residual 
strain distribution determined by the DIC technique and point strain measurements determined by strain gauges are presented in Figure 3-9 for test specimens B2, B3 and B4. The experimental measurements were performed along the middle of the specimen's length (Figure 3-3), which coincided with the symmetry line on the unnotched-beam model.
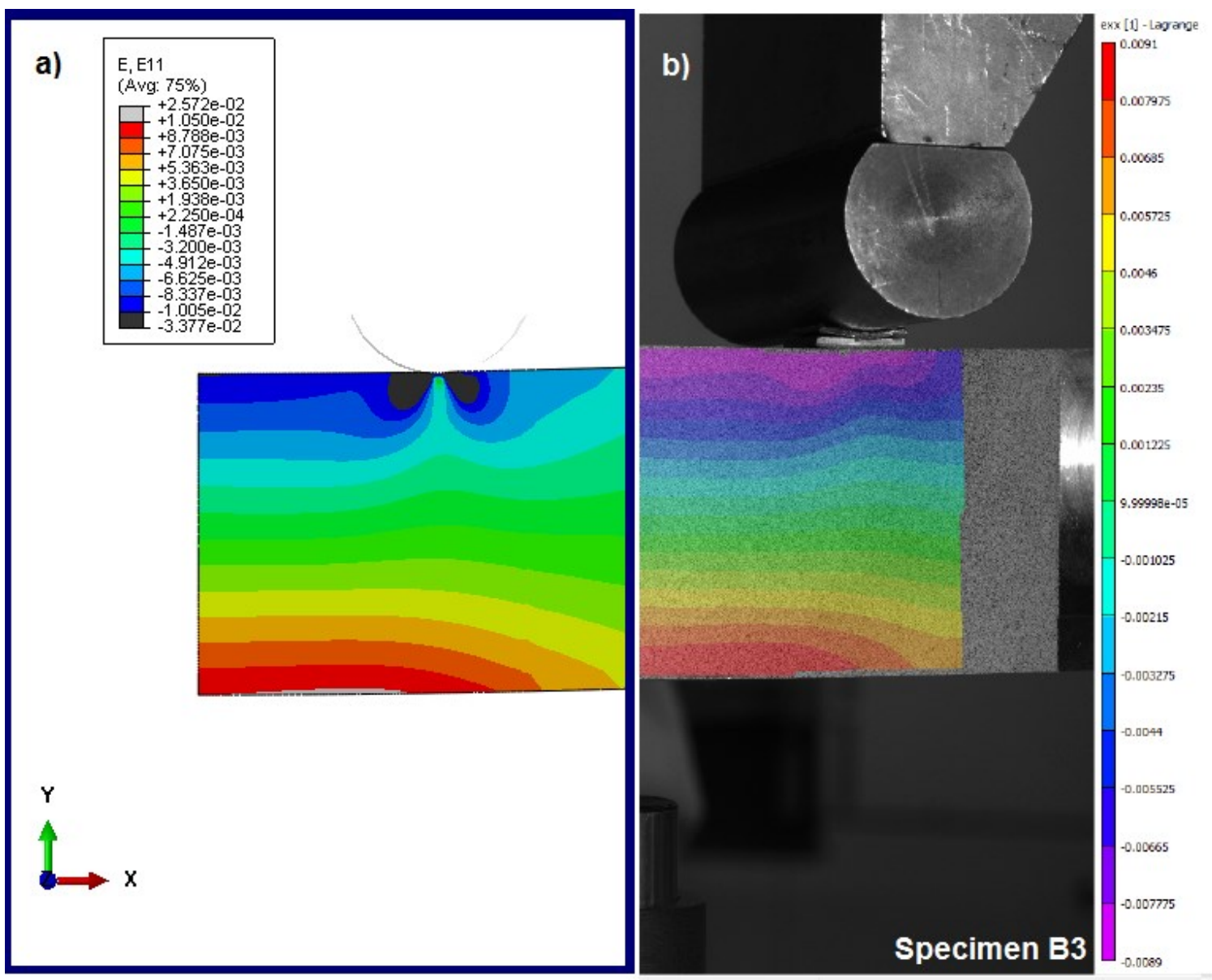

Figure 3-7 Strain field produced by the maximum applied load during the four-point bending test according to the FEA simulation and the experimental measurement: a) unnotched-beam model and b) DIC technique on test specimen B3. 

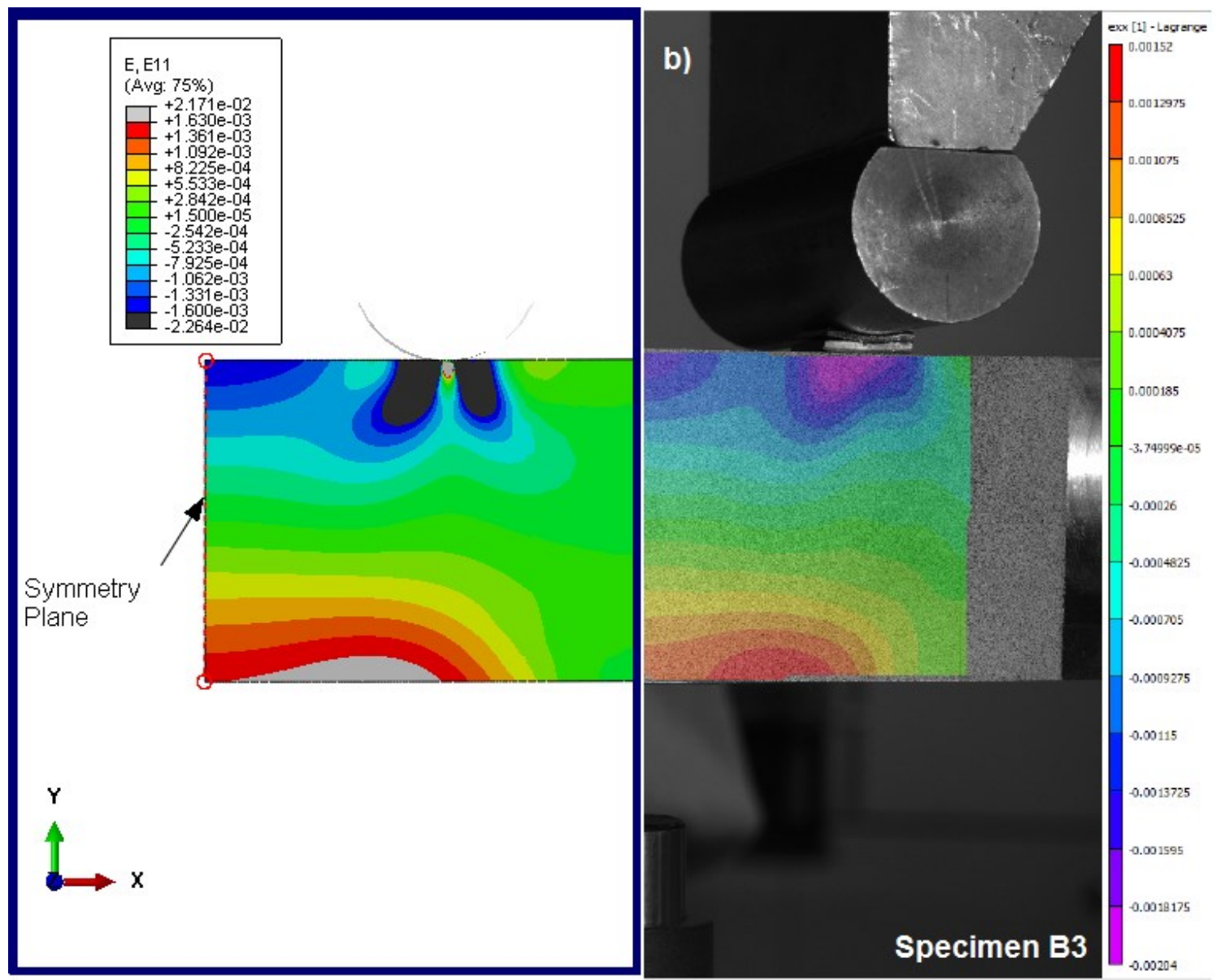

Figure 3-8 Residual strain field after the four-point bending test: a) unnotched-beam model and b) DIC technique on test specimen B3. 


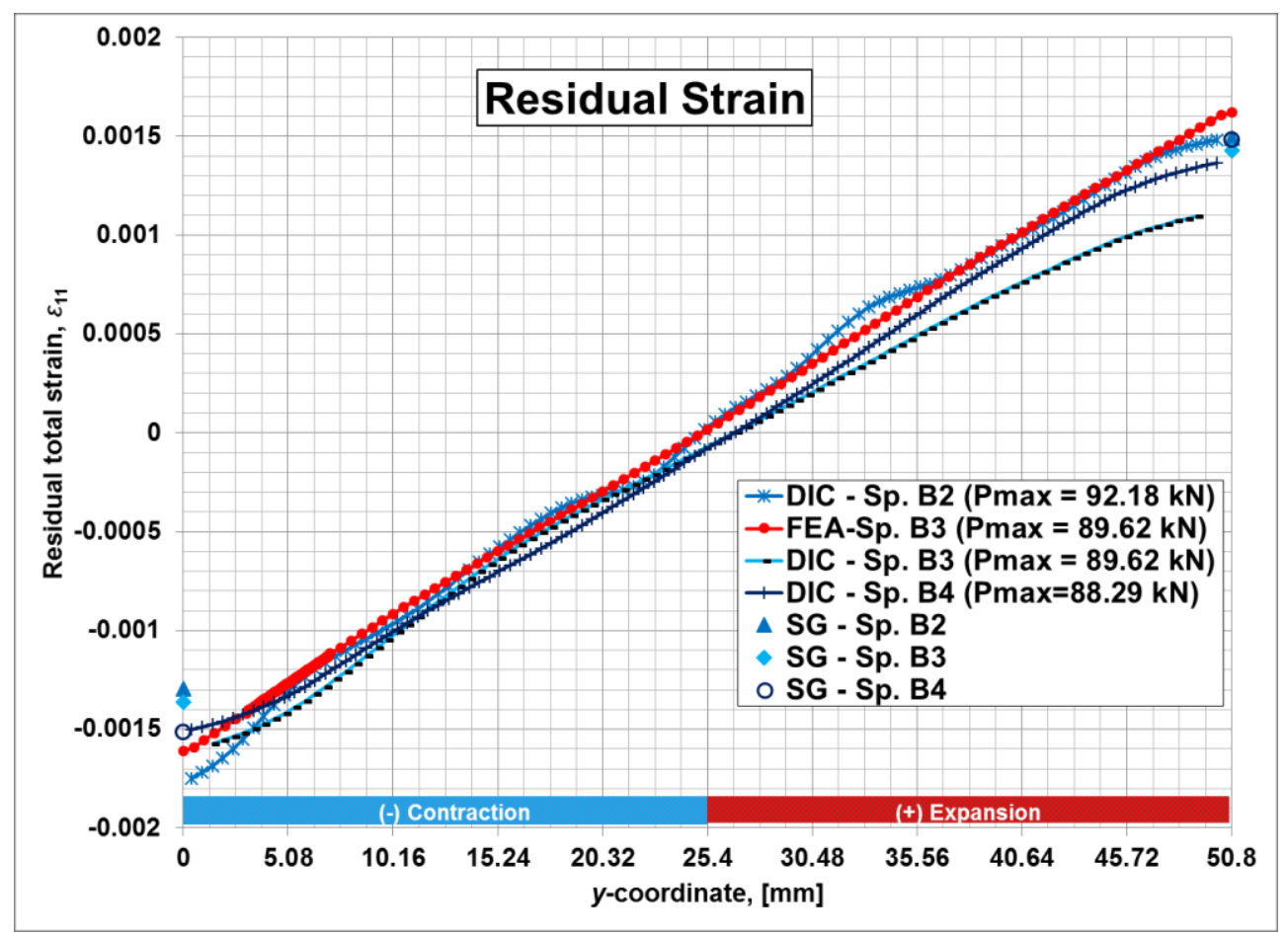

Figure 3-9 1-D distribution of residual strain in the unnotched-beam model (FEA analysis) and test specimens B2, B3 and B4 (DIC technique). Strain point measurements with Strain Gauges (SG) on test specimens B2,B3 and B4.

The elastic and plastic strain components of the residual strain field on the unnotchedbeam model after the four-point bending test simulation are shown in Figure 3-10 and Figure 3-11. Figure 3-12a presents a contour plot of residual stresses (MPa) on the unnotched-beam model (Figure 3.4) from the final step of the four point bending test simulation, and Figure 3-12b presents the residual stresses as a function of the $y$ coordinate along the symmetry plane on the unnotched-beam model. In addition, the results from the slitting method of the residual stresses produced by the four-point bending process on the mid-section of test specimens $\mathrm{B} 2$ and $\mathrm{B} 4$ are also presented in Figure 3-12b. 


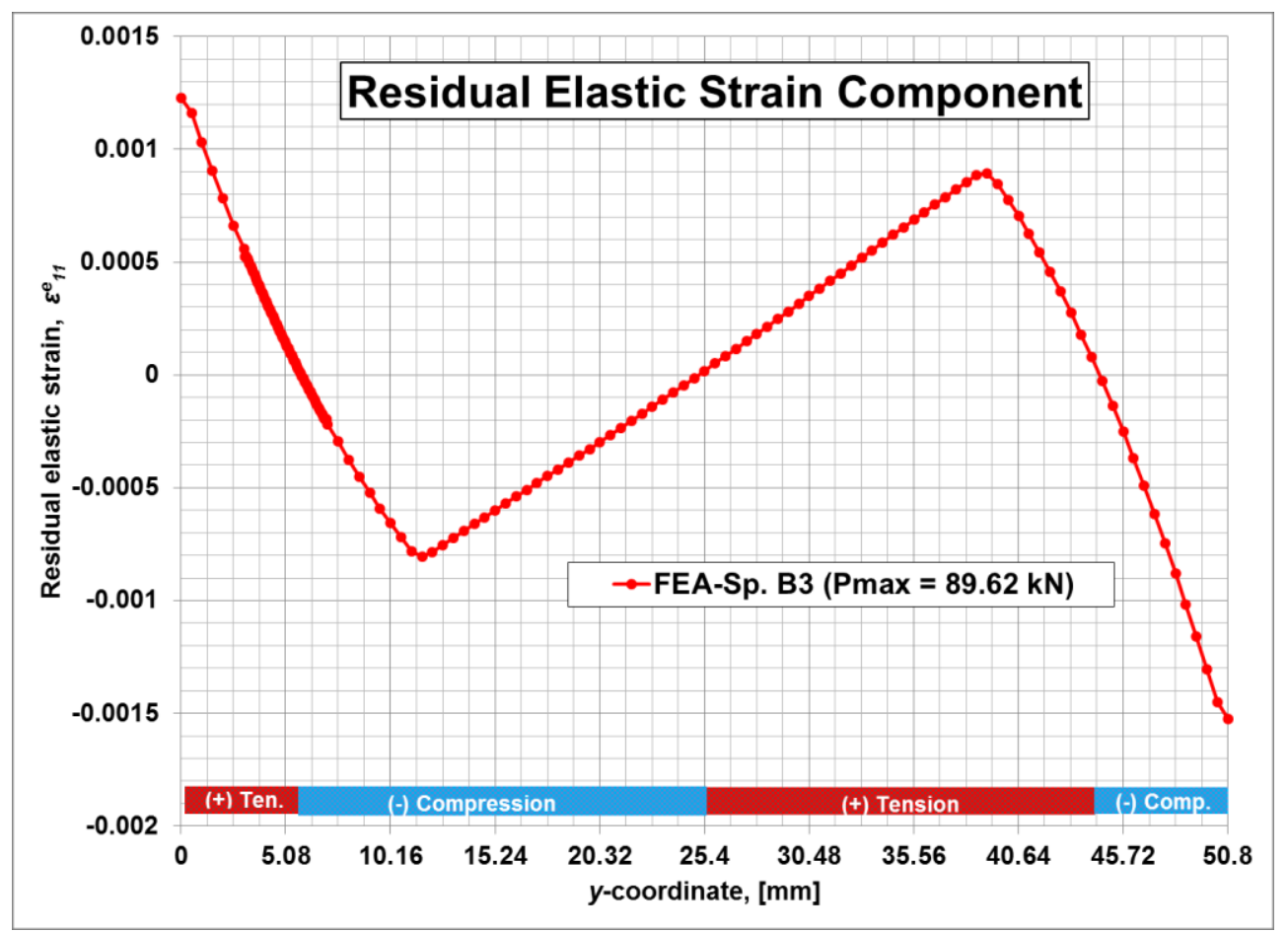

Figure 3-10 Residual elastic strain component according to the finite element results.

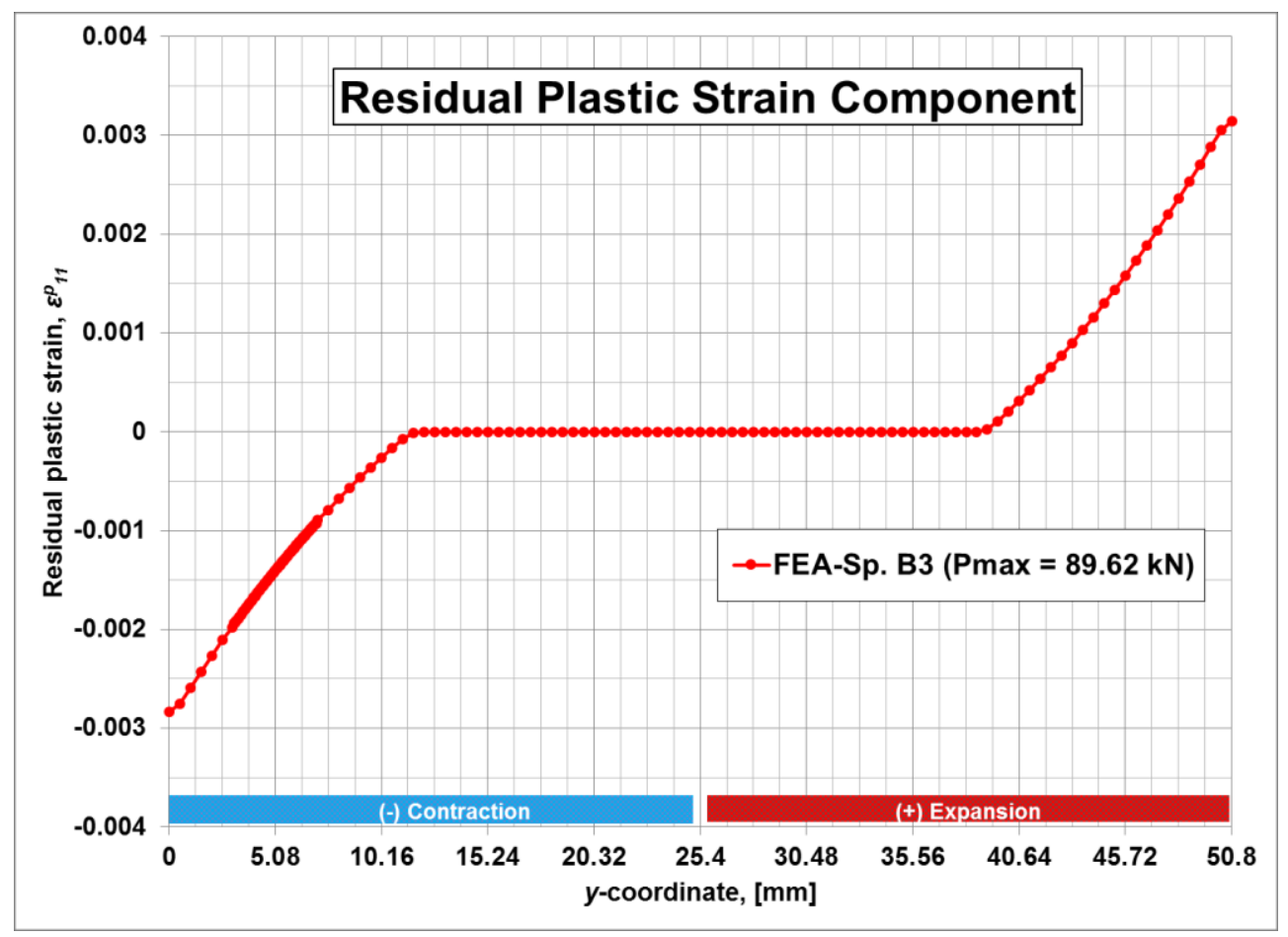

Figure 3-11 Residual plastic strain component according to the finite element results. 

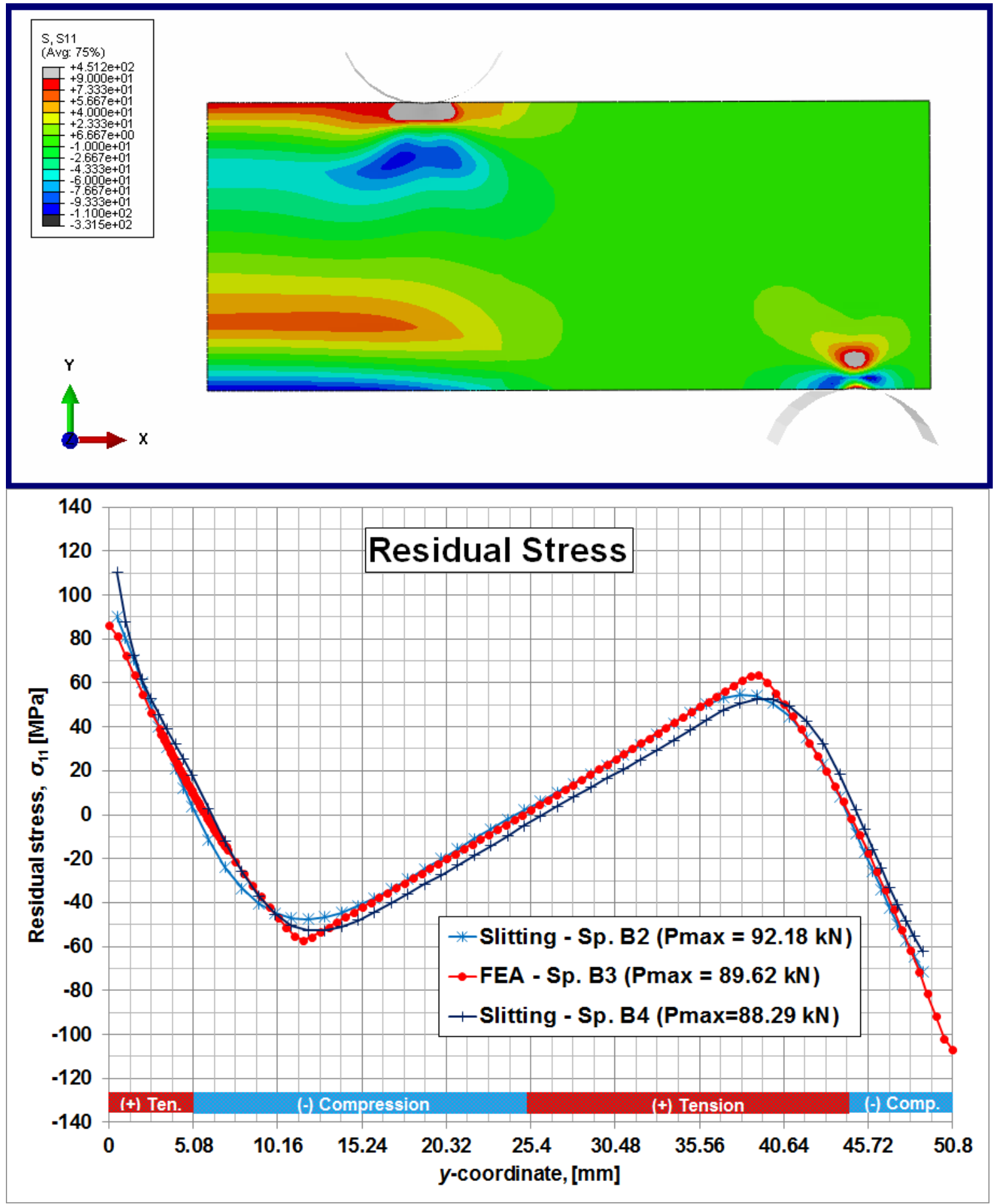

Figure 3-12 a) Contour plot of residual stresses (MPa) produced by the four-pointbending test simulation, and $b$ ) the residual stress field distribution (1-D) as a function of the $y$-coordinate along the plane of symmetry on the unnotched-beam model. 


\subsection{Discussion of residual strain and stress fields due to four point bending test simulation.}

Maximum values of strain and stress produced by the four-point bending process were developed along the normal components in the $x$-direction of an axis of reference as the one shown in Figure 3-7. Thus, the normal components in the $x$-direction were regarded as the residual strain and stress fields analyzed by this study. Moreover, this component corresponded to the direction of the applied load during the FCG analysis conducted in the next section of this manuscript. The remaining components of strain and stress were not analyzed in this study. Material zones adjacent to the lower and upper rollers presented the largest value of strain and stress produced by the four-point bending test, as observed in the contour plot of Figure 3-7a, Figure 3-8a and Figure 3-12a. However, these zones did not correspond to the material region where the crack was introduced for the FCG test simulation.

In the case of the strain produced by the four-point bending process, Table 3-5 summarizes the results of the unnotched-beam model and the test specimen B3. The results are reported according to the legend in the contour plots of Figure 3-7 and Figure 3-8. The FEA results indicated a value of the strain (Figure 3-7a), produced by the maximum applied load during the four-point bending simulation, of $-1.005 \mathrm{e}-2(-10,050$ $\mu \varepsilon)$ and $1.050 \mathrm{e}-2(10,500 \mu \varepsilon)$ for the top and bottom regions on the unnotched-beam model. The DIC results indicated a value of the strain (Figure $3-7 b)$ of $-0.0089(-8,900$ $\mu \varepsilon)$ and $0.0091(9,100 \mu \varepsilon)$ for the top and bottom regions on the test specimen B3. Regarding the residual strain left in the unnotched-beam specimen after the four-point 
bending test, the FEA results indicated a variation from its top region of -1.600e-3 (1,600 $\mu \varepsilon)$ to its bottom region of $1.630 \mathrm{e}-3(1,630 \mu \varepsilon)$. Meanwhile, the DIC results indicated a variation from the top region of the test specimen B3 of approximately $-0.001595(-1,595$ $\mu \varepsilon)$ to its bottom region of approximately $0.001075(1,075 \mu \varepsilon)$.

Table 3-5 Numerical and experimental results of the strain produced by the four-point bending.

\begin{tabular}{|c|c|c|c|c|c|}
\hline \multirow{2}{*}{ Field } & \multirow{2}{*}{$\begin{array}{l}\text { Specimen's } \\
\text { region }\end{array}$} & \multicolumn{2}{|c|}{ Result } & \multicolumn{2}{|c|}{ Reference Color } \\
\hline & & $F E A$ & $D I C$ & $F E A$ & $D I C$ \\
\hline \multirow{2}{*}{$\begin{array}{l}\text { Strain produced by } \\
\text { the maximum } \\
\text { applied load } \\
\text { (Figure 3-7) }\end{array}$} & Top & $\begin{array}{l}-1.005 \mathrm{e}-2 \\
(-10,050 \mu \varepsilon)\end{array}$ & $\begin{array}{l}-0.0089 \\
(-8,900 \mu \varepsilon)\end{array}$ & Blue & Magenta \\
\hline & Bottom & $\begin{array}{l}1.050 \mathrm{e}-2 \\
(10,500 \mu \varepsilon)\end{array}$ & $\begin{array}{l}0.0091 \\
(9,100 \mu \varepsilon)\end{array}$ & Red & Red \\
\hline \multirow{2}{*}{$\begin{array}{l}\text { Residual strain } \\
\text { (Figure 3-8) }\end{array}$} & Top & $\begin{array}{l}-1.600 \mathrm{e}-3 \\
(-1,600 \mu \varepsilon)\end{array}$ & $\begin{array}{l}-0.001595 \\
(-1,595 \mu \varepsilon)\end{array}$ & Blue & Violet \\
\hline & Bottom & $\begin{array}{l}1.630 \mathrm{e}-3 \\
(1,630 \mu \varepsilon)\end{array}$ & $\begin{array}{l}0.001075 \\
(1,075 \mu \varepsilon)\end{array}$ & Red & Orange \\
\hline
\end{tabular}

The distribution of residual strain as a function of the $y$-coordinate along the symmetry line on the unnotched-beam model was linear, as shown in Figure 3-9. Table 3-6 summarizes the maximum and minimum values of the 1-D residual strain distribution exhibited in Figure 3-9. The negative values for the minimum residual strain distribution indicated a contraction of the material in this region, while the positive values for the maximum residual strain distribution indicated an expansion of the material in this region. The FEA results from the unnotched-beam model indicated a value of the minimum and maximum residual strain distribution of $-0.001608(-1,608 \mu \varepsilon)$ and $0.001622(1,622 \mu \varepsilon)$. In the case of the test specimen B2, the result of the minimum and maximum residual strain distribution were $-0.001749(-1,749 \mu \varepsilon)$ and $0.001481(1,481$ 
$\mu \varepsilon)$ according to the DIC measurements. For the test specimen B3, the minimum and maximum residual strain distribution were $-0.001572(-1,572 \mu \varepsilon)$ and $0.001095(1,095$ $\mu \varepsilon)$. Finally, the result of the minimum and maximum residual strain distribution for test specimen B4 were $-0.0015(-1,500 \mu \varepsilon)$ and $0.001364(1,364 \mu \varepsilon)$. The difference between the unnotched-beam model and the average of the three test specimens (B2, B3 and B4) for the minimum residual strain along the symmetry line was just $1 \mu \varepsilon$, and for the maximum residual strain was $309 \mu \varepsilon$.

Table 3-6 Maximum and minimum values of the 1-D residual strain distribution along the mid-section of the test specimen (symmetry line on the unnotched-beam model).

\begin{tabular}{|c|c|c|c|c|}
\hline \multirow{2}{*}{ Specimen } & \multicolumn{2}{|c|}{ Reference (Figure 3-9) } & \multicolumn{2}{|c|}{ Residual strain } \\
\hline & Line color & Markers & Minimum & Maximum \\
\hline $\begin{array}{l}\text { Unnotched-beam } \\
\text { Model }\end{array}$ & Red & Closed circle & $\begin{array}{l}-0.001608 \\
(-1,608 \mu \varepsilon)\end{array}$ & $\begin{array}{l}0.001622 \\
(1,622 \mu \varepsilon)\end{array}$ \\
\hline B2 & Blue & Asterisks & $\begin{array}{l}-0.001749 \\
(-1,749 \mu \varepsilon)\end{array}$ & $\begin{array}{l}0.001481 \\
(1,481 \mu \varepsilon)\end{array}$ \\
\hline B3 & Cyan & Black hyphen & $\begin{array}{l}-0.001572 \\
(-1,572 \mu \varepsilon)\end{array}$ & $\begin{array}{l}0.001095 \\
(1,095 \mu \varepsilon)\end{array}$ \\
\hline B4 & Dark-blue & Plus & $\begin{array}{l}-0.0015 \\
(-1,500 \mu \varepsilon)\end{array}$ & $\begin{array}{l}0.001364 \\
(1,364 \mu \varepsilon)\end{array}$ \\
\hline
\end{tabular}

The residual strain field (Figure 3-8) can be decomposed into the residual elastic and plastic strain components. The residual elastic strain component presented a zigzag distribution as observed in Figure 3-10, which is analogous to the distribution of residual stresses (Figure 3-12b). The elastic component of the residual strain was characterized by three neutral points of zero strain and two alternating regions of positive strains balanced by negative strains. The computed residual elastic strain by the four point bending test simulation at the peak points were $\sim+0.0012,-0.0008,+0.0009$ and -0.0015 . The zigzag 
distribution of the residual elastic strain is typical of experimental measurements of the lattice strain determined by diffraction techniques $[84,85]$, which are used to measure RSF.

The residual plastic strains were induced at the outer surface of the unnotched-beam model by the maximum applied load and expanded inwards till a depth from the surface of approximately $11.5 \mathrm{~mm}$ as observed in Figure 3-11 c. This depth corresponded to the position of the internal residual stress peaks (-60 and $+65 \mathrm{MPa}$ ) observed in Figure 3-12b. During the elastic unloading, the plastic strains remained constant and prevented the complete recovery of the elastic strains at the center region of the specimen's width. The RSF arose as a consequence of the strain incompatibility between elastic and plastic regions.

The contour plot of Figure 3-12a for the unnotched-beam model showed the overall distribution of residual stresses. An alternating distribution of the RSF in the central region of the unnotched-beam model, with the largest values for material zones adjacent to the rollers was observed. However, the region of interest for the FCG analysis of the next chapter was along the symmetry plane on the unnotched-beam model. This plane was specified as the FCG plane, where a crack was introduced to modify the unnotchedbeam model into a SENT model. The distribution of the residual stresses along the symmetry plane (Figure 3-12b) presented a zigzag pattern as a function of the $y-$ coordinate in the unnotched-beam model. This RSF was characterized by three neutral points of zero stress and two alternating regions of tensile stresses balanced by compressive stresses. The computed residual stresses by the four point bending test simulation at the peak points were $86,-57,63$ and $-107 \mathrm{MPa}$. The results of residual 
stresses along the symmetry plane in the unnotched-beam model were shown to agree well with the experimental results obtained from the slitting method for test specimens B2 and B4, as observed in Figure 3-12b. The value of the residual stresses at the peak points for the test specimen B2 were 90, $-48,55$ and $-71 \mathrm{MPa}$, and for test specimen $\mathrm{B} 4$ were $110,-53,53$ and $-62 \mathrm{MPa}$. This zigzag distribution of stresses is typical of plastically deformed beam specimens and corresponds to residual stresses of type I that self-equilibrate over a length of the same order as the specimen's dimensions according to $\int_{x_{1}}^{x_{2}} \sigma_{x} d x=0[28,81]$.

The narrow differences between the numerical and experimental results of residual stresses and strains could be attributed to minor calibration errors on either the numerical model or experimental tests or both, minor geometrical differences between machined specimens, minor misalignments during experimental tests, etc. "It is simply a fact of life that no two measurements or samples of material are identical" [10]. Also, it was not possible to prescribe both the tension and the compression material properties in the material definition employed in the FEA model (only the tension data was employed). To improve the correlation between the numerical and the experimental results, it would be necessary to implement a user material subroutine in ABAQUS ${ }^{\text {TM }}$. The user material subroutine should be capable of monitoring the first invariant of the stress tensor at the gauss points of the element and employ either tension or compression material parameters according with the sign of the hydrostatic stress tensor in the element. Nevertheless, the numerical and experimental results indicated that a well-known, consistent and repeatable RSF was introduced into the unnotched-beam specimens. 
The use of the FEA to model RSF due to inhomogeneous plastic deformation has increased over the last years. Several publications reported RSF determined by finite element simulations of inhomogeneous plastic deformation $[86,87,56,8,88,89,90]$. However, just a few $[88,90]$ presented experimental measurements for comparison with the numerical results of RSF, and the comparison revealed a poor performance of the finite element simulations. A common characteristic of these finite element models published in the literature was the use of a standard definition for the material's yield stress at $0.2 \%$ proof stress. Figure $3-13$ presents the residual stress field determined from the finite element model (Figure 3-4) with an initial yield point defined at $0.025 \%$ (397 $\mathrm{MPa})$ and $0.2 \%(466 \mathrm{MPa})$ proof stress. There was a difference of $69 \mathrm{MPa}$ between both definitions of initial yield point, which represented a difference in total strain of 0.002665 as observed in Figure 3-14. It was clear that the initial yield stress defined at $0.025 \%$ proof stress resulted in a better estimation of the RSF when comparing with the experimental measurements (Figure 3-12b). On the other hand, the initial yield stress defined at $0.2 \%$ proof stress resulted in an overestimation of the experimental determined RSF. Therefore, the definition of the initial yield point became crucial for an accurate simulation of the RSF due to four point bending test. Previous research had proved that proper characterization of the RSF was vital in order to analyze the behavior of FCG in RSF $[56,46,66]$. 


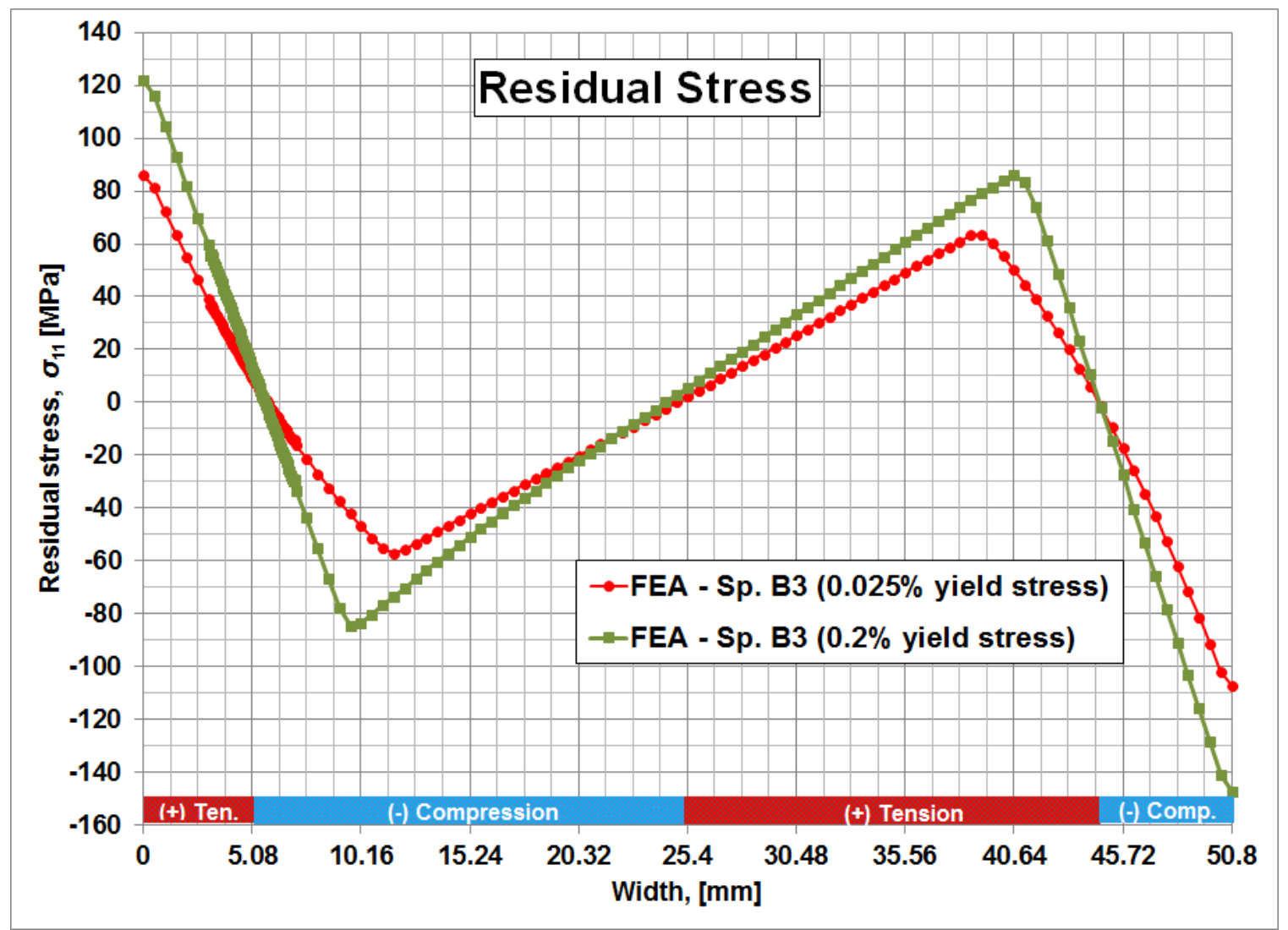

Figure 3-13 Comparison of RSF determined from the FEA model with an initial yield point at $0.025 \%$ and $0.2 \%$ proof stress. 

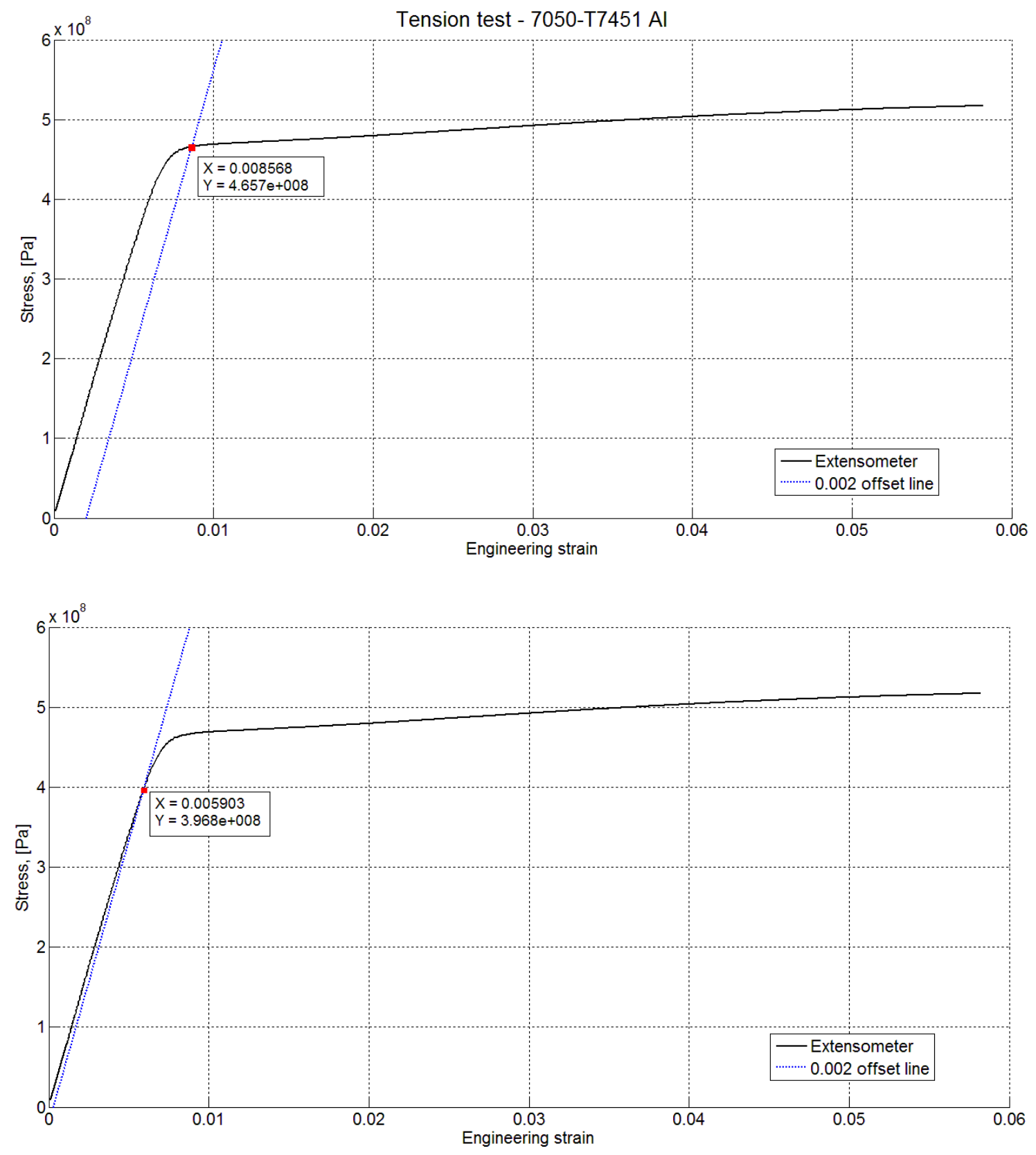

Figure 3-14 The yield stress defined at: a) $0.2 \%$ and b) $0.025 \%$ proof stress. 


\section{Chapter: Numerical Model used to analyze the Fatigue Crack Growth in Specimens with Residual Stresses}

The two traditional approaches found in the literature $[35,91,59,56,8,46]$ to analyze the FCG in RSF - elastic superposition of $K$, and $K_{\text {eff }}=K_{\max }-K_{o p}-$ do not consider the redistribution of RSF due to FCG. This manuscript presents a numerical model that used the actual RSF redistributed during the FCG. The numerical model is based on: (1) experimentally determined baseline FCG curves $(d a / d N$ vs. $\Delta K)$ for the $7050-\mathrm{T} 7451$ aluminum alloy, (2) the Harter T-method and (3) the effective stress intensity factor $K$. Baseline $d a / d N$ vs. $\Delta K$ curves for the 7050 -T7451 aluminum alloy were experimentally determined with FCG tests under constant amplitude cyclic loading for a stress ratio $R$ of $0.7,0.05$ and -1 . The Harter T-method was used to calculate new baseline $d a / d N$ vs. $\Delta K$ curves for the 7050-T7451 aluminum alloy at any stress ratio $R$ of interest. The effective stress intensity factor $K$ was used to consider the redistribution of the residual stress field due to FCG.

The experimental characterization of the FCG curves for the 7050-T7451 aluminum alloy and the Harter T-method are presented in the first section of this chapter. A developed finite element model used to simulate FCG test is also presented in the second section of this chapter. The finite element model takes into account the initial residual stress field produced by the four-point bending test, and its evolution during the FCG process. An effective stress-intensity factor $K$ was computed from the finite element results according to a displacement correlation technique. The displacement correlation technique is explained in the third section of this chapter. The fourth section presents a convergence 
analysis for the computation of the effective stress intensity factor $K$. The fifth, sixth and seventh sections of this chapter present the FCG simulation and the redistribution of RSF, according to the FEA model without the crack closure effect, the FEA model with the crack closure effect and the FEA model with the addition of the crack closure and plastic wake effects. All the FEA models took into account the redistribution of the residual stresses due to FCG. The eighth section of this chapter presents three test cases for the FCG in RSF that were analyzed by the numerical model developed in this manuscript. Finally, the last section presents the experimental study conducted to verify the FCG model.

\subsection{Fatigue crack growth model}

FCG rates were determined as a function of the effective stress intensity factor range $\Delta K$ and the effective stress ratio $R$. The definition of the effective stress intensity factor range $\Delta K$ used in this work differs from the standard definition used in the literature. According to the literature, the effective stress intensity factor range is defined by equation (8), which was presented before and is repeated below:

$$
\Delta K_{e f f}=K_{\max }-K_{o p}
$$

where $K_{\max }$ is the stress-intensity factor at the maximum applied load and $K_{o p}$ is the stress-intensity factor at the applied load that first opens the crack. Experimental measurements of the crack closure effect or finite element simulations of plasticityinduced crack closure are used to define $K_{o p}$. Contrary to this definition of the effective stress intensity factor range $\Delta K_{\text {eff }}$, this manuscript uses the finite element method to 
compute an effective $K$ as a function of the actual residual stresses during the FCG, i.e the residual stresses are not constant during the FCG and the redistribution of the residual stresses is used in the computation of the effective $K$. The effective $K$ is computed from a finite element model at the corresponding applied load and the actual residual stresses, and the effective $\Delta K$ and $R$ are defined as:

$$
\begin{gathered}
\Delta K=\text { effective }\left(K_{\max }-K_{\min }\right) \\
R=\frac{\text { effective } K_{\min }}{\text { effective } K_{\max }}
\end{gathered}
$$

where the effective $K_{\max }$ and $K_{\min }$ are computed at the maximum and minimum applied loads from a finite element model that includes the redistributed residual stress field due to FCG rather than the pre-existing RSF. FCG rates were predicted with a correlation scheme of the following form:

$$
\frac{d a}{d N}=f(\Delta K, R)
$$

and the fatigue life was predicted by:

$$
N=\int_{a_{0}}^{a_{1}} \frac{d a}{f(\Delta K, R)}
$$

Experimental FCG tests were conducted to characterize the FCG relationship presented in equation (11) for the 7050-T7451 aluminum alloy without residual stresses. The FCG test were conducted following the main recommendations of the ASTM standard E647 [25], but as in the previous chapter the actual experimental tests were conducted at Delft University of Technology (TU Delft) by Lotz as part of his M. Sc. Thesis. The next paragraphs present a brief description of the experimental characterization of the baseline $d a / d N$ vs. $\Delta K$ for $7050-T 7451$ aluminum alloy. This experimental characterization is an essential part of the FCG model developed in this study, because it characterizes the FCG 
behavior of the 7050-T7451 aluminum alloy under predominantly linear elastic conditions (small scale yielding).

The experimental FCG tests were conducted under constant amplitude load and were performed on Single Edge Notched Tension (SENT) specimens of 7050-T7451 aluminum alloy in as-machined condition. Electro-discharge machining (EDM) with a $0.1 \mathrm{~mm}$ wire was employed to introduce a starting notch of $0.5 \mathrm{~mm}$ depth into the SENT specimens, which were clamped by hydraulic grips into a servo-hydraulic fatigue machine. A sinusoidal constant load ratio with a frequency of $25 \mathrm{~Hz}$ was applied for the crack initiation and the subsequent FCG test. Only at the end of the FCG test, the frequency was reduced to $5 \mathrm{~Hz}$ to allow proper measurement of the crack length. The crack length at the front- and back-sides of the SENT specimen was monitored during the crack propagation with two digital microscope cameras. The estimated accuracy of the crack length measurement system was of $0.04 \mathrm{~mm}$. The pre-cracking was conducted till an initial crack length of $1.5 \mathrm{~mm}$. The fatigue tests were conducted at stress ratios and maximum applied loads of $R=-1$ and $P_{\max }=35 \mathrm{kN} ; R=0.05$ and $P_{\max }=35 \mathrm{kN}$; and $R=$ 0.7 and $P_{\max }=55 \mathrm{kN}$. Figure 1 presents the baseline FCG curves for the 7050-T7451 aluminum alloy, which were determined by the FCG tests. The FCG rate was computed with a polynomial regression method as described in ASTM E647 standard [25]. Further, to consider the tension and bending stresses over the ends of the SENT specimen due to the clamping effects, a solution by Ahmad et al. [92] was used to compute the stress intensity factor for the SENT specimens with clamped ends. 


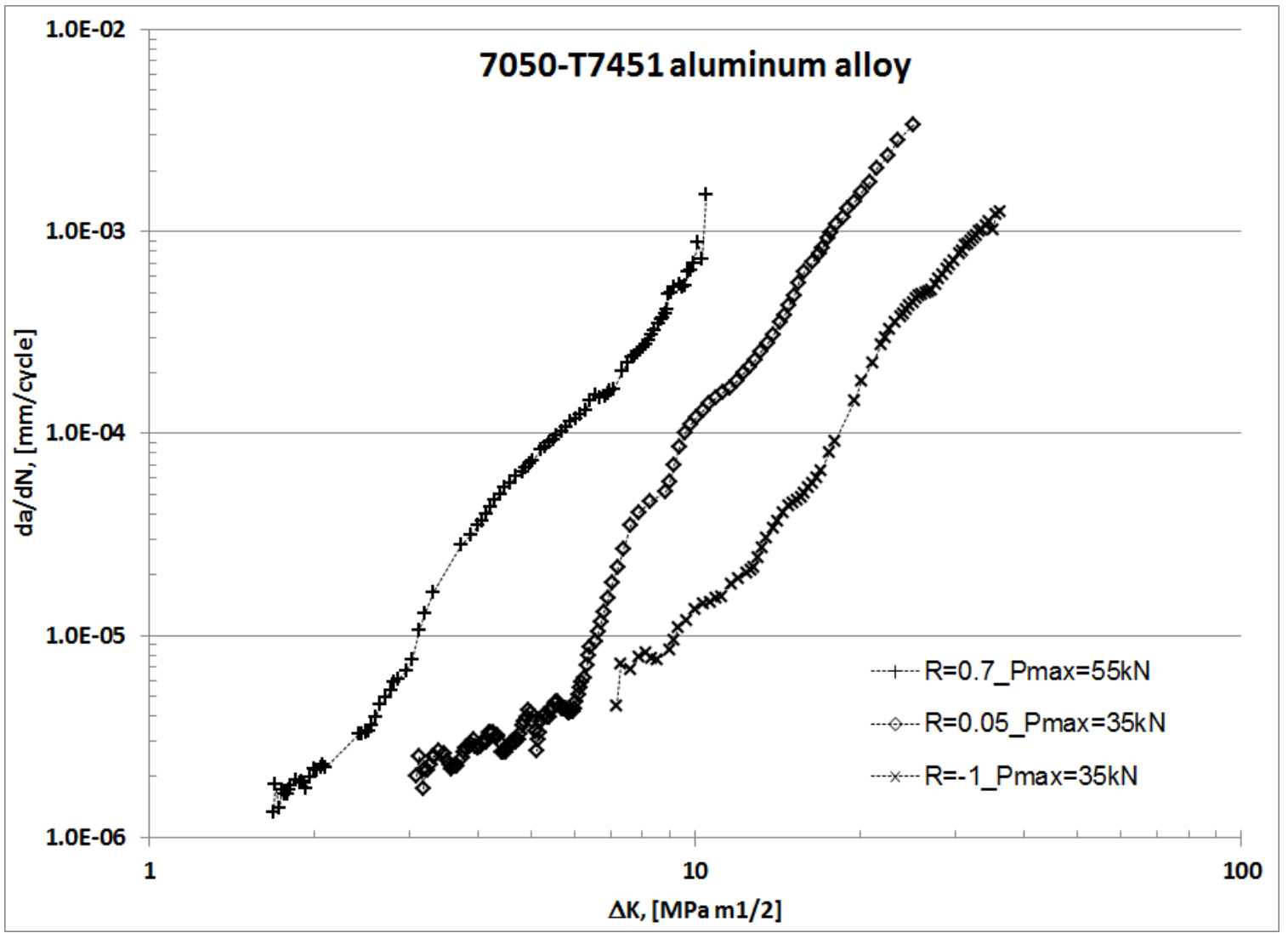

Figure 4-1 Baseline fatigue crack growth curves for 7050-T7451 aluminum alloy at $R=$ $0.7,0.05$ and -1 .

The $d a / d N$ vs. $\Delta K$ curves presented in Figure 4-1 were used as baseline material properties for 7050-T7451 aluminum alloy to predict the FCG rate in specimens with residual stresses. The FCG behavior for the linear region of logarithmic $d a / d N$ vs. $\Delta K$ curves is commonly described by the Paris-Erdogan relationship $\left(d a / d N=C \Delta K^{n}\right)$, where $C$ and $n$ are experimentally determined constants; $C$ corresponds to the intercept of the FCG curve with the ordinate and $n$ is the slope of the curve. However, the ParisErdogan relationship is used to characterize the FCG process under constant amplitude cyclic loads and small-scale yielding conditions. If residual stresses are present in the 
specimen then the Paris-Erdogan relationship should be modified to characterize the FCG process. Residual stresses in the specimens cause a variable stress ratio at the local domain of the crack tip during the FCG, in spite of the constant amplitude cycle loading condition. The FCG model developed in this study employs the Harter T-method [51] to take into account the effect of a variable stress ratio at the local domain of the crack tip during the FCG process. In addition, the Harter T-method [51] has the advantage that only a limited number of experimental baseline $d a / d N$ vs. $\Delta K$ curves are required to analyze the FCG in RSF. The Harter T-method is explained in the next section.

\subsubsection{The Harter T-Method}

The Harter T-method [51] employs an interpolation or extrapolation to determine a stress intensity factor for a stress ratio $R$ of interest as a function of the FCG rate. A minimum of two baseline FCG curves ( $d a / d N$ vs $\Delta K$ ) as the ones shown in Figure 4-1 are required to apply the Harter T-method. The Harter T-method employs the Walker equation [54], which takes into account the stress ratio $R$ in the Paris-Erdogan relationship:

$$
\frac{d a}{d N}=C\left[\frac{\Delta K}{(1-R)^{(1-m)}}\right]^{n}
$$

with two experimental FCG curves and for a specific FCG rate as observed in Figure 4-2, the equation (13) can be rewritten as:

$$
\left(\frac{d a}{d N}\right)_{i}=C\left[\frac{\Delta K_{1}}{\left(1-R_{1}\right)^{\left(1-m_{i}\right)}}\right]^{n}=C\left[\frac{\Delta K_{2}}{\left(1-R_{2}\right)^{\left(1-m_{i}\right)}}\right]^{n}
$$

this equation reduces to

$$
\frac{\Delta K_{1}}{\left(1-R_{1}\right)^{\left(1-m_{i}\right)}}=\frac{\Delta K_{2}}{\left(1-R_{2}\right)^{\left(1-m_{i}\right)}}
$$




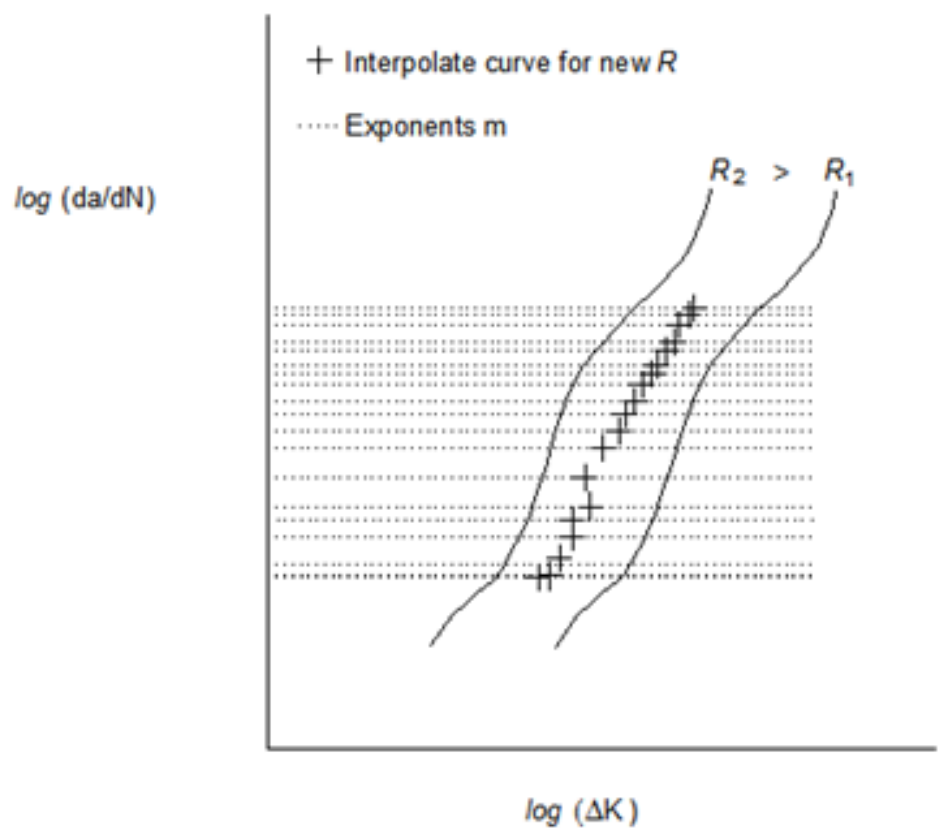

Figure 4-2 Harter T-method for interpolation of baseline $d a / d N$ vs. $\Delta K$ curves [51].

Where the empirical constants $C$ and $n$ were cancelled in equation (14), the subscripts 1 and 2 are directly linked to Figure 4-2 and the exponent $m_{i}$ is computed as a function of the specific FCG rate. Solving for $m_{i}$ :

$$
m_{i}=1+\left[\frac{\log _{10}\left(\frac{\Delta K_{1}}{\Delta K_{2}}\right)}{\log _{10}\left(\frac{1-R_{2}}{1-R_{1}}\right)}\right] \quad R_{1} \text { and } R_{2} \geq 0
$$

In the case where $R_{1}<0$ and $R_{2} \geq 0$, the maximum stress intensity factor $K_{\max }$ is used instead of the $\Delta K$ for experimental baseline $d a / d N$ vs. $\Delta K$ curves with a negative $R$; otherwise the FCG curves will not follow the proper trend, i.e. the FCG rate is higher for increasing values of the stress ratio; thus, solving for $m_{i}$ :

$$
m_{i}=1+\left[\frac{\log _{10}\left(\frac{K_{\max 1}}{\Delta K_{2}}\right)}{\log _{10}\left(\left(1-R_{1}\right)\left(1-R_{2}\right)\right)}\right] \quad R_{1}<0 \text { and } R_{2} \geq 0
$$


For a specfic FCG rate, the substitution of the exponent $m_{i}$ along with the experimental baseline $d a / d N$ vs. $\Delta K$ data point back into equation 15 allows to compute a new stress intensity factor range $\left(\Delta K_{i}\right)$ for the stress ratio of interest $\left(R_{\text {int }}\right)$ :

$$
\Delta K_{i}=\Delta K_{1}\left(\frac{1-R_{1}}{1-R_{\text {int }}}\right)^{m_{i}-1} \quad R_{1} \text { and } R_{2} \geq 0
$$

If the experimental baseline $d a / d N$ vs. $\Delta K$ curve is for a negative stress ratio then the maximum stress intensity factor $\left(K_{\max }\right)$ is computed according to:

$$
\Delta K_{\max i}=\Delta K_{2} \frac{\left(1-R_{2}\right)^{m_{i}-1}}{\left(1-R_{\text {int }}\right)^{1-m_{i}}} \quad R_{1}<0 \text { and } R_{2} \geq 0
$$

In the present FCG model, the exponent $m_{i}$ was computed at several FCG rates in the range of $1.0 \times 10^{-6}$ to $1.25 \times 10^{-3} \mathrm{~mm} /$ cycle. This range encompassed the expected fatigue crack lengths for the linear FCG regime of the $d a / d N$ vs. $\Delta K$ curve. A new FCG data point ( $\Delta K_{i}, R_{\text {int }}$ ) was found from the experimental baseline curves for each exponent $m_{\mathrm{i}}$, i.e. for each FCG rate. A linear interpolation in the $\log$-log scale was used between the new FCG data points to determine a complete new FCG curve for a stress ratio of interest. Figure 4-3 shows the data points $\left(d a / d N_{i}, \Delta K_{i}\right)$ selected from the experimental baseline FCG curves of the 7050-T7451 aluminum alloy (Figure 4-2) to compute the exponents $m_{\mathrm{i}}$, and interpolated new FCG data points for a stress ratio of interest $R_{\text {int }}$. In addition, Figure 4-3 shows baseline FCG curves that were interpolated with the Harter Tmethod for $R_{\text {int }}=0.6,0.4,0.2,-0.5$ and -0.7 . The next section describes the finite element model used to compute the effective $\Delta K$ and $R$ parameters. 


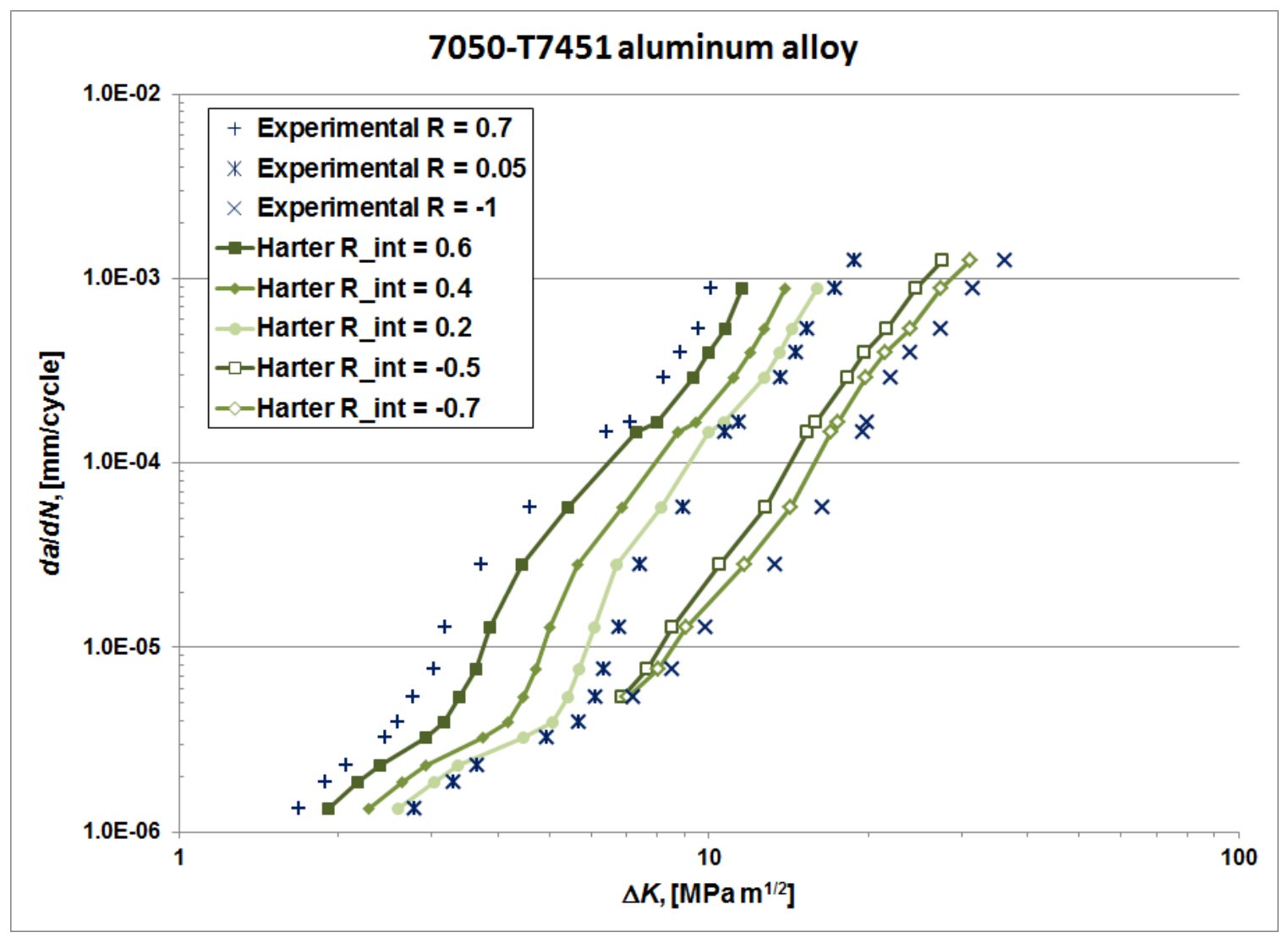

Figure 4-3 Experimental fatigue crack growth data points used to compute the exponent $m_{i}$ in the Harter T-method, and new interpolated baseline curves for $R=0.6,0.4,0.2,-0.5$ and -0.7 .

\subsection{Finite element model for the fatigue crack growth test simulation}

A Single Edge Notched Tension (SENT) specimen configuration was selected to analyze the FCG process. The SENT specimen is presented in Figure 4-4 with a schematic representation of a pre-existing RSF. The overall dimensions of the SENT specimen were identical to the finite element model of the unnotched-beam specimen used in the fourpoint bending test simulation, which was described in the previous chapter. The residual 
stress and strain fields introduced into the unnotched-beam model by the four point bending test simulation are regarded as pre-existing for the FCG test simulation. A through-thickness crack of $5.08 \mathrm{~mm}$ was introduced into the specimen's side with preexisting residual stresses in tension (upper side in Figure 4-4). The analysis assumes that the crack grows under the linear region of the $d a / d N$ vs. $\Delta K$ curve so that the FCG threshold region (fatigue crack initiation) was not considered. Furthermore, three FCG simulations were conducted and the results used to calculate the effective $K$, the effective $K$ with the crack closure effect, and the effective $K$ with the addition of the crack closure and plastic wake effects.

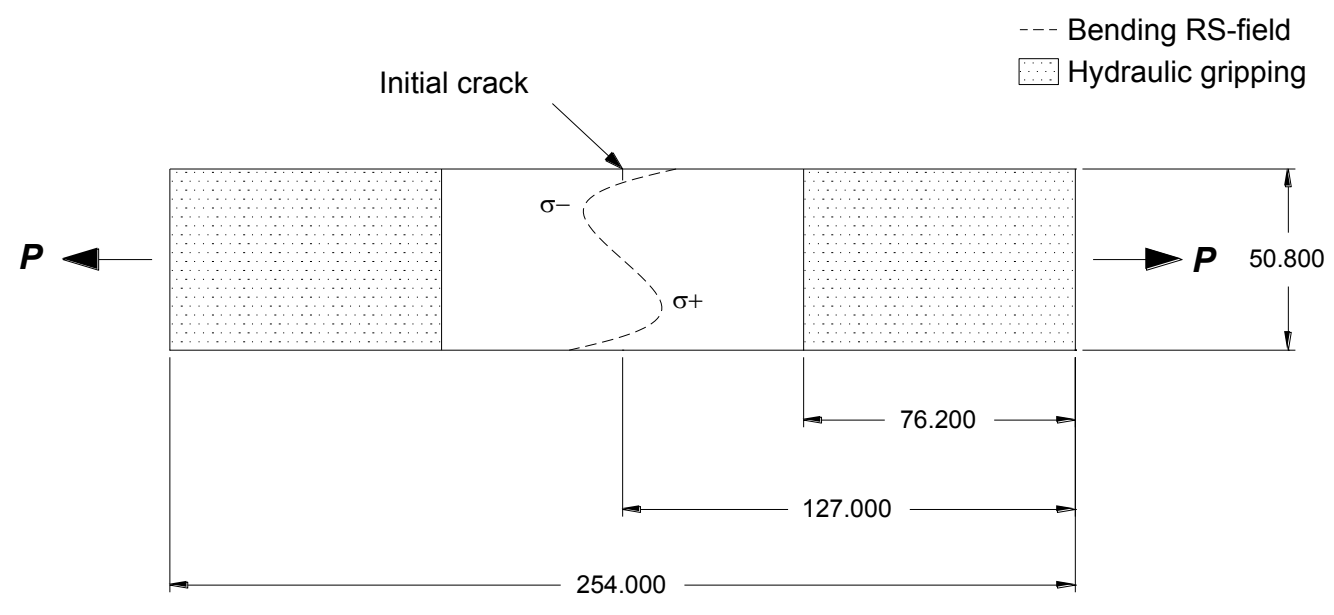

Specimen's thickness: $12.7 \mathrm{~mm}$

Dimensions: $\mathrm{mm}$

Figure 4-4 SENT specimen for the fatigue crack growth model with a schematic plot of pre-existing residual stresses produced by four-point bending.

The finite element model presented in the previous chapter was modified to simulate a FCG test under mode I. Due to symmetry conditions only half of the SENT specimen was 
modeled. Figure 4.5 presents the finite element model, which consists of a threedimensional mesh for the SENT specimen and for the grip part. In order to take into account the evolution of the pre-existing residual stresses in the SENT model, the residual stress and strain fields produced by the four point bending test simulation were used as an initial condition in the FCG test simulation. Then, the boundary conditions used during the four point bending test simulation were modified to allow the redistribution of the residual stresses during the FCG test simulation. An initial fatigue crack $a_{0}$ with a length of $5.08 \mathrm{~mm}$ was introduced into the SENT specimen's side with pre-existing residual stresses in tension. This was accomplished by releasing the fixed nodes at the symmetry plane of the unnotched-beam model for the crack length, so that the unnotched-beam model was modified into the SENT model. In addition, this scheme allowed to prescribe the plastic strain history due to four-point bending test simulation as an initial condition to the FCG test simulation. This residual plastic strain remained constant during the FCG test simulations, with the exception of the finite element model that includes the addition of the crack closure and plastic wake effects. The mesh for the SENT specimen was identical to the unnotched-beam model: a structured mesh with 8node linear brick elements with reduced integration. A total of 67,830 brick elements were used to mesh the entire SENT specimen. A focused mesh was used for the region around the crack tip, which is explained in the next section of this manuscript. In the remaining region close to the symmetry plane of the specimen (Figure 4-5b), the mesh consists of $100 \times 88$ elements with an aspect ratio of 1.117 for the plane $x-y$. The number of elements for the thickness of the SENT specimen was 5 . 


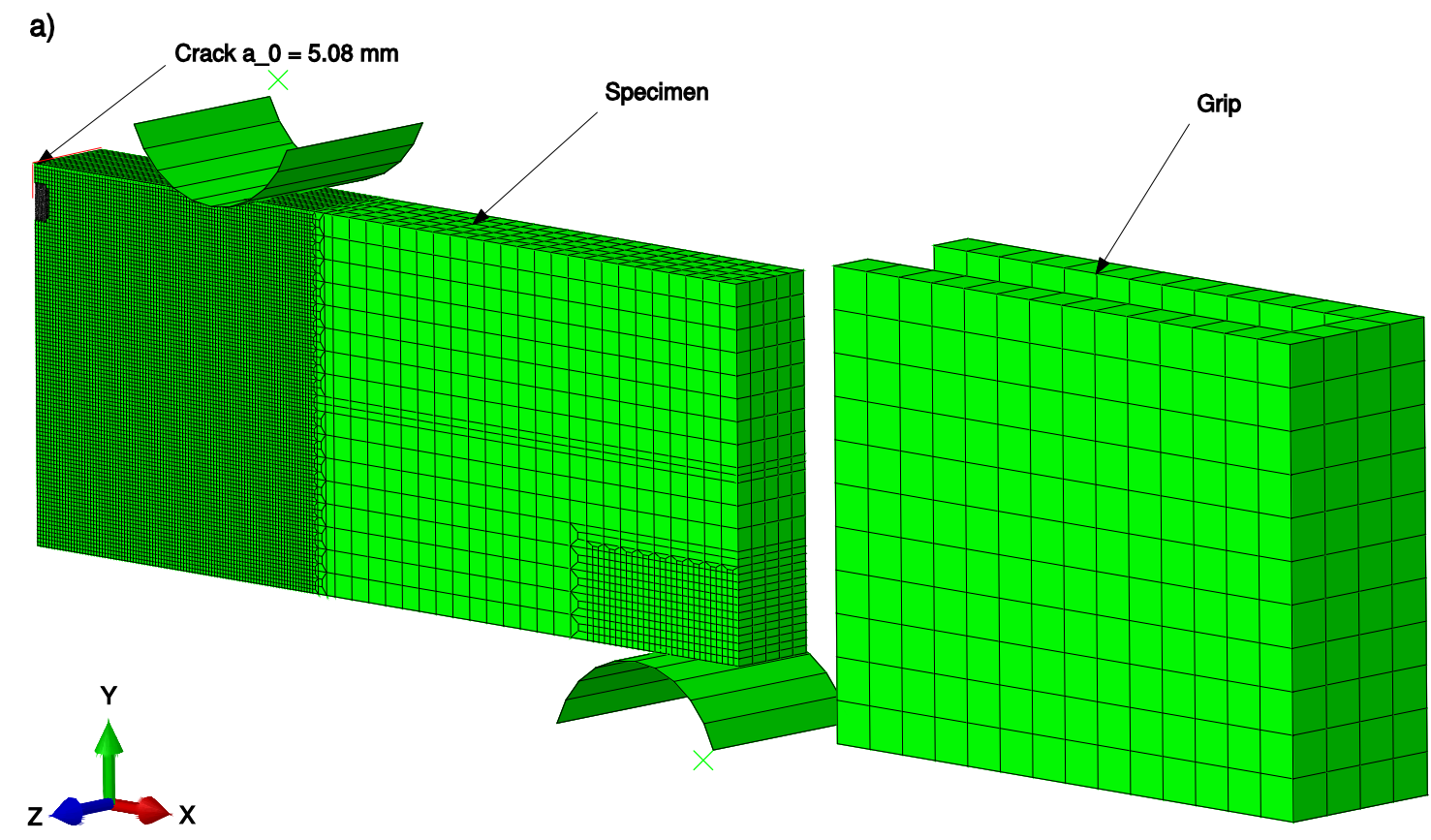

b)

Focused mesh

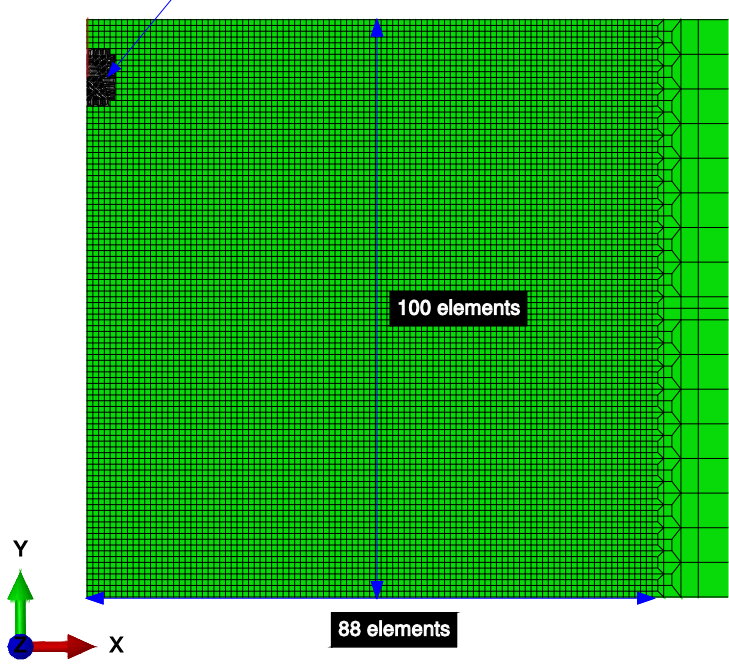

c)

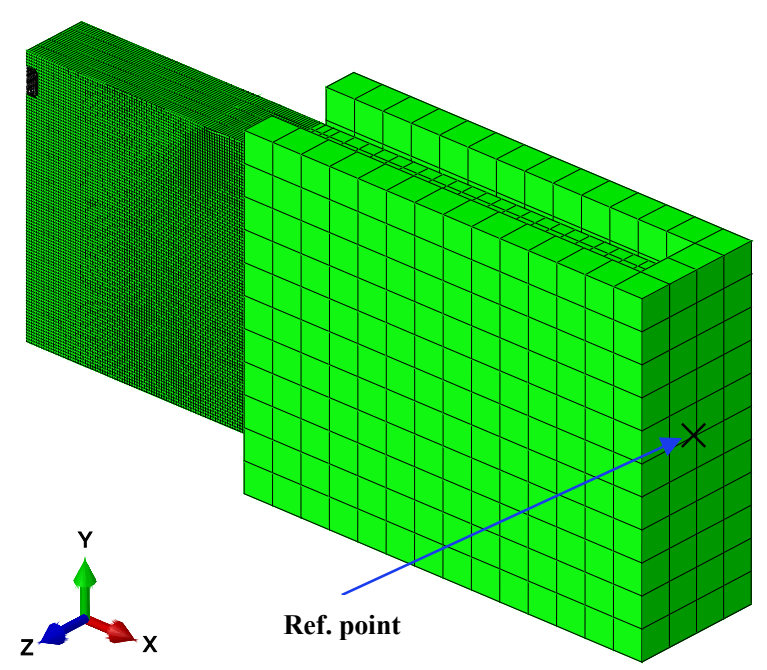

Figure 4-5 a) Half symmetry FEA model of SENT specimen and grip part for the fatigue crack growth test simulation, b) mesh detail of the specimen with a crack $a_{0}=5.08 \mathrm{~mm}$, and c) clamping of the specimen by the grip part.

The grip part was included to simulate the conditions of standard experimental FCG tests, where the test specimen is clamped by hydraulic grips. The grip was meshed with 8-node 
linear brick elements. It was assumed that the stiffness of the grip part was considerably higher than the SENT specimen; thus, a rigid body option was used for the mesh of the grip. This option uses a point of reference for the mesh of the grip that controls the degrees of freedom for the entire elements of the grip part; the grip mesh may translate and rotate, but it cannot deform with the rigid body option. Once the beam specimen was modified into the SENT specimen in the finite element model, the grip part was placed at its corresponding position (Figure 4-5c) for the FCG test simulation by prescribing a displacement into the reference point of the grip.

Contact conditions for the tangential behavior were formulated between the grip and the specimen with a penalty method. Once the contact was established during the FCG test simulation, any possible slipping between the grip and the specimen was prevented by employing the "rough" friction option in ABAQUS ${ }^{\mathrm{TM}}$ which uses an infinite coefficient of friction [93]. Only elastic slip associated with the penalty method is allowed by the "rough" friction formulation.

Contrary to the numerical models presented in the literature review, the analysis in this study considers the evolution of the RSF due to FCG and the plastic strain history. Previous finite element models presented in the literature review were limited to set the residual stresses as an initial condition without prescribing the plastic strain history [46], or the RSF are prescribed as a constant crack face pressure during the FCG analysis [59, $56]$.

The FCG tests were simulated by applying the maximum and minimum cyclic loads $\left(P_{\max }\right.$ and $\left.P_{\min }\right)$ to the reference point in the grip part (Figure 4-5c). The $P_{\max }$ and $P_{\min }$ were applied with a direction aligned with the $x$-axis (Figure 4-5) such that the FCG test 
was simulated under mode I. Boundary conditions were applied to the grip part at the point of reference to simulate standard experimental fatigue test conditions. In experimental FCG tests, the grips are just allowed to move along one axis (direction of applied load) and are fixed to zero displacements in the remaining axes and to zero rotation around the three axes. Therefore, in the finite element model the grip part was fixed to zero displacement on the remaining $y$ - and $z$-axes and to zero rotations around the three axes $(x, y$ and $z)$. The next section describes the focused mesh employed at the local domain of the crack tip and the re-meshing strategy.

\subsubsection{Focused mesh at the local domain of the crack and re-meshing strategy}

A rectangular area of $2.032 \times 4.064 \mathrm{~mm}^{2}$ around the crack tip used a refined mesh as observed in Figure 4-6a.

a)

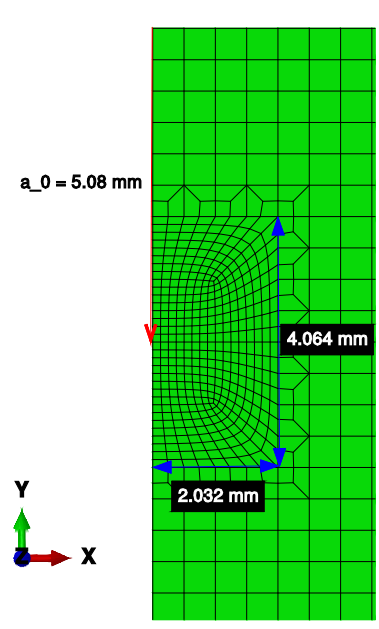

b)

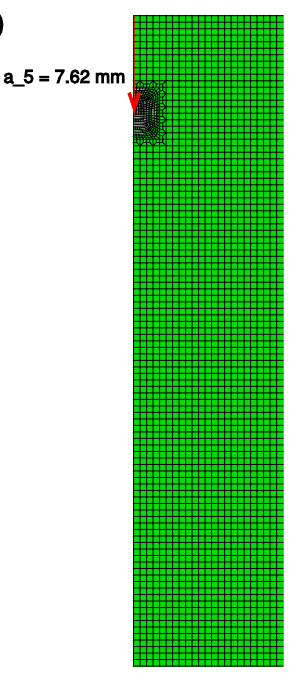

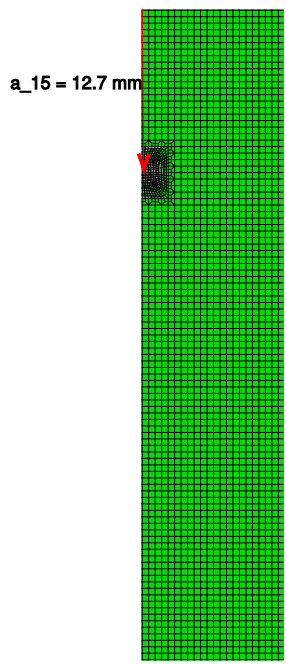

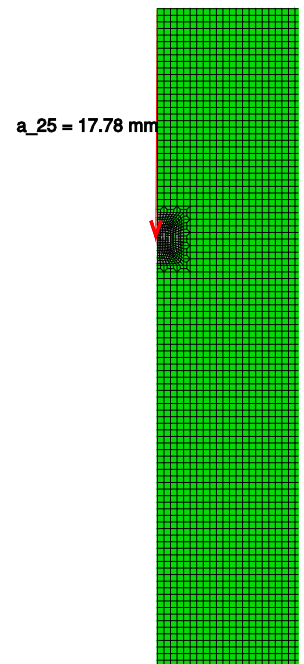

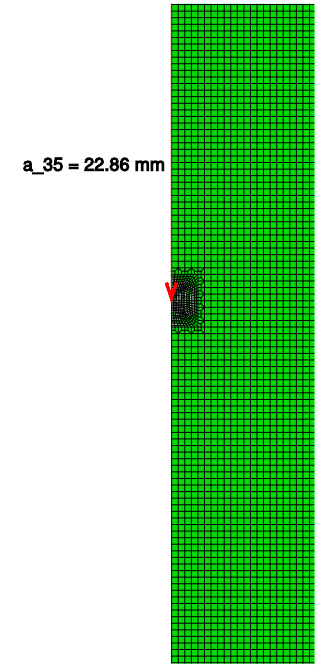

Figure 4-6 a) Focused mesh around the crack tip region, and b) re-meshed for the crack lengths $a_{5}=7.82, a_{15}=12.7, a_{25}=17.78$ and $a_{35}=22.86 \mathrm{~mm}$. 
The average element size in the refined mesh was $127 \mu \mathrm{m}$ in the plane $x-y$. The FCG was modeled by growing the crack with constant length increments $(\Delta a=0.508 \mathrm{~mm})$ from an initial crack length $a_{0}=5.08 \mathrm{~mm}$ to a final crack length of $a_{40}=25.4 \mathrm{~mm}$. The region around the crack tip was re-mesh for each fatigue crack length. Figure 4-6b shows the remeshed for fatigue crack lengths $a_{5}=7.62 \mathrm{~mm}, a_{15}=12.7 \mathrm{~mm}, a_{25}=17.78 \mathrm{~mm}$ and $a_{35}=$ $22.86 \mathrm{~mm}$.

The finite element results were used to calculate the effective stress intensity factors at $P_{\max }$ and $P_{\min }$. As previously mentioned, the unnotched-beam model was transformed into the SENT model with a pre-existing RSF so that it was not necessary to independently compute the stress intensity factor due the applied load and the RSF. Instead, the effective $K_{\max }$ and $K_{\min }$ were directly computed with the displacement correlation method from the finite element nodal displacements behind the crack tip along the crack face. The displacement correlation method is explained in the next section.

\subsection{The effective stress intensity factor calculation by the displacement correlation method}

The effective stress intensity factors $K_{\max }$ and $K_{\min }$ were calculated with a displacement correlation method. The displacement correlation method uses the finite element results of displacement from the nodes behind the crack tip to compute the effective stress intensity factor $K$. The effective $K$ was computed for each fatigue crack length at the corresponding applied loads $P_{\max }$ and $P_{\min }$. The nodes along the $y$-coordinate and behind 
the crack tip were selected for the calculation of the effective $K$ as a function of the distance to the crack tip (Figure 4-7). A plain strain condition was assumed for the local domain of the crack tip because of the $12.7 \mathrm{~mm}$ thickness of the specimen. Besides, the fracture surface of SENT specimens that were experimentally tested presented a flat surface with no shear lips, verifying the plain strain condition of the FCG. The experimental tests that were used to verify the FCG model are explained later in this manuscript. The effective stress intensity factor was calculated from the nodal displacements according to [94]:

$$
K=\frac{E u}{4\left(1-v^{2}\right)} \sqrt{\frac{2 \pi}{r}}
$$

where $E$ is the elastic modulus, $v$ is the Poisson's ratio, $r$ is the distance from the crack tip to the node position and $u$ is the displacement at the node position in the $x$-direction (Figure 4-7), which is aligned with the cyclic load.
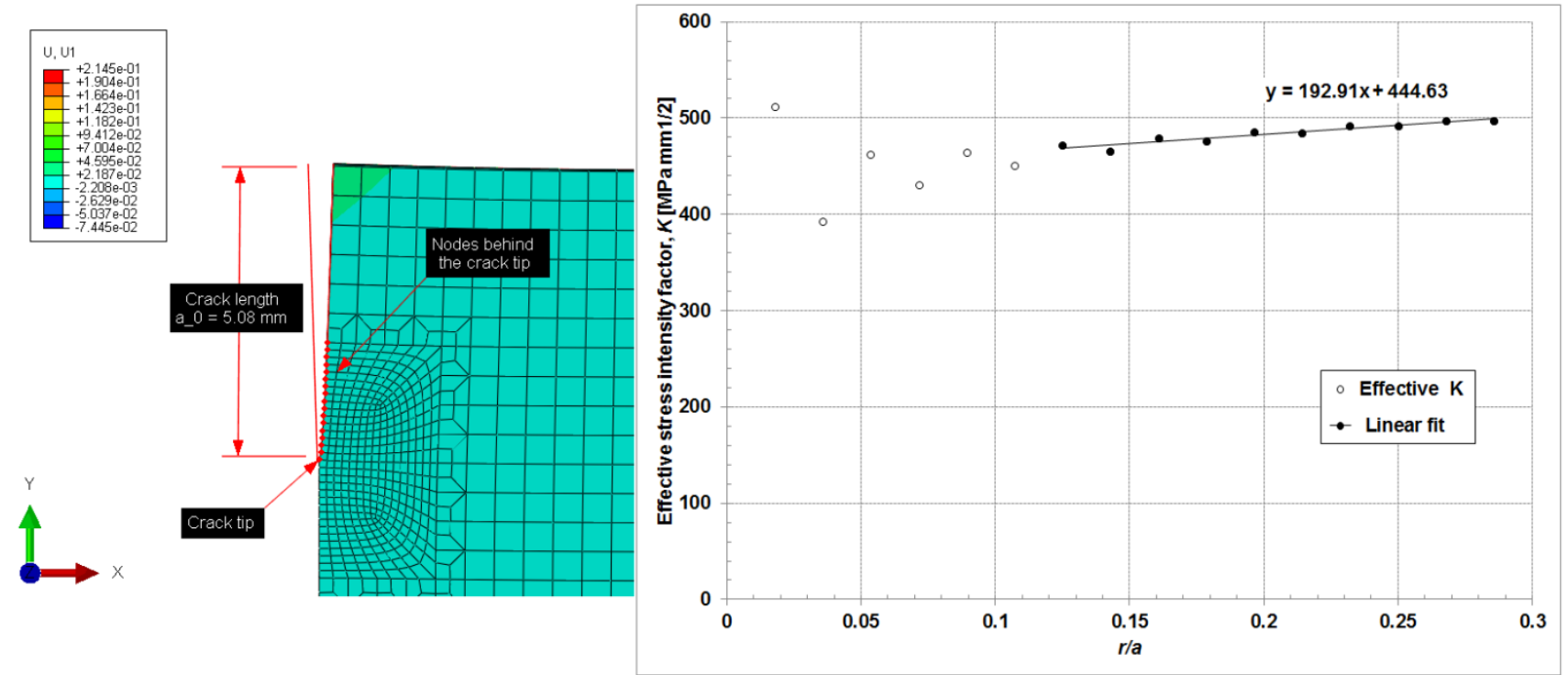

Figure 4-7 The displacement correlation technique to calculate the effective stress intensity factor. 
The effective $K$ for the nodes close to the crack tip showed an unstable behavior due to the pre-existing residual plastic strains. Disregarding the results for the first nodes close to the crack tip [94], the effective stress intensity factors $\left(K_{\max }\right.$ and $\left.K_{\min }\right)$ were found by extrapolation back to the crack tip with a linear fit of the nodes at a higher $r / a$ ratio. For instance, Figure 4-7 presents the case for the effective $K_{\max }$ at the crack length of 5.08 $\mathrm{mm}$. The specimen was subjected to the maximum nominal applied load and the effective $K_{\max }$ was computed for each node behind the crack tip within the focused mesh. The effective $K_{\max }$ was defined as the extrapolation back to the crack tip from the linear fit, i.e. the $y$-intercept of the linear fit was computed to be $\left(444.63 \mathrm{MPa} \mathrm{mm}^{1 / 2}\right)$. Due to the non-uniform residual plastic strains (four-point-bending simulation) and the evolution of the RSF as the crack grew (FCG simulation), the computed $K_{\max }$ and $K_{\min }$ were regarded as effective FCG parameters rather than just the total FCG parameters. The difference between the effective and the total $K$ is that the former considers that the pre-existing RSF redistribute for the FCG analysis, while the latter considers that the pre-existing RSF remains constant during the FCG analysis. This study only used the effective stress intensity factor, while the total one was not employed. In addition, the effects of the crack closure and the plastic wake were further considered. In total, three different FCG test simulations were conducted and the results were used in conjunction with the displacement correlation method to calculate: i) the effective $K$, ii) the effective $K$ with the crack closure effect, and iii) the effective $K$ with crack closure and plastic wake effects. 


\subsection{Mesh convergence analysis for the effective $K$ calculation}

Two element sizes were used in the FEA model to verify the mesh-independence for the effective $K$ calculation. The element sizes used for the nodes behind and around the crack tip in the SENT model were set to $0.127 \mathrm{~mm}$ and $0.056 \mathrm{~mm}$ with an aspect ratio close to one. The FEA model used the elastic-plastic material definition with combined hardening previously described in this manuscript. Figure 4-8 presents the element sizes used for the convergence analysis and a region of the mesh around the crack tip in the SENT model.

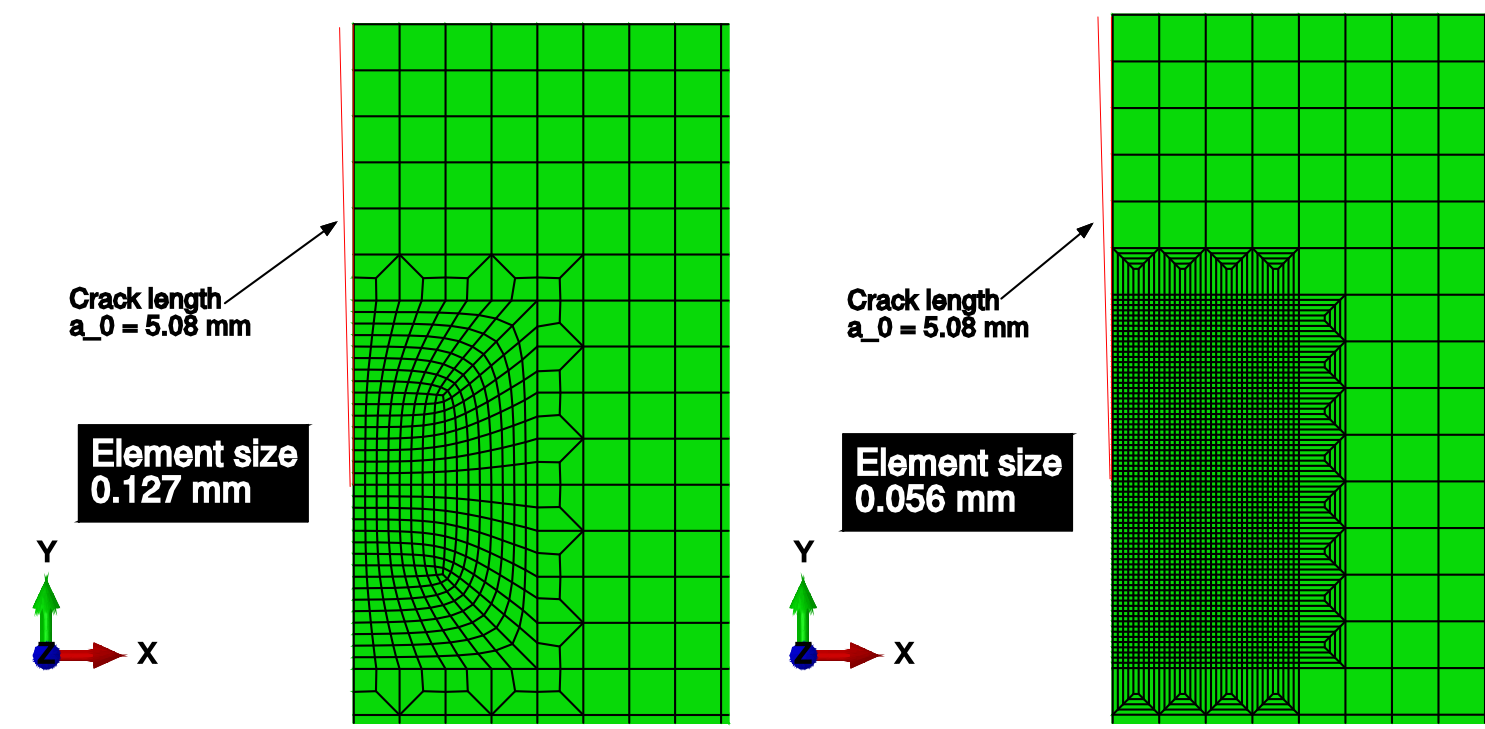

Figure 4-8 Element sizes for the mesh convergence analysis of the effective $K$ calculation: a) $0.127 \mathrm{~mm}$ and b) $0.056 \mathrm{~mm}$.

The convergence analysis was done for a crack length of $5.08 \mathrm{~mm}$, which was the initial crack length $a_{0}$ used in the FCG analysis. Pre-existing plastic strain was prescribed in the finite element model as an initial condition according to the methodology previously 
described (four-point bending simulation). In addition, the SENT model was subjected to an applied load of $35 \mathrm{kN}$, which was applied to the grip part. As previously described, the grip part was included in the finite element model to simulate the FCG test.

Figure 4-9 presents the displacement field around the crack tip obtained in the models using two different element sizes. The displacement field was similar between both element sizes of $0.127 \mathrm{~mm}$ and $0.056 \mathrm{~mm}$. However, the displacement field obtained with the element size of $0.056 \mathrm{~mm}$ produced smoother profiles.

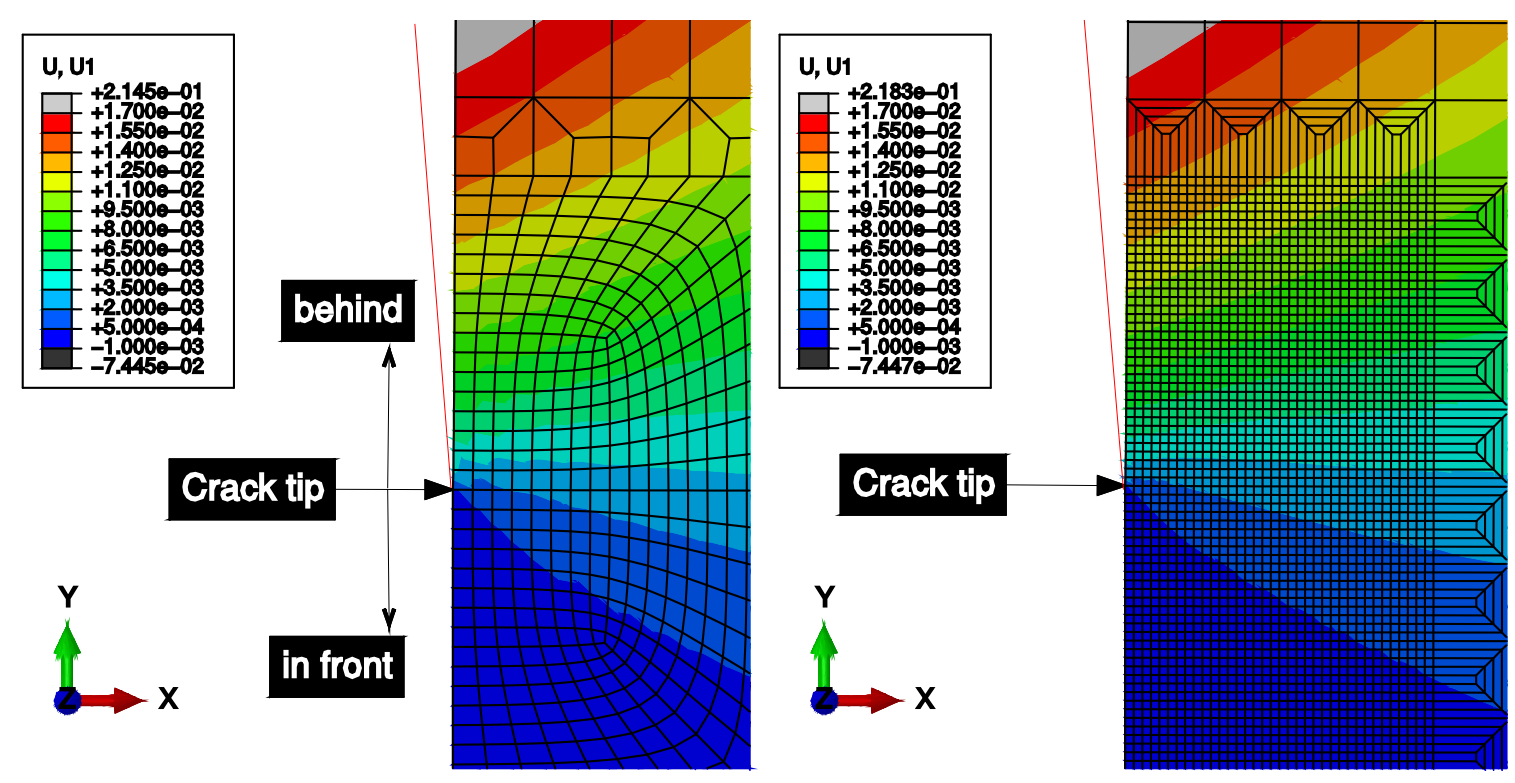

Figure 4-9 Displacement fields (mm) around the crack tip in the SENT specimen under mode I opening load: a) element size of $0.127 \mathrm{~mm}$ and b) element size of $0.056 \mathrm{~mm}$.

Figure 4-10 presents the displacement for the nodes over the crack face and behind the crack tip. These nodal displacements were used to compute the effective $K$ at the applied load of $35 \mathrm{kN}$ according to the displacement correlation technique previously described. The nodal displacements are shown as a function of the normalized distance $r / a$, where $r$ 
is the distance from the crack tip to the node position and $a$ is the crack length. The displacement along the crack face was quite similar between both element sizes $(0.127$ $\mathrm{mm}$ and $0.056 \mathrm{~mm}$ ), with the exception of the nodes close to the crack tip which show an oscillating behavior. However, even for the nodes close to the crack tip the difference was small.

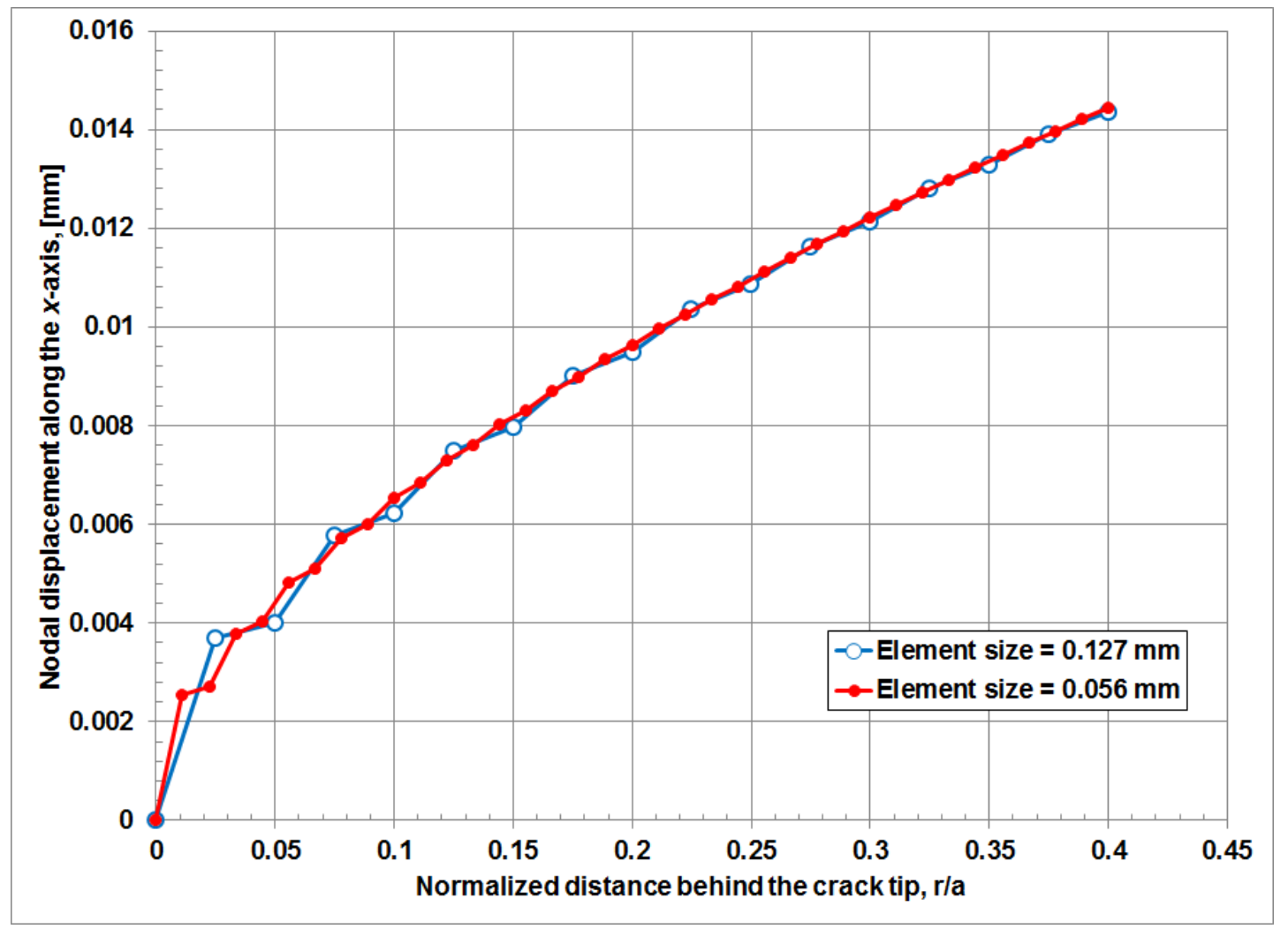

Figure 4-10 Nodal displacements behind the crack tip for element sizes of $0.127 \mathrm{~mm}$ and $0.056 \mathrm{~mm}$.

Figure 4-11 presents the effective stress-intensity factor $K$ determined with the displacement correlation technique for the two element sizes: $0.127 \mathrm{~mm}$ and $0.056 \mathrm{~mm}$. The nodes close to the crack tip were disregarded and an extrapolation back to the crack 
tip of a linear fit from the remaining nodes was used to compute the effective $K$. The effective $K$ according to both element sizes $(0.127 \mathrm{~mm}$ and $0.056 \mathrm{~mm})$ was $444 \mathrm{MPa}$ $\mathrm{mm}^{1 / 2}$ which indicate the mesh-independency in the computation of the effective $K$, i.e both element sizes produce the same value for the effective $K$.

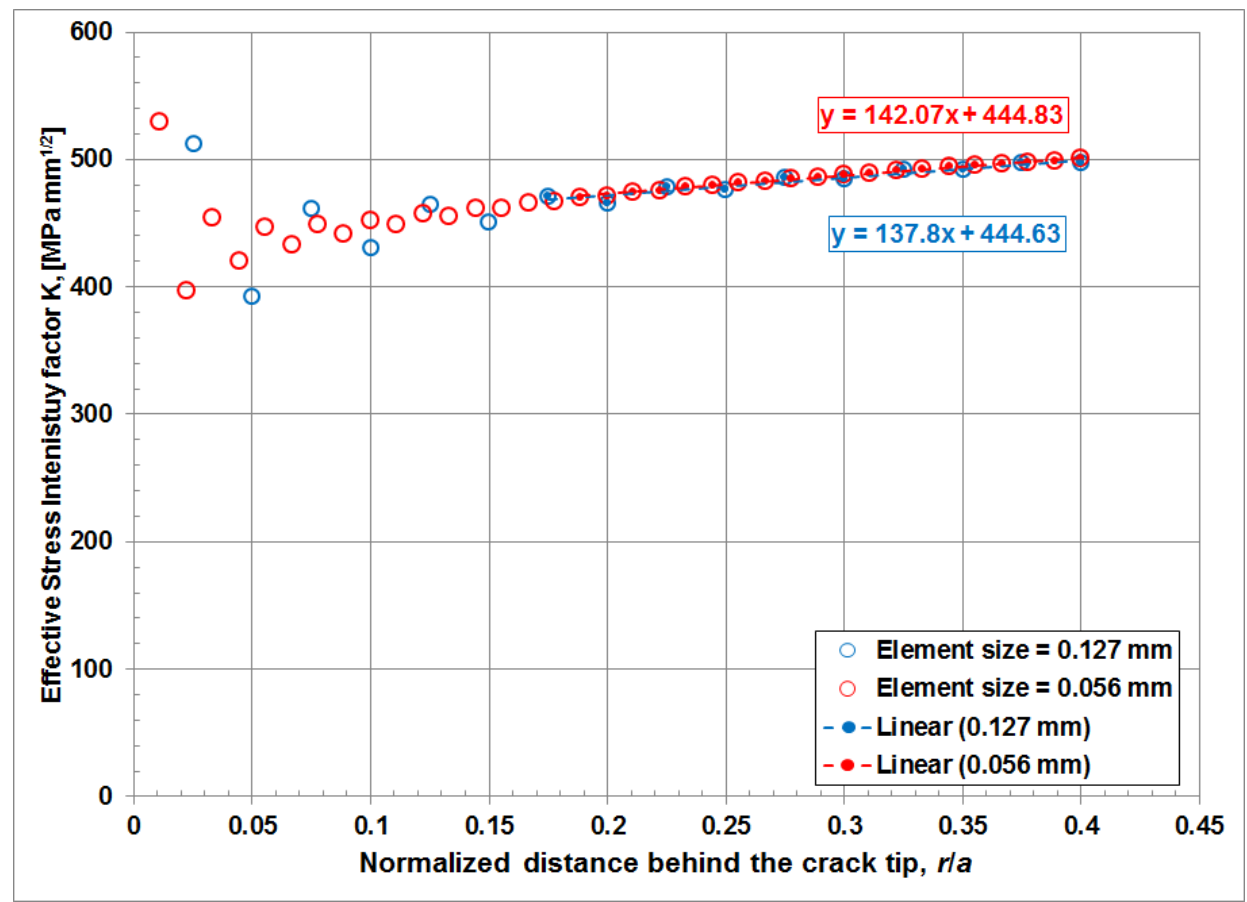

Figure 4-11 Effective stress-intensity factor $K$ according to element sizes of $0.127 \mathrm{~mm}$ and $0.056 \mathrm{~mm}$.

\subsection{Finite element model for the effective $K$ calculation without the crack closure} effect

The effective $K$ without the crack closure effect was computed with the finite element model presented in the previous section 4.2 (Figure 4-5 and Figure 4-6). A four-point bending test simulation was used to introduce a non-uniform plastic strain as an initial 
condition to the FCG test simulation. Figure 4-12 presents a flowchart of the methodology to predict the FCG rate and fatigue life. This methodology was used by the three FCG test simulations conducted in this research.

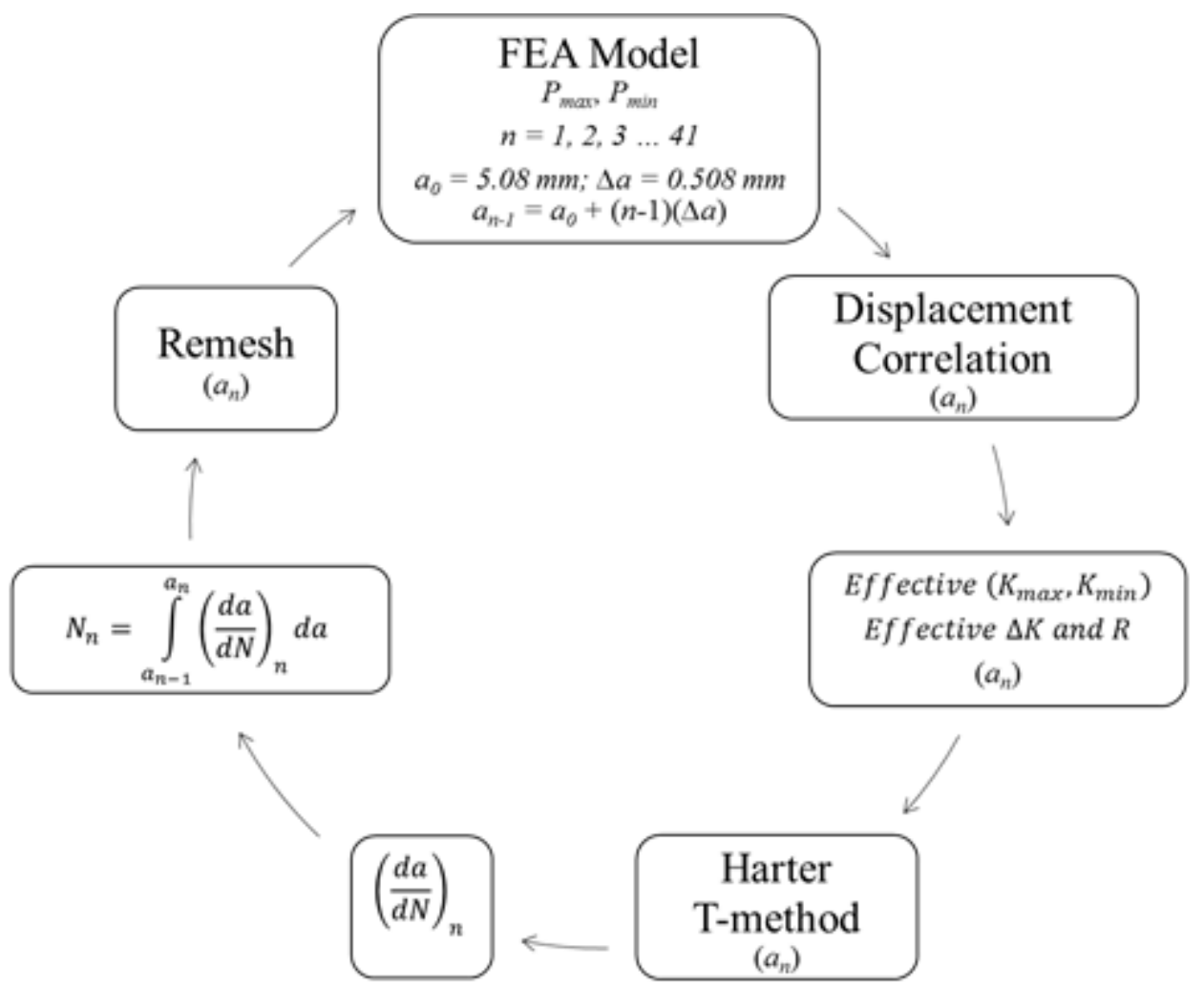

Figure 4-12 Flowchart of the FCG model to calculate the fatigue crack growth rate and fatigue life.

First, the FEA model and the displacement correlation method were used to calculate the effective $K$ for the maximum and minimum applied loads $\left(P_{\max }\right.$ and $\left.P_{\min }\right)$, as a function of the crack length $\left(a_{n}\right)$. Next, the Harter T-method was used to calculate the FCG rate as a function of the effective $\Delta K$ and $R$. Afterwards, the FCG rate was integrated over the crack length interval to calculate the number of cycles elapsed. Finally, the FEA model 
was remeshed for the next crack length and the process was repeated until the final crack length.

As previously explained, the effective $K$ was calculated at the maximum and minimum applied loads $\left(P_{\max }\right.$ and $\left.P_{\min }\right)$ and for a total of 41 fatigue crack lengths $\left(a_{0}=5.08\right.$ to $a_{40}=$ $25.4 \mathrm{~mm}$ ) with a constant crack length increment of $\Delta a=0.508 \mathrm{~mm}$. For each fatigue crack length, the effective $K$ and $R$ were used by the FCG model presented in section 4.1. The actual residual stresses were used to compute the effective $K$ rather than the preexisting RSF. The next section presents the redistribution of the RSF during the FCG test simulation.

\subsubsection{Redistribution of residual stress fields during the fatigue crack growth without the crack closure effect}

Figure 4-13 presents contour plots of the normal stress component in the $x$-direction $\left(\mathrm{S}_{11}\right.$ $\mathrm{MPa}$ ) of the SENT and unnotched-beam models without any external load applied. The unnotched-beam model was the rectangular specimen of the four-point bending test simulation (Figure 3-3) and the SENT model was the specimen of the FCG test simulation (Figure 4-4). $\mathrm{S}_{11}$ was defined as the residual stress field, since it presented the largest magnitude of stresses in the previous four point bending test simulation. Moreover, the largest component of stress was along the $x$-direction during the FCG test simulation, since it was aligned with the cyclic loading direction. The contour plots are presented for crack lengths $a_{0}=5.08 \mathrm{~mm}$ (Figure 4-13a), $a_{5}=7.62 \mathrm{~mm}$ (Figure 4-13b) and $a_{15}=12.7 \mathrm{~mm}$ (Figure 4-13c) with no external load applied, but the FCG test 
simulation included a total of 41 crack length configurations from $a_{0}=5.08 \mathrm{~mm}$ to $a_{40}=$ $25.4 \mathrm{~mm}$ with a constant crack increment $\Delta a=0.508 \mathrm{~mm}$ as previously explained. The crack was introduced at the upper side of the beam model with tensile residual stresses and its growth direction was towards the lower side of the SENT model. As a reference, an arrow indicates the crack tip position for the SENT models (Figure 4-13a, b and c), and the contour plot of the pre-existing residual stresses in the beam model is presented in Figure 4-13d.

A redistribution of the pre-existing residual stresses in the unnotched-beam model occurred when the crack was introduced and the unnotched-beam was modified into the SENT model. The pre-existing residual stresses in the beam model and the corresponding ones in the SENT model were very similar overall (Figure 4-13). The largest residual stresses in the SENT models were respectively 450.7/-307. $2 \mathrm{MPa}$ for $a_{0}=5.08 \mathrm{~mm}$ (Figure 4-13a), 450.4/-331.5 MPa for $a_{5}=7.62 \mathrm{~mm}$ (Figure 4-13b) and 450.2/-331.5 MPa for $a_{15}=12.7 \mathrm{~mm}$ (Figure 4-13c), meanwhile the corresponding residual stresses values for the unnotched-beam model were 451.2/-307.2 MPa (Figure 4-13d). However, the largest residual stresses developed in regions next to the punching marks of the four-point bending test simulation (Figure 3-3) that did not correspond to the crack position in the SENT specimen. The residual stresses of interest for the FCG test simulation were along the symmetry plane of the SENT model, which was specified as the FCG plane (Figure 4-4). The contour plots of the SENT and unnotched-beam models displayed in Figure 4-13 were limited to a stress range of 90 to $-115 \mathrm{MPa}$ to analyze the RSF of interest for the FCG test simulation. Stresses outside this range were displayed in grey color and their high magnitude was due to the local interaction between the unnotched-beam and the 
rollers during the four-point bending test simulation. No further analysis was done of residual stresses displayed in grey color.

Analyzing the region around the fatigue crack revealed a redistribution of the residual stresses in the SENT model with respect to the pre-existing state in the unnotched-beam model (Figure 4-13). The contour plots for crack lengths $a_{0}=5.08 \mathrm{~mm}$ (Figure 4-13a), $a_{5}$ $=7.62 \mathrm{~mm}$ (Figure 4-13b) and $a_{15}=12.7 \mathrm{~mm}$ (Figure 4-13c) resolved a stress concentration spot at the crack tip position, which was a consequence of the redistribution of the pre-existing RSF.
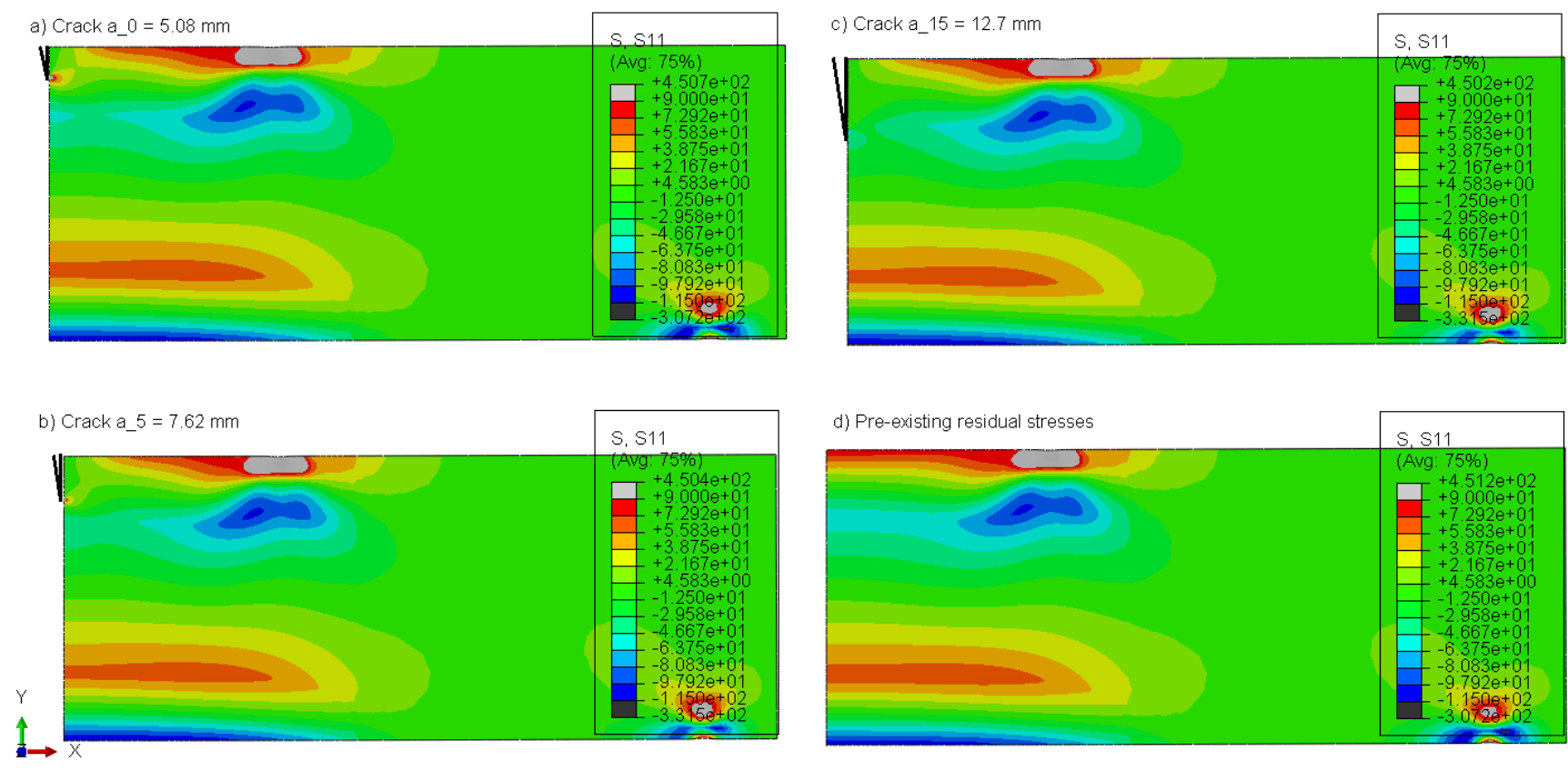

Figure 4-13 Residual stress fields ( $\mathrm{S}_{11}$ in $\mathrm{MPa}$ ) in SENT model without the crack closure effect for crack lengths: a) $a_{0}=5.08 \mathrm{~mm}$, b) $a_{5}=7.62 \mathrm{~mm}$, c) $a_{15}=12.7 \mathrm{~mm}$. d) Preexisting residual stresses in the unnotched-beam model, which was the initial condition for the fatigue crack growth test simulation. 
Figure 4-14 presents the 1-D distribution of residual stresses along the symmetry plane in the SENT model for different fatigue crack lengths. The symmetry plane was specified as the FCG plane. The residual stresses in Figure 4-14a correspond to fatigue crack lengths $a_{0}=5.08 \mathrm{~mm}, a_{5}=7.62 \mathrm{~mm}$ and $a_{10}=10.16 \mathrm{~mm}$ for the full range of $y$-coordinates in the SENT model (within the depth of the specimen). A closer view of the distribution of residual stresses for fatigue crack lengths $a_{0}=5.08 \mathrm{~mm}, a_{5}=7.62 \mathrm{~mm}$ and $a_{10}=10.16$ $\mathrm{mm}$ for a limited range from 0 to $15.24 \mathrm{~mm}$ of $y$-coordinates is plotted in Figure $4-14 \mathrm{~b}$. The plot in Figure 4-14c presents the distribution of residual stresses for fatigue crack lengths $a_{15}=12.7 \mathrm{~mm}, a_{25}=17.78 \mathrm{~mm}$ and $a_{35}=22.86 \mathrm{~mm}$ for the full range of $y$ coordinates in the SENT model. A closer view of the distribution of residual stresses for fatigue crack lengths $a_{15}=12.7 \mathrm{~mm}, a_{25}=17.78 \mathrm{~mm}$ and $a_{35}=22.86 \mathrm{~mm}$ for a limited range from 10.16 to $25.4 \mathrm{~mm}$ of $y$-coordinates is plotted in Figure 4-14d. The initial condition of the pre-existing residual stresses along the symmetry plane in the unnotchedbeam model is also plotted for comparison. 

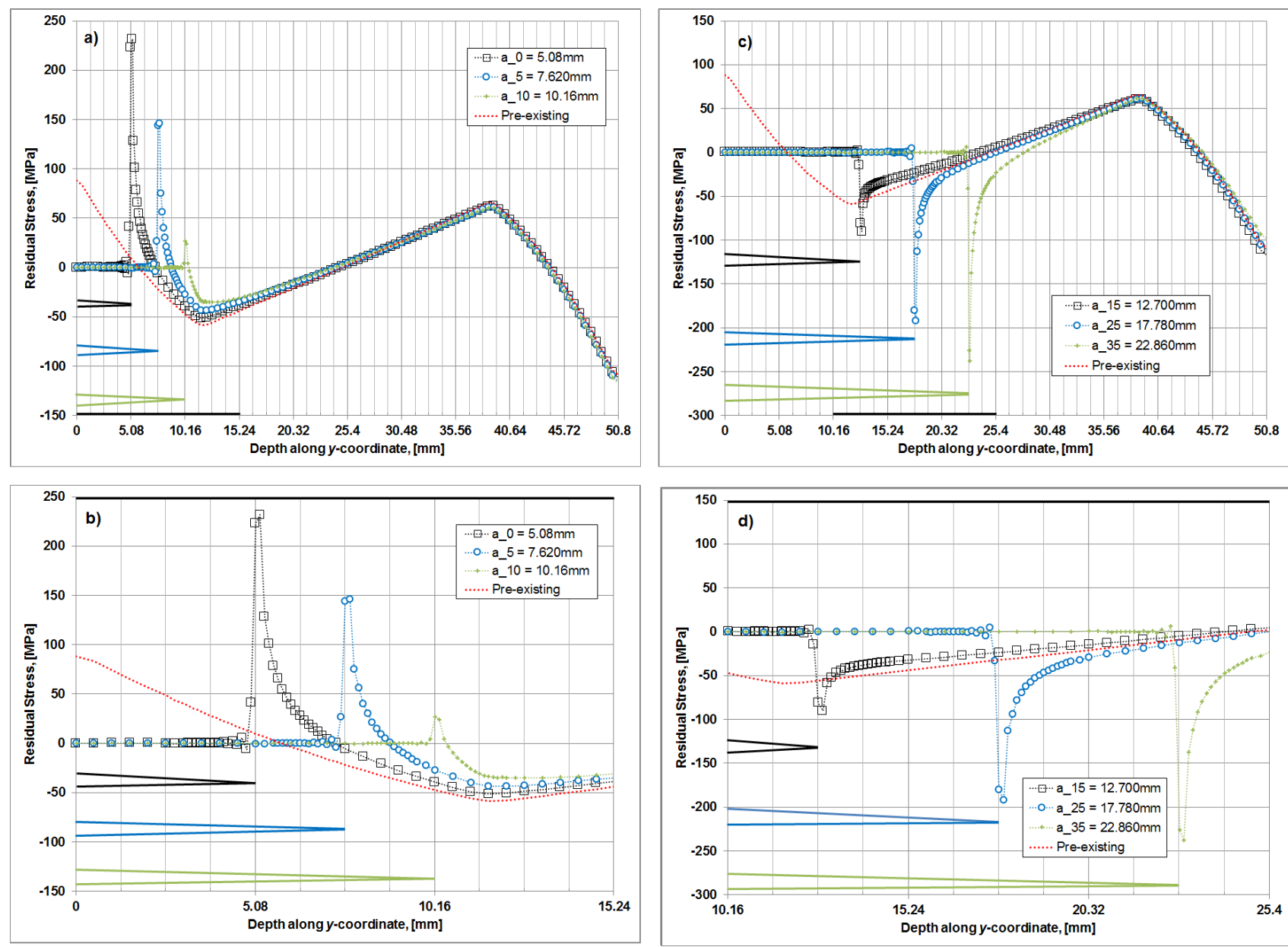

Figure 4-14 Distribution of residual stresses (1-D) along the fatigue crack growth plane in the SENT model without the crack closure effect: a) fatigue crack lengths $a_{0}=5.08 \mathrm{~mm}$, $a_{5}=7.62 \mathrm{~mm}$ and $a_{10}=10.16 \mathrm{~mm}$ with a closer view in $\mathrm{b}$ ), and c) fatigue crack lengths $a_{15}=12.7 \mathrm{~mm}, a_{25}=17.78$ and $a_{35}=22.86 \mathrm{~mm}$ with a closer view in d). Pre-existing residual stresses are also plotted for reference.

The 1-D plots indicate the redistribution of the residual stresses in regions behind and in front of the crack tip due to the FCG, which was a local phenomenon for regions around the crack tip. For the region behind the crack tip along the symmetry plane (Figure 4-14), a complete relaxation of the residual stresses (zero stress state) was observed for all the 
fatigue crack lengths analyzed. In the case of the region in front of the crack tip along the symmetry plane (Figure 4-14), an increase of the residual stresses was observed for all the fatigue crack lengths analyzed. The residual stresses at the crack tip were $225 \mathrm{MPa}$, $150 \mathrm{MPa}$ and $20 \mathrm{MPa}$ for the SENT model with fatigue crack lengths $a_{0}=5.08 \mathrm{~mm}, a_{5}=$ $7.62 \mathrm{~mm}$ and $a_{10}=10.16 \mathrm{~mm}$ (Figure 4-14b), while for fatigue crack lengths $a_{15}=12.7$ $\mathrm{mm}, a_{25}=17.78 \mathrm{~mm}$ and $a_{35}=22.86 \mathrm{~mm}$ were $-80 \mathrm{MPa},-180 \mathrm{MPa}$ and $-220 \mathrm{MPa}$ (Figure 4-14d). Figure 4-14 also presents the pre-existing residual stresses in the unnotched-beam model after the four-point bending test simulation. During the FCG test simulation, the residual stresses in front of the crack tip increased its magnitude with respect to the preexisting state and the transition from the tension state into the compressive state is a gradual process. Despite a compressive state in the pre-existing residual stresses within the range of $5.5 \mathrm{~mm}$ to $25.4 \mathrm{~mm}$ in the unnotched-beam model, the residual stresses for fatigue cracks $a_{0}, a_{5}$ and $a_{15}$ with the lengths $5.08,7.62$ and $10.16 \mathrm{~mm}$ presented a tensile state. In the case of fatigue cracks $a_{15}, a_{25}$ and $a_{35}$ with lengths $12.7,17.78$ and $22.86 \mathrm{~mm}$, the pre-existing residual stresses in compression intensified their negative values. It is these redistributions of residual stresses around the crack tip that cause a stress concentration spot at the tip of the fatigue crack as depicted in the contour plots of the SENT model (Figure 4-13).

Once the pre-existing residual stresses were redistributed due to the crack introduction, the maximum and minimum cyclic loads were applied to the SENT model during the FCG test simulation. The nodal displacements from the finite element model were employed to calculate the effective $K$. The next chapter presents the results of the 
calculations for the effective $K_{\max }, K_{\min }, \Delta K$ and $R$ for test cases 1,2 and 3, as well as the calculations for the FCG rate $(d a / d N)$ and fatigue life $(a$ vs $N)$.

\subsection{Finite element model for the effective $K$ calculation with the crack closure effect}

The crack closure effect was included in the FCG model by setting a rigid crack closure condition. The original finite element model (Figure 4-5) employed symmetry boundary conditions and just one crack face was presented; so rather than create a complete finite element model of the SENT specimen, it was decided to keep the original mesh and add an analytical rigid surface at the symmetry plane of the finite element model. The analytical rigid surface is similar to the rigid body option in that it may translate and rotate, but the surface is non-deformable. A contact interaction was defined between the crack face and the analytic rigid surface. Figure 4-15 presents the finite element model for the calculation of the effective $K$ with addition of the crack closure effect. 


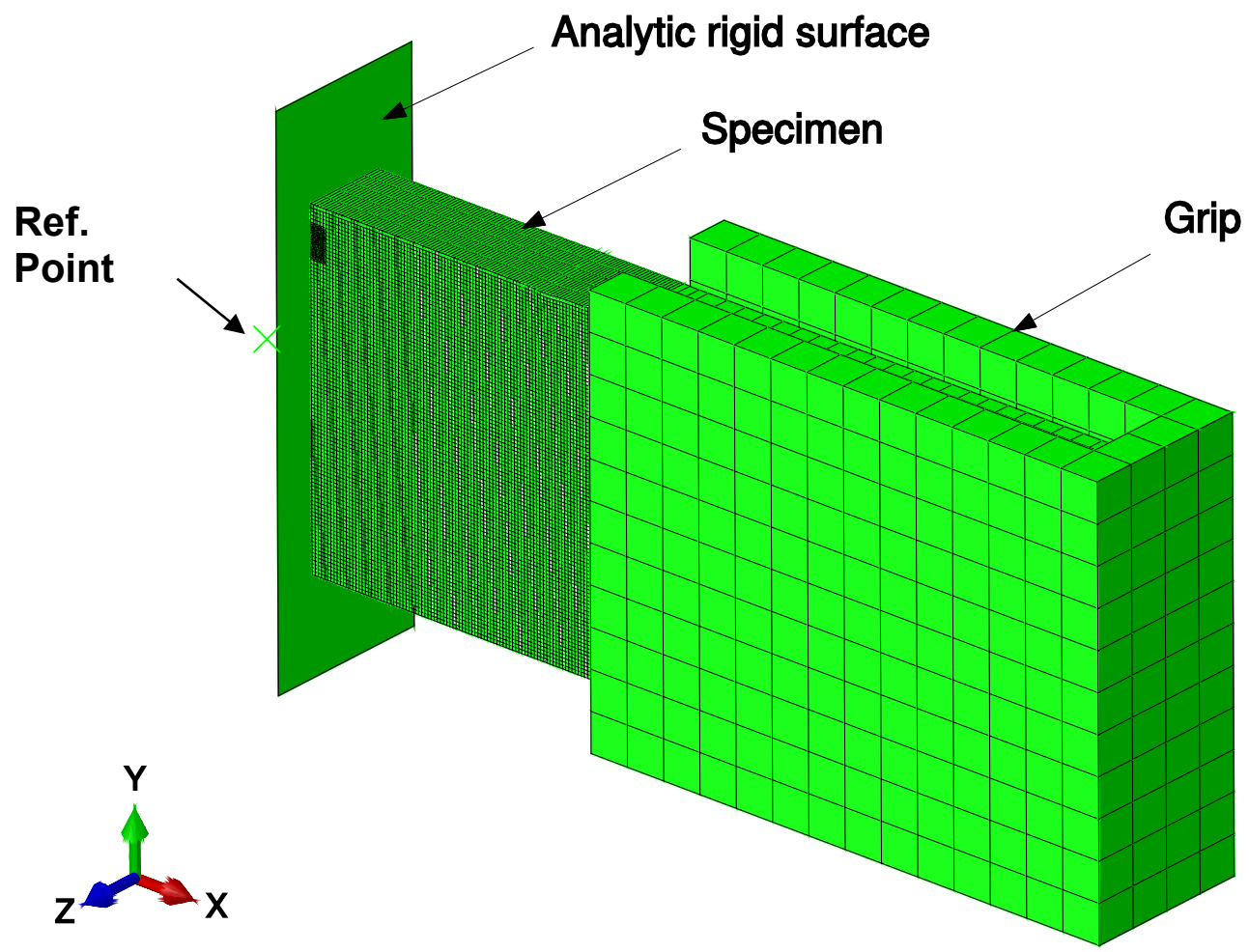

Figure 4-15 The finite element model for the effective $K$ calculation with addition of the crack closure effect.

Boundary conditions were applied to a reference point such that any possible translations or rotations of the analytical rigid surface were constrained to zero (encastre condition). The contact interaction between the analytical rigid surface and the crack face penalized negative values of the nodal displacements along the crack face at $P_{\min }$ during the FCG test simulation. As in the previous finite element model (Figure 4-5), the effective $K$ was computed at the maximum and minimum applied loads $\left(P_{\max }\right.$ and $\left.P_{\min }\right)$ for a total of 41 fatigue crack lengths $\left(a_{0}=5.08\right.$ to $\left.a_{40}=25.4 \mathrm{~mm}\right)$ with a constant crack increment $(\Delta a=$ $0.508 \mathrm{~mm}$ ), but the crack closure effect was included in this finite element model as an additional boundary condition. For each fatigue crack length, the effective $K$ and $R$ were 
employed by the FCG model presented in section 4.1. The flowchart in Figure 4-12 presents the methodology used by the FCG model.

\subsubsection{Redistribution of residual stress fields during the fatigue crack growth with the crack closure effect}

Figure 4-16 presents contour plots of the $\mathrm{S}_{11}$ in $\mathrm{MPa}$ (residual stresses component) according to the finite element model. This model includes the crack closure effect. The contour plots are presented for the SENT model with fatigue crack lengths $a_{4}=7.112 \mathrm{~mm}$ (Figure 4-16a), $a_{9}=9.652 \mathrm{~mm}$ (Figure 4-16b) and $a_{19}=14.732 \mathrm{~mm}$ (Figure 4-16c), and the pre-existing residual stresses after the four-point bending test simulation are also presented (Figure 4-16d). The pre-existing residual stresses (Figure 4-16) due to the fourpoint bending test simulation were redistributed around the crack tip as observed in Figure 4-16a, $\mathrm{b}$ and $\mathrm{c}$. The residual stresses in regions behind the crack tip for the SENT model were around a zero-stress state as indicated by the green color in the contour plots (Figure 4-16a, b and c), which contrasted with the red color in the contour plot of the unnotched beam model (Figure 4-16d). In addition, a stress concentration spot was defined at the tip of the fatigue crack. These stress concentration spots at the tip of the fatigue crack were due to the residual stresses, because no load was applied to the SENT model of Figure 4-16a, b and c. Therefore the RSF induced either the opening or the closing during the FCG as revealed by the contour plots. The contour plots depicted in Figure 4-16a and $\mathrm{b}$ for the SENT model with fatigue crack lengths $a_{4}=7.112 \mathrm{~mm}$ and $a_{9}$ $=9.652 \mathrm{~mm}$ presented a positive stress concentration spot, which indicated that the crack 
was completely open for these fatigue crack lengths. The contour plot depicted in Figure 4-16c for the SENT model with $a_{19}=14.732$ did not present a positive stress concentration spot, instead a negative region of the RSF was found around the crack tip. This indicates that the crack was partially closed due to the compressive residual stresses in the SENT model.
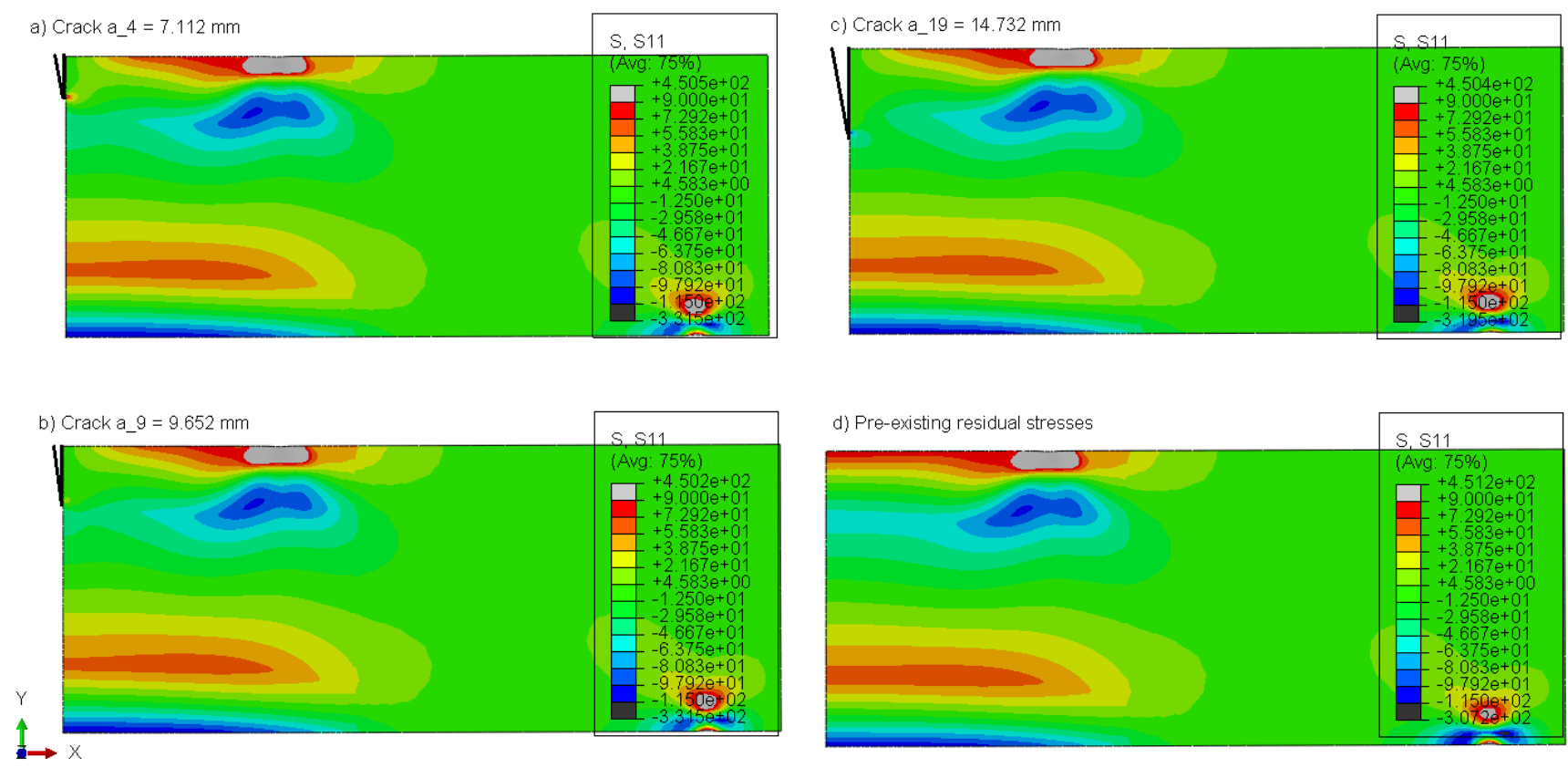

Figure 4-16 Residual stress fields $\left(\mathrm{S}_{11}\right.$ in $\left.\mathrm{MPa}\right)$ from the SENT model with the crack closure effect for fatigue crack lengths: a) $a_{4}=7.112 \mathrm{~mm}$, b) $a_{9}=9.652 \mathrm{~mm}$ and c) $a_{19}=14.732 \mathrm{~mm}$. d) Pre-existing residual stresses in the unnotched-beam model, which was the initial condition for the fatigue crack growth test simulation.

The redistribution of compressive residual stresses in the SENT model during the FCG test simulation was different between the finite element model with and without the addition of the crack closure effect. Figure 4-17 presents the redistribution of 
compressive residual stresses with and without the crack closure effect for the SENT model with the fatigue crack length $a_{25}=17.78 \mathrm{~mm}$. A complete relaxation of the residual stresses in regions behind of the crack tip for the finite element model without the crack closure effect (Figure 4-17a) was observed. The finite element model with the crack closure effect produced a region of negative residual stresses behind the crack tip (Figure 4-17b).
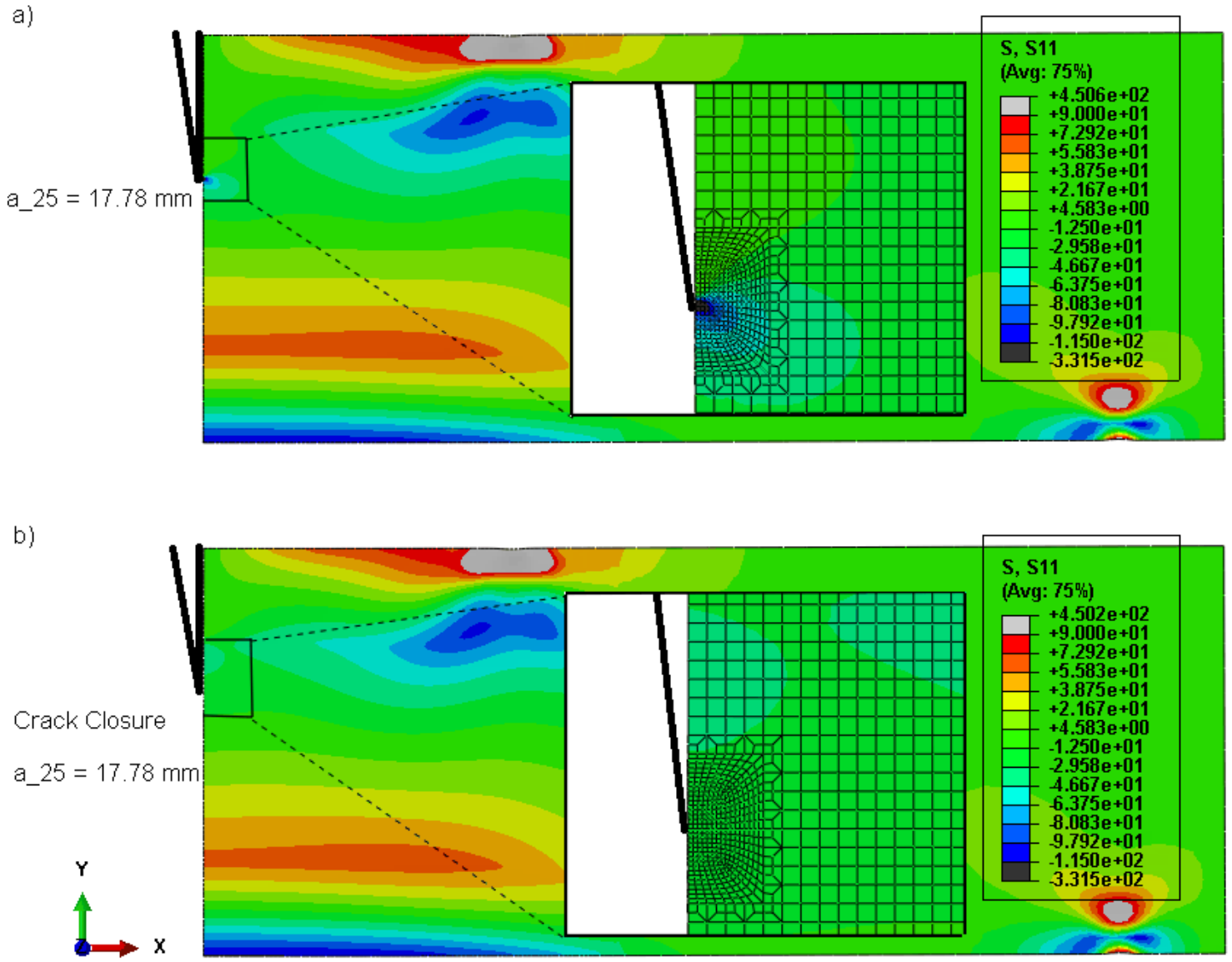

Figure 4-17 Comparison of the redistribution of residual stresses for the SENT model with fatigue crack length $a_{25}=17.78 \mathrm{~mm}$ according to: a) SENT model without the crack closure effect, and b) SENT model with the crack closure effect. 
Figure 4-18 presents the 1-D distribution of residual stresses along the symmetry plane in the SENT model with the crack closure effect for different fatigue crack lengths. Figure 4-18a presents crack lengths $a_{0}=5.08 \mathrm{~mm}, a_{5}=7.62 \mathrm{~mm}$ and $a_{10}=10.16 \mathrm{~mm}$, where the RSF were plotted for the full range of $y$-coordinates in the SENT model (within the depth of the specimen). A closer view of the distribution of residual stresses for fatigue crack lengths $a_{0}=5.08 \mathrm{~mm}, a_{5}=7.62 \mathrm{~mm}$ and $a_{10}=10.16 \mathrm{~mm}$ for a limited range from 10.16 to $25.4 \mathrm{~mm}$ of $y$-coordinates is plotted in Figure 4-18b. Figure 4-18c presents the distribution of residual stresses for fatigue crack lengths $a_{15}=12.7 \mathrm{~mm}, a_{25}=17.78 \mathrm{~mm}$ and $a_{35}=22.86 \mathrm{~mm}$ for the full range of $y$-coordinates in the SENT model. A closer view of the distribution of residual stresses for fatigue crack lengths $a_{15}=12.7 \mathrm{~mm}, a_{25}=17.78$ $\mathrm{mm}$ and $a_{35}=22.86 \mathrm{~mm}$ for a limited range from 10.16 to $25.4 \mathrm{~mm}$ of $y$-coordinates is plotted in Figure 4-18. For comparison of the residual stresses, its pre-existing state after the four-point bending simulation is also plotted in Figure 4-18. 

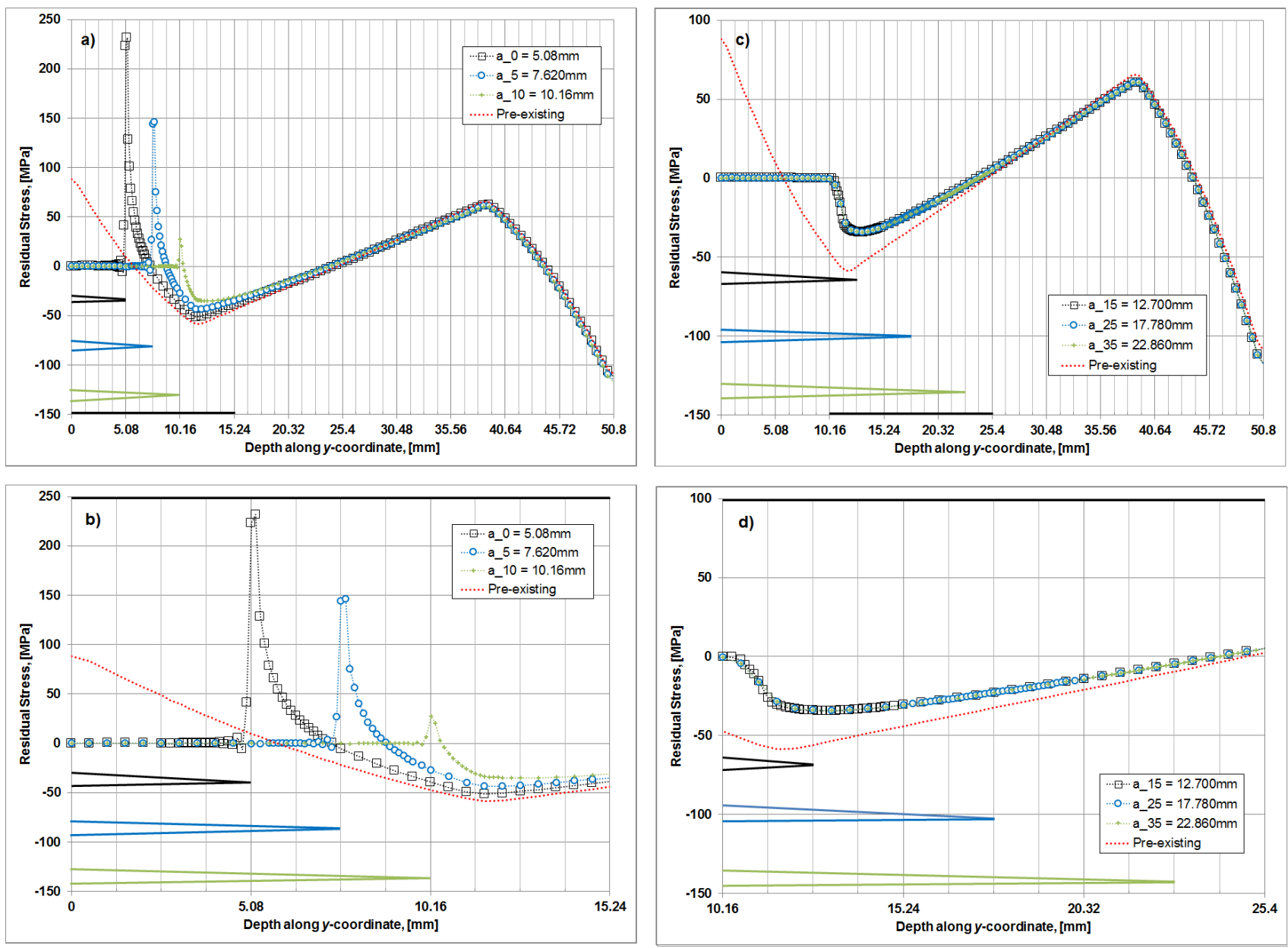

Figure 4-18 Distribution of residual stresses (1-D) along the fatigue crack growth plane in the SENT model with the crack closure effect: a) Fatigue crack lengths $a_{0}=5.08 \mathrm{~mm}, a_{5}$ $=7.62 \mathrm{~mm}$ and $a_{10}=10.16 \mathrm{~mm}$ with a closer view in b), and c) fatigue crack lengths $a_{15}=$ $12.7 \mathrm{~mm}, a_{25}=17.78$ and $a_{35}=22.86 \mathrm{~mm}$ with a closer view in d). Pre-existing residual stresses are also plotted for reference.

The redistributed residual stress at the crack tip was $225 \mathrm{MPa}, 150 \mathrm{MPa}$ and $20 \mathrm{MPa}$ for SENT model with fatigue crack lengths $a_{0}=5.08 \mathrm{~mm}, a_{5}=7.62 \mathrm{~mm}$ and $a_{10}=10.16 \mathrm{~mm}$ (Figure 4-18a and b), while for crack lengths $a_{15}=12.7 \mathrm{~mm}, a_{25}=17.78 \mathrm{~mm}$ and $a_{35}=$ $22.86 \mathrm{~mm}$ was $-34 \mathrm{MPa},-23 \mathrm{MPa}$ and $-9 \mathrm{MPa}$ (Figure 4-18c and d). The plots of residual 
stresses for crack lengths $a_{0}=5.08 \mathrm{~mm}, a_{5}=7.62 \mathrm{~mm}$ and $a_{10}=10.16 \mathrm{~mm}$ were identical to the previous finite element model without the addition of the crack closure effect (Figure 4-14a and b), which indicated that the crack closure effect was not present in the case of tensile RSF. In the case of compressive RSF, the residual stresses with the addition of the crack closure effect were different than the residual stresses without the crack closure effect. This difference was primarily produced by crack closure effect prohibiting the relaxation of the residual stresses behind the crack tip. Thus, the crack was effectively closed in the presence of compressive residual stresses and zero applied load. Figure 4-19 presents a comparison of residual stresses between the finite element model without the crack closure effect (effective $K$ ) and with the addition of it (effective $K+$ crack closure). The plots are for fatigue crack lengths $a_{15}=12.7 \mathrm{~mm}$ (Figure 4-19a), $a_{25}=17.78 \mathrm{~mm}$ (Figure 4-19b) and $a_{35}=22.86 \mathrm{~mm}$ (Figure 4-19c) and for the range of $y$ coordinates from $10.16 \mathrm{~mm}$ to $25.4 \mathrm{~mm}$ in the SENT model (within the depth of the specimen). In addition, the negative stress concentration spot that was previously found by the finite element model without the crack closure effect was also changed, thus the crack was partially closed for lengths larger than $a_{10}=10.16 \mathrm{~mm}$ according to the finite element model with the addition of the crack closure effect. 


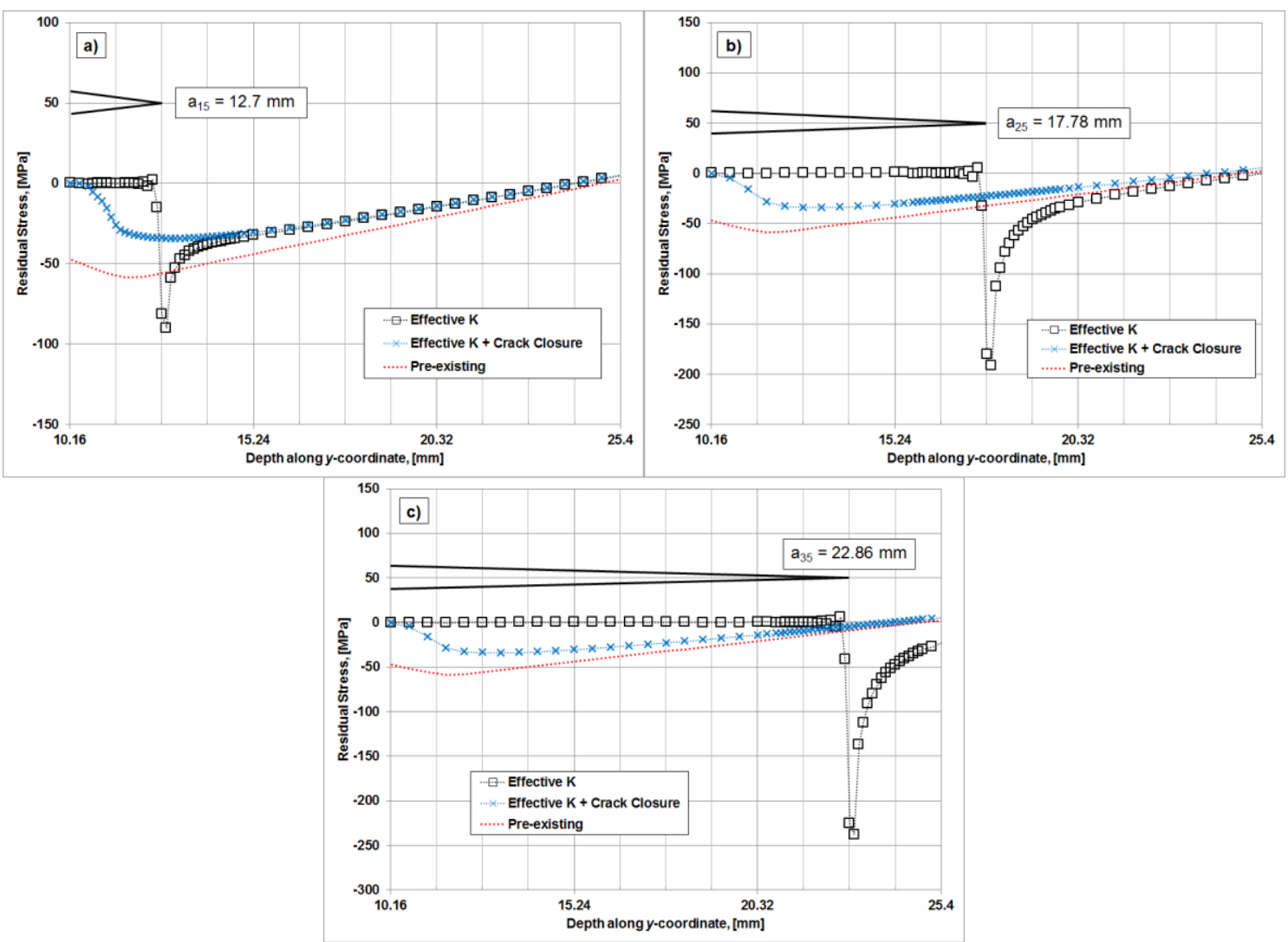

Figure 4-19 Comparison of residual stresses along the fatigue crack growth plane between the SENT models without the crack closure effect (Effective $K$ ) and with the crack closure effect (Effective $\mathrm{K}+$ Crack Closure). Crack lengths a) $\left.a_{15}=12.7 \mathrm{~mm}, \mathrm{~b}\right) a_{25}$ $=17.78 \mathrm{~mm}$ and c) $a_{35}=22.86 \mathrm{~mm}$.

\subsection{Finite element model for the effective $K$ with the addition of crack closure and plastic wake effects}

The previous finite element model (Figure 4-15) was used to conduct a plasticity-induced fatigue crack closure analysis $[43,44,45]$ so that the crack closure and plastic wake effects were included in the calculation of the effective $K$. However, the element size in the focused mesh of the finite element model (Figure 4-15) was further decreased to 
resolve the plastic deformation ahead of the crack tip. Figure 4-20 presents the focused mesh employed by the finite element model to compute the effective $K$ with addition of the crack closure and plastic wake effects.

Crack length

a_ $0=5.08 \mathrm{~mm}$
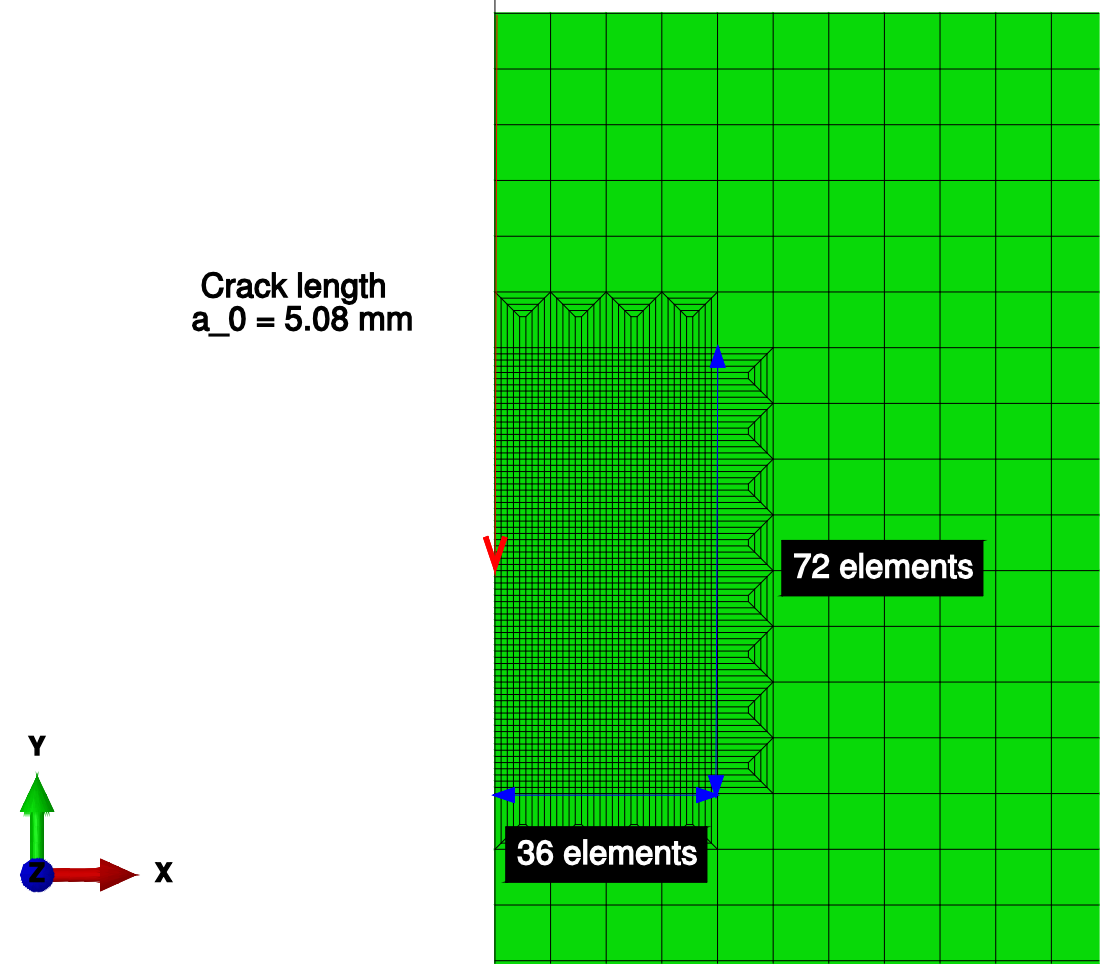

Figure 4-20 Focused mesh in the SENT model with the addition of the crack closure and plastic wake effects.

According to the Irwin plastic zone equation, the radius of the plastic zone $r_{p}$ at the crack tip is:

$$
2 r_{p}=\frac{1}{\alpha \pi}\left(\frac{K_{\max }}{\sigma_{0}}\right)^{2}
$$

where $\alpha$ is 1 and 3 for either plane stress or plane strain, $K_{\max }$ is the stress intensity factor at maximum applied load $\left(P_{\max }\right)$ and $\sigma_{0}$ is the yield stress. According to the plane stress 
condition at the free surface of the SENT specimen with a crack $\left(a_{0}=5.08 \mathrm{~mm}\right)$ and $P_{\max }$ $=35 \mathrm{kN}$, the radius of the plastic zone at the crack tip is $0.068 \mathrm{~mm}(68 \mu \mathrm{m})$. The element size employed in the previous finite element models of sections 4.6 and $4.7(127 \mu \mathrm{m})$ was higher than the radius of the plastic zone. Therefore, the element size in the focused mesh of the finite element model was decreased to an element size of $0.056 \mathrm{~mm}(56 \mu \mathrm{m})$. This ensured at least two elements in the plastic zone during the FCG test simulation at $P_{\max }$. The basic approach in the finite element analysis of the plasticity-induced fatigue crack closure is: a cyclic load is applied, the crack is advanced one element size by releasing the crack tip nodes and the contact of the crack face is monitored during the loading to define the crack opening stress, i.e. the first applied stress that opens the crack faces. The process is repeated several times to allow for the plastic wake formation. Contrary to this standard approach employed by [8], the finite element analysis in this study was not set to determine the crack opening stresses. Instead, the finite element model (Figure 4-15) with the focused mesh (Figure 4-20) at the crack tip was employed to determine the effective $K_{\max }$ and $K_{\min }$ as a function of the crack length and the FCG model developed in this study was applied. The node fixities of the boundary symmetry conditions along the crack front were released in the SENT specimen model at every $P_{\max }$ cycle load and a total of 18 cycle loads were applied to generate and stabilize the plastic wake behind the crack. As a result, the crack was advanced on an element-by-element scheme to a total length of $1.016 \mathrm{~mm}$. As with the previous analysis, a total of 41 crack length configurations were analyzed from $a_{0}=5.08 \mathrm{~mm}$ to $a_{40}=25.4 \mathrm{~mm}$ with a constant crack increment ( $\Delta a=0.508 \mathrm{~mm})$. For each crack length configuration, the effective $K$ and $R$ were employed by the FCG model presented in section 4.1. The flowchart in Figure 4-12 
presents the methodology used by the FCG model with the addition of the crack closure and plastic wake effects.

\subsubsection{Redistribution of residual stress fields during fatigue crack growth with the addition of the crack closure and plastic wake effects}

Figure 4-21 presents contour plots of $\mathrm{S}_{11}$ in $\mathrm{MPa}$ (residual stresses component) according to the finite element model with the addition of the crack closure and plastic wake effects. The contour plots are only presented for a few fatigue crack lengths $\left(a_{6}=8.128 \mathrm{~mm}\right.$, Figure 4-21a; $a_{16}=13.208 \mathrm{~mm}$, Figure 4-21b; and $a_{26}=18.288 \mathrm{~mm}$, Figure 4-21c) of a total of 41 fatigue crack length configurations analyzed ( $a_{0}=5.08 \mathrm{~mm}$ to $\left.a_{40}=25.4 \mathrm{~mm}\right)$ with a constant crack increment $\Delta a=0.508 \mathrm{~mm}$. The pre-existing residual stresses after the four-point bending test simulation are also shown in Figure 4-21d (unnotched-beam model). 

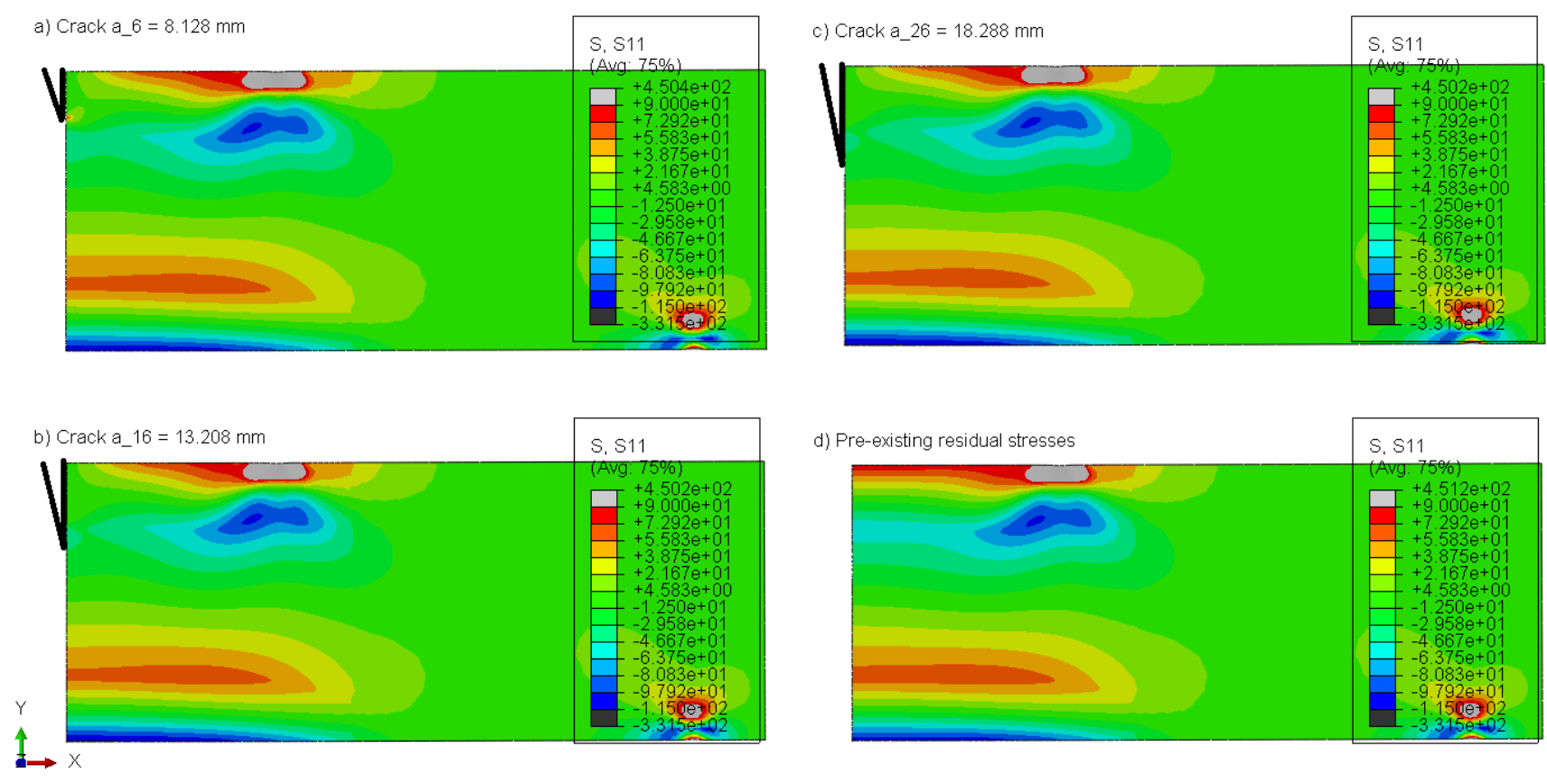

Figure 4-21 Residual stress fields from the SENT model with the addition of the crack closure and plastic wake effects for fatigue crack lengths: a) $\left.a_{6}=8.128 \mathrm{~mm}, \mathrm{~b}\right) a_{16}=$ $13.208 \mathrm{~mm}$ and c) $a_{26}=18.288 \mathrm{~mm}$. d) Pre-existing residual stresses after the four-point bending test simulation (unnotched-beam model).

For residual stresses in tension, a stress concentration spot was defined for the SENT model as observed in Figure 4-21a with the fatigue crack length $a_{6}=8.128 \mathrm{~mm}$. In the case of the SENT models with the fatigue crack lengths $a_{16}=13.208 \mathrm{~mm}$ and $a_{26}=$ $18.288 \mathrm{~mm}$, the regions around the crack tip did not present a well-defined stress concentration spot, but rather a compressive residual stress field was found. Therefore, for residual stresses in compression, a stress concentration spot was not defined and the profile of the residual stresses was similar to the pre-existing profile after the four-point bending test simulation. For a better visualization of the residual stresses profile and stress concentration spots, Figure 4-22 presents the 1-D distribution of residual stresses 
along the FCG plane in the SENT model. Again, just a few a fatigue crack lengths are presented, but the analysis was conducted for a total of 41 fatigue crack lengths with a constant crack increment of $\Delta a=0.508 \mathrm{~mm}$. Figure 4-22a presents fatigue crack lengths $a_{0}=5.08 \mathrm{~mm}, a_{5}=7.62 \mathrm{~mm}$ and $a_{10}=10.16 \mathrm{~mm}$, where the residual stresses were plotted for the complete range of $y$-coordinates in the SENT model (within the depth of the specimen). A closer view for the range of $y$-coordinate from 0 to $15.24 \mathrm{~mm}$ in the SENT model is plotted in Figure 4-22b for the residual stresses in fatigue crack $a_{0}, a_{5}$ and $a_{10}$. Figure 4-22c presents fatigue crack lengths $a_{15}=12.7 \mathrm{~mm}, a_{25}=17.78 \mathrm{~mm}$ and $a_{35}=22.86$ $\mathrm{mm}$, where again the residual stresses were plotted for the full range of $y$-coordinate in the SENT model (within the depth of the specimen). Figure 4-22d is a closer view of the residual stresses for the range of $y$-coordinate from 10.16 to $25.4 \mathrm{~mm}$ in the SENT model with the fatigue crack $a_{15}, a_{25}$ and $a_{35}$. The pre-existing residual stresses after the fourpoint bending test simulation along the specified FCG plane are also plotted as a reference of the redistribution of the residual stresses as a function of the fatigue crack length. 

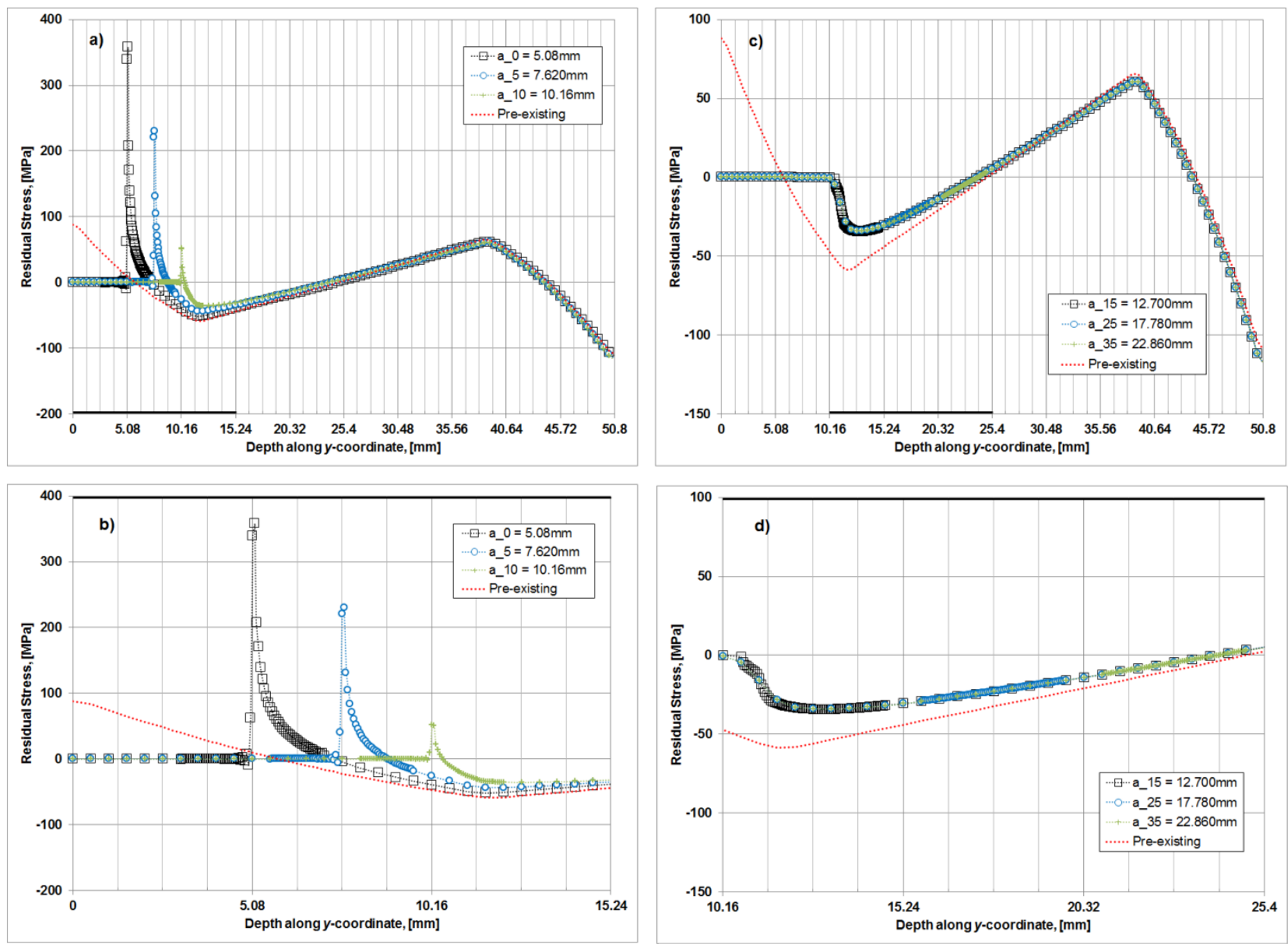

Figure 4-22 Distribution of residual stresses (1-D) along the fatigue crack growth plane in the SENT model with the incorporation of the crack closure and plastic wake effect: a) Crack length configurations $a_{0}=5.08 \mathrm{~mm}, a_{5}=7.62 \mathrm{~mm}$ and $a_{10}=10.16 \mathrm{~mm}$ with a closer view in b), and c) crack length configurations $a_{15}=12.7 \mathrm{~mm}, a_{25}=17.78$ and $a_{35}=$ $22.86 \mathrm{~mm}$ with a closer view in d). Pre-existing residual stresses after the four-point bending test simulation.

The plots of residual stresses along the FCG plane in the SENT model confirmed that the redistribution of the residual stresses due to crack introduction was a local phenomenon around the crack tip. A complete relaxation of the residual stresses was observed in 
regions behind the crack tip for crack length configurations $a_{0}, a_{5}$ and $a_{10}$ (Figure 4-22a and b). However, the residual stresses in SENT models with cracks $a_{15}, a_{25}$ and $a_{35}$ did not relax completely, and instead a compressive residual stress field was found in the region behind the crack tip. Therefore, the crack was partially closed for lengths larger than $a_{10}=$ $10.16 \mathrm{~mm}$. The redistributed residual stress at the crack tip was $340 \mathrm{MPa}, 221 \mathrm{MPa}$ and $52 \mathrm{MPa}$ for SENT models with crack lengths $a_{0}=5.08 \mathrm{~mm}, a_{5}=7.62 \mathrm{~mm}$ and $a_{10}=10.16$ $\mathrm{mm}$ (Figure 4-22b), while for crack lengths $a_{15}=12.7 \mathrm{~mm}, a_{25}=17.78 \mathrm{~mm}$ and $a_{35}=$ $22.86 \mathrm{~mm}$ was -30 MPa, -24 MPa and -6 MPa (Figure 4-22d). These conditions of redistributed residual stresses around the crack tip were the reasons for the stress concentration spot depicted in the contour plot of SENT model with crack length $a_{6}$ (Figure 4-21a), and for the lack of a stress concentration spot at cracks $a_{16}$ and $a_{26}$ (Figure $4-21 b$ and $c)$.

Figure 4-23 presents a comparison of the RSF between the actual finite element model with the addition of the crack closure and plastic wake effects, and the previous one with just the addition of the crack closure effect. In addition, the pre-existing residual stresses after the four-point bending test are also plotted. The plots are for the fatigue crack lengths $a_{0}=5.08 \mathrm{~mm}$ (Figure 4-23a), $a_{5}=7.62 \mathrm{~mm}$ (Figure 4-23b) and $a_{10}=10.16 \mathrm{~mm}$ (Figure 4-23c) for the range of $y$-coordinate from 0 to $15.24 \mathrm{~mm}$ (within the depth of the specimen), and for the fatigue crack length $a_{15}=12.7 \mathrm{~mm}$ for the range of $y$-coordinate from $10.16 \mathrm{~mm}$ to $25.4 \mathrm{~mm}$ (Figure 4-19d). 

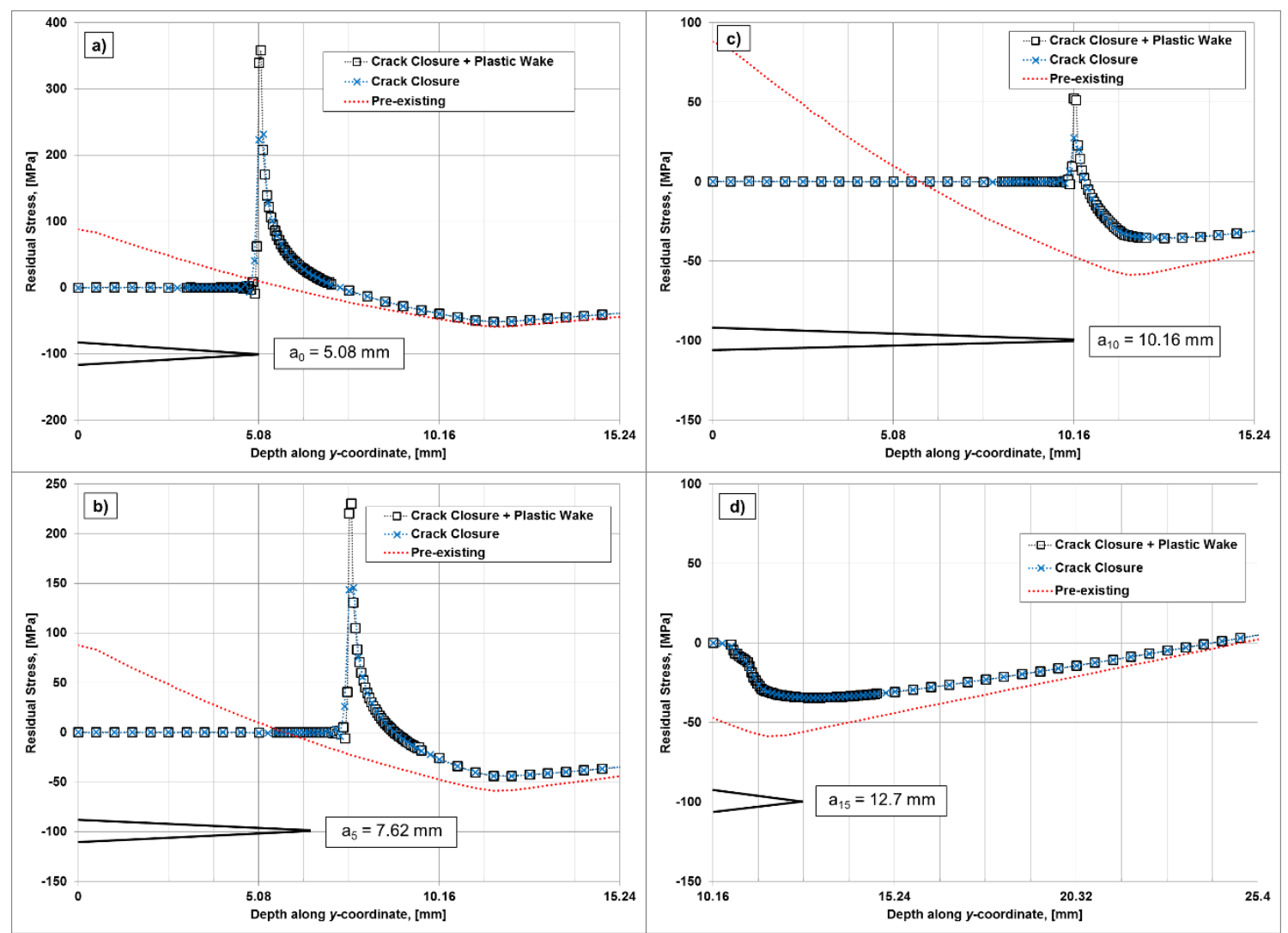

Figure 4-23 Comparison of residual stresses along the fatigue crack growth plane in the SENT model (zero external loads) between the SENT model with the addition of the crack closure and plastic wake effects, and the SENT model with only crack closure effect. Fatigue crack lengths a) $\left.a_{0}=5.08 \mathrm{~mm}, \mathrm{~b}\right) a_{5}=7.62 \mathrm{~mm}$, c) $a_{10}=10.16 \mathrm{~mm}$ and d) $a_{15}=12.7 \mathrm{~mm}$.

The finite element model with the addition of the crack closure and plastic wake effects presented larger values of residual stresses at the crack tip, as observed in Figure 4-23 for the fatigue crack $a_{0}, a_{5}$ and $a_{10}$, than the finite element model with just the addition of the crack closure effect. This difference was due to the element size employed around the crack tip region that was of $56 \mu \mathrm{m}$ for the finite element model with the addition of the crack closure and plastic wake effects, while the element size was $127 \mu \mathrm{m}$ for the finite 
element model with just the addition of the crack closure effect (Figure 4-24). In the case of fatigue crack length $a_{15}=12.7 \mathrm{~mm}$, the redistribution of the residual stress field due to crack introduction was the same between the finite element model with the addition of the crack closure and plastic wake effects, and the corresponding one with just the addition of the crack closure effect. The plots in Figure 4-23 also indicated that the overall stress field distribution was the same between the mesh with an element size of 56 $\mu \mathrm{m}($ Crack Closure + Plastic Wake) and the mesh with and element size of $127 \mu \mathrm{m}$ (Crack Closure). However, the shorter element size in the finite element model with the addition of the crack closure and plastic wake effects was able to resolve the plastic zone at the crack tip and the plastic wake developed during the FCG around the crack tip.

a)

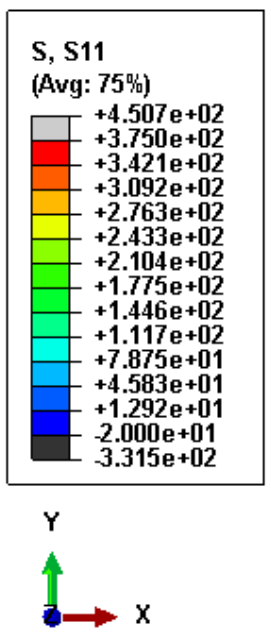

b)

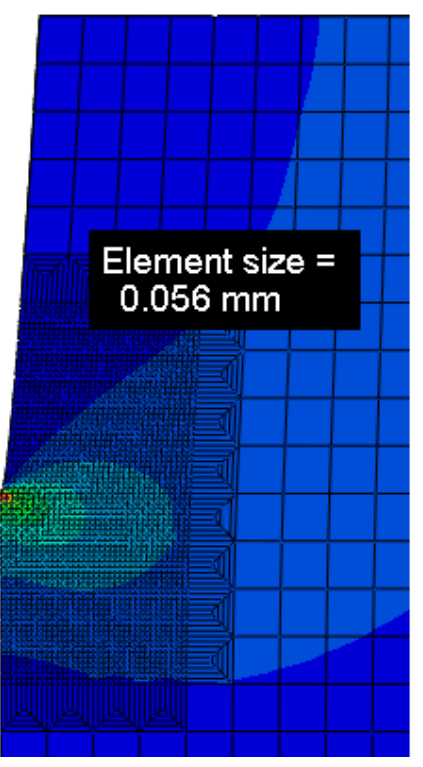

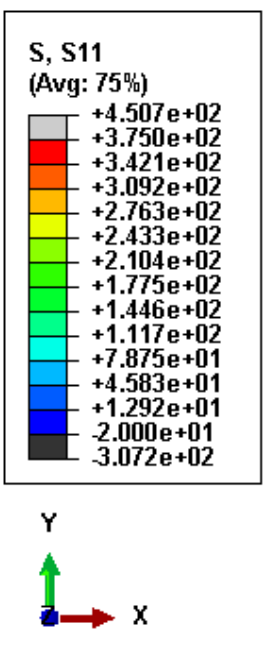

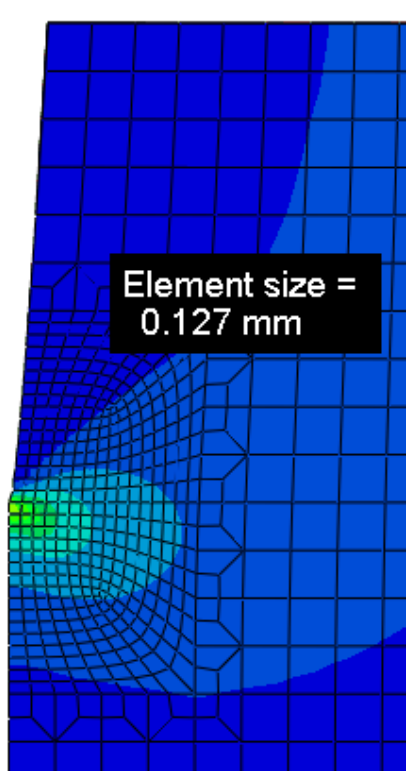

Figure 4-24 Redistribution of residual stress fields (zero external loads) for SENT model with crack length $a_{0}=5.08 \mathrm{~mm}$ as a function of the element size around the crack tip:
a) $56 \mu \mathrm{m}$ and b) $127 \mu \mathrm{m}$. 


\subsection{Test cases for the fatigue crack growth model}

Three test cases were modeled to analyze the FCG process in the SENT specimen containing residual stresses. The FCG test simulation for the first test case with a large stress ratio $R$ used $P_{\max }=55 \mathrm{kN}$ and nominal $R=0.7$, for the second case with a low stress ratio $R$ used $P_{\max }=35 \mathrm{kN}$ and nominal $R=0.05$ and for the third case with a negative stress ratio $R$ used $P_{\max }=35 \mathrm{kN}$ and nominal $R=-1$. In all test cases, the four point bending was simulated at first to introduce a pre-existing residual plastic strain into the SENT specimen model. The results of the analysis for each test case are presented in the next chapter. Table 4-1 summarizes the test cases. The specimens were identified according to the designation employed by an experimental program conducted to verify the results of the numerical model. The experimental program is explained in the next section of this chapter.

Table 4-1 Test cases for numerical model of fatigue crack growth in residual stress fields

\begin{tabular}{|c|c|c|c|c|c|}
\hline \multirow[b]{2}{*}{ Test case } & \multirow[b]{2}{*}{$\begin{array}{l}\text { Specimen } \\
\text { Id. }\end{array}$} & \multirow[b]{2}{*}{$\begin{array}{l}\text { Specimen } \\
\text { Geometry }\end{array}$} & \multicolumn{2}{|c|}{ Fatigue Test } & \multirow{2}{*}{$\begin{array}{c}\text { Previous } \\
\text { Loading } \\
\text { History }\end{array}$} \\
\hline & & & $P_{\max }$ & $\begin{array}{c}\text { Nominal } \\
\boldsymbol{R}\end{array}$ & \\
\hline $\begin{array}{c}1 \\
(\text { Large stress ratio } R) \\
\end{array}$ & C11B10 & SENT & 55 & 0.7 & $\begin{array}{c}\text { Four point } \\
\text { bending }\end{array}$ \\
\hline $\begin{array}{c}2 \\
(\text { Low stress ratio } R)\end{array}$ & C5B3 & SENT & 35 & 0.05 & $\begin{array}{c}\text { Four point } \\
\text { bending }\end{array}$ \\
\hline $\begin{array}{c}3 \\
\text { (Negative stress ratio } \\
R)\end{array}$ & C10B8 & SENT & 35 & -1 & $\begin{array}{l}\text { Four point } \\
\text { bending }\end{array}$ \\
\hline
\end{tabular}




\subsection{Experimental verification of the fatigue crack growth model}

The experimental tests used rectangular specimens that were previously subjected to four point bending in order to introduce a residual stress field. The experimental tests were conducted in agreement with the dimensions, configurations and general conditions employed in the finite element analyses. For instance, experimental four point bending test conducted to a maximum applied load of $90 \mathrm{kN}$ and the dimensions of the rectangular specimen were identical to that of the finite element model $(254.0 \times 50.8 \times$ $12.7 \mathrm{~mm})$. Several rectangular specimens were prepared and tested so that a wellcontrolled and documented residual stress field was introduced as the one presented in chapter 3 (Figure 3-3).

In the case of the experimental FCG tests, an initial $0.5-\mathrm{mm}$ depth notch was introduced by electro-discharge machine with a wire of $0.1-\mathrm{mm}$ diameter into the rectangular specimens previously employed in the four point bending. The notch was introduced into the specimen's side with residual stresses in tension to transform the rectangular beam specimen into a SENT specimen. A FCG test was conducted through the well-controlled and documented residual stress field until final fracture or shear lip formation of the SENT specimen. The experimental FCG test conditions were set according to the finite element simulations: test case 1 with a large stress ratio $\left(P_{\max }=55 \mathrm{kN}\right.$ and $\left.R=0.7\right)$, test case 2 with a low stress ratio $\left(P_{\max }=35 \mathrm{kN}\right.$ and $\left.R=0.05\right)$ and test case 3 with a negative stress ratio $\left(P_{\max }=35 \mathrm{kN}\right.$ and $\left.\mathrm{R}=-1\right)$. For all cases, the FCG test was carried out in a servo-hydraulic machine with a load cell of capacity $100 \mathrm{kN}$ and a frequency of $25 \mathrm{~Hz}$ 
was employed. The crack length on the front- and back-faces of the SENT specimen was measured by two digital microscope cameras as a function of the number of cycles. It has been stated that the experimental tests were conducted at TU Delft by [68], but the design of the experimental tests was made between TU Delft and Carleton University with an active collaboration by this manuscript's author and its supervisors. All specimens employed to verify the FCG model were machined from the same material batch used for the baseline $d a / d N$ vs $\Delta K$ curves of 7050-T7451 aluminum alloy plate, so that the statistical variation in material properties due to microstructural conditions and chemical composition was minimized. 


\section{Chapter: Results and Discussion for the model of the Fatigue Crack Growth in Specimens with Residual Stress Fields}

Chapter 4 described the numerical model used to analyze the FCG in specimens containing residual stresses. All the finite element models presented in chapter 4 were developed in ABAQUSTM. This chapter presents the numerical results of the FCG model. In addition, collaboration with Delft University Technology (TU Delft), Faculty of Aerospace Engineering was established to conduct an experimental program and verify the FCG model. The experimental results are also presented in this chapter. This chapter is divided into seven sections. The first section presents results of the finite element model used to calculate the effective stress intensity factor $K$ without the crack closure effect. The second section presents the results of the finite element model used to calculate the effective stress intensity factor $K$ with the addition of the crack closure effect. The third section presents the results of the finite element model used to calculate the effective $K$ with the addition of the crack closure and plastic wake effects. In each of these three sections the results of the calculation of the effective parameters is presented, i.e. maximum and minimum stress intensity factors $K_{\max }$ and $K_{\min }$, stress intensity factor ranges $\Delta K$ and stress ratios $R$. The following sections present the calculations of the FCG rate and the fatigue life for each test case analyzed. The fourth section presents the results for a FCG with a large stress ratio (nominal $R=0.7$ ). The fifth section presents the results for a FCG with a low stress ratio (nominal $R=0.05$ ). The sixth section presents the results for a FCG with a negative stress ratio (nominal $R=-1$ ). The FCG rate and fatigue life for each test case analyzed are presented according to three numerical models: 1) the 
effective $K$ without the crack closure effect, 2) the effective $K$ with the crack closure effect and 3) the effective $K$ with the addition of the crack closure and plastic wake effects. Finally, the last section presents final remarks of the results of the FCG model developed in this manuscript to analyze specimens with residual stresses.

\subsection{Finite element model for the effective $K$ calculation without the crack closure effect}

This section presents the results of the calculation of the effective parameters $K_{\max }, K_{\min }$, $\Delta K$ and $R$ for each test case (Table 4-1). The effective parameters $K_{\max }$ and $K_{\min }$ were calculated with a displacement correlation method from the results of the SENT model without the crack closure effect (Figure 4-5). A four-point bending simulation was conducted to set a plastic strain field as an initial condition to the FCG test simulation. The actual residual stresses due to FCG were used for the calculations of the effective parameters $K_{\max }, K_{\min }$, rather than the pre-existing RSF. The effective $\Delta K$ and $R$ were employed by the FCG model presented in the previous chapter to predict the FCG rate as

a function of the fatigue crack length for each specimen in order to define its fatigue life.

\subsubsection{Effective $K_{\max }, K_{\min }, \Delta K$ and $R$ for a large stress ratio (test case 1)}

The results of the calculation for the effective stress intensity factors $K_{\max }$ and $K_{\min }$ are presented in Figure 5-1a for test case 1 with a large stress ratio (nominal $R=0.7$ ). Moreover, the effective stress intensity factor range $\Delta K$, which is the difference between 
$K_{\max }$ and $K_{\min }$, is also presented in Figure 5-1a. Figure 5-1b presents the results of the effective stress ratio $R$ which is the relation between $K_{\min }$ and $K_{\max }$. The results of the effective $K_{\max }, K_{\min }, \Delta K$ and $R$ were plotted in Figure 5-1 as a function of the fatigue crack lengths $a_{n}$.

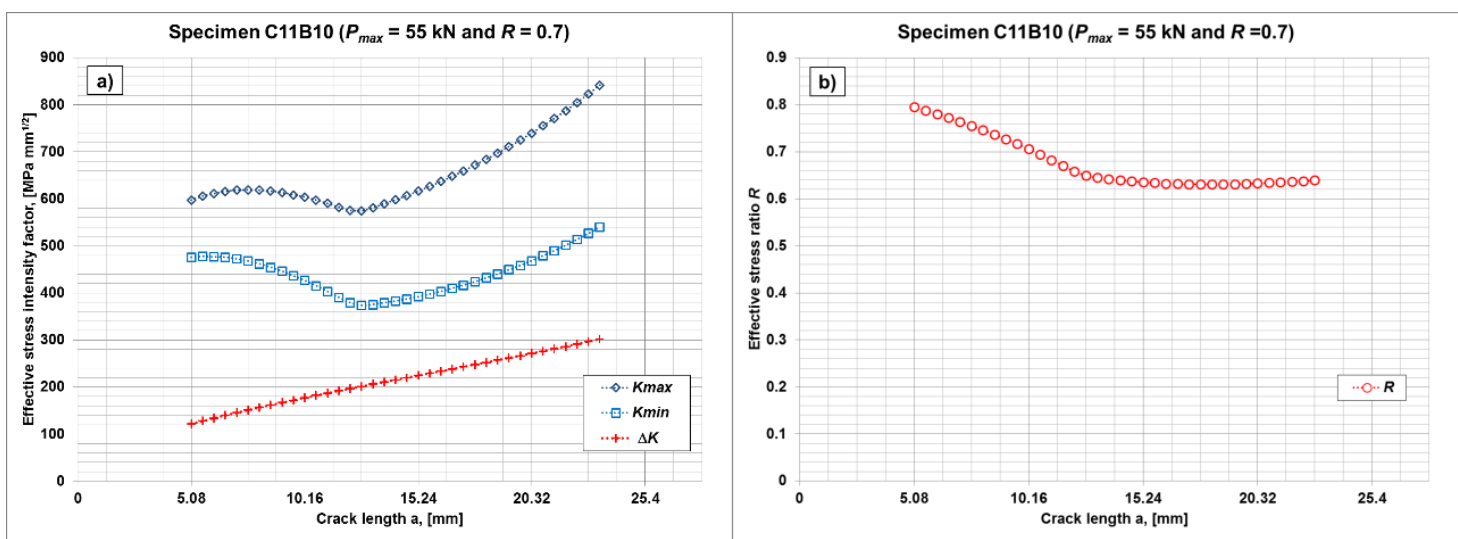

Figure 5-1 Effective stress intensity and stress ratio parameters for a large stress ratio without the crack closure effect: a) $K_{\max }, K_{\min }$ and $\Delta K$, and b) $R$.

The effective $K_{\max }$ and $K_{\min }$ presented a non-linear variation as a function of the fatigue crack lengths $a_{n}$. This non-linear variation was a result of the RSF produced by the fourpoint bending process. In the case of the effective $\Delta K$, the results indicated that the residual stresses in the SENT model subjected to the FCG test with a large stress ratio ( $R$ $=0.7)$ influenced the effective $K_{\max }$ and $K_{\min }$ in the same level. Therefore, the effective $\Delta K$ presented a linear variation over the entire range of fatigue crack lengths analyzed. Regarding the effective stress ratio $R$, a non-linear variation as a function of the fatigue crack lengths $a_{n}$ was also observed due to the presence of RSF. The data results of the effective parameters $K_{\max }, K_{\min }, \Delta K$ and $R$ are presented in Table 5-1. 
Table 5-1 Data results of the effective FCG parameters for the test case with a large stress ratio (nominal $R=0.7$ ).

\begin{tabular}{|c|c|c|c|c|c|}
\hline Id. & $\begin{array}{l}a_{n}, \\
{[m m]}\end{array}$ & $\begin{array}{l}\text { Effective } K_{\max }, \\
{\left[\text { [MPa } \text { m }^{1 / 2}\right]}\end{array}$ & $\begin{array}{l}\text { Effective } K_{\min }, \\
{\left[\mathrm{MPa} \mathrm{mm}^{1 / 2}\right]}\end{array}$ & $\begin{array}{l}\text { Effective } \Delta K, \\
\text { [MPa } \mathrm{mm}^{1 / 2} \text { ] }\end{array}$ & Effective $R$ \\
\hline 0 & 5.08 & 597.697 & 475.521 & 122.176 & 0.796 \\
\hline 1 & 5.588 & 605.990 & 477.618 & 128.373 & 0.788 \\
\hline 2 & 6.096 & 611.052 & 476.766 & 134.285 & 0.780 \\
\hline 3 & 6.604 & 616.113 & 475.915 & 140.198 & 0.772 \\
\hline 4 & \begin{tabular}{|l|l|}
7.112 \\
\end{tabular} & 618.475 & 472.611 & 145.863 & 0.764 \\
\hline 5 & 7.62 & 619.258 & 467.871 & 151.387 & 0.756 \\
\hline 6 & 8.128 & 618.587 & 461.800 & 156.787 & 0.747 \\
\hline 7 & 8.636 & 616.589 & 454.523 & 162.066 & 0.737 \\
\hline 8 & 9.144 & 613.431 & 446.185 & 167.246 & 0.727 \\
\hline 9 & 9.652 & 608.649 & 436.346 & 172.303 & 0.717 \\
\hline 10 & 10.16 & 603.867 & 426.508 & 177.359 & 0.706 \\
\hline 11 & 10.668 & 597.538 & 415.249 & 182.289 & 0.695 \\
\hline 12 & 11.176 & 590.260 & 403.104 & 187.156 & 0.683 \\
\hline 13 & 11.684 & 582.284 & 390.317 & 191.967 & 0.670 \\
\hline 14 & 12.192 & 575.686 & 378.956 & 196.731 & 0.658 \\
\hline 15 & 12.7 & 574.710 & 373.261 & 201.449 & 0.649 \\
\hline 16 & 13.208 & 581.248 & 375.111 & 206.137 & 0.645 \\
\hline 17 & 13.716 & 589.824 & 379.030 & 210.794 & 0.643 \\
\hline 18 & 14.224 & 598.144 & 382.714 & 215.430 & 0.640 \\
\hline 19 & 14.732 & 607.005 & 386.958 & 220.047 & 0.637 \\
\hline 20 & 15.24 & 616.449 & 391.793 & 224.656 & 0.636 \\
\hline 21 & 15.748 & 626.436 & 397.172 & 229.264 & 0.634 \\
\hline 22 & 16.256 & 636.967 & 403.093 & 233.874 & 0.633 \\
\hline 23 & 16.764 & 648.016 & 409.532 & 238.484 & 0.632 \\
\hline 24 & \begin{tabular}{|l|}
17.272 \\
\end{tabular} & 659.582 & 416.468 & 243.114 & 0.631 \\
\hline 25 & 17.78 & 671.676 & 423.920 & 247.756 & 0.631 \\
\hline 26 & 18.288 & 684.317 & 431.894 & 252.423 & 0.631 \\
\hline 27 & 18.796 & 697.414 & 440.291 & 257.123 & 0.631 \\
\hline 28 & 19.304 & 711.011 & 449.160 & 261.852 & 0.632 \\
\hline 29 & 19.812 & 725.151 & 458.533 & 266.618 & 0.632 \\
\hline 30 & 20.32 & 739.804 & 468.403 & 271.401 & 0.633 \\
\hline 31 & 20.828 & 755.228 & 478.923 & 276.305 & 0.634 \\
\hline 32 & 21.336 & 771.401 & 490.162 & 281.239 & 0.635 \\
\hline 33 & 21.844 & 787.574 & 501.401 & 286.173 & 0.637 \\
\hline 34 & 22.352 & 804.895 & 513.630 & 291.266 & 0.638 \\
\hline 35 & 22.86 & 822.938 & 526.511 & 296.427 & 0.640 \\
\hline 36 & 23.368 & 841.709 & 540.062 & 301.646 & 0.642 \\
\hline
\end{tabular}


The FCG analysis for this test case (nominal $R=0.7$ ) was performed up to the fatigue crack length $a_{36}=23.368 \mathrm{~mm}$. The effective parameters $K_{\max }, K_{\min }, \Delta K$ and $R$ were not calculated for fatigue crack lengths larger than $23.368 \mathrm{~mm}$, because the fracture toughness reported in the literature for the 7050-T7451 aluminum alloy $\left(K_{I c}=835 \mathrm{MPa}\right.$ $\mathrm{mm}^{1 / 2}$ ) was overpassed by the effective $K_{\max }$. The effective $K_{\max }$ was $842 \mathrm{MPa} \mathrm{mm}^{1 / 2}$ at the fatigue crack length $a_{36}=23.368 \mathrm{~mm}$.

\subsubsection{Effective $K_{\max }, K_{\min }, \Delta K$ and $R$ for a low stress ratio (test case 2)}

The results of the calculations for the effective stress intensity factors $K_{\max }$ and $K_{\min }$ are presented in Figure 5-2a for test case 2 with a low stress ratio (nominal $R=0.05$ ). In addition, the effective stress intensity factor range $\Delta K$ and the effective stress ratio $R$ are also presented in Figure 5-2a and b, respectively. The effective $K_{\max }, K_{\min }, \Delta K$ and $R$ were plotted in Figure 5-2 as a function of the fatigue crack lengths $a_{n}$.
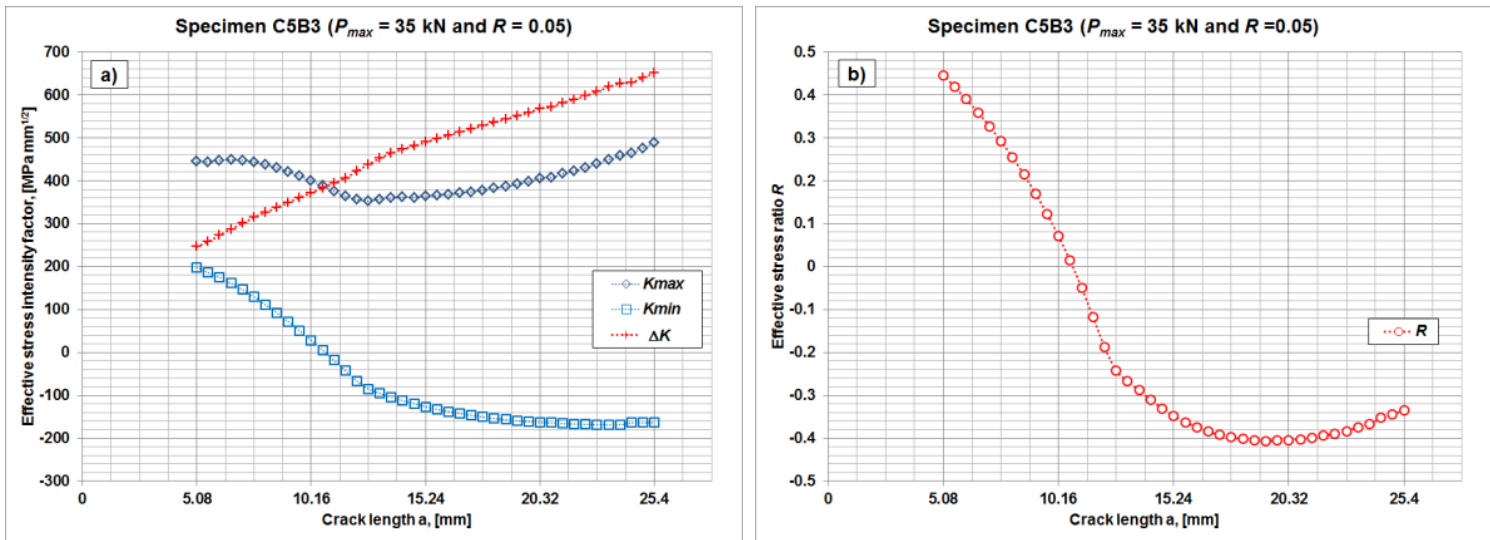

Figure 5-2 Effective stress intensity and stress ratio parameters for a low stress ratio without the crack closure effect: a) $K_{\max }, K_{\min }$ and $\Delta K$, and b) $R$. 
The SENT model with a maximum applied load $P_{\max }=35 \mathrm{kN}$ produced an effective $K_{\max }$ of approximately $445 \mathrm{MPa} \mathrm{mm}{ }^{1 / 2}$ for the initial range of fatigue crack lengths from $a_{0}=$ $5.08 \mathrm{~mm}$ to $a_{5}=7.62 \mathrm{~mm}$. This value of the effective $K_{\max }$ decreased up to $353 \mathrm{MPa}$ $\mathrm{mm}^{1 / 2}$ over the fatigue crack lengths from $a_{5}=7.62 \mathrm{~mm}$ to $a_{15}=12.7 \mathrm{~mm}$, after which it increased again up to a value of $488 \mathrm{MPa} \mathrm{mm}{ }^{1 / 2}$ over the remaining fatigue crack lengths, in the range of $a_{15}=12.7 \mathrm{~mm}$ to $a_{40}=25.4 \mathrm{~mm}$. In the case of the effective $K_{\min }$, a value of $198 \mathrm{MPa} \mathrm{mm}{ }^{1 / 2}$ was calculated for the initial fatigue crack length $a_{0}=5.08 \mathrm{~mm}$. This value of the effective $K_{\min }$ decreased down to $-169 \mathrm{MPa} \mathrm{mm}{ }^{1 / 2}$ over the fatigue crack lengths from $a_{0}=5.08 \mathrm{~mm}$ to $a_{36}=23.368 \mathrm{~mm}$, after which it increased again up to -163 $\mathrm{MPa} \mathrm{mm}{ }^{1 / 2}$ at final fatigue crack length $a_{40}=25.4 \mathrm{~mm}$. For the effective stress intensity factor range $\Delta K$, a value of $246 \mathrm{MPa} \mathrm{mm}{ }^{1 / 2}$ was calculated for the fatigue crack length $a_{0}$ $=5.08 \mathrm{~mm}$. This value of the effective $\Delta K$ increased up to $651 \mathrm{MPa} \mathrm{mm}{ }^{1 / 2}$ over the fatigue crack lengths from $a_{0}=5.08 \mathrm{~mm}$ to $a_{40}=25.4 \mathrm{~mm}$. In the case of the effective stress ratio $R$, a value of 0.45 was calculated for the initial fatigue crack length $a_{0}=5.08$ $\mathrm{mm}$. For the range of fatigue crack lengths from $a_{0}=5.08 \mathrm{~mm}$ to $a_{28}=19.304 \mathrm{~mm}$, the value of the effective $R$ decreased from 0.45 to -0.41 ; afterwards, it increased to -0.33 over the fatigue crack lengths from $a_{28}=19.304 \mathrm{~mm}$ to $a_{40}=25.4 \mathrm{~mm}$. Contrary to the previous test case with a large stress ratio, the influence of the RSF was not the same over the effective $K_{\max }$ and $K_{\min }$. As a consequence, the results of the effective $\Delta K$ were not a linear function of the fatigue crack lengths analyzed. In the case of the effective stress ratio $R$, the results indicated that the effective $R$ was also affected by the RSF as in the previous test case with a large stress ratio. However, negative values of the effective $R$ 
were calculated for the range of fatigue crack lengths from $a_{12}=11.176$ to $a_{40}=25.4 \mathrm{~mm}$ because of the low stress ratio condition.

\subsubsection{Effective $K_{\max }, K_{\min }, \Delta K$ and $R$ for a negative stress ratio (test case 3)}

Figure 5-3a presents the results for the effective stress intensity factors $K_{\max }$ and $K_{\min }$ calculated from the corresponding SENT model with a negative stress ratio and the displacement correlation method. In addition, the results for the effective stress intensity factor range $\Delta K$ is also presented in Figure 5-3a. The results of the effective stress ratio $R$ are presented in Figure 5-3b.
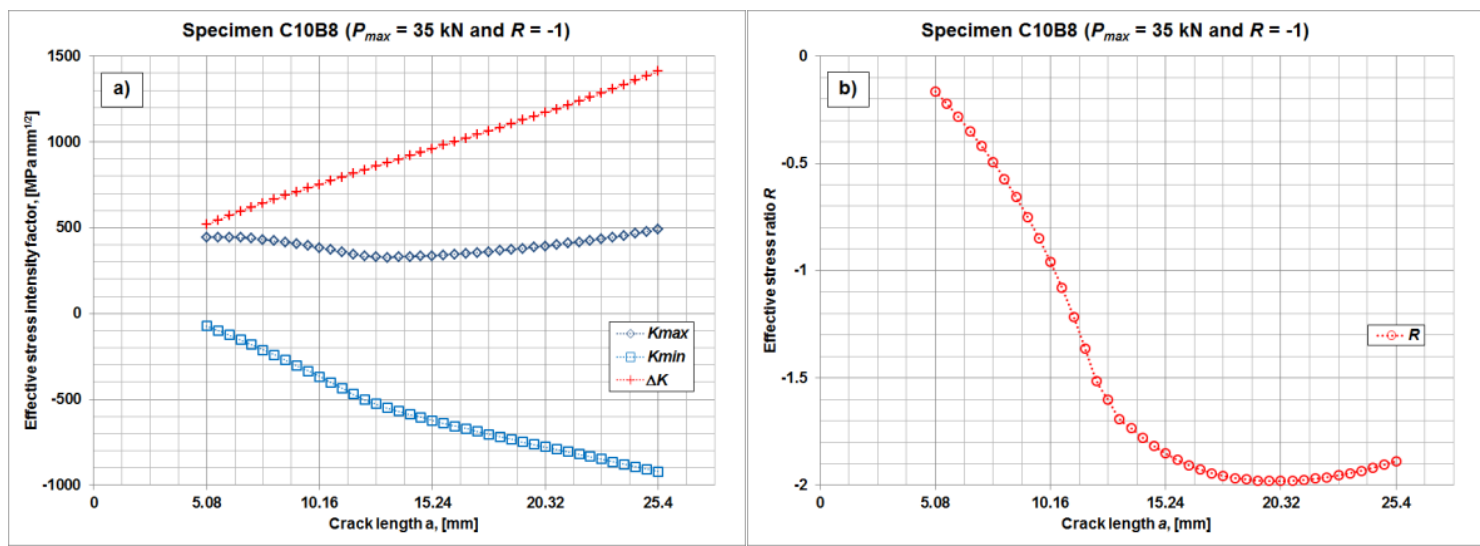

Figure 5-3 Effective stress intensity and stress ratio parameters for a negative stress ratio without the crack closure effect: a) $K_{\max }, K_{\min }$ and $\Delta K$, and b) $R$.

The initial fatigue crack length $a_{0}=5.08 \mathrm{~mm}$ exhibited a value of 445 and -73

$\mathrm{MPa} \cdot \mathrm{mm}^{1 / 2}$ for the effective $K_{\max }$ and $K_{\min }$, respectively. This and all the values of the effective FCG parameters for this test case $(R=-1)$ are reported in Table 5-2. 
Table 5-2 Data results of the effective FCG parameters for the test case with a negative stress ratio $(R=-1)$.

\begin{tabular}{|c|c|c|c|c|c|}
\hline Id. & $\begin{array}{l}a_{n}, \\
{[m m]}\end{array}$ & $\begin{array}{l}\text { Effective } K_{\max } \\
{\left[\mathrm{MPa} \mathrm{mm}^{1 / 2}\right]}\end{array}$ & $\begin{array}{l}\text { Effective } K_{\text {min }} \\
{\left[\mathrm{MPa} \mathrm{mm}^{1 / 2}\right]}\end{array}$ & 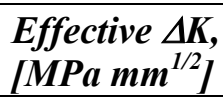 & Effective $R$ \\
\hline 0 & 5.08 & 444.632 & -73.438 & 518.070 & -0.165 \\
\hline 1 & 5.588 & 445.131 & -99.224 & 544.355 & -0.223 \\
\hline 2 & 6.096 & 443.724 & -126.163 & 569.887 & -0.284 \\
\hline 3 & 6.604 & 440.549 & -153.948 & 594.497 & -0.349 \\
\hline 4 & 7.112 & 435.986 & -182.536 & 618.521 & -0.419 \\
\hline 5 & 7.62 & 430.076 & -211.865 & 641.940 & -0.493 \\
\hline 6 & 8.128 & 422.906 & -241.921 & 664.827 & -0.572 \\
\hline 7 & 8.636 & 414.618 & -272.609 & 687.227 & -0.657 \\
\hline 8 & 9.144 & 405.290 & -303.905 & 709.195 & -0.750 \\
\hline 9 & 9.652 & 394.930 & -335.936 & 730.866 & -0.851 \\
\hline 10 & 10.16 & 383.583 & -368.507 & 752.090 & -0.961 \\
\hline 11 & 10.668 & 371.528 & -401.538 & 773.065 & -1.081 \\
\hline 12 & 11.176 & 358.627 & -435.615 & 794.242 & -1.215 \\
\hline 13 & 11.684 & 344.989 & -470.224 & 815.213 & -1.363 \\
\hline 14 & 12.192 & 332.449 & -503.465 & 835.914 & -1.514 \\
\hline 15 & 12.7 & 329.200 & -527.441 & 856.640 & -1.602 \\
\hline 16 & 13.208 & 325.950 & -551.416 & 877.367 & -1.692 \\
\hline 17 & 13.716 & 328.354 & -569.435 & 897.788 & -1.734 \\
\hline 18 & 14.224 & 330.434 & -587.791 & 918.225 & -1.779 \\
\hline 19 & 14.732 & 333.028 & -605.483 & 938.511 & -1.818 \\
\hline 20 & 15.24 & 336.137 & -622.675 & 958.812 & -1.852 \\
\hline 21 & 15.748 & 339.709 & -639.384 & 979.093 & -1.882 \\
\hline 22 & 16.256 & 343.856 & -655.696 & 999.552 & -1.907 \\
\hline 23 & 16.764 & 348.500 & -671.631 & 1020.131 & -1.927 \\
\hline 24 & 17.272 & 353.598 & -687.269 & 1040.867 & -1.944 \\
\hline 25 & 17.78 & 359.108 & -702.653 & 1061.762 & -1.957 \\
\hline 26 & 18.288 & 365.021 & -717.822 & 1082.843 & -1.967 \\
\hline 27 & 18.796 & 371.469 & -732.716 & 1104.185 & -1.972 \\
\hline 28 & 19.304 & 377.917 & -747.610 & 1125.528 & -1.978 \\
\hline 29 & 19.812 & 384.892 & -762.270 & 1147.162 & -1.980 \\
\hline 30 & 20.32 & 392.134 & -776.735 & 1168.869 & -1.981 \\
\hline 31 & 20.828 & 399.854 & -791.313 & 1191.167 & -1.979 \\
\hline 32 & 21.336 & 407.938 & -805.760 & 1213.697 & -1.975 \\
\hline 33 & 21.844 & 416.353 & -820.116 & 1236.469 & -1.970 \\
\hline 34 & 22.352 & 425.278 & -834.608 & 1259.886 & -1.963 \\
\hline 35 & 22.86 & 434.540 & -848.995 & 1283.534 & -1.954 \\
\hline 36 & 23.368 & 444.206 & -863.367 & 1307.574 & -1.944 \\
\hline 37 & 23.876 & 454.367 & -877.953 & 1332.320 & -1.932 \\
\hline 38 & 24.384 & 465.093 & -892.451 & 1357.545 & -1.919 \\
\hline 39 & 24.892 & 476.317 & -907.007 & 1383.324 & -1.904 \\
\hline 40 & 25.4 & 487.997 & -921.546 & 1409.542 & -1.888 \\
\hline
\end{tabular}


The effective stress intensity factor range $\Delta K$ presented a value of $518 \mathrm{MPa} \mathrm{mm}^{1 / 2}$ for the initial fatigue crack length $a_{0}=5.08 \mathrm{~mm}$. This value of the effective $\Delta K$ increased as a linear function of the fatigue crack length up to $1410 \mathrm{MPa} \mathrm{mm}{ }^{1 / 2}$ over the fatigue crack lengths from $a_{0}=5.08 \mathrm{~mm}$ to $a_{40}=25.4 \mathrm{~mm}$. This behavior of effective $\Delta K$ indicated that the effective $K_{\max }$ and $K_{\min }$ were equally influenced by the residual stresses in the SENT model without the crack closure effect. In the case of the effective stress ratio $R$, tensile RSF during the FCG $\left(a_{n} \leq 10.16 \mathrm{~mm}\right.$; Figure 4-14) produced larger values of the effective $R$ than the prescribed value of $R=-1$. Meanwhile, compressive RSF during the FCG $\left(a_{n}>\right.$ $10.16 \mathrm{~mm}$; Figure 4-14) produced smaller values of the effective $R$ with respect to $R=-1$. The value of the effective stress ratio $R$ decreased from -0.17 down to -1.98 over the fatigue crack lengths from $a_{0}=5.08 \mathrm{~mm}$ to $a_{30}=20.32 \mathrm{~mm}$; afterwards, the value of the effective stress ratio $R$ increased up to -1.88 over the fatigue crack lengths from $a_{30}=$ $20.32 \mathrm{~mm}$ to $a_{40}=25.4 \mathrm{~mm}$.

Similar to the previous test cases, the SENT model subjected to a FCG test with a negative stress ratio $(R=-1)$ presented a non-linear variation of the effective $K_{\max }$ and $K_{\min }$. This non-linear variation was produced by the initial plastic strain introduced during the four-point bending simulation. The behavior of the effective $K_{\max }$ seemed to indicate that the plastic zone developed during the FCG may decrease under the presence of compressive RSF. The plastic zone developed during the FCG is analyzed in the last section of this chapter. Negative values were calculated for the effective $K_{\min }$ due to the minimum applied load $P_{\min }=-35 \mathrm{kN}$ and the absence of the crack closure effect in the SENT model. These negative values of the effective $K_{\min }$ meant that the effective $R$ was also negative for all the crack lengths analyzed. 


\subsection{Finite element model for the effective $K$ calculation with the crack closure effect}

As in the previous section, the results of the effective parameters $K_{\max }, K_{\min }, \Delta K$ and $R$ from the displacement correlation method are presented for each test case (Table 4-1). However, the crack closure effect was included into the SENT model (Figure 4-15) so that negative values for the effective $K$ were not allowed. The SENT model with the crack closure effect used four-point bending simulation to set a plastic strain field as an initial condition to the FCG test simulation. Also, the actual residual stresses due to FCG were used for the calculations of the effective parameters $K_{\max }, K_{\min }$, rather than the preexisting RSF. The effective $\Delta K$ and $R$ were employed by the FCG model presented in the previous chapter to predict the FCG rate as a function of the fatigue crack length for each specimen in order to define its fatigue life.

\subsubsection{Effective $K_{\max }, K_{\min }, \Delta K$ and $R$ for a large stress ratio (test case 1)}

The results of the calculation for the effective stress intensity factors $K_{\max }$ and $K_{\min }$ produced by the SENT model with the addition of the crack closure effect were the same as those produced by the SENT model without the crack closure for nominal $R=0.7$. Therefore, the effective parameters $K_{\max }, K_{\min }, \Delta K$ and $R$ were the same as those presented in section 5.1.1 (Figure 5-1). The reason was the high stress ratio (nominal $R=$ $0.7)$ used in this test case 1 . The high magnitude of the minimum applied load $\left(P_{\min }=\right.$ $38.5 \mathrm{kN}$ ) produced conditions in which the fatigue crack was never closed during the 
FCG test simulation. The crack closure effect was not present in the SENT model with a nominal stress ratio $R=0.7$, producing an opening of the crack during the entire cyclic load range.

\subsubsection{Effective $K_{\max }, K_{\min }, \Delta K$ and $R$ for a low stress ratio (test case 2)}

Figure 5-4a presents the results for the effective stress intensity factors parameters $K_{\max }$, $K_{\min }$ calculated from the corresponding SENT model with the crack closure effect and for a low stress ratio (nominal $R=0.05$ ). In addition, the results for the effective stress intensity factor $\Delta K$ are also presented in Figure 5-4a. The results of the effective stress ratio $R$ are presented in Figure 5-4b. The effective $K_{\max }, K_{\min }, \Delta K$ and $R$ were plotted as a function of the crack length $a_{n}$.
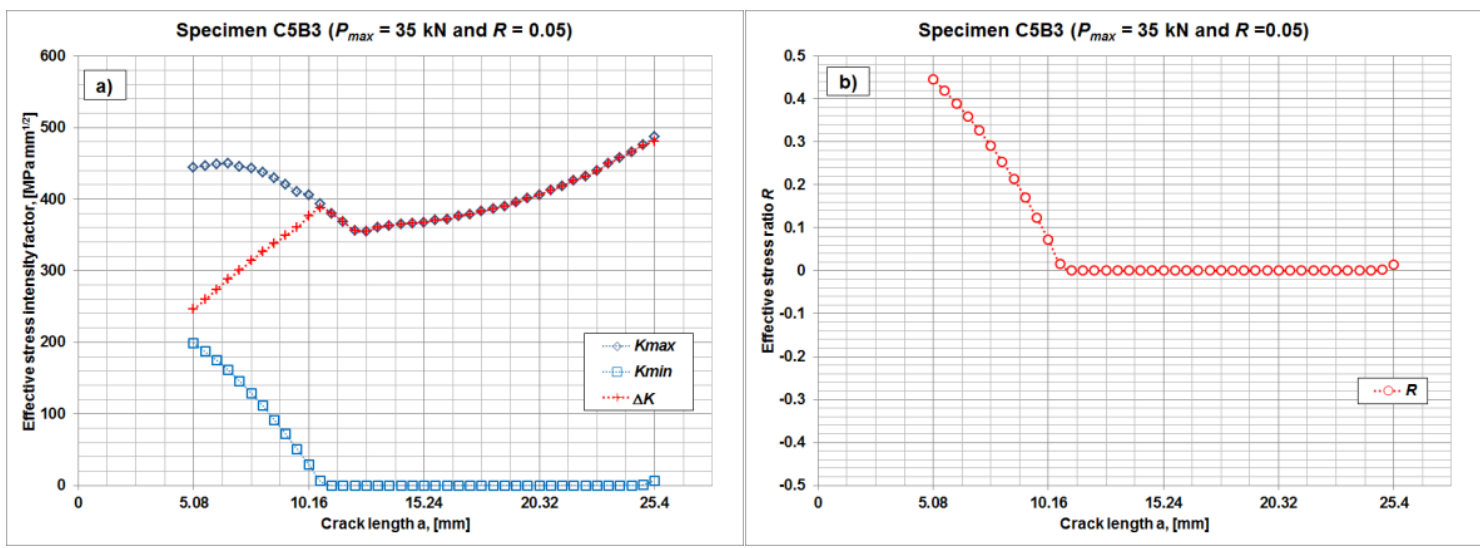

Figure 5-4 Effective stress intensity and stress ratio parameters for a low stress ratio with the crack closure effect: a) $K_{\max }, K_{\min }$ and $\Delta K$, and b) $R$. 
The SENT model with a maximum applied load $\left(P_{\max }=35 \mathrm{kN}\right)$ produced an effective $K_{\max }$ of approximately $445 \mathrm{MPa} \mathrm{mm}{ }^{1 / 2}$ over the fatigue crack lengths from $a_{0}=5.08 \mathrm{~mm}$ to $a_{5}=7.62 \mathrm{~mm}$. Afterwards, the effective $K_{\max }$ decreased down to $355 \mathrm{MPa} \mathrm{mm}{ }^{1 / 2}$ over the fatigue crack lengths from $a_{5}=7.62 \mathrm{~mm}$ to $a_{15}=12.7 \mathrm{~mm}$. Finally, the effective $K_{\max }$ increased up to $488 \mathrm{MPa} \mathrm{mm}{ }^{1 / 2}$ over the fatigue crack lengths from $a_{15}=12.7 \mathrm{~mm}$ to $a_{40}=$ $25.4 \mathrm{~mm}$. These results of the effective $K_{\max }$ for the SENT model with the crack closure effect and for a low stress ratio (nominal $R=0.05$ ) were identical with respect to the corresponding ones without the crack closure effect (Figure 5-2). The fatigue crack was completely open in the SENT model with the maximum applied load during the FCG test simulation. The result of the effective $K_{\min }$ was $198 \mathrm{MPa} \mathrm{mm}{ }^{1 / 2}$ for the initial fatigue crack length $a_{0}=5.08 \mathrm{~mm}$. This value of the effective $K_{\min }$ decreased down to zero over the fatigue crack lengths from $a_{0}=5.08 \mathrm{~mm}$ to $a_{12}=11.176 \mathrm{~mm}$. The value of the effective $K_{\min }$ was zero for the fatigue crack lengths from $a_{12}=11.176 \mathrm{~mm}$ to $a_{38}=24.384$ $\mathrm{mm}$, and the effective $K_{\min }$ increased again up to $7 \mathrm{MPa} \mathrm{mm}^{1 / 2}$ over the fatigue crack lengths from $a_{38}=24.384 \mathrm{~mm}$ to $a_{40}=25.4 \mathrm{~mm}$. The result of the effective stress intensity factor range $\Delta K$ was $246 \mathrm{MPa} \mathrm{mm}{ }^{1 / 2}$ for the initial fatigue crack length analyzed $\left(a_{0}=5.08 \mathrm{~mm}\right)$. The value of the effective $\Delta K$ increased as a linear function of the crack length up to $387 \mathrm{MPa} \mathrm{mm}{ }^{1 / 2}$ over the fatigue crack lengths from $a_{0}=5.08 \mathrm{~mm}$ to $a_{12}=$ $11.176 \mathrm{~mm}$. After that, the value of the effective $\Delta K$ was a non-linear function of the crack length for the remaining fatigue crack lengths. In addition, the value of the effective $\Delta K$ overlapped the results of the effective $K_{\max }$ for the fatigue crack lengths from $a_{12}=$ $11.176 \mathrm{~mm}$ to $a_{38}=24.384 \mathrm{~mm}$. The non-linear behavior for the results of the effective $\Delta K$ was produced when the effective $K_{\min }$ was equal to zero, which implied that the 
fatigue crack in the SENT model was closed for the minimum applied load $\left(P_{\min }=1.75\right.$ $\mathrm{kN})$. This indicated a crack closure effect in the SENT model with a low stress ratio for the fatigue crack lengths from $a_{12}=11.176 \mathrm{~mm}$ to $a_{38}=24.384 \mathrm{~mm}$. The value of the effective stress ratio $R$ was 0.45 for the initial fatigue crack length $a_{0}=5.08 \mathrm{~mm}$. The result of the effective $R$ decreased down to zero over the fatigue crack lengths from $a_{0}=$ $5.08 \mathrm{~mm}$ to $a_{12}=11.176 \mathrm{~mm}$; afterwards, the value of the effective $R$ was zero for the fatigue crack lengths from $a_{12}=11.176 \mathrm{~mm}$ to $a_{38}=24.384 \mathrm{~mm}$. Finally, the effective $R$ increased up to 0.01 over the fatigue crack lengths from $a_{38}=24.384 \mathrm{~mm}$ to $a_{40}=25.4$ $\mathrm{mm}$. The residual stresses induced a crack closure effect over a range of fatigue crack lengths $\left(a_{12}\right.$ to $\left.a_{38}\right)$. This crack closure effect caused zero values for the effective $K_{\min }$. As a result, the effective $R$ was zero for the range of fatigue crack lengths ( $a_{12}$ to $\left.a_{38}\right)$ and the effective $\Delta K$ was equal to the effective $K_{\max }$.

\subsubsection{Effective $K_{\max }, K_{\min }, \Delta K$ and $R$ for a negative stress ratio (test case 3)}

The results for the effective stress intensity factors $K_{\max }$ and $K_{\min }$ with a negative stress ratio (nominal $R=-1$ ) are presented in Figure 5-5. As a remainder, the effective $K_{\max }$ and $K_{\min }$ were calculated by a displacement correlation method that used the numerical results of the SENT model with the crack closure effect (Figure 4-15) as explained in the previous chapter. In addition, the effective stress intensity factor range $\Delta K$ and the effective stress ratio $R$ are also presented in Figure 5-5a and b, respectively. The effective $K_{\max }, K_{\min }, \Delta K$ and $R$ were plotted in Figure 5-5 as a function of the fatigue crack lengths $a_{n}$. 

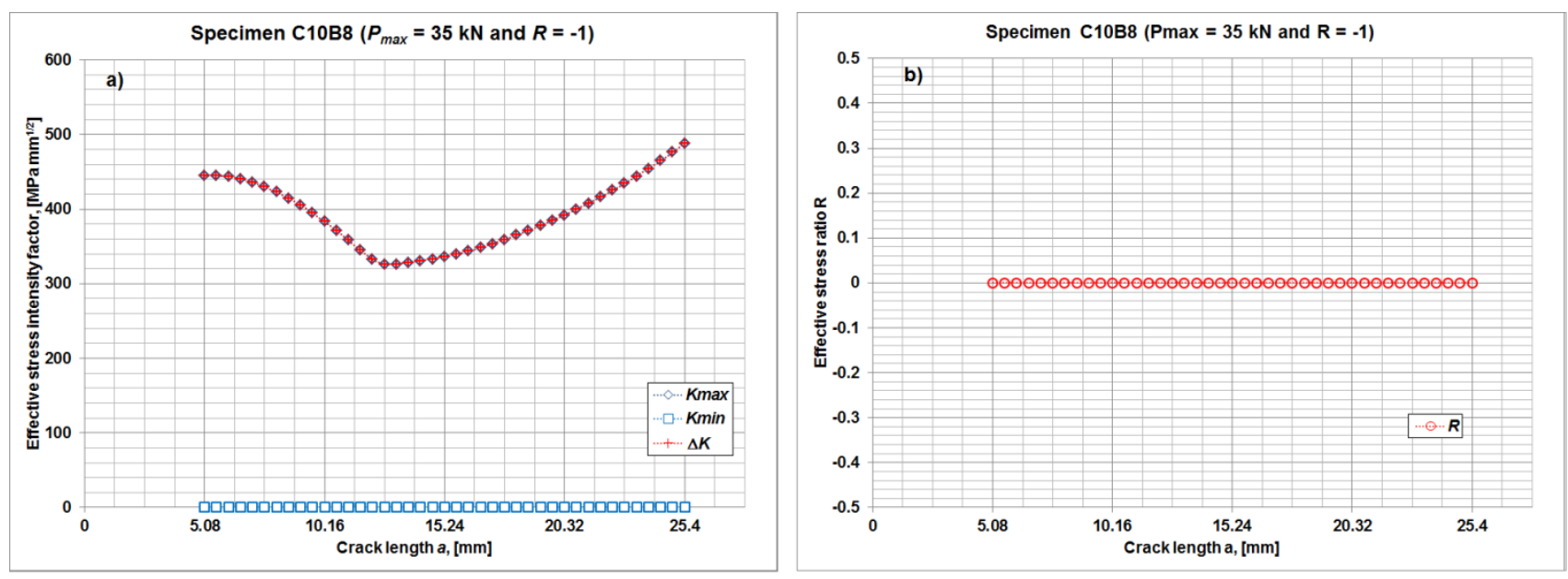

Figure 5-5 Effective stress intensity and stress ratio parameters for a negative stress ratio with the crack closure effect: a) $K_{\max }, K_{\min }$ and $\Delta K$, and b) $R$.

The minimum applied load $\left(P_{\min }=-35 \mathrm{kN}\right)$ for the SENT model with residual stresses was negative, so that the result for the effective $K_{\min }$ was zero for all the fatigue crack lengths analyzed $\left(a_{0}=5.08 \mathrm{~mm}\right.$ to $\left.a_{40}=25.4 \mathrm{~mm}\right)$. In the case of the effective $K_{\max }$, the crack closure effect was overcome by the maximum applied load $\left(P_{\max }=35 \mathrm{kN}\right)$ and the calculated values of the effective $K_{\max }$ were identical between this test case with a negative stress ratio and the previous one with a low stress ratio. The effective stress intensity factor range $\Delta K$ was a non-linear function of the crack length, which was equal to the effective $K_{\max }$. The value of the effective stress ratio $R$ was zero for all the fatigue crack lengths analyzed. 


\subsection{Finite element model for the effective $K$ calculation with the addition of the crack closure and plastic wake effects}

The SENT model with residual stresses used for the determination of the effective stress intensity factors $K_{\max }$ and $K_{\min }$ with the crack closure effect (Figure 4-15) was modified to include the plastic wake effect. A refined mesh (Figure 4-20) around the crack tip was used to capture the plastic zone during the FCG test simulation. As in the previous sections, the actual residual stresses in the SENT model due to FCG were used for the calculations of the effective parameters $K_{\max }, K_{\min }$, rather than the pre-existing RSF. This section presents the results from the displacement correlation method of the effective stress intensity factor $K_{\max }$ and $K_{\min }$ with the addition of the crack closure and plastic wake effects for three test cases: 1) with a large stress ratio, 2) with a low stress ratio and 3) with a negative stress ratio. In addition, the effective parameters $\Delta K$ and $R$ are presented for each test case. The effective $\Delta K$ and $R$ were employed by the FCG model presented in the previous chapter to calculate the FCG rate as a function of the fatigue crack length for each test case in order to define its fatigue life.

\subsubsection{Effective $K_{\max }, K_{\min }, \Delta K$ and $R$ for a large stress ratio (test case 1)}

Figure 5-6a presents the results for the effective stress intensity factors $K_{\max }$ and $K_{\min }$ calculated from the corresponding SENT model with the addition of the crack closure and plastic wake effects for a large stress ratio (nominal $R=0.7$ ). In addition, the results for the effective stress intensity factor range $\Delta K$ are also presented in Figure 5-6a. The 
results of the effective stress ratio $R$ are presented in Figure 5-6b. The effective parameters $K_{\max }, K_{\min }, \Delta K$ and $R$ were plotted as a function of the crack length $a_{n}$.
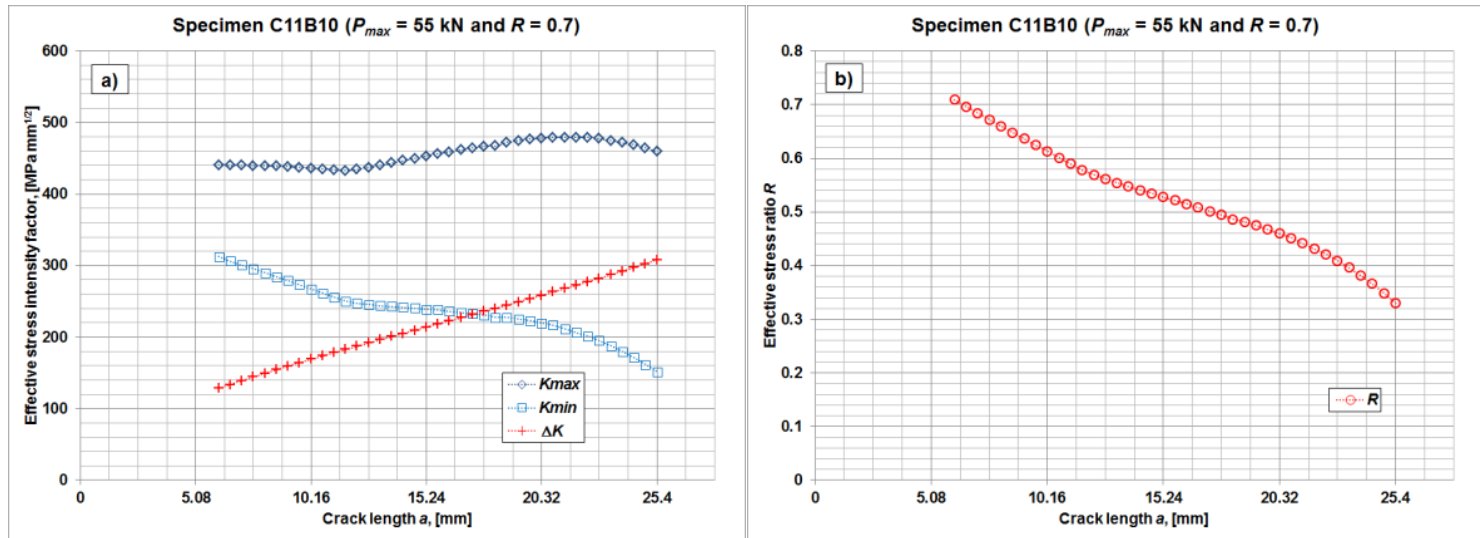

Figure 5-6 Effective stress intensity and stress ratio parameters for a large stress ratio with the addition of the crack closure and plastic wake effects: a) $K_{\max }, K_{\min }$ and $\Delta K$, and b) $R$.

For the test case with a large stress ratio (nominal $R=0.7$ ), the SENT model with the addition of the crack closure and plastic wake effects (Figure 5-6) produced smaller values of the effective $K_{\max }, K_{\min }$ and $R$ with respect to the SENT model without the crack closure effect (Figure 5-1). Regarding the effective stress intensity factor range $\Delta K$, the value for the initial fatigue crack length $a_{0}=5.08 \mathrm{~mm}$ was $128 \mathrm{MPa} \mathrm{mm}^{1 / 2}$. This value of the effective $\Delta K$ increased as a linear function of the crack length up to $307 \mathrm{MPa} \mathrm{mm}{ }^{1 / 2}$ over the fatigue crack lengths from $a_{0}=5.08 \mathrm{~mm}$ to $a_{40}=25.4 \mathrm{~mm}$. The values of the effective $\Delta K$ were very similar between the SENT model with the addition of the crack closure and plastic wake effects (Figure 5-6), and the corresponding model without the crack closure effect (Figure 5-1). In relation to the effective stress ratio $R$, the calculated 
value for the initial fatigue crack length $a_{0}=5.08 \mathrm{~mm}$ was 0.7 . This value of the effective $R$ decreased up to 0.32 over the fatigue crack lengths from $a_{0}=5.08 \mathrm{~mm}$ to $a_{40}=25.4$ mm.

\subsubsection{Effective $K_{\max }, K_{\min }, \Delta K$ and $R$ for a low stress ratio (test case 2)}

The results of the calculation for the effective stress intensity factors $K_{\max }$ and $K_{\min }$ are presented in Figure 5-7a for a FCG with a low stress ratio (nominal $R=0.05$ ). In addition, the effective stress intensity factor range $\Delta K$ is presented in Figure 5-7a. The results of the calculation for the effective stress ratio $R$ are presented in Figure 5-7b. These results of the effective $K_{\max }, K_{\min }, \Delta K$ and $R$ were produced by the SENT model with the addition of the crack closure and plastic wake effects.
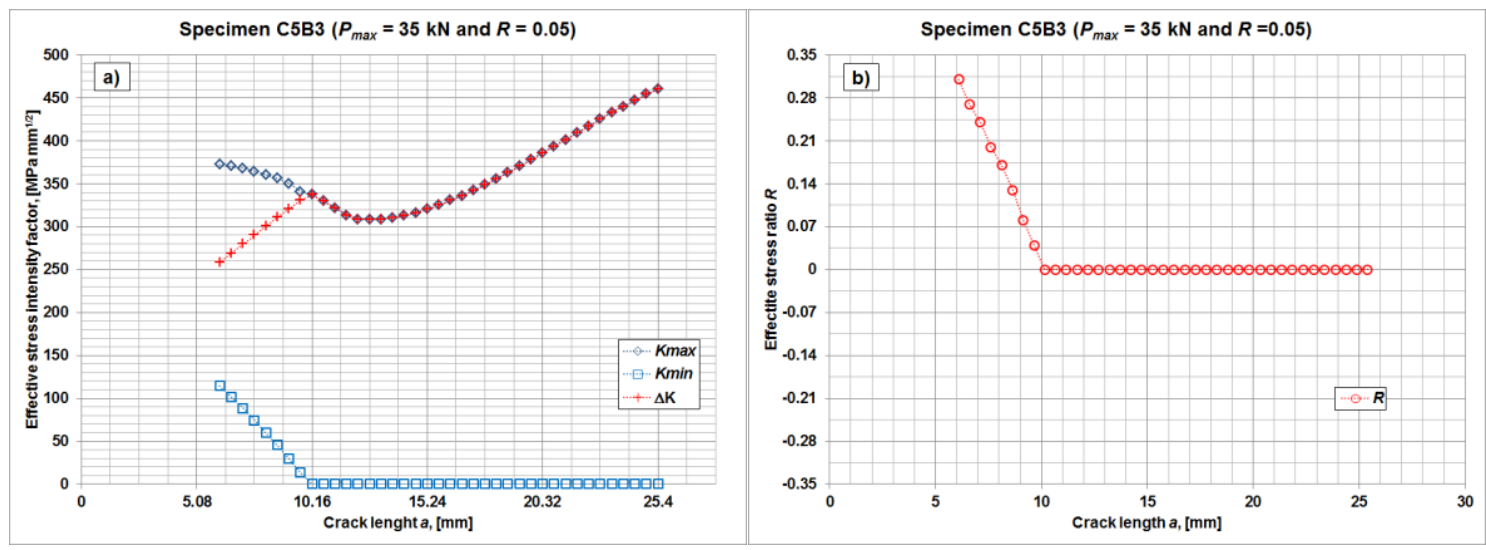

Figure 5-7 Effective stress intensity and stress ratio parameters for a low stress ratio with the addition of the crack closure and plastic wake effects: a) $K_{\max }, K_{\min }$ and $\Delta K$, and b) $R$. 
As previously mentioned, the crack closure effect in the SENT model with residual stresses subjected to a low stress ratio caused zero values of the effective $K_{\min }$ over the fatigue crack lengths from $a_{12}=11.176 \mathrm{~mm}$ to $a_{38}=24.384 \mathrm{~mm}$ (Figure 5-4). However, the incorporation of the plastic wake effect produced zero values of the effective $K_{\min }$ over the fatigue crack lengths from $a_{10}=10.16 \mathrm{~mm}$ to $a_{40}=25.4 \mathrm{~mm}$ (Figure 5-7). Therefore, the effective stress ratio $R$ exhibited a zero value over the same range of fatigue crack lengths ( $a_{10}=10.16 \mathrm{~mm}$ to $\left.a_{40}=25.4 \mathrm{~mm}\right)$. In addition, the incorporation of the plastic wake effect produced smaller values for the effective $K_{\max }, K_{\min }, \Delta K$ and $R$ (Figure 5-7) than the values produced by the SENT model with the crack closure effect (Figure 5-4). The data results of the effective parameters $K_{\max }, K_{\min }, \Delta K$ and $R$ from the SENT model with the addition of the crack closure and plastic wake effect are presented in Table 5-3.

The fatigue crack was completely open during the FCG from the initial crack length up to the crack length $a_{10}=10.16 \mathrm{~mm}$ as shown by the positive values of the effective $K_{\min }$ for this range of crack lengths. A linear variation of the effective $\Delta K$ was observed for the range of fatigue crack lengths from $a_{2}=6.096 \mathrm{~mm}$ to $a_{10}=10.16 \mathrm{~mm}$; thus, the effective $K_{\max }$ and $K_{\min }$ were equally influenced by the presence of a tensile RSF and the crack was completely open during the FCG. Meanwhile, compressive RSF produced a crack closure effect for the range of fatigue crack lengths from $a_{10}=10.16 \mathrm{~mm}$ to $a_{40}=25.4 \mathrm{~mm}$ and the effective $\Delta K$ was equal to the effective $K_{\max }$. 
Table 5-3 Data results of the effective FCG parameters for the test case with a low stress ratio (nominal $R=0.05$ ).

\begin{tabular}{|c|c|c|c|c|c|}
\hline Id. & $\begin{array}{l}a_{n}, \\
{[m m]}\end{array}$ & 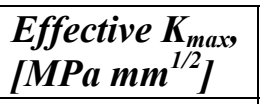 & \begin{tabular}{|l|} 
Effective $\boldsymbol{K}_{\min }$ \\
{$\left[{\left.\text { [MPa } \mathrm{m}^{1 / 2}\right]}\right]$} \\
\end{tabular} & $\begin{array}{l}\text { Effective } \Delta K, \\
{\left[M P a m^{1 / 2}\right]}\end{array}$ & Effective $R$ \\
\hline 0 & - & - & - & - & - \\
\hline 1 & - & - & - & - & - \\
\hline 2 & 6.096 & 372.64 & 114.7 & 257.96 & 0.31 \\
\hline 3 & 6.604 & 370.72 & 101.7 & 269.04 & 0.27 \\
\hline 4 & 7.112 & 368.26 & 88.38 & 279.89 & 0.24 \\
\hline 5 & 7.62 & 364.56 & 74.12 & 290.43 & 0.2 \\
\hline 6 & 8.128 & 360.65 & 59.86 & 300.79 & 0.17 \\
\hline 7 & 8.636 & 356.21 & 45.32 & 310.89 & 0.13 \\
\hline 8 & 9.144 & 350.33 & 29.49 & 320.84 & 0.08 \\
\hline 9 & 9.652 & 340.55 & 13.58 & 330.96 & 0.04 \\
\hline 10 & 10.16 & 337.36 & 0 & 337.36 & 0 \\
\hline 11 & 10.668 & 329.96 & 0 & 329.96 & 0 \\
\hline 12 & 11.176 & 321.79 & 0 & 321.79 & 0 \\
\hline 13 & 11.684 & 313.47 & 0 & 313.47 & 0 \\
\hline 14 & 12.192 & 308.83 & 0 & 308.83 & 0 \\
\hline 15 & 12.7 & 308.12 & 0 & 308.12 & 0 \\
\hline 16 & 13.208 & 308.45 & 0 & 308.45 & 0 \\
\hline 17 & 13.716 & 310.13 & 0 & 310.13 & 0 \\
\hline 18 & 14.224 & 312.79 & 0 & 312.79 & 0 \\
\hline 19 & 14.732 & 316.25 & 0 & 316.25 & 0 \\
\hline 20 & 15.24 & 320.48 & 0 & 320.48 & 0 \\
\hline 21 & 15.748 & 325.31 & 0 & 325.31 & 0 \\
\hline 22 & 16.256 & 330.7 & 0 & 330.7 & 0 \\
\hline 23 & 16.764 & 336.23 & 0 & 336.23 & 0 \\
\hline 24 & 17.272 & 342.31 & 0 & 342.31 & 0 \\
\hline 25 & 17.78 & 348.83 & 0 & 348.83 & 0 \\
\hline 26 & 18.288 & 355.84 & 0 & 355.84 & 0 \\
\hline 27 & 18.796 & 362.99 & 0 & 362.99 & 0 \\
\hline 28 & 19.304 & 370.3 & 0 & 370.3 & 0 \\
\hline 29 & 19.812 & 378.15 & 0 & 378.15 & 0 \\
\hline 30 & 20.32 & 386 & 0 & 386 & 0 \\
\hline 31 & 20.828 & 393.62 & 0 & 393.62 & 0 \\
\hline 32 & 21.336 & 401.28 & 0 & 401.28 & 0 \\
\hline 33 & 21.844 & 409.28 & 0 & 409.28 & 0 \\
\hline 34 & 22.352 & 417.38 & 0 & 417.38 & 0 \\
\hline 35 & 22.86 & 425.08 & 0 & 425.08 & 0 \\
\hline 36 & 23.368 & 433.13 & 0 & 433.13 & 0 \\
\hline 37 & 23.876 & 440.05 & 0 & 440.05 & 0 \\
\hline 38 & 24.384 & 447.25 & 0 & 447.25 & 0 \\
\hline 39 & 24.892 & 454.43 & 0 & 454.43 & 0 \\
\hline 40 & 25.4 & 460.32 & 0 & 460.32 & 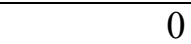 \\
\hline
\end{tabular}




\subsubsection{Effective $K_{\max }, K_{\min }, \Delta K$ and $R$ for a negative stress ratio (test case 3)}

Figure 5-8a presents the calculated values of the effective stress intensity factors $K_{\max }$ and $K_{\min }$ as a function of the crack length $a_{i}$ for the test case 3 with a negative stress ratio. In addition, Figure 5-8a presents the effective stress intensity factor range $\Delta K$. The effective $K_{\max }$ and $K_{\min }$ were produced by the SENT model with the addition of the crack closure and plastic wake effects. Figure 5-8b presents the results for the effective stress ratio $R$ as a function of the crack length.
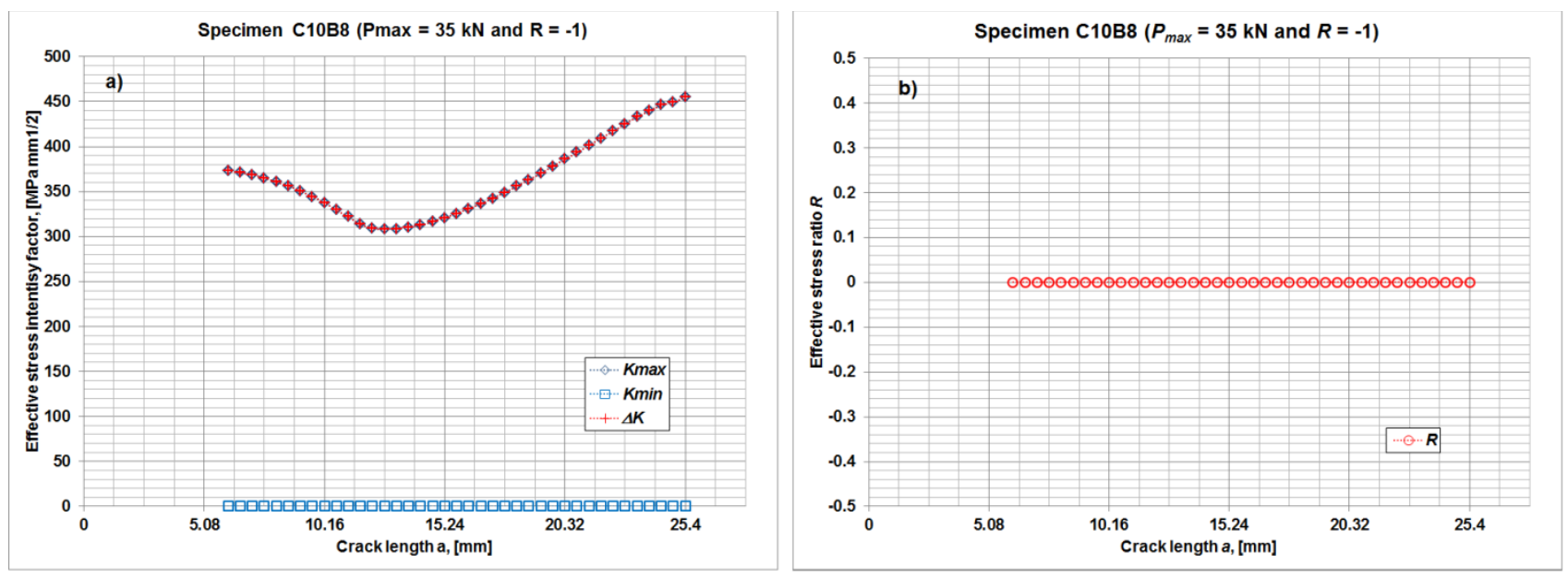

Figure 5-8 Effective stress intensity and ratio parameters for a negative stress ratio with the addition of the crack closure and plastic wake effects: a) $K_{\max }, K_{\min }$ and $\Delta K$, and b) $R$.

The calculated values for the effective stress intensity factor $K_{\max }$ were equal to the effective stress intensity factor range $\Delta K$, because the fatigue crack in the SENT model was closed at the minimum applied load $\left(P_{\min }=-35 \mathrm{kN}\right)$ and $K_{\min }$ was zero for all the fatigue crack lengths analyzed. The effective stress intensity factor range $\Delta K$ was 373 
$\mathrm{MPa} \cdot \mathrm{mm}^{1 / 2}$ for the initial fatigue crack length $a_{0}=5.08 \mathrm{~mm}$. This value of the effective $\Delta K$ decreased down to $308 \mathrm{MPa} \cdot \mathrm{mm}^{1 / 2}$ over the fatigue crack lengths from $a_{0}=5.08 \mathrm{~mm}$ to $a_{15}=12.7 \mathrm{~mm}$. Then, the value of the effective $\Delta K$ increased up to $464 \mathrm{MPa} \cdot \mathrm{mm}^{1 / 2}$ over the fatigue crack lengths from $a_{15}=12.7 \mathrm{~mm}$ to $a_{40}=25.4 \mathrm{~mm}$. The effective stress ratio $R$ was zero for all the fatigue crack lengths analyzed.

\subsection{Fatigue crack growth rate and fatigue life calculations for test case 1 (nominal $R=0.7$ )}

The conditions of the FCG analysis for test case 1 were set at a large stress ratio (nominal $R=0.7$ ) with a maximum applied cyclic load of $55 \mathrm{kN}$. These conditions corresponded to the specimen $\mathrm{C} 11 \mathrm{~B} 10$ according to the test matrix presented in Table 4-1. The specimen C11B10 was previously subjected to four-point bending to introduce a residual stress field. The calculation of the effective $K_{\max }$ and $K_{\min }$ was done with a displacement correlation method, which used the numerical results from the simulation of a FCG test of a SENT model. A commercially available-finite element program (ABAQUSTM) was used to create the SENT model and conduct the FCG simulation. Three SENT models were developed: 1) without the crack closure effect, 2) with the crack closure effect, and 3) with the addition of the crack closure and plastic wake effects.

For a large stress ratio (test case 1), the results of the calculation for the effective $\Delta K$ (Figure 5-1) produced by the SENT models with and without the crack closure effect demonstrated that the crack was completely open during the FCG. The crack closure effect was not present for the FCG in the SENT model with a large stress ratio (nominal 
$R=0.7)$. The Harter T-method was used to interpolate between the experimental baseline FCG curves of Figure 4-1 (7050-T7451 aluminum alloy), in order to calculate new baseline $d a / d N$ vs. $\Delta K$ curves for stress ratios of interest as shown in Figure 4-3. The FCG rate was determined from the baseline $d a / d N$ vs. $\Delta K$ curves for the 7050-T7451 aluminum alloy (base material) as a function of the calculated effective $\Delta K$ and $R$. The results of the FCG rate for the SENT model with a large stress ratio (test case 1) are shown in Figure 5-9 as a function of the crack length. Two plots are shown; Figure 5-9a presents the full range of crack lengths for specimen C11B10, and Figure 5-9b presents a zoom-in to Figure 5-9a for the range of crack lengths from $a_{6}=6.096 \mathrm{~mm}$ to $a_{23}=16.764$ $\mathrm{mm}$ where the initial residual stresses after the four-point bending test were in compression. In addition, Figure 5-9 also shows the experimental results of the FCG rate for the front- and back-face of the SENT specimen of 7050-T7451 aluminum alloy (data in dark-blue with plus markers and blue colors with times markers). Horizontal bars are included in the plots to specify the distribution of the initial residual stresses produced by the four-point bending in the specimen C11B10. The blue color in the bar indicates a tensile stress and the red color indicates a compressive stress. The calculations of the FCG rate were done from an initial fatigue crack length $a_{0}=5.08 \mathrm{~mm}$ up to $a_{40}=25.4$ $\mathrm{mm}$. The crack length increments $\Delta a$ were of $0.508 \mathrm{~mm}$. In general, the FCG rate calculations presented a better correlation with respect to the experimental values for fatigue crack lengths in the range of $a_{0}=5.08 \mathrm{~mm}$ to a $a_{20}=15.24 \mathrm{~mm}$ than for fatigue crack lengths in the range of $a_{20}=15.24 \mathrm{~mm}$ to $a_{40}=25.4 \mathrm{~mm}$. 


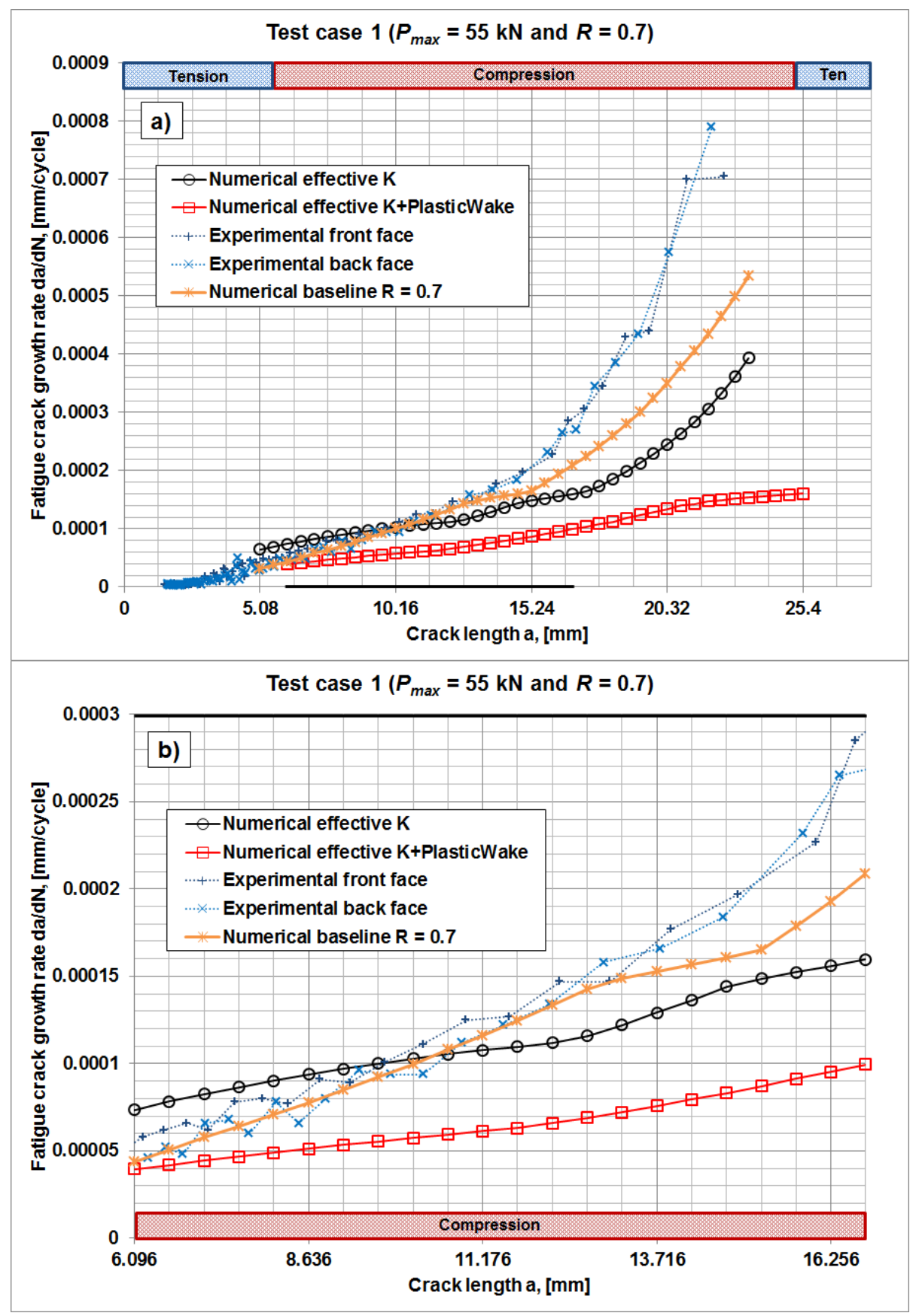

Figure 5-9 Fatigue crack growth rate as a function of the crack length for test case 1 with a large stress ratio $\left(P_{\max }=55 \mathrm{kN}\right.$; nominal $R=0.7$; C11B10 specimen): a) Full range of crack lengths, and b) range of crack lengths from $a_{6}=6.096 \mathrm{~mm}$ to $a_{23}=16.764 \mathrm{~mm}$. 
The FCG rate calculations from the SENT model without the crack closure effect (Numerical effective $K$; data in black color and open circle markers; Figure 5-9) were consistently larger than the calculations of the corresponding growth rate using the SENT model with the crack closure and plastic wake effects (Numerical effective $K+$ PlasticWake; data in red color and rectangle markers; Figure 5-9). The calculated FCG rates did not show a FCG retardation in spite of the initial residual stresses (after the fourpoint bending) in compression (Figure 5-9b); this lack of FCG retardation was verified by the experimental measurements of the FCG rate in specimen C11B10 where a decrease in the FCG rate was not observed. In the case of the numerical model with the effective $K$, the FCG rate was overestimated for fatigue crack lengths in the range of $a_{0}=5.08 \mathrm{~mm}$ to $a_{10}=10.16 \mathrm{~mm}$, while for fatigue crack lengths larger than $a_{10}=10.16 \mathrm{~mm}$ the FCG rate was underestimated. Just for the crack length of $a_{10}=10.16 \mathrm{~mm}$, the calculated FCG rate was within the range of the experimental values for the front- and back-face of the SENT specimen. The analysis was conducted until the fatigue crack length $a_{36}=23.368 \mathrm{~mm}$ and after that fatigue crack length the calculation of $d a / d N$ was not determined, because the computed $K_{\max }$ was higher than the fracture toughness of the material. The SENT model with the crack closure effect was not employed for test case 1, because the effective parameters $K_{\max }, K_{\min }$, were the same as those from the SENT model without the crack closure effect (Figure 5-1). For the case of the SENT model with the addition of the crack closure and plastic wake effects, the calculated FCG rates were lower than the corresponding growth rates from the numerical model with the effective $K$. The calculated values of the FCG rate indicated that for the large stress ratio $R=0.7$ used in specimen C11B10, the crack closure and plastic wake effects should not be considered. 
Figure 5-10 presents the crack profile during the FCG test simulation at maximum and minimum applied loads according to the finite element model for the effective $K$ without the addition of the crack closure and plastic wake effects.

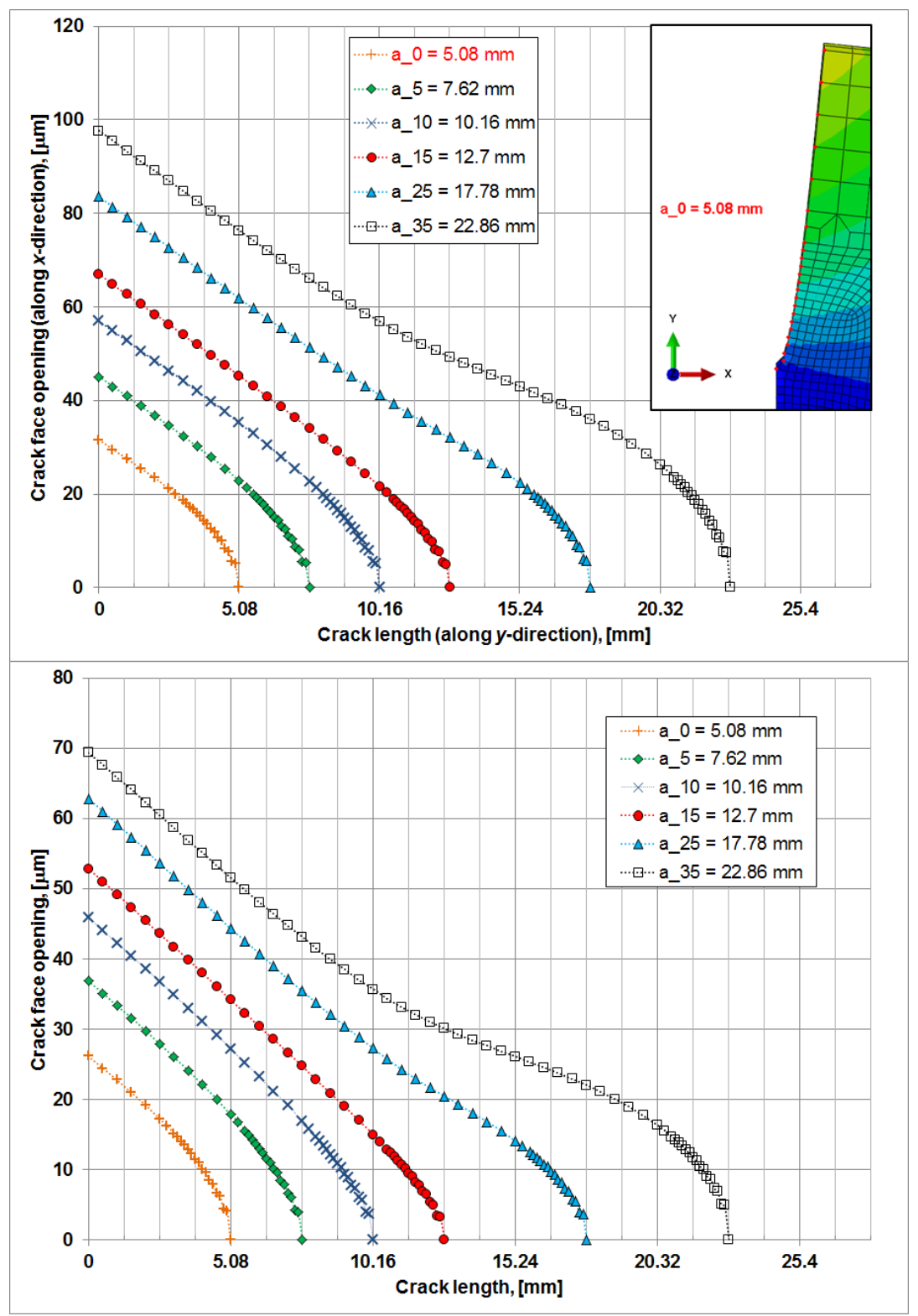

Figure 5-10 Crack profile during the fatigue crack growth test simulation for test case 1 at $\operatorname{nominal} R=0.7$ : a) maximum applied load $\left(P_{\max }=55 \mathrm{kN}\right)$ and $\left.\mathrm{b}\right)$ minimum applied load $\left(P_{\min }=38.5 \mathrm{kN}\right)$. 
The crack profile showed that the crack was open for the complete FCG simulation. It seemed that the plastic wake effect was not important for the calculation of the FCG rates because of the absence of the crack closure effect. In addition, the calculation of the FCG rate in RSF for a large applied stress ratio (nominal $R=0.7$ ) may be independent of small variations in the effective stress ratio. According to the calculations from the SENT model without the crack closure and plastic wake effects (Figure 5-1), the effective stress ratio $R$ presented values within the range of 0.8 to 0.63 . For the crack length of 10.16 $\mathrm{mm}$, which was the only crack length where the FCG rate was accurately calculated, the effective $R$ was 0.7 .

To further analyze if small variations of the effective stress ratio should not be considered by the FCG model, the FCG rate was also calculated according to the SENT model without the crack closure effect and the baseline FCG curve at nominal $R=0.7$ (Numerical baseline $R=0.7$, data in orange color and times markers; Figure 5-9) for the 7050-T7451 aluminum alloy. Figure 5-9 also presents these calculations of the FCG rate (Numerical baseline $R=0.7$ ) that did not employ the Harter T-method, but instead the effective $K$ was correlated with the baseline FCG curve of the 7050-T7451 aluminum alloy (free of four-point bending) at $R=0.7$ (Figure 4-1). Thus, possible local variations of the stress ratio due to residual stresses in the SENT model were not considered. The calculations presented a better correlation with respect to the experimental values (Figure 5-9). For crack length in the range of $5.08 \mathrm{~mm}$ to less than $13.716 \mathrm{~mm}$, the calculated FCG rates were quite similar to the experimental ones. This supported the idea that for test case 1 with a large stress ratio (nominal $R=0.7$ ), the FCG rate in RSF does not depend on small variations of the effective $R$. The best numerical model in terms of 
accuracy to calculate the FCG rate was based on the SENT model without the crack closure effect and the baseline FCG curve at $R=0.7$.

The results of the FCG rate for the range of crack lengths from $a_{6}=6.096 \mathrm{~mm}$ to $a_{23}=$ 16.764 $\mathrm{mm}$ is presented again in Figure 5-11, but the distribution of the residual stresses at three different fatigue crack lengths is included to analyze their effect in the FCG.

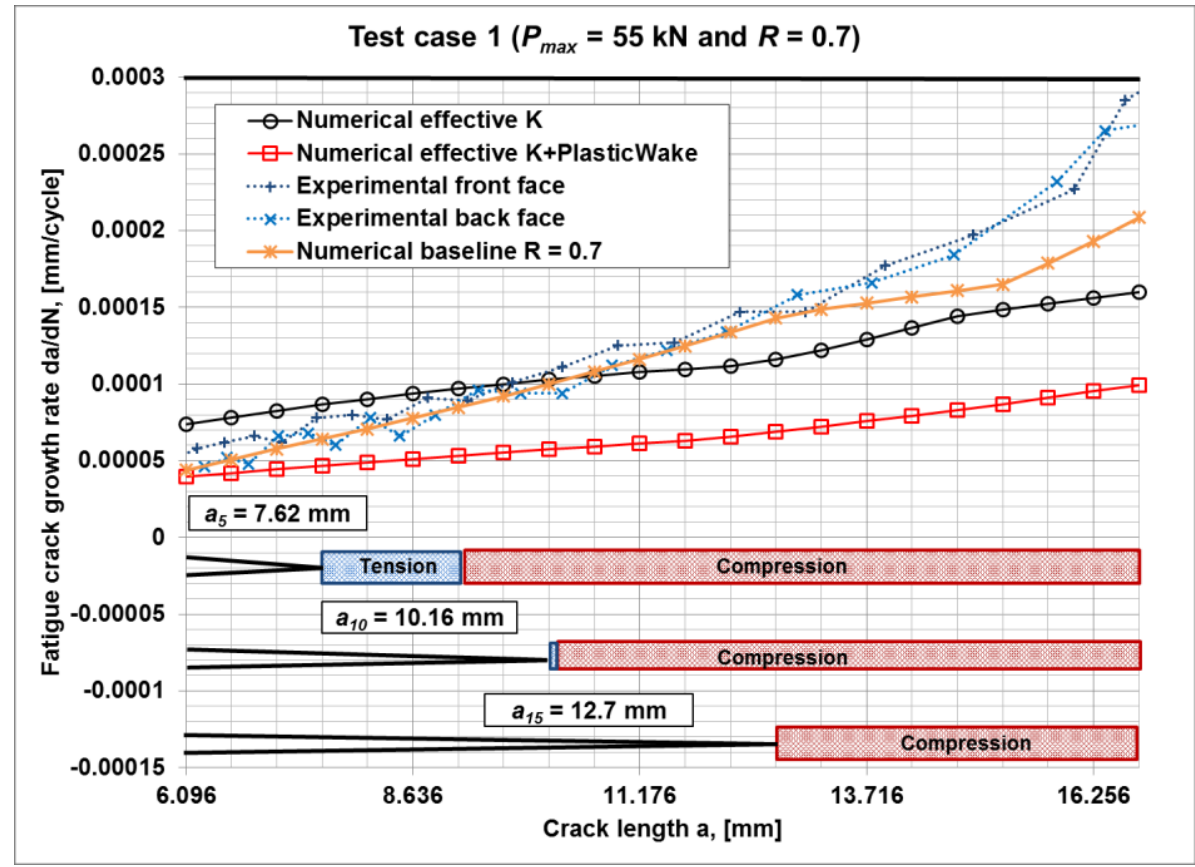

Figure 5-11 Fatigue crack growth rate and residual stresses at different crack lengths for the SENT model with a large stress ratio.

After four-point bending, the initial distribution of the residual stresses in the range of $6.096 \mathrm{~mm}$ to $16.764 \mathrm{~mm}$ was compressive (Figure 5-9b). The redistribution of the residual stresses during the FCG induced a shift from a compressive to a tensile distribution for the residual stresses in front of the crack tip. This shift of sign in the residual stresses was for the range of fatigue crack lengths from $a_{1}=5.588 \mathrm{~mm}$ to $a_{10}=$ 
$10.16 \mathrm{~mm}$ (Figure 5-11). The distribution of residual stresses was compressive for fatigue crack lengths larger than $a_{10}=10.16 \mathrm{~mm}$, which was the case of the fatigue crack length $a_{15}=12.7 \mathrm{~mm}$ observed in Figure 5-11. The FCG rate for the SENT model was increasing for all the range of fatigue crack lengths analyzed. In the case of the fatigue crack lengths from $a_{0}=5.08 \mathrm{~mm}$ to $a_{10}=10.16 \mathrm{~mm}$, the tensile residual stresses contributed to increase the FCG rate. However, the FCG rate for the SENT model with a large stress ratio did not decrease for fatigue crack lengths larger than $a_{10}=10.16 \mathrm{~mm}$ as observed in the numerical and experimental results (Figure 5-11). It seems that the large value of the maximum and minimum applied loads for the SENT model with a large stress ratio was enough to overcome the effect of the compressive residual stresses. Figure 5-12 presents the stress distribution along the FCG plane in the SENT model for fatigue crack lengths $a_{5}=7.62 \mathrm{~mm}, a_{10}=10.16 \mathrm{~mm}$ and $a_{15}=12.7 \mathrm{~mm}$, without the crack closure effect and for the minimum applied cyclic load $\left(P_{\min }=38.5 \mathrm{kN}\right)$. The stress distribution (Figure 5-12) indicated that the crack was completely open, because its magnitude along the crack face was zero for the minimum applied cyclic load. In addition, the fatigue crack lengths produced a stress concentration in front of the crack tip with a maximum value of $533 \mathrm{MPa}$ for $a_{5}=7.62 \mathrm{~mm}, 499 \mathrm{MPa}$ for $a_{10}=10.16 \mathrm{~mm}$ and $458 \mathrm{MPa}$ for $a_{15}=12.7 \mathrm{~mm}$. The plots in Figure 5-12 confirmed that compressive residual stresses in front of the fatigue crack lengths were eliminated by the minimum applied load, as observed for fatigue crack lengths $a_{10}=10.16 \mathrm{~mm}$ and $a_{15}=12.7 \mathrm{~mm}$. 


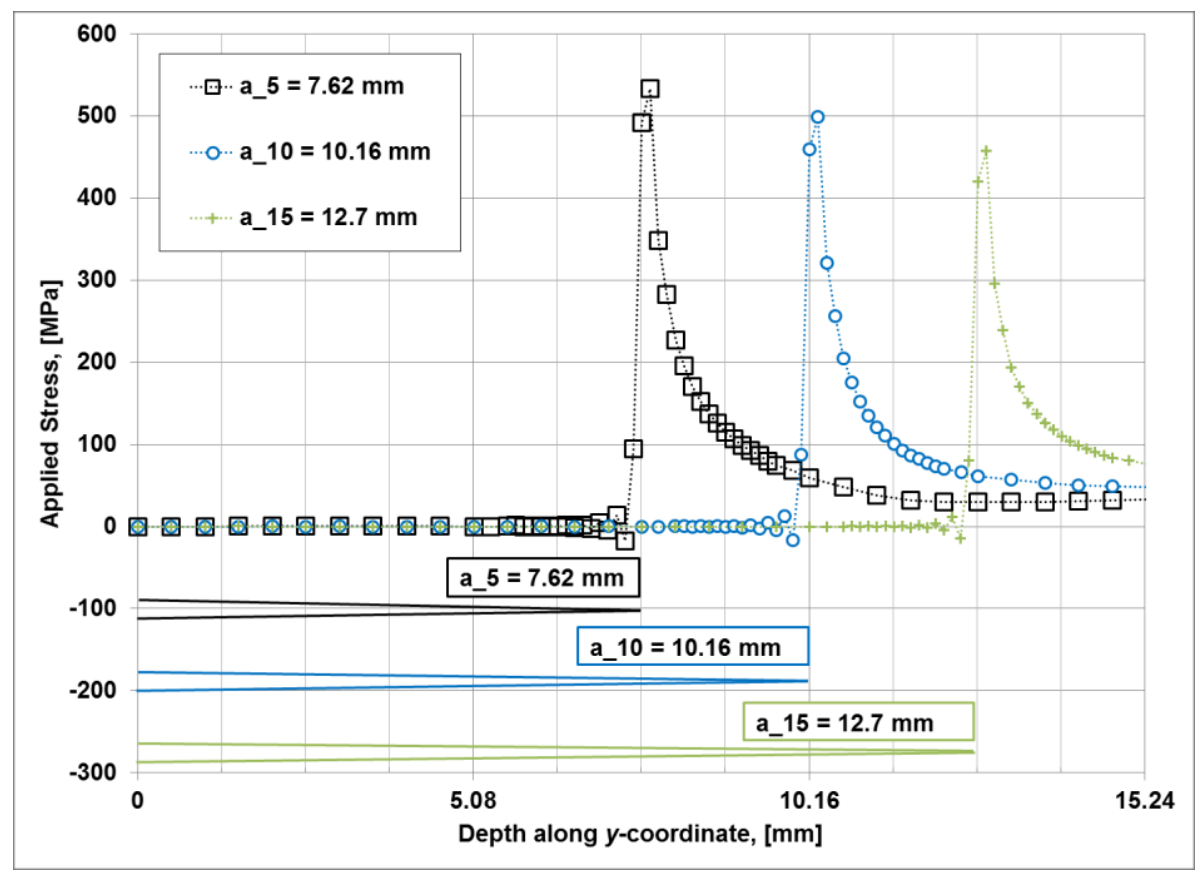

Figure 5-12 The stress distribution (1-D) for fatigue crack lengths $a_{5}=7.62 \mathrm{~mm}, a_{10}=$ $10.16 \mathrm{~mm}$ and $a_{15}=12.7 \mathrm{~mm}$ in the SENT model with the minimum applied cyclic load $\left(P_{\min }=38.5 \mathrm{kN}\right)$ and a large stress ratio (nominal $\left.R=0.7\right)$.

The calculated FCG rates were numerically integrated over each crack length interval to determine the fatigue life curve, i.e. the crack length versus the number of cycles ( $a$ vs. $N)$. Figure 5-13 presents the $a$ vs. $N$ curves for test case $1\left(P_{\max }=55 \mathrm{kN} ; R=0.7\right)$. Again, two plots are shown; Figure 5-13a presents the full range of crack lengths analyzed for specimen C11B10, and Figure 5-13b presents a zoom-in to Figure 5-10a for the range of crack lengths from $a_{0}=5.08 \mathrm{~mm}$ to $a_{20}=15.24 \mathrm{~mm}$ where the residual stresses were in compression. In addition, Figure 5-13 also shows the experimental results of the $a$ vs. $N$ curves for the front- and back-face of the SENT specimen (data in dark-blue and blue colors). 


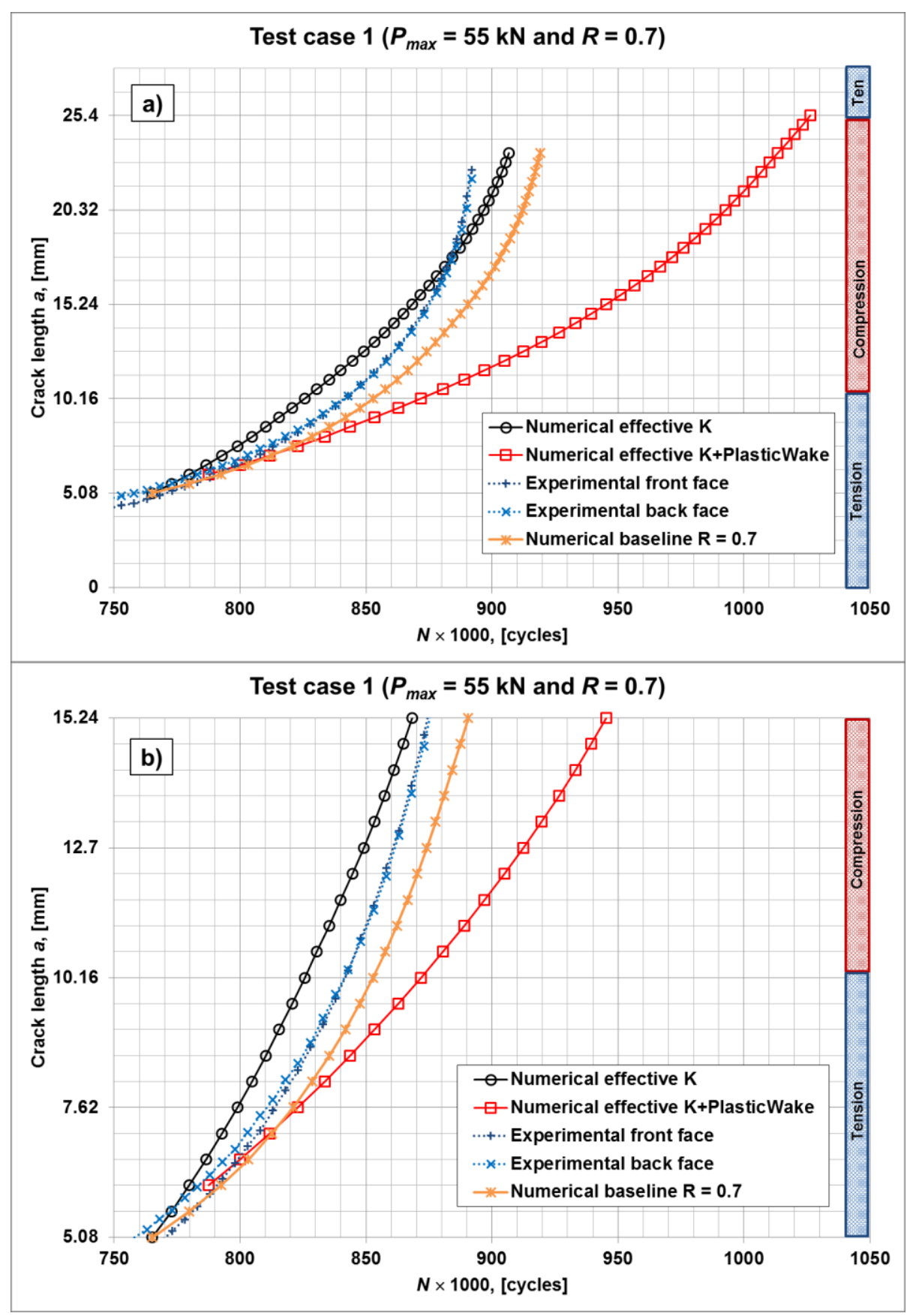

Figure 5-13 Crack length versus number of cycles for test case 1 (C11B10 specimen, $P_{\max }$ $=55 \mathrm{kN}$ and nominal $R=0.7)$.

Vertical bars are included in the plots of Figure 5-13 to specify the distribution of residual stresses in the SENT model with a large stress ratio; the blue color indicates a 
tensile distribution and the red color indicates a compressive distribution. In general, the calculation of the fatigue life by the three SENT models was non-conservative in relation to the experimental measurements. In addition, the SENT models did not produce any extension of the fatigue life because of the RSF in compression. This lack of extension in the fatigue life was verified by the experimental measurements. The most nonconservative result was from the SENT model with the addition of the crack closure and plastic wake effects (Numerical effective $K+$ Plastic Wake, data in red color and rectangle markers; Figure 5-13), because the calculated FCG rates (Figure 5-9) were consistently smaller than the experimental FCG rates for the full range of crack lengths analyzed. The next non-conservative result was from the SENT model without the crack closure effect with the baseline FCG curve at $R=0.7$ (Numerical baseline $R=0.7$, data in orange color with times markers; Figure 5-13), and the less non-conservative result was from the SENT model without the crack closure effect (Numerical effective $K$, data in black color and open circle markers; Figure 5-13). The SENT model with the addition of the crack closure and plastic wake effects over-estimated the fatigue life by a calculationratio of 2.28 , according to the number of cycles in the range of crack lengths analyzed. The calculation-ratio was defined as the calculated fatigue life divided by the experimental fatigue life. In the case of the combination of the SENT model without the crack closure effect and the baseline FCG curve at $R=0.7$, the fatigue life was overestimated by a calculation-ratio of 1.21 . Regarding the SENT model without the crack closure effect, the fatigue life was over-estimated by a calculation-ratio of 1.12. It seemed that the best numerical model was based on the SENT model without the crack closure effect according to the plot of Figure 5-13 and the calculation-ratio of 1.12. 
However, the calculation of the fatigue life was based on a numerical integration of the calculated FCG rates. As a result, overestimations of the FCG rate can be balanced by underestimations of the FCG rate as in the case of the fatigue life calculated by the SENT model without the crack closure effect. As well, a consistent over-prediction or underprediction will produce fatigue life curves with a large offset with respect to the experimental $a$ vs. $N$ curves. For instance, the fatigue life ( $a$ vs. $N$ curve) determined by the SENT model with the plastic wake effect presented a large offset with respect to the experimental measurements (Figure 5-13). Figure 5-13b presented the fatigue life for the first-half of the crack length configurations $(5.08-15.24 \mathrm{~mm})$. The SENT model without the crack closure effect under-estimated the fatigue life by a calculation-ratio of 0.94 , according to the number of cycles in the range of crack lengths of Figure 5-13b (5.08$15.24 \mathrm{~mm}$ ). Therefore, the evaluation of the SENT models based on the plot of the fatigue life and the associated calculation-ratio is an average metric, because the fatigue life calculations are a function of the calculated FCG rates. The best parameter to analyze the accuracy of the SENT models is the plot of the FCG rate as a function of the crack length.

\subsection{Fatigue crack growth rate and fatigue life calculations for test case 2 (nominal $\boldsymbol{R}=\mathbf{0 . 0 5})$}

The conditions of the FCG analysis for test case 2 were set at a low stress ratio (nominal $R=0.05$ ) with a maximum applied cyclic load of $35 \mathrm{kN}$. These conditions corresponded to the specimen C3B5 (Table 4-1) with residual stresses previously introduced by four- 
point bending. ABAQUS ${ }^{\mathrm{TM}}$ was used to simulate the FCG process for test case 2. A displacement correlation method was used to calculate the effective $K_{\max }$ and $K_{\min }$ produced by the SENT model developed in ABAQUSTM. Three SENT models were developed: 1) without the crack closure effect, 2) with the crack closure effect, and 3) with the addition of the crack closure and plastic wake effects. According to the SENT models, the effective $\Delta K$ and $R$ were determined without the crack closure effect (Figure 5-2), with the crack closure effect (Figure 5-4) and with the addition of the crack closure and plastic wake effects (Figure 5-7). Similarly to test case 1, the FCG rate for test case 2 was calculated as a function of the effective $\Delta K$ and $R$ from baseline FCG curves ( $d a / d N$ vs $\Delta \mathrm{K}$ ) for the base material (7050-T7451). The Harter T-method was used to interpolate between experimental baselines FCG curves for 7050-T7451 aluminum alloy (Figure $4-1$ ), in order to calculate new baseline $d a / d N$ vs. $\Delta K$ curves for stress ratios of interest as shown in Figure 4-3. The results of the FCG rate as a function of the fatigue crack length are shown in Figure 5-14 for test case 2. The plots present the results from the FCG model according to three SENT models: 1) without the crack closure effect (Numerical effective $K$; data in black color and open circle markers), 2) with the crack closure effect (Numerical effective $K+$ CrackClosure; data in green color and asterisk markers), and 3) with the addition of the crack closure and plastic wake effects (Numerical effective $K+$ CrackClosure + PlasticWake; data in red color and rectangle markers). In addition, the experimental results of the FCG rate are also shown in Figure 5-14. The experimental results are shown for the front-face (data in dark blue color and plus markers) and backface (data in blue color and multiplication markers) of the SENT specimen of 7050T7451. 


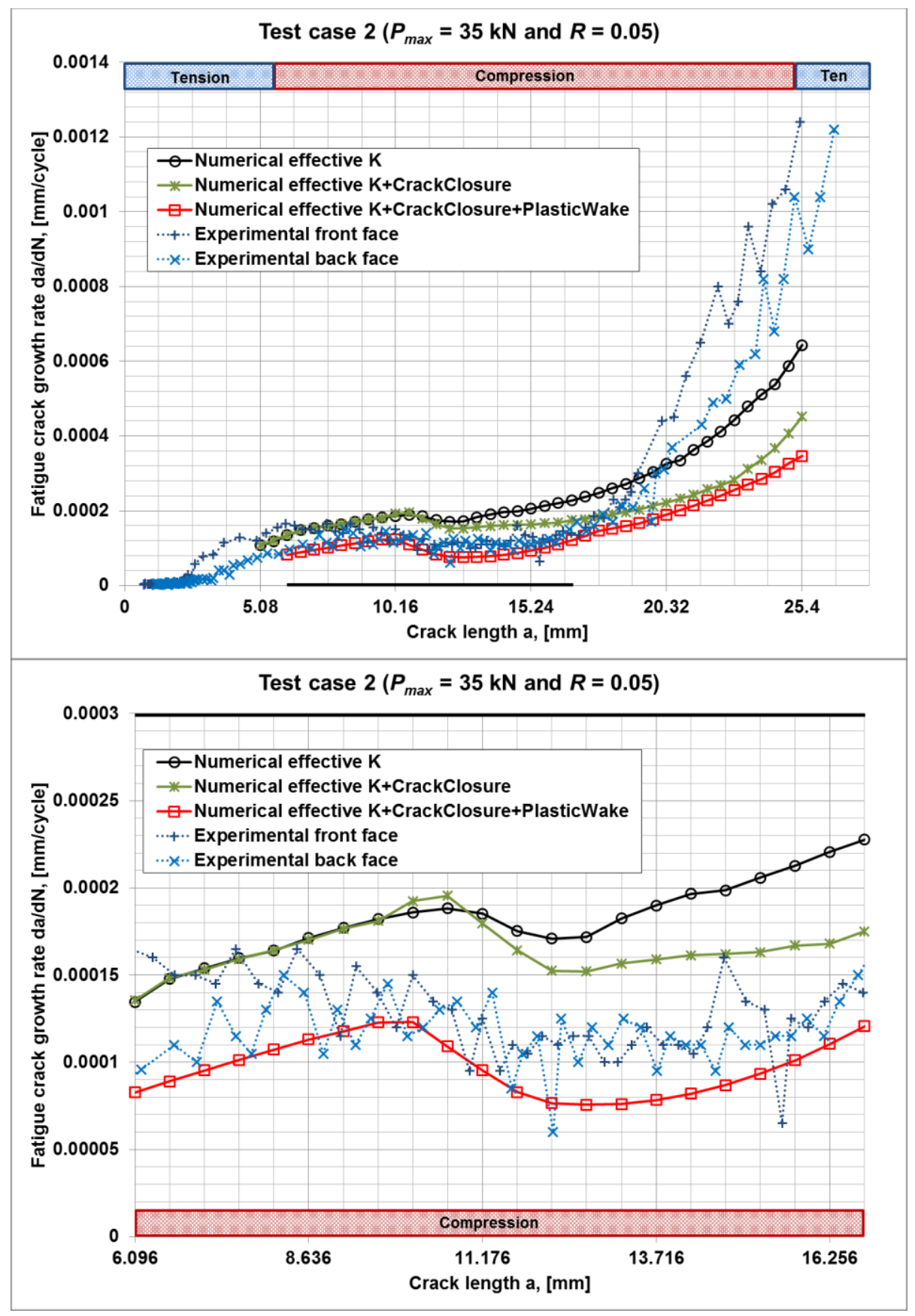

Figure 5-14 Fatigue crack growth rate as a function of the crack length for test case 2

(C5B3 specimen, $P_{\max }=35 \mathrm{kN}$ and $R=0.05$ ): a) Full range of crack lengths, and b) crack lengths $a_{6}=6.096 \mathrm{~mm}$ to $a_{23}=16.764 \mathrm{~mm}$ that correspond to stage II of the FCG curve. 
Two plots are shown in Figure 5-14; the full range of fatigue crack lengths for specimen C3B5 is shown in Figure 5-14a, and a zoom-in to Figure 5-14a for the range of crack lengths from $a_{2}=6.096 \mathrm{~mm}$ to $a_{23}=16.764 \mathrm{~mm}$ is shown in Figure 5-14b. This zoom-in plot corresponds to the stage II of the FCG curve. Horizontal bars are included in the plots of Figure 5-14 to specify the distribution of the initial residual stresses produced by the four-point bending test in the specimen C3B5. The blue color in the bar indicates a tensile stress and the red color indicates a compressive stress.

In general, the predictions based on the finite element model without the crack closure (Numerical effective $K$; Figure 5-14) presented the largest FCG rates, followed by the predictions based on the finite element model with the crack closure effect (Numerical effective $K+$ CrackClosure; Figure 5-14); the lowest FCG rates were predicted by the finite element model with the addition of the crack closure and plastic wake effects (Numerical effective $K+$ CrackClosure + PlasticWake; Figure 5-14). The crack closure effect was not present over the fatigue crack lengths from $a_{0}=5.08 \mathrm{~mm}$ to $a_{11}=10.668$ $\mathrm{mm}$ according to the SENT models with and without the crack closure effect. Therefore, the results of the FCG rate were identical and the crack was completely open for this range of fatigue crack lengths $\left(a_{0}=5.08 \mathrm{~mm}\right.$ to $\left.a_{11}=10.668 \mathrm{~mm}\right)$. The SENT models with and without the crack closure effect exhibited a decrease of the FCG rate for fatigue crack lengths larger than $a_{11}=10.668 \mathrm{~mm}$. In the case of the SENT model without the crack closure effect, the retardation of FCG rate was in the range of fatigue crack lengths from $a_{11}=10.668 \mathrm{~mm}$ to $a_{16}=13.716 \mathrm{~mm}$. The SENT model with the crack closure effect exhibited the FCG retardation for the range of fatigue crack lengths from $a_{11}=$ $10.668 \mathrm{~mm}$ to $a_{28}=19.304 \mathrm{~mm}$. A retardation effect was also captured by the SENT 
model with the addition of the crack closure and plastic wake effects for the range of fatigue crack lengths from $a_{9}=9.652 \mathrm{~mm}$ to $a_{23}=16.764 \mathrm{~mm}$. The retardation effect was captured by the three numerical models, but the best correlation with respect to the experimental measurements of the FCG rate resulted from the addition of the crack closure and plastic wake effects (Figure 5-14b). The decrease in the FCG rate was caused by the presence of compressive residual stresses in the specimen C5B3. However, the initial distribution of compressive residual stresses in the specimen C5B3 (after the fourpoint bending test) were for the range of length from $5.588 \mathrm{~mm}$ to $16.764 \mathrm{~mm}$ (Figure $5-14 b)$, but the decrease in the growth rate was produced when the fatigue crack length was larger than $10 \mathrm{~mm}$ as observed in the experimental results. Moreover, the FCG rates were gradually increased during the interval of crack lengths $a_{0}=5.08$ to $a_{11}=10.668$ $\mathrm{mm}$ in spite of the compressive distribution of residual stresses after the four-point bending. Figure 5-15 presents again the results of the FCG rate for the range of crack lengths from $a_{6}=6.096 \mathrm{~mm}$ to $a_{23}=16.764 \mathrm{~mm}$, but the distribution of residual stresses because of the FCG is included for three crack lengths. The distribution of residual stresses in Figure 5-15 was determined from the SENT model with the addition of the crack closure and plastic wake effects. 


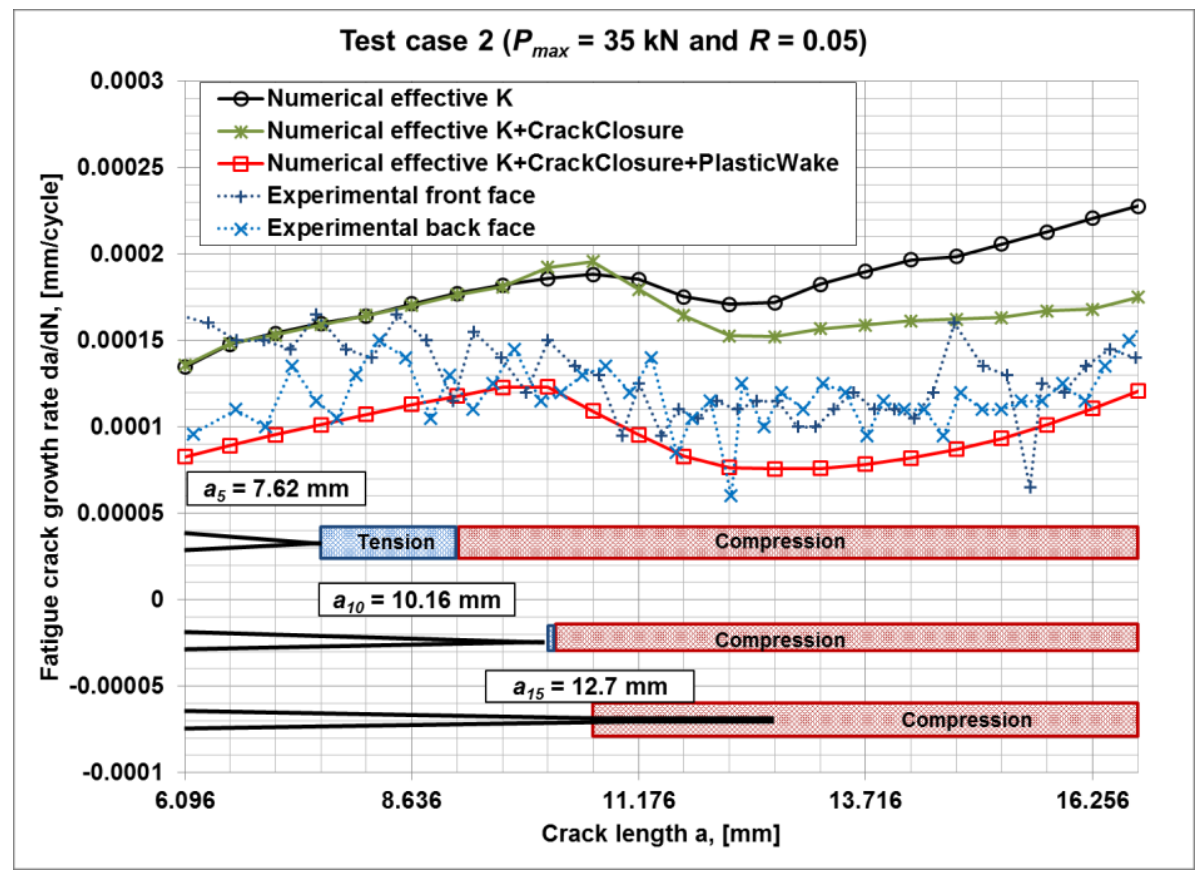

Figure 5-15 Fatigue crack growth rate and residual stresses at different crack lengths for the SENT model with a low stress ratio.

As observed in Figure 5-15, the redistribution of residual stresses caused the decrease of the FCG rate after the fatigue crack length $a_{11}=10.668$. The fatigue crack lengths $a_{5}=$ $7.62 \mathrm{~mm}$ and $a_{10}=10.16 \mathrm{~mm}$ presented tensile residual stresses in front of their crack tip as observed in Figure 5-15. However, both lengths $\left(a_{5}=7.62 \mathrm{~mm}\right.$ and $\left.a_{10}=10.16 \mathrm{~mm}\right)$ were in compression after the four-point bending (Figure 5-14b), but the redistribution due to FCG caused a shift from compressive into tensile residual stresses. The fatigue crack length $a_{15}=12.7 \mathrm{~mm}$ presented compressive residual stresses in front and behind of its crack tip. Figure 5-16 presents the stress distribution along the FCG plane in the SENT model for fatigue crack lengths $a_{5}=7.62 \mathrm{~mm}, a_{10}=10.16 \mathrm{~mm}$ and $a_{15}=12.7 \mathrm{~mm}$, with the addition of the crack closure and plastic wake effects and for the minimum applied cyclic load $\left(P_{\min }=1.75 \mathrm{kN}\right)$. 


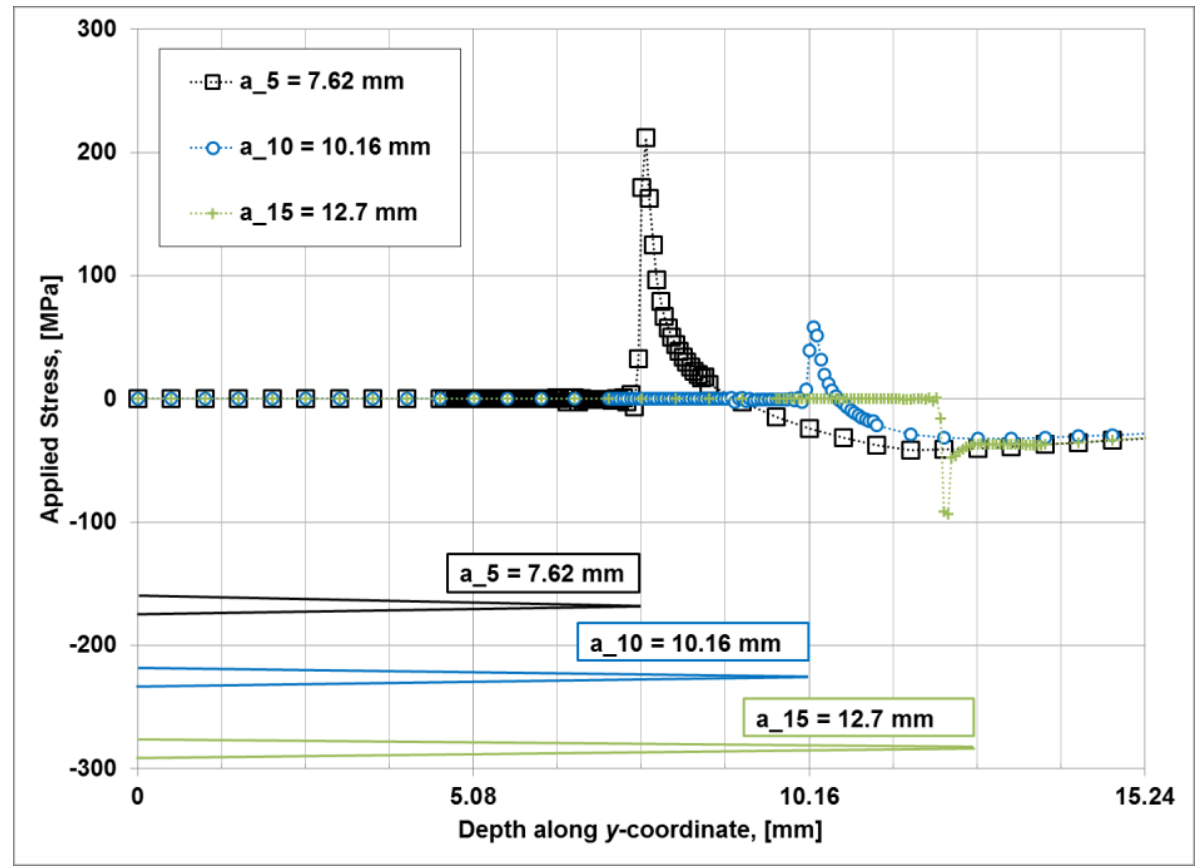

Figure 5-16 The stress distribution (1-D) for fatigue crack lengths $a_{5}=7.62 \mathrm{~mm}, a_{10}=$ $10.16 \mathrm{~mm}$ and $a_{15}=12.7 \mathrm{~mm}$ in the SENT model with the minimum applied cyclic load $\left(P_{\min }=1.75 \mathrm{kN}\right)$ and a low stress ratio (nominal $\left.R=0.05\right)$.

The crack was completely open for fatigue crack lengths $a_{5}=7.62 \mathrm{~mm}$ and $a_{10}=10.16$ $\mathrm{mm}$, because the stresses were zero behind the crack tip (Figure 5-16) for the minimum applied load $\left(P_{\text {min }}=1.75 \mathrm{kN}\right)$. These fatigue crack lengths produced a stress concentration in front of the crack tip with a maximum value of $212 \mathrm{MPa}$ for $a_{5}=7.62$ $\mathrm{mm}$ and $58 \mathrm{MPa}$ for $a_{10}=10.16 \mathrm{~mm}$. On the contrary, the fatigue crack length $a_{15}=12.7$ mm was partially closed at the crack tip, because the stresses behind the crack tip were negative for a length of approximately $2 \mathrm{~mm}$ (Figure 5-16). In addition, the stresses in front of the crack tip for the crack length $a_{15}=12.7 \mathrm{~mm}$ were also negative. The negative stresses behind the crack tip induced a crack closure effect, and in conjunction with the 
negative stresses in front of the crack tip produced a decrease in the FCG rate (Figure $5-15)$

Figure 5-17 presents the crack length versus the number of cycles ( $a$ vs. $N$ ) according to the FCG model and the experimental study for the test case 2 with a low stress ratio $\left(P_{\max }\right.$ $=35 \mathrm{kN}$ and nominal $R=0.05)$. Vertial bars are included in the plot of Figure 5-17 to show the distribution of residual stresses in the SENT model due to FCG; the blue color indicates a tensile distribution and the red color indicates a compressive distribution.

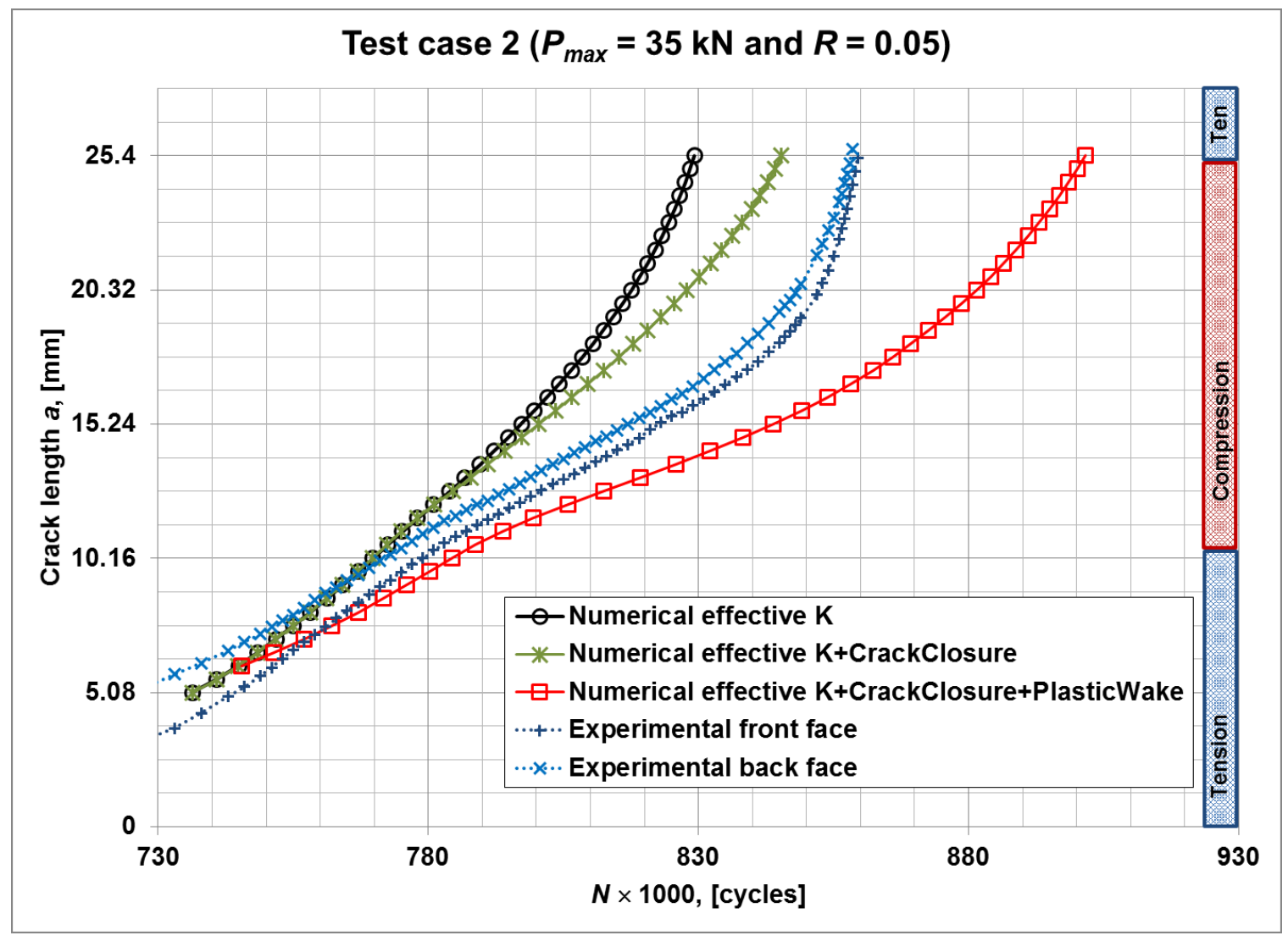

Figure 5-17 Crack length versus number of cycles for test case 2 (C5B3 specimen, $P_{\max }=$ $35 \mathrm{kN}$ and nominal $R=0.05)$. 
The calculation of the fatigue life ( $a$ vs $N$ ) was conservative with respect to the experimental measurements according to the SENT models without the crack closure effect (Numerical effective $K$, data in black color and open circle markers; Figure 5-17) and with the crack closure effect (Numerical effective $K+$ CrackClosure, data in green color and asterisk markers; Figure 5-17). The most conservative result was from the SENT model without the crack closure effect. Meanwhile, the fatigue life determined by the SENT model with the addition of the crack closure and plastic wake effects (Numerical effective $K+$ CrackClosure + PlasticWake, data in red color and rectangle markers) was non-conservative with respect to the experimental measurements. The experimental measurements in Figure 5-17 are shown for the front-face (data in dark blue color and plus markers) and back-face (data in blue color and times markers) of the SENT specimen of 7050-T7451. According to the experimental measurements, an extension of the fatigue life over the range of fatigue crack lengths from $10 \mathrm{~mm}$ to $15 \mathrm{~mm}$ was observed. This extension of the fatigue life for specimen C5B3 was the result of the decrease in the FCG rate. The three SENT models produced a decrease of the FCG rate (Figure 5-14), although only the results of the fatigue life from the SENT model with the addition of the crack closure and plastic wake effects presented an extension of the fatigue life over the fatigue crack lengths from approximately $\mathrm{a}_{11}=10.668 \mathrm{~mm}$ to $\mathrm{a}_{20}=$ 15.24 mm, (Figure 5-17). For the number of cycles in the interval of crack lengths analyzed, the fatigue life was under-estimated by 0.88 and 0.75 according to the SENT model with and without the crack closure effect, respectively. In the case of the SENT model with the addition of the crack closure and plastic wake effects, the fatigue life was over-estimated by 1.26 . 


\subsection{Fatigue crack growth rate and fatigue life calculations for test case 3 (nominal $R=-1)$}

The conditions of the FCG analysis for test case 3 were a negative stress ratio $R$ with a nominal value of -1 and a maximum applied cyclic load $P_{\max }$ of $35 \mathrm{kN}$. These conditions corresponded to the specimen C10B8 according to the test matrix presented in Table 4-1. The results of the FCG rate according to the SENT models and the experimental study for the test case 3 are presented in Figure 5-18. The results of the FCG rate for the full range of crack lengths analyzed is shown in Figure 5-18a, while Figure 5-18b presents a zoomin to Figure 5-18a for the range of crack lengths $a_{6}=6.096 \mathrm{~mm}$ to $a_{23}=16.764 \mathrm{~mm}$. The plots also show the stress distribution of the initial residual stresses produced by the fourpoint bending test in the C10B8 specimen. Horizontal bars in blue color indicate tensile residual stresses and compressive residual stresses are indicated by a red color in the bar. The experimental results of the FCG rate are shown for the front-face (data in dark-blue color and plus markers) and back-face (data in blue color and times markers) of the C10B8 specimen. These experimental results show a retardation effect in the FCG rate for crack lengths in the range of $7 \mathrm{~mm}$ to $17 \mathrm{~mm}$ approximately (Figure 5-18b). The FCG rate calculations by the SENT model with the addition of the crack closure and plastic wake effects (Numerical effective $K+$ CrackClosure + PlasticWake, data in red color and rectangle markers; Figure 5-18) were smaller than the calculations of the growth rate using the SENT model with the addition of the crack closure effect (Numerical effective $K+$ CrackClosure, data in green color and asterisk markers; Figure 5-18). 


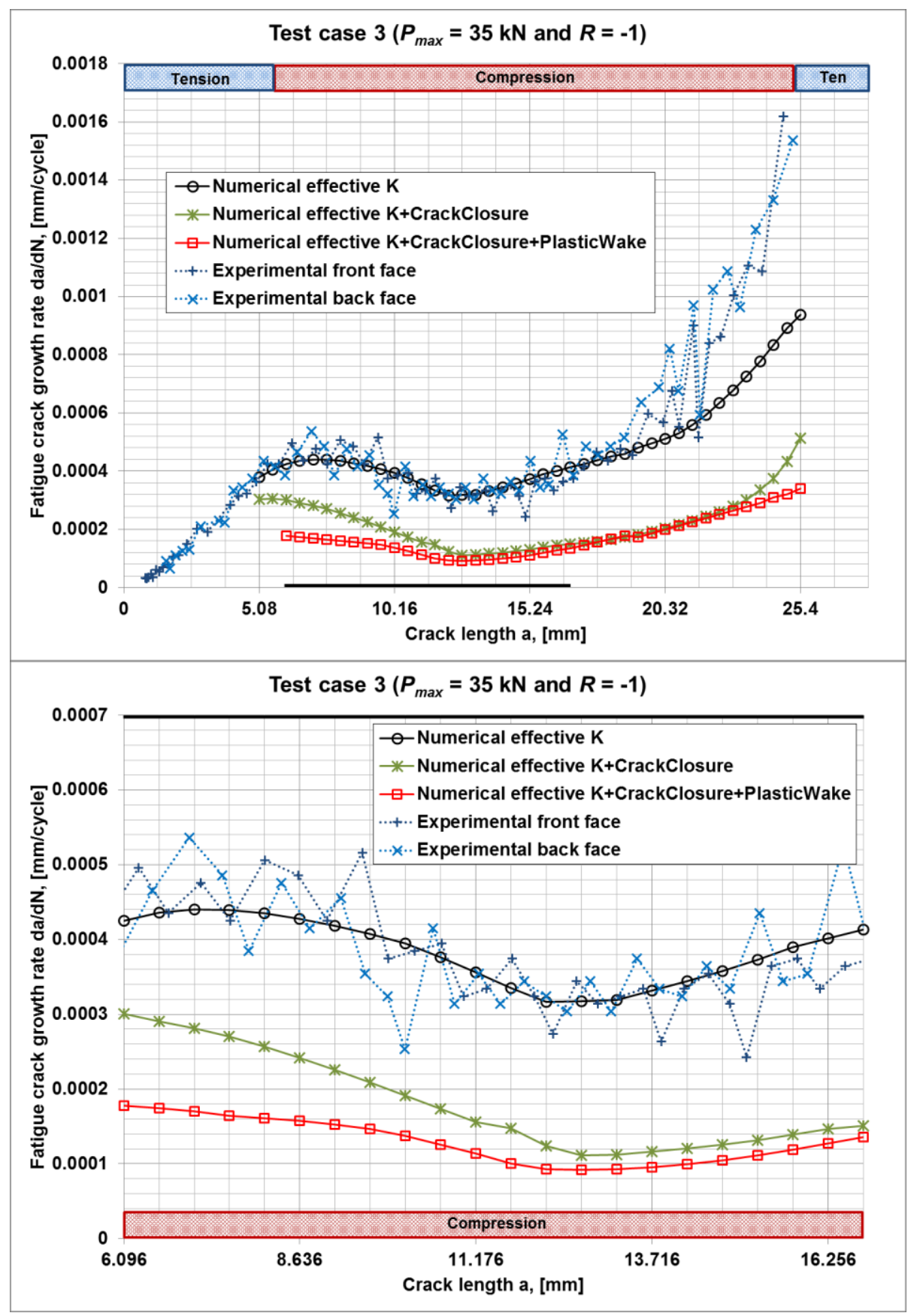

Figure 5-18 Fatigue crack growth rate as a function of the crack length for test case 3

(C10B8 specimen, $P_{\max }=35 \mathrm{kN}$ and nominal $R=-1$ ): a) Full range of crack lengths, and

b) crack lengths $a_{6}=6.096 \mathrm{~mm}$ to $a_{23}=16.764 \mathrm{~mm}$. 
Both SENT models (Numerical effective $K+$ CrackClosure + PlasticWake and Numerical effective $K+$ CrackClosure) underestimated the FCG rate for specimen C10B8 according with the experimental measurements. On the contrary, the results of the FCG rate were accurately determined by the SENT model without the crack closure effect (Numerical effective $K$, data in black color and open circle markers; Figure 5-18). In addition, the retardation effect in the FCG rate was accurately determined by the SENT model without the crack closure effect (Figure 5-18b). This retardation effect was caused by the presence of the compressive residual stresses in the SENT model. The retardation effect was overestimated by the SENT models with the crack closure, and with the addition of the crack closure and plastic wake effects. It seems that the crack closure effect should not be considered for the SENT model with a negative stress ratio $R$ with a nominal value of -1 , because the effective stress ratio was limited to a zero value (Figure 5-5 and Figure 5-8). The calculations of the effective stress ratio from the SENT model without the crack closure effect were not limited to a zero value and presented negative values of the effective $R$ (Figure 5-3). This negative values of the effective $R$ were responsible for the accurate calculation of the FCG rate in the SENT model with residual stresses. Figure 5-19 presents again the results of the FCG rate for the range of crack lengths from $a_{6}=6.096 \mathrm{~mm}$ to $a_{23}=16.764 \mathrm{~mm}$, but the distribution of residual stresses produced by the FCG is included for three crack lengths: $a_{5}=7.62 \mathrm{~mm}, a_{10}=$ $10.16 \mathrm{~mm}$ and $a_{14}=12.192 \mathrm{~mm}$. The distribution of residual stresses in Figure 5-19 was determined from the SENT model without the crack closure effect. 


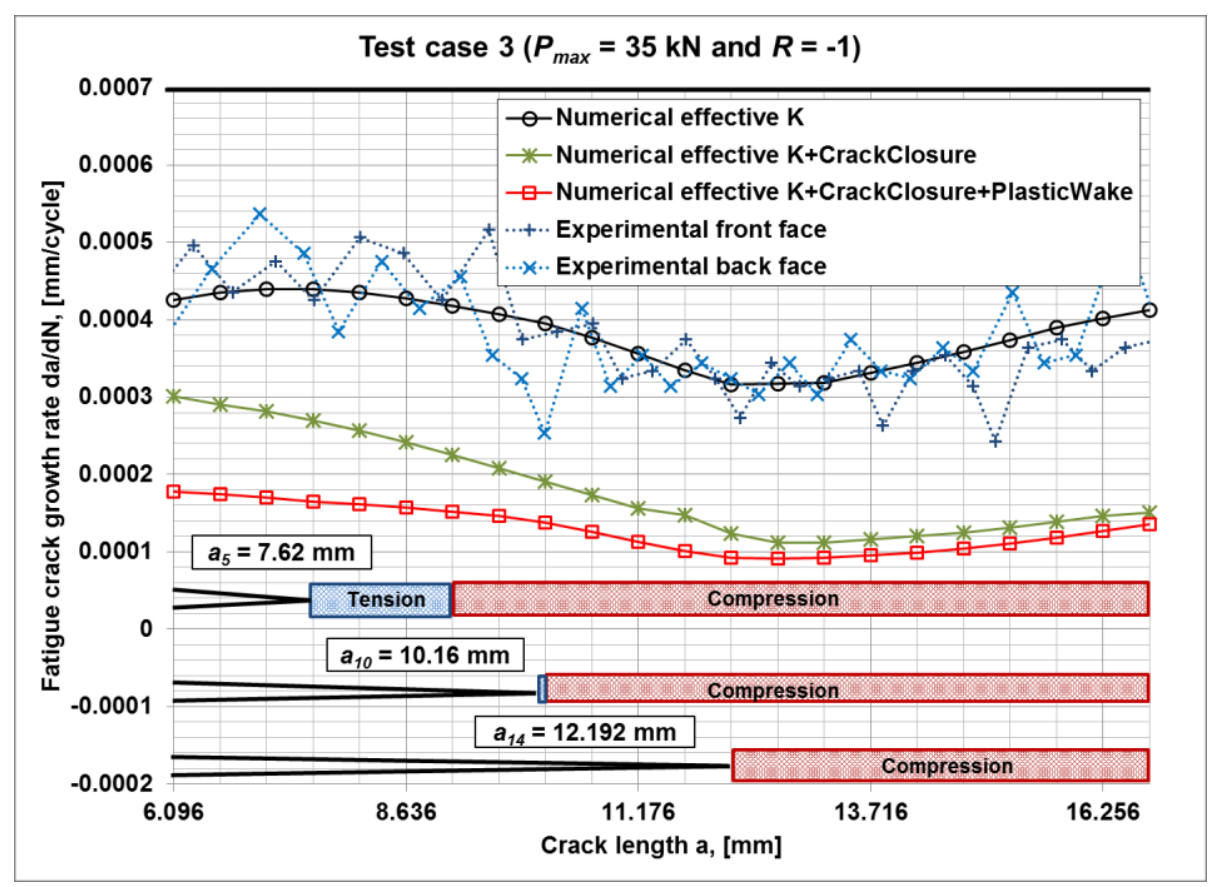

Figure 5-19 Fatigue crack growth rate and residual stresses at different crack lengths for the SENT model with a negative stress ratio.

The initial residual stresses in compression for specimen C10B8 produced by the fourpoint bending test (Figure 5-18) were redistributed into tensile residual stresses by the FCG for the range of crack lengths $a_{1}=5.588 \mathrm{~mm}$ to $a_{10}=10.16 \mathrm{~mm}$ (Figure 5-19). These redistributed residual stresses corresponded to a zero applied load. In the case of the minimum applied load $\left(P_{\min }=-35 \mathrm{kN}\right)$, the stress distribution along the FCG plane in the SENT model without the crack closure effect is presented in Figure 5-20 for fatigue crack lengths $a_{5}=7.62 \mathrm{~mm}, a_{10}=10.16 \mathrm{~mm}$ and $a_{14}=12.192 \mathrm{~mm}$. A stress concentration was found in front of the crack tip with a negative distribution for the minimum applied load to the SENT model without the crack closure effect. The value of the stress concentration was -257 MPa for fatigue crack length $a_{5}=7.62 \mathrm{~mm},-473 \mathrm{MPa}$ for fatigue crack length $a_{10}=10.16 \mathrm{~mm}$ and $-575 \mathrm{MPa}$ for fatigue crack length $a_{14}=12.192 \mathrm{~mm}$. 


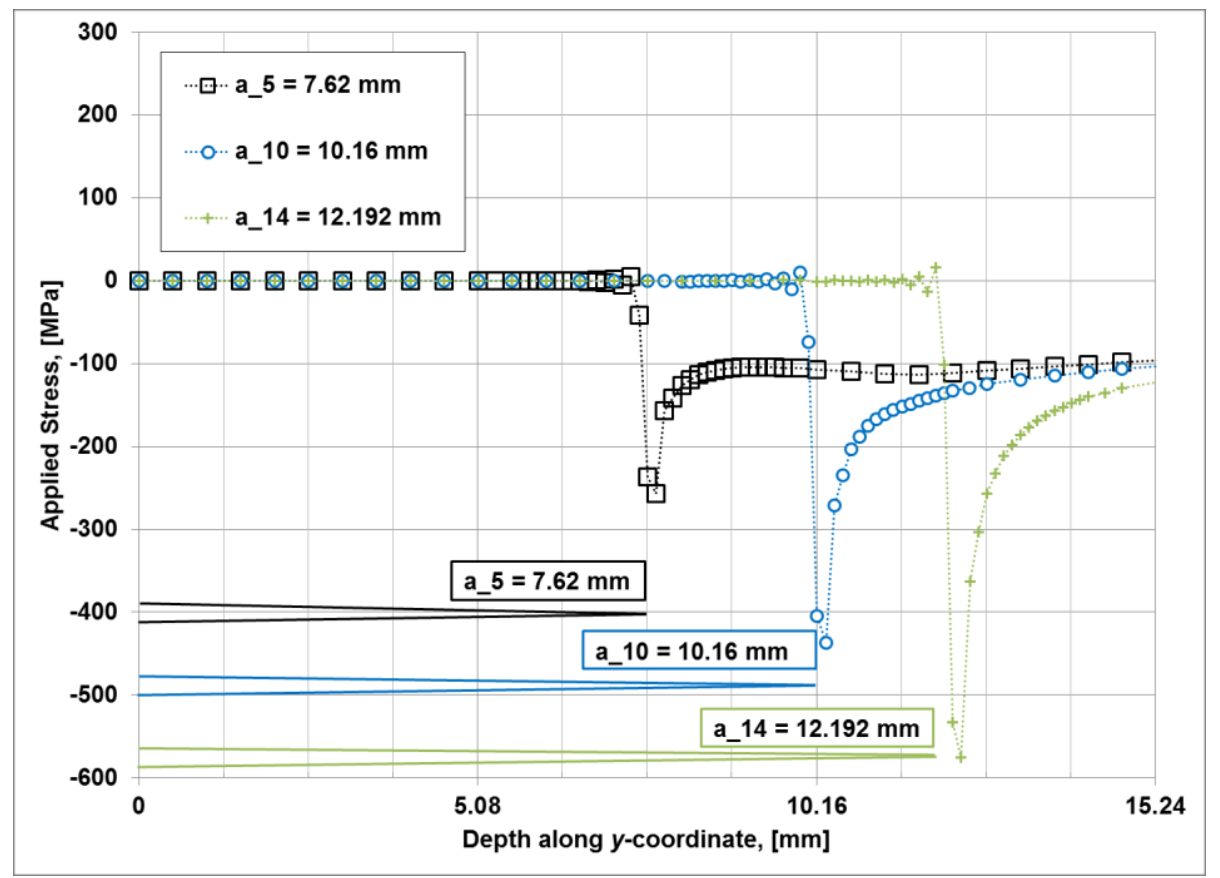

Figure 5-20 The stress distribution (1-D) for fatigue crack lengths $a_{5}=7.62 \mathrm{~mm}, a_{10}=$ $10.16 \mathrm{~mm}$ and $a_{14}=12.192 \mathrm{~mm}$ in the SENT model with the minimum applied cyclic load $\left(P_{\min }=-35 \mathrm{kN}\right)$ and a low stress ratio (nominal $\left.R=0.05\right)$.

Figure 5-21 presents the crack length versus the number of cycles according to the FCG model and the experimental study for test case 3 with a negative stress ratio $\left(P_{\min }=-35\right.$ $\mathrm{kN}, R=-1$ ). The calculations from the SENT model with the crack closure effect (data in green color and asterisk markers; Figure 5-21) and with the addition of the crack closure and plastic wake effects (data in red color and rectangle markers; Figure 5-21) presented a large offset with respect to the experimental measurements. The fatigue life was overestimated by a ratio of 2.53 and 2.93 according to the SENT models with the crack closure and with the addition of the crack closure and plastic wake effects. The SENT model without the crack closure effect (data in black color and open circle markers; 
Figure 5-21) produced the best estimation of the fatigue life for the SENT specimen with residual stresses. The fatigue was over-estimated by a ratio of just 1.08 according to the SENT model without the crack closure effect. The experimental measurements presented an extension of the fatigue life for the range of crack lengths from 10 to $15 \mathrm{~mm}$ approximately. This extension of the fatigue life was accurately calculated by the SENT model without the crack closure effect (Figure 5-21). This extension of the fatigue life was a consequence of the retardation effect in the FCG rate because of the compressive RSF.

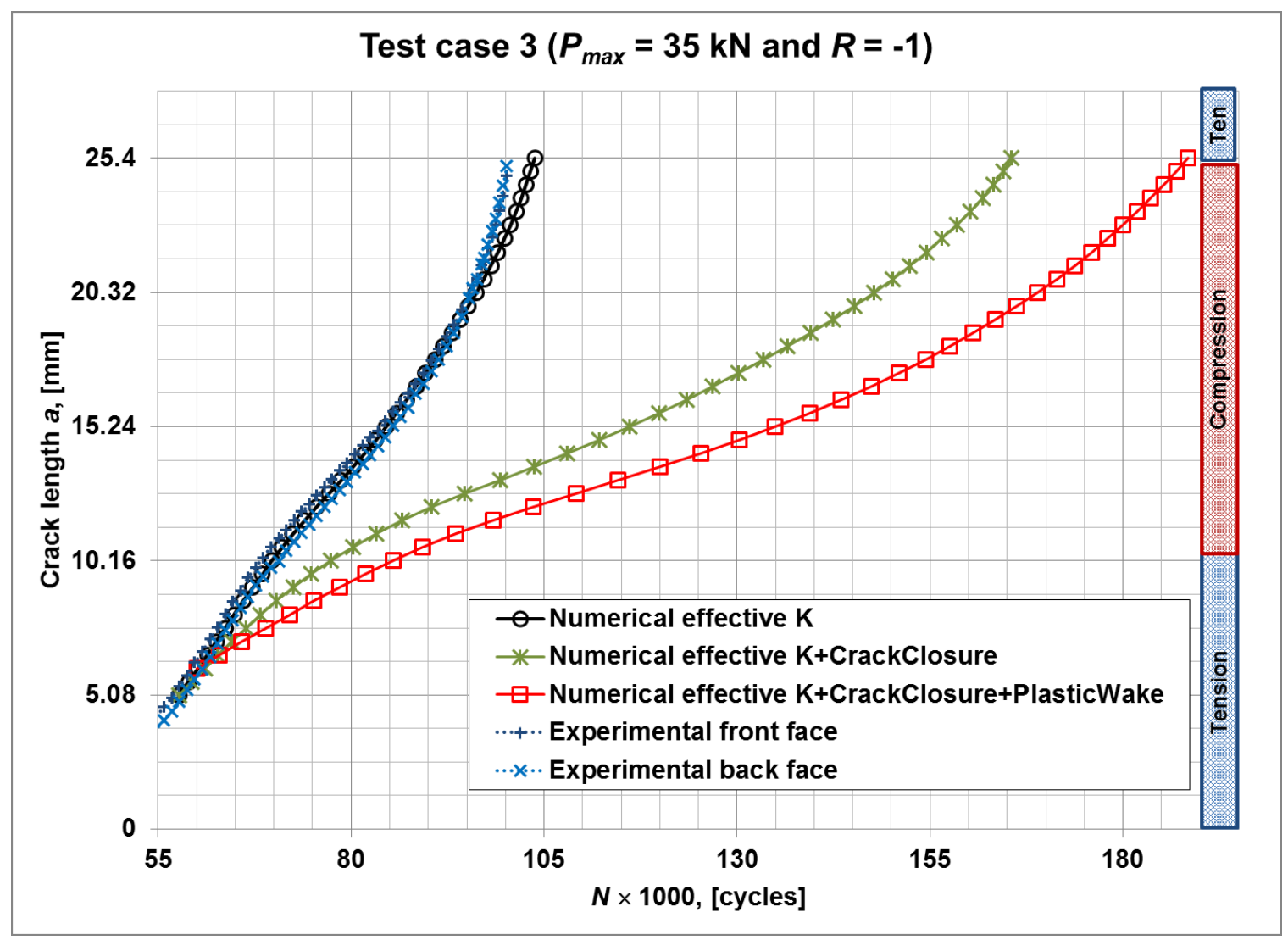

Figure 5-21 Crack length versus number of cycles for test case 3 (C10B8 specimen, $P_{\max }$ $=35 \mathrm{kN}$ and nominal $R=-1$ ). 


\subsection{Summary of the Results}

For the large stress ratio (nominal $R=0.7$ ) used in the FCG analysis of test case 1 (C11B10 specimen), the residual stresses did not induced a retardation effect in the FCG rate as shown by the experimental measurements (Figure 5-9). Also, the results of the FCG model did not show a retardation effect. The best calculation of the FCG rate was plotted with an orange line (Figure 5-9). The results indicated that in the case of large applied stress ratio, the effective stress ratio does not change significantly during FCG even in the presence of RSF. The best estimation of the fatigue life was done by the SENT model without the crack closure effect, because overestimations of the FCG rate for the range of crack lengths from $a_{0}=5.08 \mathrm{~mm}$ to $a_{7}=8.636 \mathrm{~mm}$ (Figure 5-9) were equilibrated by underestimations over the crack lengths from $a_{12}=11.176 \mathrm{~mm}$ to $a_{36}=$ $23.368 \mathrm{~mm}$. The plastic wake effect should not be considered for test case 1 (SENT specimen with residual stresses subjected to a large stress ratio), because the calculated FCG rates were consistently smaller than the experimental measurement of the FCG rate (Figure 5-9).

For the low stress ratio (nominal $R=0.05$ ) used in the FCG analysis of test case 2 (C5B3 specimen), the residual stresses induced a retardation effect in the FCG rate as shown by the experimental measurements (Figure 5-14). This retardation effect was also predicted by the FCG models (Figure 5-14). A prediction ratio was defined as the numerical FCG rate divided by the experimental one as a function of the crack length. This prediction ratio was used to determine the best numerical model for test case 2 with a low stress ratio. Figure 5-22 presents a plot of the prediction ratio for the range of crack lengths 
from $a_{2}=6.096 \mathrm{~mm}$ to $a_{23}=16.764 \mathrm{~mm}$. The experimental FCG rate was defined as the average from the front- and back-face measurements in specimen C5B3 (Figure 5-14).

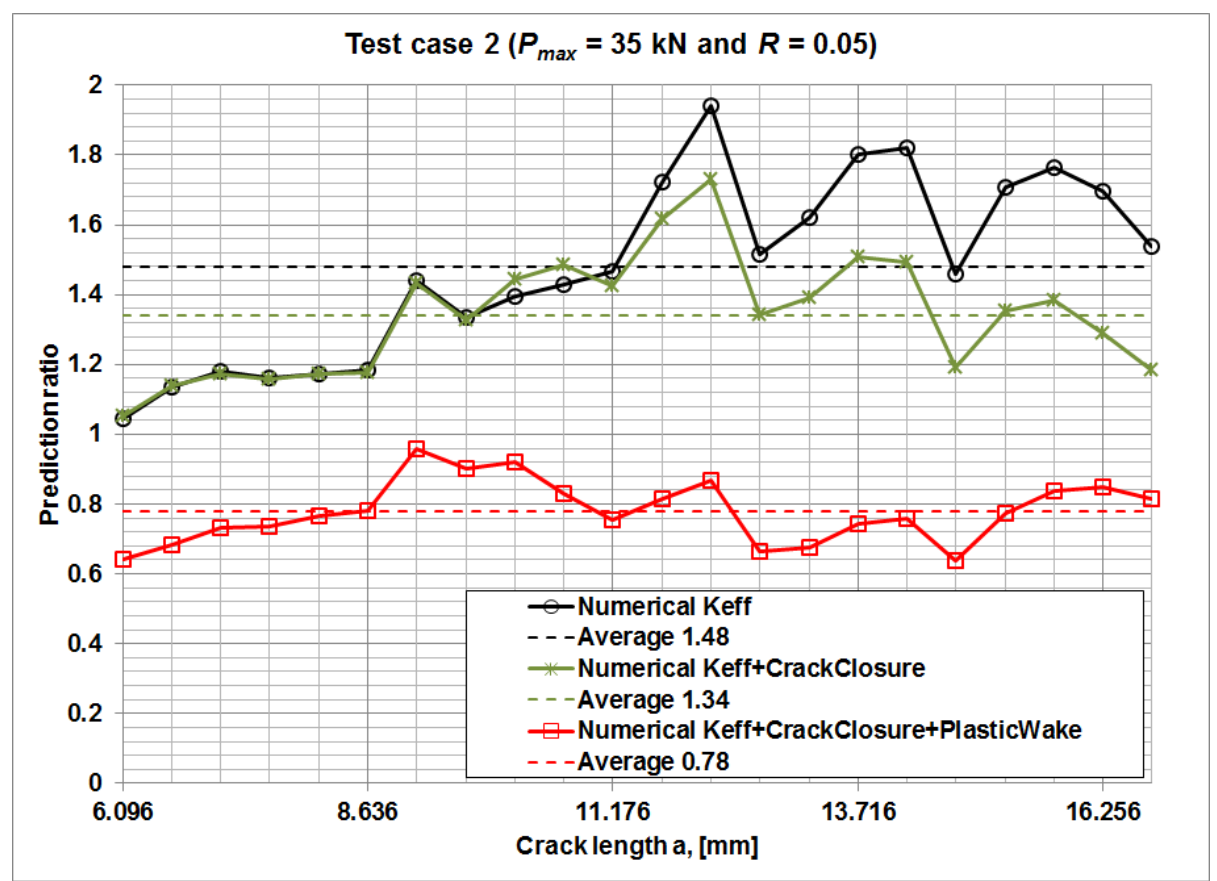

Figure 5-22 Prediction ratio of the fatigue crack growth rate according to the three SENT models.

The prediction ratio according to the SENT model without the crack closure effect is shown in green color and asterisk markers in Figure 5-22; the black color and open circle markers are for the SENT model with the crack closure effect and the red color with rectangle markers was for the SENT model with the addition of the crack closure and plastic wake effects. A dotted line indicates the average prediction ratio according to each SENT model. The value of the average prediction ratio produced by the SENT model without the crack closure effect was 1.34; with the crack closure effect it was 1.48 and with the addition of the crack closure and plastic wake effect it was 0.78 . The SENT 
model with the addition of the crack closure and plastic wake effects produced the best results of the FCG rate with respect to the experimental measurements. However, the best estimation of the fatigue life was done by the SENT model with the crack closure effect, because overestimations of the FCG rate for the initial crack lengths were equilibrated by underestimations during the final crack lengths (Figure 5-17). In the three SENT models, the worst correlation of the FCG rate with respect to the experimental measurements was found when the crack length was larger than approximately $20 \mathrm{~mm}$ (Figure 5-14). This lack of accuracy was a consequence of the FCG model (equation 13), which is limited to analyze the linear region of the FCG curve for the base material. The final region of the FCG curve presents an unstable regimen for the FCG rate, which is highly dependent on microstructural conditions. The FCG model developed in this research (Chapter 4) did not consider microstructural parameters.

Figure 5-23 presents plots of the crack length profile during the FCG test simulation, according to the SENT model with the addition of the crack closure and plastic wake effects. The profiles are presented for crack lengths $a_{0}=5.08 \mathrm{~mm}, a_{5}=7.62 \mathrm{~mm}, a_{15}=$ $12.7 \mathrm{~mm}, a_{25}=17.78 \mathrm{~mm}$ and $a_{35}=22.86 \mathrm{~mm}$ at maximum and minimum cyclic loads (Figure 5-23a and Figure 5-23b). As a reference for the crack length and face, a contour plot is also presented in Figure 5-23a for crack length $a_{0}=5.08 \mathrm{~mm}$. The crack length was along the $y$-direction and the crack face was opened along the $x$-direction. 

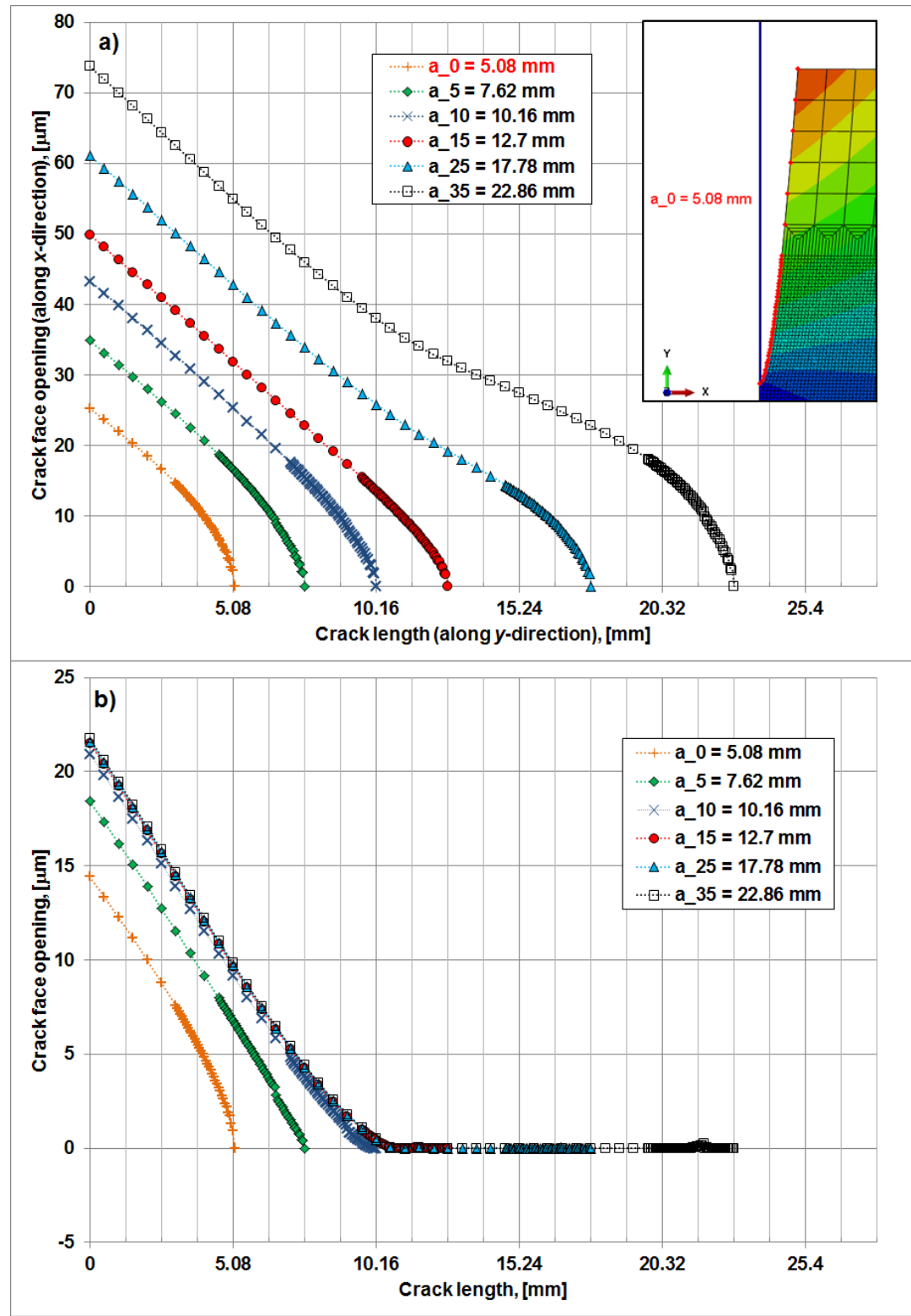

Figure 5-23 Crack length profile during fatigue crack growth test simulation of test case 2 with a low stress ratio: a) Maximum applied cyclic load $\left(P_{\max }=35 \mathrm{kN}\right)$ and b) minimum applied cyclic load $\left(P_{\min }=1.75 \mathrm{kN}\right)$, with closure and plastic wake effects.

It was verified that for the fatigue crack with a length smaller than $10.16 \mathrm{~mm}\left(a_{n}<10.16\right.$ $\mathrm{mm}$ ), the crack in the SENT model was completely open for the maximum and minimum applied loads (Figure 5-23). On the contrary, the fatigue crack was completely closed for 
the fatigue crack with a length larger than $10.16 \mathrm{~mm}$. Besides, a blunted crack tip was resolved from the SENT model with the addition of the crack closure and plastic wake effects (Figure 5-23a) for the maximum applied cyclic load. This blunted crack tip is characterized for having a finite radius rather than a sharp tip. Figure 5-24 presents the crack front profile during the FCG simulation of test case 2 with a low stress ratio. Several crack fronts are shown in Figure 5-24 according to the number of cycles calculated by the FCG model. The crack front was defined by the shape of the crack along the thickness of the SENT model.

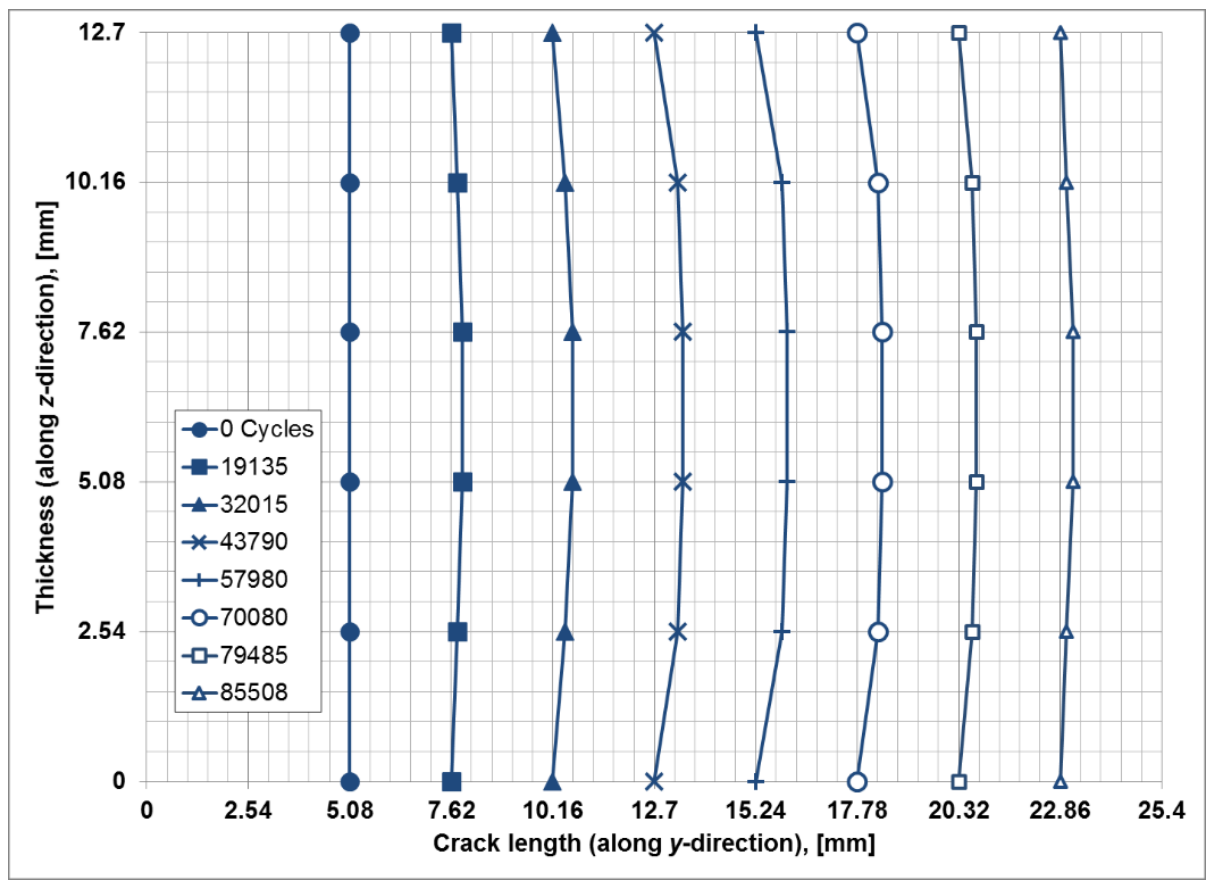

Figure 5-24 Crack front profile during the fatigue crack growth test simulation of test case 2 with a low stress ratio.

The crack length $a_{0}=5.08 \mathrm{~mm}$ in the SENT model was defined with a linear crack front, but the crack front became curved during the FCG simulation. The crack front in the 
SENT model became curved, because the FCG rate was larger for the internal nodes along the thickness of the SENT model. For instance, the fatigue crack length $a_{15}=12.7$ $\mathrm{mm}$ presented a FCG rate of $0.00016 \mathrm{~mm} / \mathrm{cycle}$ for the nodes on the surface of the SENT model, and a FCG rate of $0.00019 \mathrm{~mm} / \mathrm{cycle}$ for the internal nodes along the thickness of the SENT model. Thus, the crack front curvature was a consequence of the different FCG rates between the surface and the internal nodes of the SENT model.

The SENT specimen with a crack length $a_{0}=5.08 \mathrm{~mm}$ and subjected to a maximum applied load of $35 \mathrm{kN}$ (test case 2) presented a plastic zone at the crack tip with a radius of $0.068 \mathrm{~mm}$, according to the Irwin equation. The SENT model with the addition of the crack closure and plastic wake effects used an element size of $0.056 \mathrm{~mm}(56 \mu \mathrm{m})$, which was lower than the Irwin plastic zone for $a_{0}=5.08 \mathrm{~mm}$. This element size was able to resolve the plastic deformation at the crack tip in the SENT model. The plastic deformation left in the path of the growing crack (plastic wake) was also resolved by increasing the crack length on an element-by-element scheme during the FCG test simulation. The crack was grown by $1.016 \mathrm{~mm}$ for each crack length configuration as explained in the previous chapter so that the plastic wake was created. The plastic wake produced lower values of the effective $K$ and $R$ parameters (Figure 5-7), with respect to the SENT model with just the crack closure effect (Figure 5-4). The value of the effective stress ratio $R$ was zero for crack length $a_{10}=10.16 \mathrm{~mm}$, this value was smaller than the calculated by the other two SENT models without and with the crack closure effect $\left(a_{8}=\right.$ $11.176 \mathrm{~mm})$.

Figure 5-25 presents the distribution of the plastic wake for several crack lengths analyzed during the FCG simulation of test case 2 with a low stress ratio. 

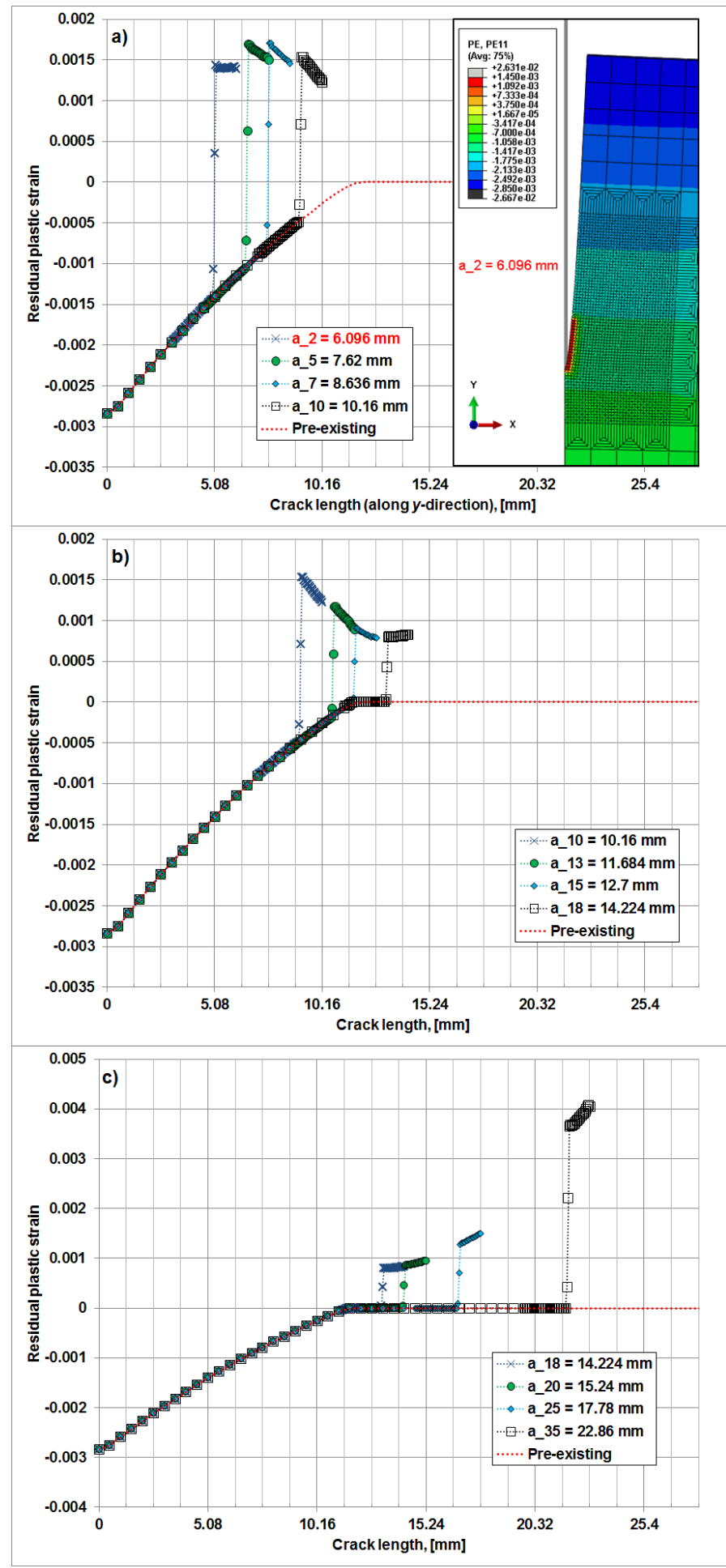

Figure 5-25 Distribution of the plastic wake for specimen C5B3 $\left(P_{\max }=35 \mathrm{kN}\right.$ and $R=$ 0.05) during the FCG simulation. Crack length configurations: a) $a_{2}=6.096 \mathrm{~mm}, a_{5}=$ $7.62 \mathrm{~mm}, a_{7}=8.636 \mathrm{~mm}, a_{10}=10.16 \mathrm{~mm}$; b) $a_{10}=10.16 \mathrm{~mm}, a_{13}=11.684 \mathrm{~mm}, a_{15}=$ 
$12.7 \mathrm{~mm}, a_{18}=14.224 \mathrm{~mm}$; and c) $a_{18}=14.224 \mathrm{~mm}, a_{20}=15.24 \mathrm{~mm}, a_{25}=17.78 \mathrm{~mm}$ and $a_{35}=22.86 \mathrm{~mm}$. The residual plastic strain field produced by the four-point bending simulation is shown for reference (Pre-existing; dotted red line).

In addition, the residual plastic strain in the unnotched-beam model produced by the fourpoint bending simulation is also presented in Figure 5-25 by a dotted line in red color. This residual plastic strain field was already presented in Figure 3-11 for the complete range of $y$-coordinates of the unnotched-beam model. Figure 5-25a presents the crack lengths $a_{2}=6.096 \mathrm{~mm}, a_{5}=7.62 \mathrm{~mm}, a_{7}=8.636 \mathrm{~mm}, a_{10}=10.16 \mathrm{~mm}$, and a contour plot of the residual plastic strain field for crack length $a_{2}=6.096 \mathrm{~mm}$. The crack length was along the $y$-coordinate and the plastic wake was developed behind the crack tip as observed in Figure 5-25a. Figure 5-25b presents the crack lengths $a_{10}=10.16 \mathrm{~mm}, a_{13}=$ $11.684 \mathrm{~mm}, a_{15}=12.7 \mathrm{~mm}$ and $a_{18}=14.224 \mathrm{~mm}$, while Figure $5-25 \mathrm{c}$ presents the crack lengths $a_{18}=14.224 \mathrm{~mm}, a_{20}=15.24 \mathrm{~mm}, a_{25}=17.78 \mathrm{~mm}$ and $a_{35}=22.86 \mathrm{~mm}$.

The plastic wake for crack length $a_{2}=6.096 \mathrm{~mm}$ was constant, i.e. the plastic deformation left in the path of the growing crack presented a constant value of 0.0014 as observed in Figure 5-25a (blue times markers). The plastic wake was shown just for a length of $1.016 \mathrm{~mm}$ behind the crack tip, because the fatigue crack was increased on an element-by-element scheme just for 18 elements. The plastic wake decreased for crack lengths $a_{5}=7.62 \mathrm{~mm}, a_{7}=8.636 \mathrm{~mm}, a_{10}=10.16 \mathrm{~mm}, a_{13}=11.684 \mathrm{~mm}$ and $a_{15}=12.7$ $\mathrm{mm}$. The minimum value of the plastic deformation was 0.0008 for crack length $a_{15}=$ $12.7 \mathrm{~mm}$ (Figure 5-25b). The value of the plastic deformation left in the path of the growing crack increased exponentially for crack lengths from $a_{15}=12.7 \mathrm{~mm}$ to $a_{40}=25$ 
$.4 \mathrm{~mm}$ (Figure 5-25c). This indicated that the size of the plastic zone developed during the FCG test simulation was contained by the initial residual plastic strain produced by the four-point bending process. In other words, the plastic zone decreased during the FCG simulation because of the compressive residual stresses. The yield state of the finite elements for crack lengths $a_{0}=5.08 \mathrm{~mm}, a_{10}=10.16 \mathrm{~mm}, a_{30}=20.32 \mathrm{~mm}$ and $a_{40}=25.4$ $\mathrm{mm}$ in the SENT model with the addition of the crack closure and plastic wake effects is presented in Figure 5-26. The yield state is presented in Figure 5-26 for the maximum applied load $\left(P_{\max }=35 \mathrm{kN}\right)$ and according to the FCG simulation for test case 2 with a low stress ratio.
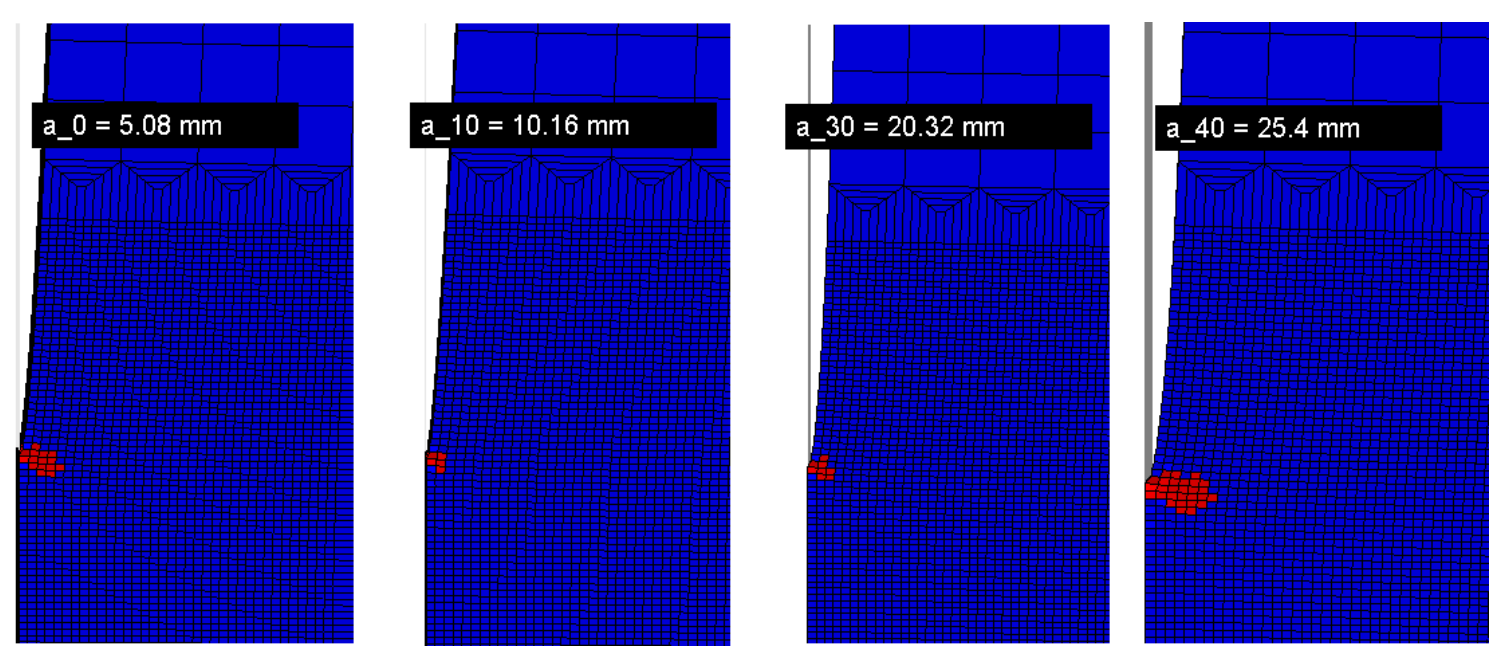

Figure 5-26 Yield state of the finite elements for several crack lengths subjected to $P_{\max }=$ $35 \mathrm{kN}$ (test case 2), according to SENT model with the addition of the crack closure and plastic wake effects.

The red color in Figure 5-26 indicated a plastic state for the element, while the blue color indicated an elastic state. The number of elements with a plastic state was lower for crack 
length $a_{10}=10.16 \mathrm{~mm}$ with respect to the initial crack length $a_{0}=5.08 \mathrm{~mm}$. Then, the number of elements with a plastic state was larger for crack length $a_{30}=20.32 \mathrm{~mm}$ with respect to crack length $a_{10}=10.16 \mathrm{~mm}$. Finally, the crack length $a_{40}=25.4 \mathrm{~mm}$ presented the large number of elements with a plastic state. The plot of the yield state supported the previous statement that the plastic zone size decreased during the FCG simulation because of the compressive residual stresses.

For the negative stress ratio (nominal $R=-1$ ) used in the FCG analysis of test case 3 with a negative stress ratio, a retardation effect was also found in the FCG rate as shown by the experimental measurements (Figure 5-18). This retardation effect was accurately calculated by the SENT model without the crack closure effect, while the other two SENT models presented a poor correlation with respect to the experimental measurements. In this test case, the best estimation of the fatigue life was also done by the SENT model without the crack closure effect, which showed a good correlation with respect to the experimental $a$ vs $N$ curve.

\subsection{Discussion}

The results of the experimental study and computer simulations demonstrate several important features that should be taken into account when analyzing the FCG in the materials with RSF. 
First:

The redistribution of residual stresses resulting from the crack propagation can play an important role under the conditions which lead to the development of different modes of the crack growth (i.e., with and without crack closure) during the FCG process. For example, in the experiments and computer models of the FCG under the load cycles with the nominal stress ratio $R=0.05$ the deceleration of the crack growth rate occurs at the crack length of $\sim 10.5 \mathrm{~mm}$ (Figure 5-15). This length coincides with the length at which the redistributed RSF at the crack tip changes from tensile to compressive and the crack closure becomes possible. The original RSF in the unnotched specimen changes from tensile to compressive at the depth of $5.5 \mathrm{~mm}$ from the surface of the specimen. Therefore, the change in the growth rate (the retardation) is produced at the crack length determined by the redistributed RSF and not by original RSF. If the original RSF was used within a simple superposition model, then the crack growth retardation will be starting at the crack length of $5.5 \mathrm{~mm}$ (Figure 5.14 a). Such a FCG model would underestimate the growth rate and produce less conservative results.

It is necessary to note that if the first RSF region through which the crack propagates is compressive, then the stress redistribution will delay the transition from compressive to tensile stresses and enhance the level of compressive stresses. In such situation, typical for the effect of a cold hole expansion or shot peening treatments on the FCG originating from the treated surface, the omission of stress redistribution effects will result in the overestimation of the crack growth rate and produce more conservative estimates. 
In the case of the FCG test and simulations with the nominal $R=-1.0$ the crack closure occurs at all crack lengths with the crack tip in both tensile and compressive RSF regions. In this case, the deceleration of the crack growth starts when the crack tip is still in the tensile redistributed RSF region (Figure 5-19), although as the crack propagates into the compressive region of the redistributed RSF, the growth rate continues to decrease (Figure 5-19) as the effective stress ratio decreases (Figure 5-3 b). At the length of $\sim 12 \mathrm{~mm}$ the effect of the increase in the crack length on the effective $K_{\max }$ (Figure 5-3 a) becomes more important than the reduction in the effective $R$ and the crack growth starts to accelerate with the increase in $K_{\max }$.

In the case of the FCG test and simulations with the nominal $R=0.7$, the crack closure does not occur at any crack length and the crack growth rate increases monotonously. In this case, in the absence of crack closure effects, a simple model without RSF redistribution effects should produce a good agreement with the experiment.

\section{Second:}

The comparison of the results of the FCG experiments with the simulation results indicates that under different nominal $R$ values different types of simulation models produce a better agreement with the experimental data. This difference can be related to the importance of the crack closure effects and how they are accounted for in the effective $\Delta K$ calculation, and to the accuracy of the Harter T-method at different $R$ values.

In the tests and simulations with the nominal $R=0.05$, the model with rigid crack closure conditions (Section 4.6, Figure 4-15) and with a plastic wake effect produces 
crack growth rates which are in the best agreement with the experimental results within stage II of the FCG (Figure 5-14 b). It slightly underestimates the growth rate while two other models (with rigid crack closure conditions but without the plastic wake and without both rigid crack closure conditions and the plastic wake) produce significantly overestimated growth rates. (However, it is necessary to note that such an overestimation of the FCG rate can result in a more conservative FCG life evaluation, which can be more important for practical applications).

When the crack tip reaches the region of the compressive redistributed RSF, the difference between the crack growth rate in the model with the rigid crack closure conditions and with the plastic wake effect and the experimental results slightly increases (Figure 5-15) resulting in larger underestimation of the crack growth rate in the model, even though this model still remains the closest to the experimental data as compared to other models. This effect can be related to the effective $\Delta K$ calculation method using rigid crack closure conditions. In this approach the minimum value of the effective stress intensity, $K_{\min }$, is limited by the zero value, and it is considered that in the FCG the negative part of the stress cycle does not produce any contribution into the driving force for the FCG. Such an approximation is still commonly used in practice [95] and presented in many textbooks; however, it has been shown that it can lead to the underestimation of the FCG rate [59]. The negative part of the load cycle produces some contribution in the FCG driving force and increases the overall FCG rate, though not as significantly as the positive part. Unfortunately, there are no well-established models quantitatively describing the effect of the negative part of the load cycle on the FCG. 
The negative effect of the omission of the contribution from the negative part of the stress cycle from the FCG driving force becomes more pronounced in the case of the simulation of the FCG under the cyclic load with the nominal $R=-1.0$. In this case, the model without both the rigid crack closure conditions and the plastic wake produces FCG rates closest to the experimentally observed (Figure 5-18). The introduction of the rigid crack closure conditions results in a significant underestimation of the FCG rate. When the rigid crack closure conditions are used together with the plastic wake, the difference between the experimental and computational results becomes even worse. These effects are produced because the introduction of the rigid crack closure conditions results in the elimination of the part of the cycle with a negative effective stress intensity from the FCG driving force. The addition of the plastic wake effect shifts the crack closure towards higher effective $K$ values and, thus, further reduces the FCG driving force in the computational model. Therefore, for the modeling of the FCG with large negative effective $R$ values (resulting from either the applied load or the RSF) it can be important to develop an FCG model which has a more accurate representation of the negative stress effect on the FCG rate.

In [59] it was suggested that when a full crack closure is achieved, the rigid crack closure condition should be removed. A simple superposition was used in this case in [59]. In the present study, a model with the RSF redistribution and effective $K$ calculation without the rigid crack closure conditions was used (the corresponding curves are shown in Figure 5-18 and Figure 5-21). In both cases, a good agreement with the experiment was obtained; however, such an ad-hoc solution does not have any theoretical substantiation and the limits of its applicatiblity are not known. A better understanding of 
the role of the negative part of the load cycle in the FCG and knowledge of the physical mechanisms involved in it are necessary for the development of more physics-based FCG models for such conditions.

In the case of the FCG with the nominal $R=0.7$, the introduction of the rigid crack closure condition does not produce any changes in the FCG process because the crack closure does not occur at all in any RSF regions. The introduction of the plastic wake affects the FCG, however, in this case it has a different mechanism as compared to the FCG with the nominal $R=0.05$ and $R=-1.0$. When $R=0.05$ or $R=-1.0$, the main effect produced by the plastic wake is a shift of the crack closure towards higher applied stress values and the extention of the part of load cycle in which the effective $\Delta K$ is equal to zero under the rigid crack closure condition. In the FCG with nominal $R=0.7$, the plastic wake does not result in the crack closure which is not produced in this case at any crack length and in any RSF regions whether plastic wake is used or not. Therefore, the plastic wake effect on the FCG rate can be the result of only the additional stress redistribution associated with it and the corresponding changes in the effective $K$ values. The mesh resolution required for an accurate simulation of these effects can be significantly higher than that sufficient for capturing of the enhanced crack closure effects occurring at the lower nominal $R$ values, which may serve as a reason for the worst performance of the model with the plastic wake in the simulations with the nominal $R=0.7$.

It should be noted that in the model with baseline FCG curve at $R=0.7$ the RSF redistribution plays no noticeable role in the FCG, as illustrated in Figure 5-1a with $\Delta K$ presented as a function of crack length (the straight line). That is, the contributions from the RSF into $K_{\max }$ and $K_{\min }$ are cancelled in $\Delta K$ and, therefore, the redistribution of the 
RSF and the changes of locations wherein the transition from tensile to compressive conditions (or from compressive to tensile conditions) at the crack tip produced during the FCG are not affecting the crack growth rate. Therefore, a simple superposition model may be sufficient in this case.

Table 5-4 presents a summary of the parameters that should be considered in order to model the FCG with different stress ratios in the components with a variable RSF.

Table 5-4 Summary of parameters required to model the FCG in components with a variable RSF.

\begin{tabular}{|c|c|c|c|c|}
\hline \multirow[b]{2}{*}{ Stress ratio, $R$} & \multicolumn{3}{|c|}{ Parameters } & \multirow{2}{*}{$\begin{array}{l}\text { Correlation Scheme } \\
\qquad \frac{d a}{d N}=f(\Delta K, R)\end{array}$} \\
\hline & $\begin{array}{c}\text { Redistribution } \\
\text { of RSF }\end{array}$ & closure & Plastic Wake & \\
\hline 0.7 & Not important & Not $\mathrm{i}$ & No & Baseline FCG curve \\
\hline 0.05 & Yes & Yes & Yes & Harter \\
\hline-1 & Yes & No & No & Harter T-method \\
\hline
\end{tabular}




\section{Chapter: Conclusions}

A well-defined, consistent and repeatable residual stress field was generated by a four-point bending simulation of an unnotched beam specimen of the 7050-T7451 aluminum alloy. The simulation was conducted with the commercially-available finite element program ABAQUS ${ }^{\mathrm{TM}}$, which used an elastic-plastic model with the combined hardening rule. The distribution of the RSF along the symmetry plane of the FEA model and specimens used in the experiments presented a zigzag pattern. This pattern is characterized by three neutral points of the zero magnitude and two alternating regions with tension and compression distribution. The peak points of the alternating regions were $+90,-60,+65$ and $-110 \mathrm{MPa}$ according to the FEA model. A good agreement (the difference was lower than 12\%) was found between the FEA results and the experimental measurements with the slitting method.

The definition of the yield strength used in ABAQUS ${ }^{\mathrm{TM}}$ was crucial for the accurate determination of the RSF. The standard definition with the $0.2 \%$ proof stress overestimated the RSF produced by the four-point bending by more than $30 \%$. The peak points in the RSF distribution were $+120,-85,+85$ and $-150 \mathrm{MPa}$, according to the FEA model with the yield strength defined at the $0.2 \%$ proof stress. The FEA model determined an accurate RSF when the yield strength was defined with the $0.025 \%$ proof stress.

A FCG model was developed based on the effective stress-intensity factor $K$, wherein the redistributed RSF (because of the FCG) were used to compute effective stress-intensity factors at the maximum and minimum applied cyclic loads. The FCG 
model used the finite element method and the displacement correlation technique to compute the effective $K$. A mesh convergence analysis demonstrated that the computation of the effective $K$ was independent of the element size employed in the finite element model.

The residual stresses produced by the four-point bending were redistributed during the FCG process. Tensile residual stresses in the SENT model increased their magnitude during the redistribution, and the compressive residual stresses in the 5.588 $\mathrm{mm}$ to $10.16 \mathrm{~mm}$ range of $y$-coordinates in the SENT model were redistributed into tensile.

The experimental tests with constant amplitude cyclic loading were performed to obtain the baseline FCG curves ( $d a / d N$ vs. $\Delta K$ ) for the 7050-T7451 aluminum alloy for the stress ratio $R$ of $0.7,0.05$ and -1 . The Harter T-method was used to generate additional baseline $d a / d N$ vs. $\Delta K$ curves for any stress ratio of interest. The Harter Tmethod in conjunction with the effective parameters $\Delta K$ and $R$ were used in order to predict the FCG rate in RSF.

The FCG model was able to predict the retardation effect in the FCG rate because of the compressive RSF. In addition, the transient behavior (increase and decrease) of the FCG rate because of the variable RSF (tension-compression regions) was also captured by the FCG model.

For the FCG test scenario with a large stress ratio $(R=0.7)$, the combination of the SENT model without the crack closure effect and the baseline FCG curve at $R=0.7$ (numerical baseline $R=0.7$ ) produced the best results of the FCG rate for the SENT specimen of the 7050-T7451 aluminum alloy. It was shown that compressive residual 
stresses in front of the crack were eliminated by the minimum applied load in the SENT model. Thus, no retardation effect was observed because of the compressive RSF, and the stress ratio $R$ was not affected by the RSF. In addition, the finite element model demonstrated that the crack was open during the FCG process in spite of the compressive residual stresses so that the crack closure effect was not present.

For the FCG test scenario with a low stress ratio $(R=0.05)$, the SENT model with the addition of the crack closure and plastic wake effects produced the best results of the FCG rate for the SENT specimen of 7050-T7451 aluminum alloy. The finite element results demonstrated that the crack was closed at the minimum applied load during the FCG simulation when the RSF was in compression. In addition, the transition from a tensile to a compressive distribution in the RSF produced a retardation effect in the FCG rate, which was accompanied by a decrease in the plastic zone size at the crack tip and the plastic wake behind the crack tip. An experimental test conducted in order to verify the results of the FCG model demonstrated that the SENT model with the addition of the crack closure and plastic wake effects over-estimated the fatigue life of the SENT specimen by 1.26 when the calculated $d a / d N$ is divided over the measured $d a / d N$.

For the FCG test scenario with a negative stress ratio $(R=-1)$, the SENT model without the crack closure effect produced the best results of the FCG rate and the fatigue life for the SENT specimen of the 7050-T7451 aluminum alloy. The FCG model demonstrated that negative values of the effective $K_{\min }$ were required for an accurate calculation of the FCG rate. Alternatively, the addition of the crack closure effect to the SENT model prevented the appearance of the negative values of the effective $K_{\min }$, which resulted in the underestimation of the FCG rate. The experimental test conducted to 
verify the results of the FCG model demonstrated that the effective $K$ without the crack closure and plastic wake effects over-estimated the fatigue life of the SENT specimen by 1.08 when the calculated $d a / d N$ is divided over the measured $d a / d N$. 


\section{Chapter: Future work}

- Modelling of two-dimensional and three-dimensional crack length configurations

The current manuscript was focused on the analysis of a SENT model with a through-thickness crack, where the initial crack front was assumed to be onedimensional. However, the numerical model can be used to analyze corner crack configurations where the crack front is two-dimensional from the very beginning of FCG and cannot be idealized as one-dimensional. For instance, a corner crack in cold-hole expanded specimens is a possible configuration that can be analyzed. Further, the numerical model can be extended to analyze three-dimensional crack length problems where the crack path trajectory diverges from a straight shape. Crack kinking and turning theories can be included into the numerical model for the three-dimensional analysis of FCG.

\section{- Variable-amplitude cyclic loading}

The finite element model of the SENT specimen with the incorporation of the crack closure and plastic wake effects was able to resolve the evolution of the plastic zone during the FCG process. Mechanical components subjected to high cycle fatigue applications experiences variable loads enclosed to the elastic range of the base material, but it is also common that the mechanical components experience overloads in service. These overloads induce compressive RSF at the crack tip which delay the FCG rate. This problem can be analyzed by the finite 
element model with the incorporation of the crack closure and plastic wake effects.

- Threshold and final fracture regions of the $d a / d N$ vs. $\Delta K$ curve

The numerical model developed to analyze the FCG in RSF is suitable for large cracks in the linear region of the $d a / d N$ vs. $\Delta K$ curve. The extension of the numerical model to analyze the threshold and the final fracture regions of the $d a / d N$ vs. $\Delta K$ curves is another possible future research direction. 


\section{References}

[1] R. P. Reed, J. H. Smith and B. W. Christ, "The economic effects of fracture in the United States, Vol. 647-1," U.S. Department of Commerce, Gaitherburg, Md, USA, 1983.

[2] S. Suresh, Fatigue of Materials, Cambridge, U. K.: Cambridge University Press, 1998.

[3] R. J. H. Wanhill, "Milestone case histories in aircraft structural integrity," National Aerospace Laboratory NLR, 2002.

[4] R. McClung, "A literature survey on the stability and significance of residual stresses during fatigue," Fatigue \& Fracture of Engineering Materials \& Structures, vol. 30, pp. 173-205, 2006.

[5] A. T. Ozdemir and L. Edwards, "Relaxation of residual stresses at cold-worked fastener holes due to fatigue loading," Fatigue \& Fracture of Engineering Materials \& Structures, vol. 20, pp. 1443-1451, 1997.

[6] M. E. Fitzpatrick and L. Edwards, "Fatigue crack/residual stress field interactions and their implications for damage-tolerant design," Journal of Materials Engineering and Performance, vol. 7, no. 2, pp. 190-198, 1998.

[7] Y. C. Lam and K. S. Lian, "The effect of residual stress and its redistribution on fatigue crack growth," Theoretical and Applied Fracture Mechanics, vol. 12, pp. 59-66, 1989.

[8] J. E. LaRue and S. R. Daniewicz, "Predicting the effect of residual stresses on 
fatigue crack growth," International Journal of Fatigue, pp. 508-515, 2007.

[9] X. Yigeng, P. Gregson and I. Sinclair, "Systematic assessment and validation of compliance based crack closure measurements in fatigue," Materials Science and Engineering A, vol. 284, pp. 114-125, 2000.

[10] N. E. Dowling, Mechanical Behavior of Materials, Prentice Hall, 1993.

[11] A. T. Zehnder, Fracture Mechanics, Lecture Notes, New York, 2010.

[12] R. P. Wei, Fracture Mechanics: Integration of Mechanics Materials Science and Chemistry, Cambridge University Press, 2010.

[13] W. Schutz, "A history of fatigue," Engineering Fracture Mechanics, vol. 54, pp. 283-300, 1996.

[14] J. A. Collins, Failure of Materials in Mechanical Design, John Wiley \& Sons, Inc., 1993.

[15] A. J. McEvily, Metal failures: mechanisms, analysis, prevention, John Wiley \& Sons, 2002.

[16] S. Kobayashi, M. Hirata, S. Tsurekawa and T. Watanabe, "Grain boundary engineering for control of fatigue crack propagation in austenitic stainless steel," Engineering Procedia, vol. 10, pp. 112-117, 2011.

[17] T. L. Anderson, Fracture Mechanics: Fundamentals and Applications, CRC Press, 1991.

[18] S. S. Manson and G. R. Halford, Fatigue and Durability of Structural Materials, ASM International, 2006.

[19] A. Butler, "Manufacturing defect at root of F-15 grounding," Aviation Week and 
Space Technology, vol. 168, 2008.

[20] G. E. Dieter and L. C. Schmidt, Engineering Design, 4 ed. ed., McGraw-Hill Higher Education, 2009.

[21] ASTM-E466, Standard practice for conducting force controlled constant amplitude axial fatigue tests of metallic materials, American Society for Testing and Materials, 2007.

[22] G. E. Dieter, Mechanical Metallurgy, McGraw-Hill Book Company, 1986.

[23] S. S. Manson and M. H. Hirschberg, Fatigue: An Interdisciplinary Approach, Syracuse, N.Y.: Syracuse University Press, 1964.

[24] H. Neuber, "Theory of stress concentration for shear-strained prismatical bodies with arbitrary nonlinear stress-strain law," Journal of Applied Mechanics, vol. 28, no. 4, pp. 544-550, 1961.

[25] ASTM-E647, Standard Test Method for Measurement of Fatigue Crack Growth Rates, American Society for Testing and Materials, 2011.

[26] P. J. Withers, "Residual stress and its role in failure," Reports on Progress in Physics, vol. 70, pp. 2211-2264, 2007.

[27] P. Withers and H. Bhadeshia, "Residual Stress Part 1 - Measurements Techniques," Materials Science and Technology, vol. 17, pp. 335-365, $2001 \mathrm{a}$.

[28] R. A. Winholtz, "Residual stresses: Macro and micro stresses," in Encyclopedia of Material: Science and Technology, B. e. al., Ed., Elsevier, 2001, pp. 8148-8152.

[29] P. J. Withers and H. K. D. H. Bhadeshia, "Residual stress: II Nature and origins," Materials Science and Technology, pp. 366-375, $2001 \mathrm{~b}$. 
[30] M. F. Ashby and D. R. H. Jones, Engineering Materials - An introduction to their properties and applications, Oxford-Pergamon, 1980.

[31] E. J. Hearn, Mechanics of materials, vol. 2, Elsevier, 1997.

[32] A. P. Parker, "Residual stress effects in fatigue," ASTM STP, vol. 776, pp. 13-31, 1982.

[33] G. Glinka, "Effect of residual stresses on fatigue crack growth in steel weldments under constant and variable loads," ASTM-STP, vol. 677, pp. 198-214, 1979.

[34] N. E. Frost, K. J. Marsh and L. P. Pook, Metal Fatigue, Clarendon Press, Oxford, 1974.

[35] M. Beghini and L. Bertini, "Fatigue crack propagation through residual stress fields with closure phenomena," Engineering Fracture Mechanics, vol. 36, no. 3, pp. 379-387, 1990.

[36] L. Bertini, "A quantitative assessment of the residual stress effects on fatigue crack propagation in air and seawater," Proc. 2nd Int. Conf. on Fatigue and Stress of Engineering Materials and Components, 1988.

[37] W. Elber, "Fatigue crack closure under cyclic tension," Engineering Fracture Mechanics, vol. 2, pp. 37-44, 1970.

[38] M. N. James and J. F. Knott, "An assesment of crack closure and the extent of the short crack regimen in Q1N (HY 80) steel," Fatigue of Engineering Materials and Structures, vol. 8, pp. 177-191, 1985.

[39] S. Suresh and R. O. Ritchie, "Propagation of short fatigue cracks," International Metallurgical Reviews, vol. 29, pp. 445-476, 1984. 
[40] R. K. T. Venkateswara, W. Yu and R. O. Ritchie, "Fatigue crack propagation in aluminum-lithium alloy 2090: Part I. Long crack behavior," Metallurgical Transactions, vol. 19A, pp. 549-561, 1988.

[41] D. A. Porter and K. E. Easterling, Phase transformations in metals and alloys, London, UK.: Chapman \& Hall, 1992.

[42] J. Schijve, "Fatigue crack closure: Observations and technical significance," ASTM STP, vol. 982, pp. 5-34, 1988.

[43] R. C. McClung, "On the finite element analysis of fatigue crack closure - 1. Basic modeling issues," Engineering Fracture Mechanics, vol. 33, pp. 237-252, 1989.

[44] R. C. McClung, "Finite element analysis of fatigue crack closure: a historical and critical review," Fatigue 99: The seventh international conference, 1999.

[45] K. Solanki, S. R. Daniewicz and J. C. Newman Jr., "Finite element analysis of plasticity-induced fatigue crack closure: an overview," Engineering Fracture Mechanics, vol. 71, pp. 149-171, 2004.

[46] V. Lacarac, A. A. Garcia-Granada, D. J. Smith and M. J. Pavier, "Prediction of the growth rate for fatigue cracks emanating from cold expanded holes," International Journal of Fatigue, vol. 26, pp. 585-595, 2004.

[47] S. Y. Lee, Y. Sun, K. An, H. Choo, C. R. Hubbard and P. K. Liaw, "Evolution of residual-strain distribution through an overload-induced retardation period during fatigue-crack growth," Journal of Applied Physics, vol. 107, 2010.

[48] N. A. Fleck, I. F. C. Smith and R. A. Smith, "Closure behaviour of surface cracks," Fatigue of Engineering Materials and Structures, vol. 6, no. 3, pp. 225-239, 1983. 
[49] LexTech, Inc, "Fracture mechanics and fatigue crack growth analysis software tool (AFGROW)," 2014. [Online]. Available: http://www.afgrow.net/. [Accessed September 2014].

[50] SwRI, "Southwest Research Institute NASGRO," 2014. [Online]. Available: http://www.swri.org/4org/d18/mateng/matint/nasgro/default.htm. [Accessed September 2014].

[51] J. A. Harter, Afgrow users guide and technical manual, AFRL-VA-WP-TR-19993016, 1999.

[52] S. R. Mettu, V. Shivakumar, J. M. Beek, F. Yeh, L. C. Williams, R. G. Forman, J. J. McMahon and J. C. Newman-Jr., "NASGRO 3.0 - A software for analyzing aging aircraft," in NASA Conference Publication, 208982. Aging aircraft, 1999.

[53] R. G. Forman and S. R. Mettu, "Behaviour of surface and corner cracks subjected to tensile and bending loads in Ti-6Al-4V alloy," Fracture Mechanics: 22nd Symposium, Vol. 1, ASTM STP 1131, pp. 519-546, 1992.

[54] K. Walker, "The effect of stress ratio during crack propagation and fatigue for 2024-T3 and 7075-T6 aluminum," Effects of Environment and Complex Load History on Fatigue Life, ASTM STP, vol. 462, pp. 1-14, 1970.

[55] D. V. Nelson, "Effects of residual stress on fatigue crack propagation," ASTM STP, vol. 776, pp. 172-194, 1982.

[56] K. W. Jones and M. L. Dunn, "Predicting corner crack fatigue propagation from cold worked holes," Engineering Fracture Mechanics, vol. 76, pp. 2074-2090, 2009. 
[57] A. Todoroki and H. Kobayashi, "Prediction of fatigue crack growth rate in residual stress fields," Key Engineering Materials, Vols. 51-52, pp. 367-372, 1991.

[58] K. J. Kang, J. H. Song and Y. Y. Earmme, "Fatigue crack growth and closure behaviour through a compressive residual stress field," Fatigue \& Fracture of Engineering Materials \& Structures, vol. 13, pp. 1-13, 1990.

[59] K. W. Jones and M. L. Dunn, "Fatigue crack growth through a residual stress field introduced by plastic beam bending," Fatigue \& Fracture of Engineering Materials \& Structures, pp. 863-875, 2008.

[60] L. R. Hall, R. C. Shah and W. L. Engstrom, "Fracture and fatigue crack growth behavior of surface flaws and flaws originating at fastener holes V1 results and discussion," Technical report AFFDL-TR - Air Force Flight Dynamics Laboratory, 1974.

[61] T. M. Hsu and W. M. McGee, "Extended study of flaw growth at fastener holes," Techical report AFFDL-TR-77-83, 1977.

[62] W. H. Cathey and A. F. Grandt-Jr., "Fracture mechanics consideration of residual stresses introduced by coldworking fastener holes," Journal of Engineering Materials and Technology, vol. 102, no. 1, pp. 85-91, 1980.

[63] G. Clark, "Modeling residual stresses and fatigue crack growth at cold-expanded fastener holes," Fatigue \& Fracture of Engineering Materials \& Structures, vol. 14, no. 5, pp. 579-589, 1991.

[64] Z. Wang and X. Zhang, "Predicting fatigue crack growth life for cold-worked holes based on existing closed-form residual stress models," International Journal of 
Fatigue, vol. 25, pp. 1285-1291, 2003.

[65] M. Kokaly, J. Ransom, B. Jude, H. Restis and L. Reid, "Observations and analysis of fatigue crack growth from cold worked hole," in Proceedings of the 8th Joint FAA/DoD/NASA aging aircraft conference, Palm Springs, CA, 2005.

[66] D. H. Stuart, M. R. Hill and J. C. Newman-Jr, "Correlation of one-dimensional fatigue crack growth at cold-expanded holes using linear fracture mechanics and superposition," Engineering Fracture Mechanics, vol. 78, pp. 1389-1406, 2011.

[67] A. T. DeWald and M. R. Hill, "Eigenstrain-based model for prediction of laser peening residual stresses in arbitrary three-dimensional bodies. Part 1: model description," The Journal of Strain Analysis for Engineering Design, vol. 44, no. 1, pp. 1-11, 2009.

[68] T. Lotz, Development of a consistent experimental method to study residual stress effects on fatigue crack growth, Delft, Netherlands: Delft University of Technology, 2014.

[69] Alcoa, "Alcoa Mill Products," 2014. [Online]. Available: https://www.alcoa.com/mill_products/catalog/pdf/alloy7050techsheetrev.pdf. [Accessed 2014].

[70] D. A. Clarck and W. S. Johnson, "Temperature effects on fatigue performance of cold expanded holes in 7050-T7451 aluminum alloy," International Journal of Fatigue, vol. 25, pp. 159-165, 2003.

[71] J. C. Benedyk, "International temper designation systems for wrougth aluminum alloys: Part II - Thermally treated (T Temper) aluminum alloys," Light Metal Age, 
pp. 16-22, 2010.

[72] ASM Handbook, "Properties of Wrought Aluminum and Aluminum Alloys," in ASM Handbook. Properties and Selection: Nonferrous Alloys and Special Purpose Materials, vol. 2, ASM International, 1990, pp. 62-122.

[73] ASTM-E8, Standard Test Methods for Tension Testing of Metallic Materials, American Society for Testing and Materials, ASTM Int., 2011.

[74] ASTM-E9, Standard Test Method of Compression Testing of Metallic Materials at Room Temperature, American Society for Tensting and Materials, ASTM Int., 2009.

[75] M. B. Prime and M. R. Hill, "Residual stress, stress relief, and inhomogeneity in aluminum plate," Los Alamos National Laboratory, California, U.S., 2002.

[76] E. P. Popov, Engineering Mechanics of Solids, Prentice-Hall, Inc., 1990.

[77] M. A. Sutton, W. J. Wolters, W. H. Peters, W. F. Ranson and S. R. McNeill, "Determination of displacements using an improved digital correlation method," Image and Vision Computing, vol. 1, no. 3, pp. 133-139, 1983.

[78] P. Cheng, M. A. Sutton, H. W. Schreier and S. R. McNeill, "Full-field speckle pattern image correlation with B-Spline deformation function," Experimental Mechanics, vol. 42, no. 3, pp. 344-352, 2002.

[79] H. A. Bruck, S. R. McNeill, M. A. Sutton and W. H. Peters III, "Digital image correlation using Newton-Raphson method of partial differential correction," Experimental Mechanics, vol. 29, no. 3, pp. 261-267, 1989.

[80] W. Cheng and I. Finnie, "An overview of the crack compliance method for residual 
stress measurement," Proceedings of the Fourth International Conference on Residual Stresses: ICRS-4., pp. 449-558, 1994.

[81] M. B. Prime, "Residual stress measurement by succesive extension of a slot: The crack compliance method," Applied Mechanics Review, vol. 52, no. 2, pp. 75-96, 1999.

[82] M. J. Lee and M. R. Hill, "Effect of strain gage length when determining residual stress by slitting," Journal of Engineering Materials and Technology, vol. 129, no. 1, pp. 375-382, 2007.

[83] J. R. Davis, Tensile Testing, ASM International, 2004.

[84] I. Noyan and J. B. Cohen, Residual Stress: Measurement by diffraction and interpretation, Springer-Verlag, 1987.

[85] A. N. Ezeilo and G. A. Webster, "Neutron diffraction analysis of the residual stress distribution in a bent bar," The Journal of Strain Analysis for Engineering Design, vol. 35, no. 4, pp. 235-246, 2000.

[86] P. F. P. De Matos and D. Nowell, "Analytical and numerical modelling of plasticity-induced crack closure in cold-expanded holes," Fatigue \& Fracture of Engineering Materials \& Structures, vol. 31, pp. 408-503, 2008.

[87] K. W. Jones and M. L. Dunn, "Predicting fatigue crack growth from a preyielded hole," International Journal of Fatigue, vol. 31, pp. 223-230, 2009a.

[88] V. Nigrelli and S. Pasta, "Finite-element simulation of residual stress induced by split-sleeve cold-expansion process of holes," Journal of Materials Processing Technology, vol. 205, pp. 290-296, 2008. 
[89] P. Papanikos and S. A. Meguid, "Elasto-plastic finite-element analysis of the cold expansion of adjacent fastener holes," Journal of Materials Processing Technology, Vols. 92-93, pp. 424-428, 1999.

[90] L. Yongshou, S. Xiaojun, L. Jun and Y. Zhufeng, "Finite element method and experimental investigation on the residual stress fields and fatigue performance of cold expansion hole," Materials and Design, vol. 31, pp. 1208-1215, 2010.

[91] M. Beghini, L. Bertini and E. Vitale, "Fatigue crack growth in residual stress fields: Experimental results and modelling," Fatigue \& Fracture of Engineering Materials \& Structures, vol. 17, pp. 1433-1444, 1994.

[92] J. Ahmad and V. H. A. T. Papaspyropoulos, "Elastic-Plastic Analysis of EdgeNotched Panels subjected to Fixed Grip Loading," Engineering Fracture Mechanics, vol. 38, no. 4/5, pp. 283-294, 1991.

[93] S. Corp., Abaqus Documentation, 2011.

[94] D. R. J. Owen and A. J. Fawkes, Engineering Fracture Mechanics: Numerical Methods and Applications, Swansea, UK: Pineridge Ltd, 1983.

[95] G. Totten, "Fatigue crack propagation," in Advanced Materials \& Processes, 2008, pp. 39-41.

[96] J. Lu, Handbook of measurements of residual stresses, Lilburn, GA: Fairmont Press, 1996.

[97] M. B. Prime, "Cross-sectional mapping o residual stresses by measuring the surface contour after a cut," Transactions of ASME, vol. 123, pp. 162-168, 2001.

[98] P. Paris and F. Erdogan, "A critical analysis of crack propagation laws," Journal of 
Basic Engineering, vol. 85, pp. 528-534, 1963.

[99] H. Nisitani and K. Takao, "Significance of initiation, propagation and closure of microcracks in high cycle fatigue of ductile metals," Engineering Fracture Mechanics, pp. 445-456, 1981.

[100] A. A. Griffith, "The phenomena of rupture and flow in solids," Philosophical Transactions of the Royal Society of London, Series A, Containing Papers of a Mathematical or Physical Character, vol. 221, pp. 163-197, 1920.

[101] E. Orowan, "Fracture and strength of solids," Reports on Progress in Physics, vol. XII, pp. 147-166, 1948.

[102] G. R. Irwin, "Onset of fast crack propagation in high strength steel and aluminum alloys," Sagamore research conference proceedings, pp. 289-305, 1956.

[103] H. M. Weestergaard, "Bearing pressures and cracks," Journal of Applied Mechanics, vol. 6, pp. 49-53, 1939.

[104] G. R. Irwin, "Fracture dynamics," Fracturing of Metals, American Society for Metals, pp. 147-166, 1948.

[105] G. R. Irwin, "Analysis of stresses and strains near the end of a crack traversing a plate," Journal of Applied Mechanics, vol. 24, pp. 361-364, 1957.

[106] I. N. Sneddon, "The distribution of stress in the neighbourhood of a crack in an elastic solid," Proceedings of the Royal Society of London, Series A, Mathematical and Physical Sciences, vol. 27, pp. 229-260, 1946.

[107] M. L. Williams, "On the stress distribution at the base of a stationary crack," Journal of applied mechanics, vol. 24, pp. 109-114, 1957. 
[108] B. L. Karihaloo, Fracture Mechanics and Structural Concrete, Harlow, Essex, UK: Addison Wesley, 1995.

[109] ASTM-E466, Standard practice for conducting force controlled constant amplitude axial fatigue tests of metallic materials, American Society for Testing and Materials, ASTM Int..

[110] A. M. Korsunsky, "Eigenstrain analysis of residual strains and stresses," Journal of Strain Analysis, vol. 44, pp. 29-43, 2008.

[111] K. Gall, H. Sehitoglu and Y. Kadioglu, "Plastic zones and fatigue-crack closure under plain-strain double slip," Metallurgical and Materials Transactions A, vol. 27, pp. 3491-3502, 1996.

[112] A. R. Ingraffea, Engineering Fracture Mechanics Lectures Notes, New York, US: Cornell University, 2011.

[113] J. Underwood and L. P. Pook, "Flaw growth and fracture," ASTM STP, vol. 631, pp. 402-415, 1977.

[114] S. Fukuda, "An experimental study of redistribution of welding residual stress," Transactions of JWRI, vol. 7, p. 67, 1978.

[115] N. Chandawanich and W. N. Sharpe Jr., "An experimental study of fatigue crack growth initiation and growth from coldworked holes," Engineering Fracture Mechanics, vol. 11, pp. 609-620, 1979.

[116] D. Kirk, "Shot peening," Aircraft Engineering and Aerospace Technology, vol. 71, pp. 349-361, 1999.

[117] A. King, A. Steuwer, C. Woodward and P. Withers, "Effects of fatigue and fretting 
on residual stresses introduced by laser shock peening," Materials Science and Engineering A, Vols. 435-436, pp. 12-18, 2006.

[118] V. Lacarac and D. J. Smith, "Fatigue crack growth from plain and cold expanded holes in aluminum alloys," International Journal of Fatigue, vol. 22, pp. 189-203, 2000.

[119] USAF, "United States Air Force - Handbook for Damage Tolerant Design," 2014. [Online]. Available: http://www.afgrow.net/applications/DTDHandbook/pdfs.aspx. [Accessed 2014].

[120] P. F. P. de Matos, A. J. McEvily, P. M. G. P. Moreire and P. M. S. T. de-Castro, "Analysis of the effect of cold-working of rivet holes on the fatigue life of an aluminum alloy," International Journal of Fatigue, vol. 29, pp. 575-586, 2007.

[121] G. J. Petrak and R. P. Stewart, "Retardation of cracks emanating from fastener holes," Engineering Fracture Mechanics, vol. 6, pp. 275-282, 1974.

[122] F. Dunne and N. Petrinic, Introduction to Computational Plasticity, Oxford University, Press., 2005.

[123] H. X. B. Pan, K. Qian and A. Asundi, "Two-dimensional digital image correlation for in-plane displacement and strain measurement: a review," Measurement Science and Technology, vol. 20, p. 17pp, 2009.

[124] N. C. Mahendra Babu, T. Jagadish, K. Ramachandra and S. N. Sridhara, "A simplified 3-D finite element simulation of cold expansion of a circular hole to capture through thickess variation of residual stresses," Engineering Failure Analysis, vol. 15, pp. 339-348, 2008. 
[126] D. Löhe, K. -H. Lang and O. Vöhringer, "Residual stresses and fatigue behavior," in Handbook of Residual Stress and Deformation of Steel, G. Totten, M. Howes and T. Inoue, Eds., ASM International, 2002, pp. 27-53.

[127] U. Krupp, A. Giertler, M. Söker, H. Fu, B. Dönges, H. -J. Christ, K. Istomin, A. Hüsecken, U. Pietsch, C. -P. Fritzen and W. Ludwig, "Significance and mechanism of the crack initiation process during very high cycle fatigue of duplex stainless steel," Procedia Engineering, vol. 74, pp. 143-146, 2014.

[128] B. M. Kapadia, "Influence of residual stresses on fatigue crack propagation in electroslag welds," ASTM STP, vol. 648, pp. 244-260, 1978.

[129] S. Pasta, "Fatigue crack propagation from cold-worked hole," Engineering Fracture Mechanics, vol. 74, pp. 1525-1538, 2007.

[130] R. Cook, "Residual stresses induced by cold expansion," Proceedings of the first international conference on computational methods and experimental measurements for surface treatments effects, 1993.

[131] K. W. Jones and M. L. Dunn, "Predicting corner crack fatigue propagation from cold worked holes," Engineering Fracture Mechanics, vol. 76, pp. 2074-2090, $2009 b$.

[132] P. M. Toor, "Cracks emanating from precraked coldworked holes," Engineering Fracture Mechanics, vol. 8, no. 2, pp. 391-395, 1976.

[133] J. Schijve, Fatigue of Structures and Materials, 2nd ed., Springer, 2009.

[134] S. S. Carlson, Application of corrections to model and predict fatigue crack growth at cold expanded holes in 2024-T351 aluminum alloys, University of Utah: MS 
Thesis, 2008.

[135] D. Stefanescu, "Measurement and prediction of fatigue crack growth from cold expanded holes part 2: prediction of fatigue crack growth from cold expanded holes," Journal of Strain Analysis, vol. 39, pp. 41-53, 2004. 